\title{
Engineering the indigoidine-synthesising enzyme BpsA for diverse applications in biotechnology
}

\author{
By
}

Alistair Sinclair Brown

A thesis submitted to the Victoria University of Wellington

in fulfilment of the requirements for the degree of

Doctor of Philosophy in Biotechnology

Victoria University of Wellington

(2018) 



\section{Abstract}

Non-ribosomal peptide synthetases (NRPSs) are large, modular enzymes that synthesise bioactive peptides using an assembly line architecture, wherein each module is responsible for the incorporation of a monomer into the growing chain. Present in both fungi and bacteria, NRPSs are responsible for a wide variety of secondary metabolites and bioactive compounds including siderophores, antibiotics, anti-cancer compounds and immunosuppressants. For functionality, NRPSs require the attachment of a phosphopantetheine moiety to their peptidyl carrier protein domains. This reaction is catalysed by a phosphopantetheinyl transferase (PPTase).

The NRPS $\underline{b}$ lue pigment synthetase $\underline{A}$ (BpsA) is unusual in that it is comprised of only a single module. BpsA contains an adenylation domain that recognises and sequentially binds two molecules of L-glutamine, an oxidation domain that is believed to oxidise each glutamine monomer, a peptidyl carrier protein domain that binds the phosphopantetheine moiety, and a thioesterase domain that cyclises each glutamine and releases the final bicyclic product from the enzyme. This final product is a blue pigment called indigoidine, and its synthesis from two molecules of L-glutamine is powered by ATP. Comparatively to other NRPSs BpsA is easy to manipulate and work with both in vitro and in vivo. Here, the ability to easily detect synthesis of indigoidine was utilised to provide a versatile reporter to detect a variety of biochemical activities.

PPTases are essential enzymes that are promising drug targets in the clinically important bacteria Pseudomonas aeruginosa and Mycobacterium tuberculosis. BpsA can be purified in the inactive apo form, which then requires a PPTase to activate it to enable indigoidine synthesis. Here it was shown that mixing BpsA, a PPTase, the enzymatic substrates, and a potential inhibitor enables screening for PPTase inhibition by monitoring the rate or extent of indigoidine synthesis. This method was optimised and used to screen commercial drug libraries against two PPTases, PcpS from P. aeruginosa and PptT from M. tuberculosis. Several novel inhibitors were identified and pilot in vivo studies were performed. M. tuberculosis also possesses a second essential PPTase called TB-AcpS, which has very narrow substrate specificity and cannot post-translationally modify BpsA. In an attempt to widen the substrate specificity a combination of rational 
engineering and directed evolution was employed. These attempts did not yield significant improvements in the ability of TB-AcpS to activate modified BpsA, however they did yield mutants that were more effective substrates for other type I PPTases.

The easily detectable nature of indigoidine also enabled application of BpsA as a reporter for a range of different substrates. Particularly effective was development of a commercially applicable method using BpsA to quantify L-glutamine in a range of conditions, including cell culture media and blood. The assay developed offers several advantages over currently available kits. BpsA was also used to detect and quantify ATP, and this was applied to monitor adenylation reactions. Finally, the ability of BpsA to synthesise indigoidine-like compounds from glutamine analogues was explored. 


\section{Acknowledgments}

Firstly, thank you to my Supervisor Dr David Ackerley. You've played a huge role in shaping me into the scientist I am today. The positive environment you have fostered in the research group is a testament to your ability as a mentor and leader. Also thank you for putting up with me bursting into your office several times a week, while you're busy, to run some dumb idea l've had past you. The minimal eye rolling and positive encouragement when the occasional good idea gets vomited out is massively appreciated.

Thank you to the past and present Ackerley laboratory members that have made my time in the laboratory so enjoyable. Thanks to the original members who have left (or returned) in no particular order: Laura, Janine, Claire, Jeremy, Elsie, Rory, Katherine, Kate, Becky and Mark. Your advice and help was and is always appreciated. I would also like to thank the current members of the laboratory: Abby, Kelsi, Luke, Jasmine, Michelle, Jack and Matt. I'm looking forward to the Vegas reunion in 2024 when we are all going to be doctors and we can have new arguments about who is the most senior.

I'd like to acknowledge and thank the SBS technicians and office staff that help keep the SBS ticking over. Without you guys nothing in the lab would work and none of us would be able to work.

A huge thank you to those who have provided emotional support throughout this thesis. Thank you to Varun and Luke, for the memes and the friendships. A huge thank you to my other long-suffering friends outside of science that have slowly tapered off the "when are you going to finish?" questions as I got closer to handing in.

Finally, a massive thanks to my parents Colin and Sue, without your emotional and financial support I wouldn't have been able to produce this body of work. You've been the absolute rocks in my life and I'm extremely lucky to have you both. 


\section{Table of contents}

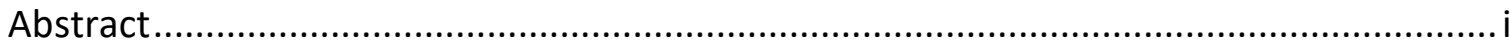

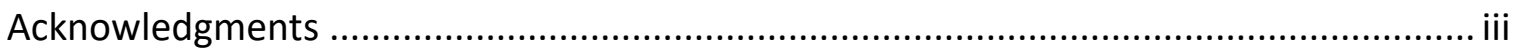

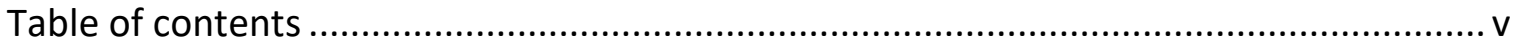

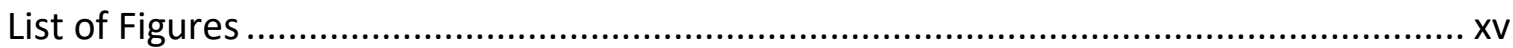

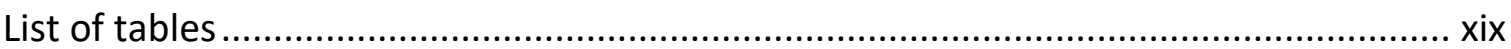

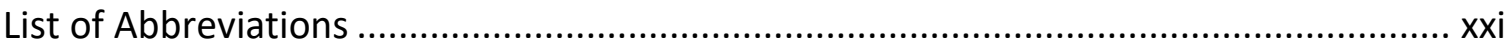

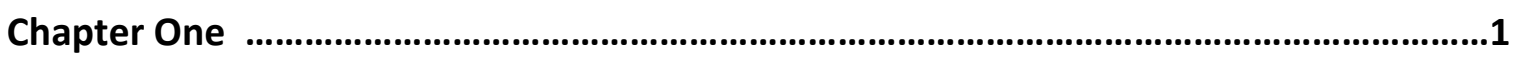

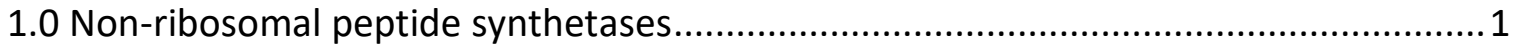

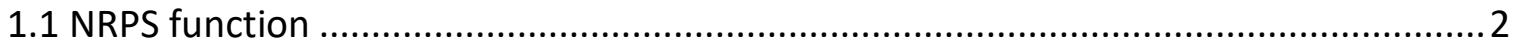

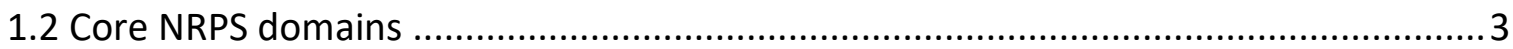

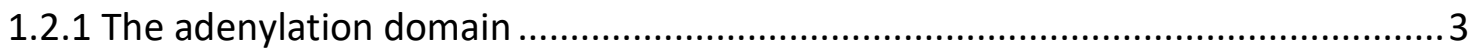

1.2.2 The peptidyl carrier protein domain ............................................................ 4

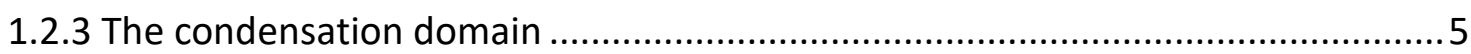

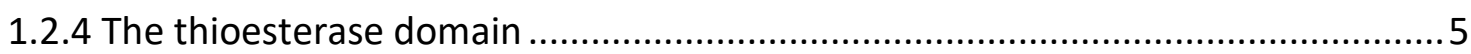

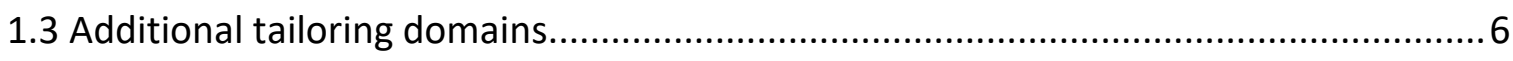

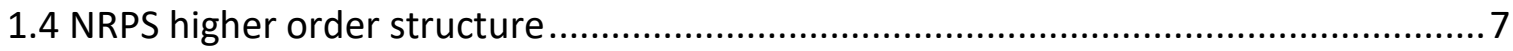

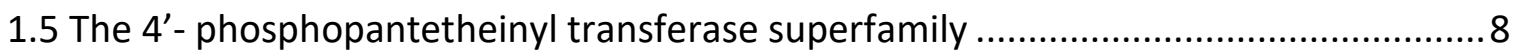

1.5.1 PPTases post-translationally modify carrier proteins..................................... 8

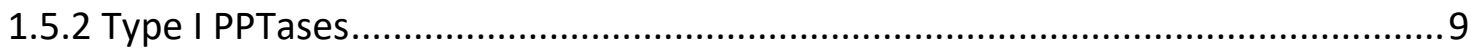

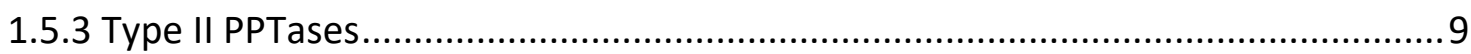

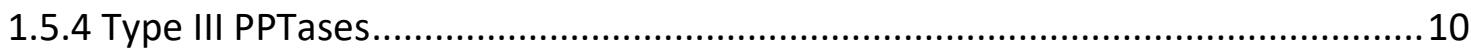

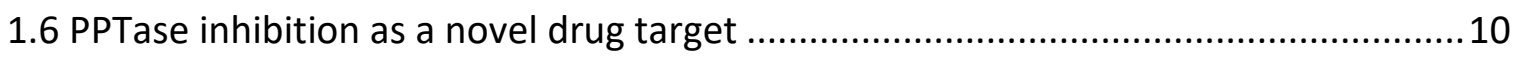

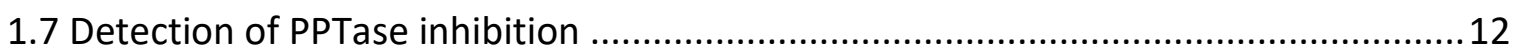

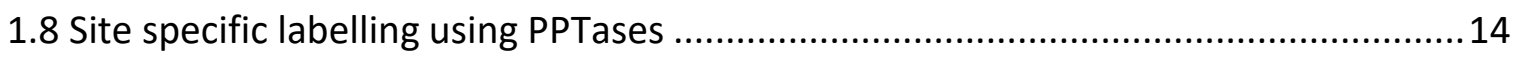


1.9 Utility of PPTases for identifying biosynthetic gene clusters..................................16

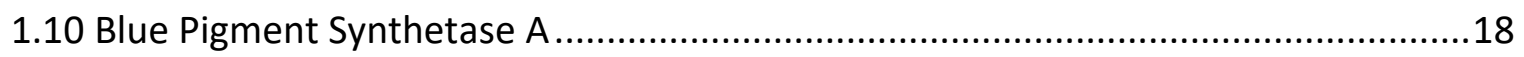

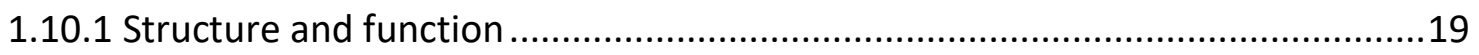

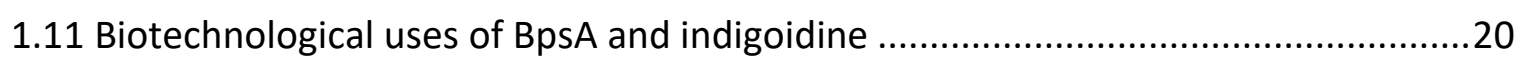

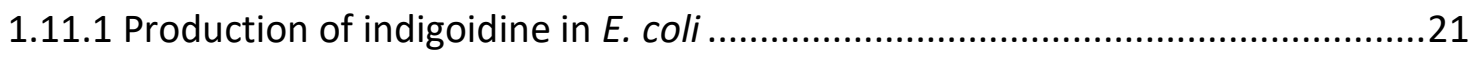

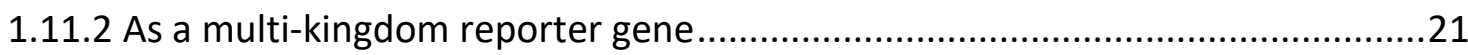

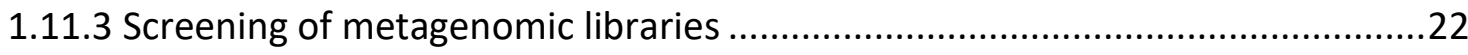

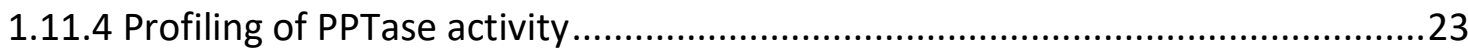

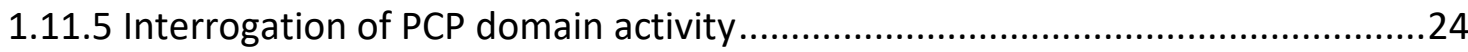

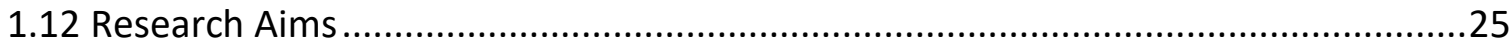

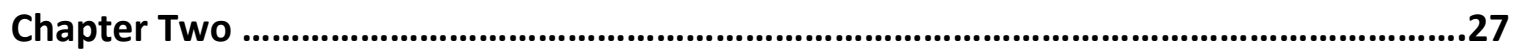

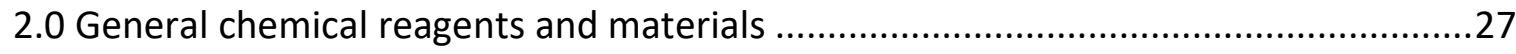

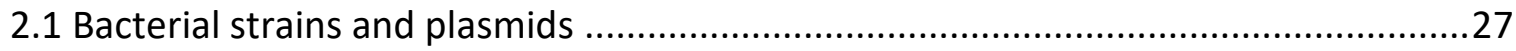

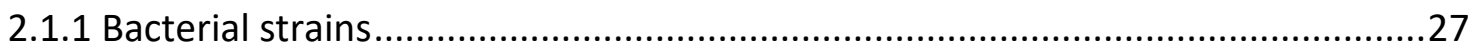

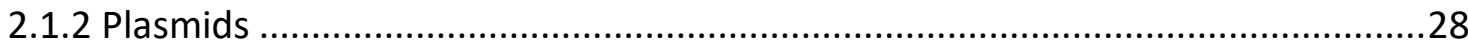

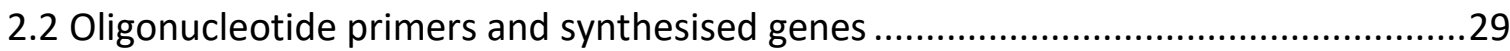

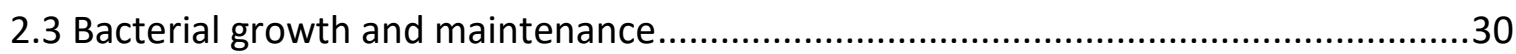

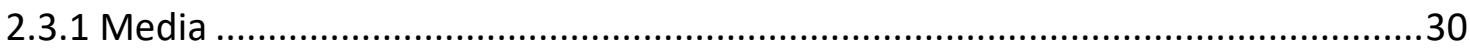

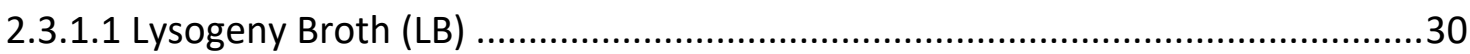

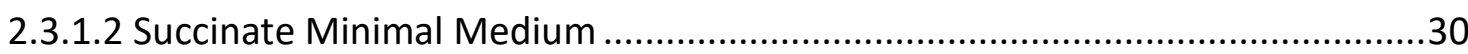

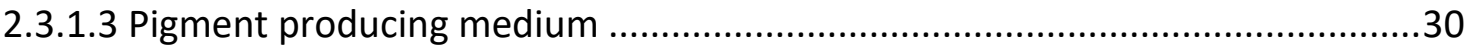

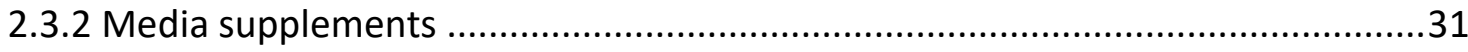

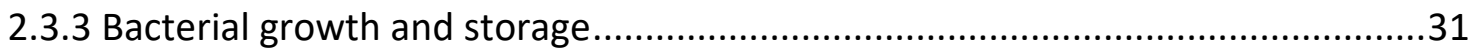

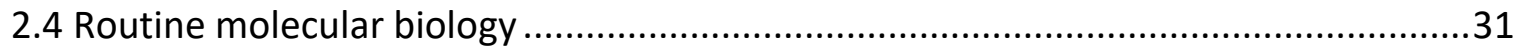

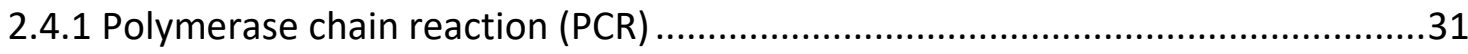

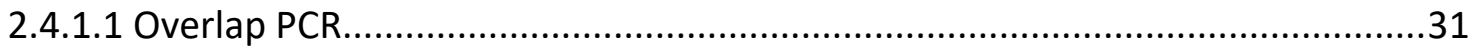




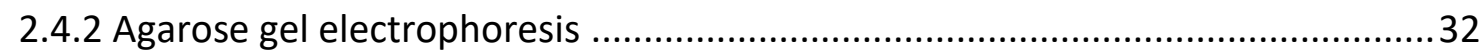

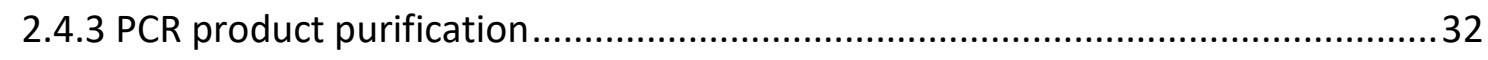

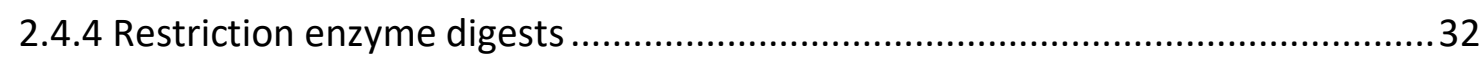

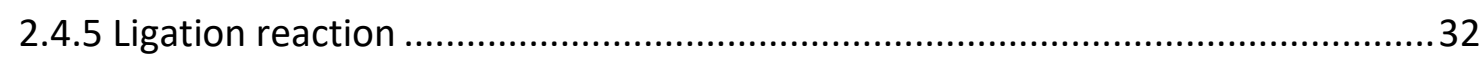

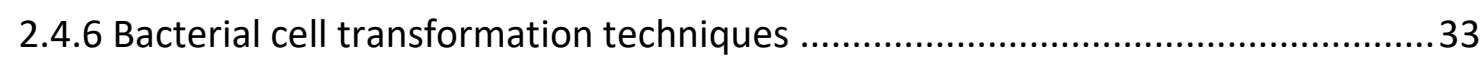

2.4.6.1 Preparation of chemically competent cells ...............................................33

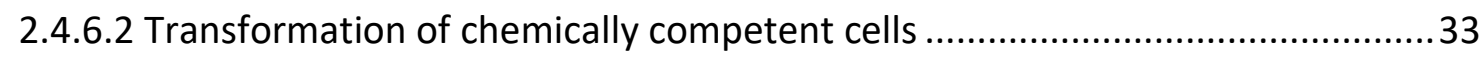

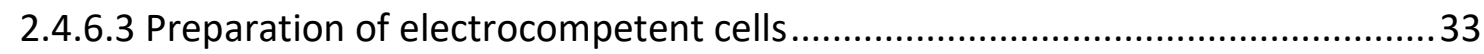

2.4.6.4 Transformation of electrocompetent cells ................................................. 34

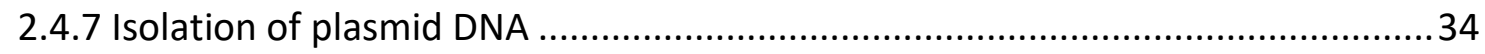

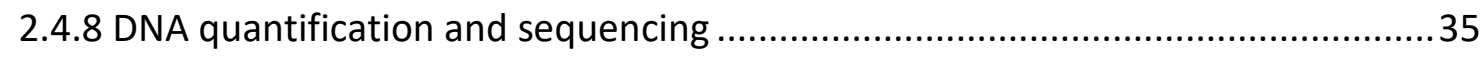

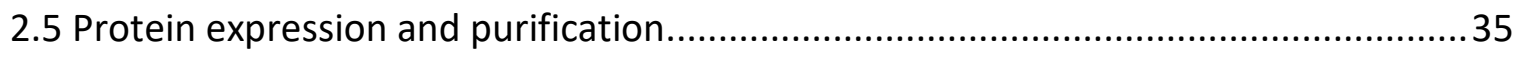

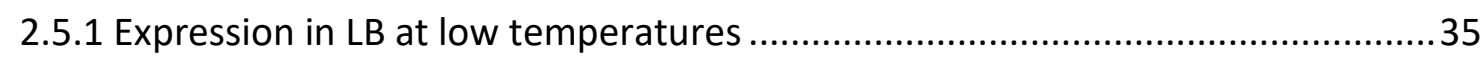

2.5.2 Expression in LB containing betaine and sorbitol at low temperatures ..............35

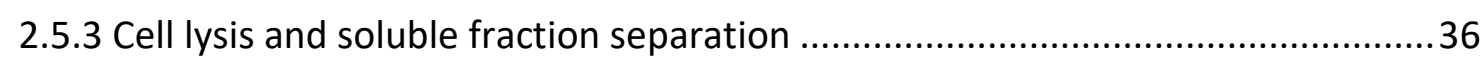

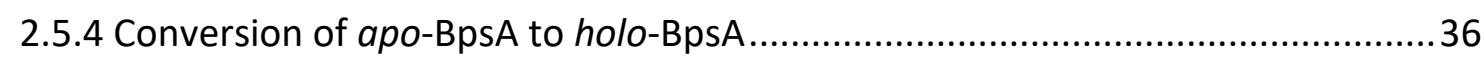

2.5.5 Protein purification via Ni-NTA affinity chromatography .................................36

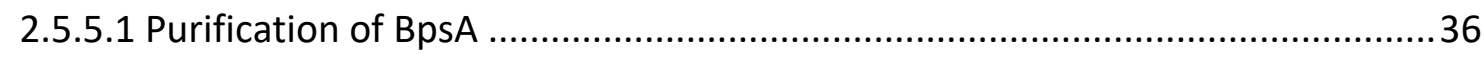

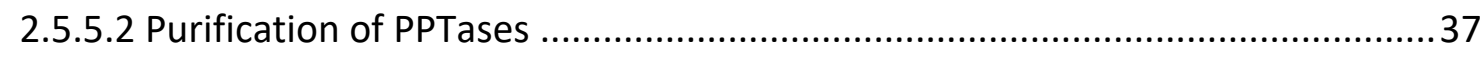

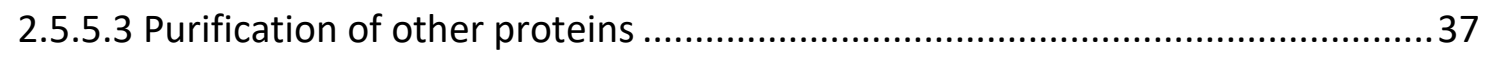

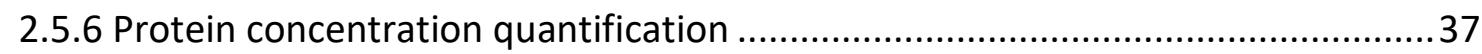

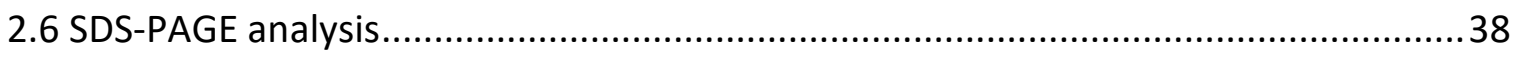

2.7 In vitro assays for the detection of PPTase inhibition ........................................40

2.7.1 PPTase mediated conversion of apo-BpsA to holo-BpsA ................................40

2.7.2 Optimised inhibitor screening protocol..................................................... 40

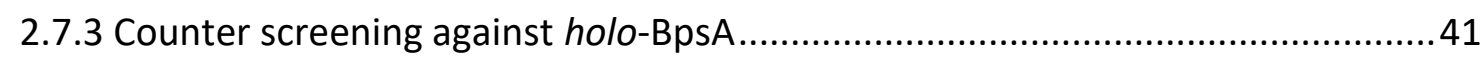




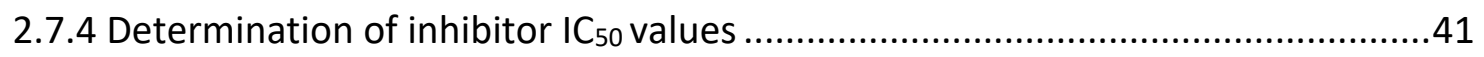

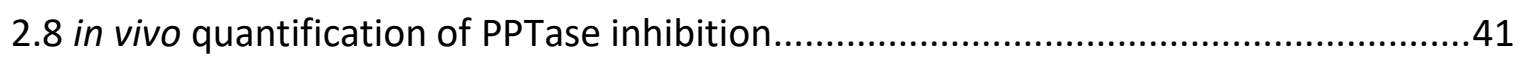

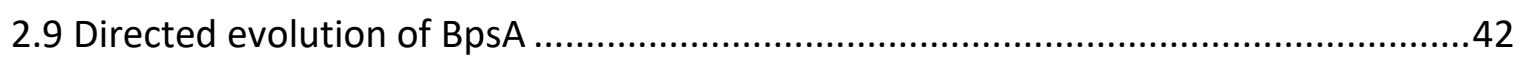

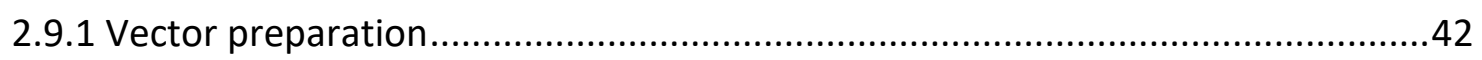

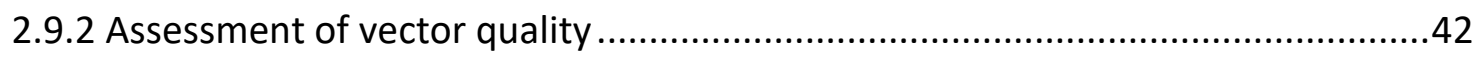

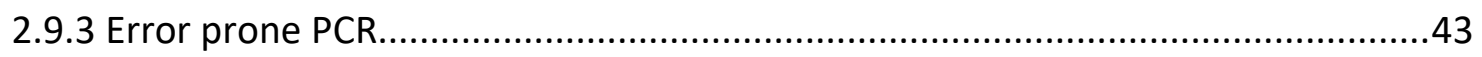

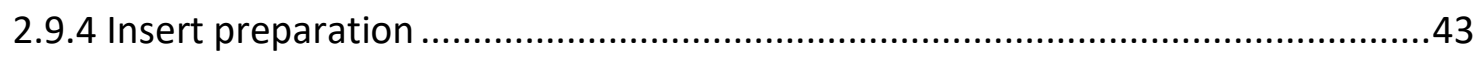

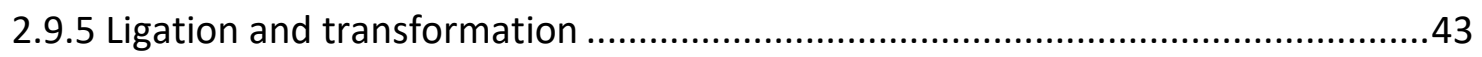

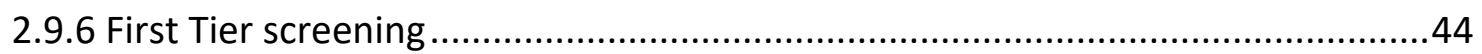

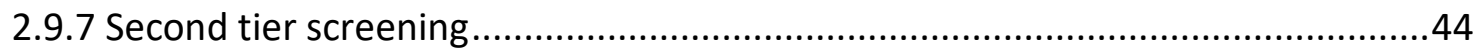

2.9.8 Quantification and normalisation of indigoidine levels..................................45

2.10 In vitro L-glutamine and ATP assays..............................................................45

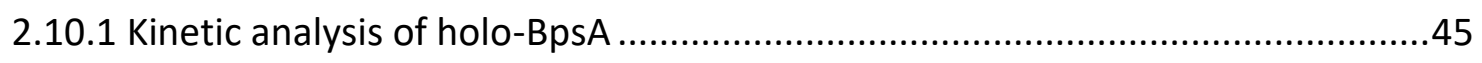

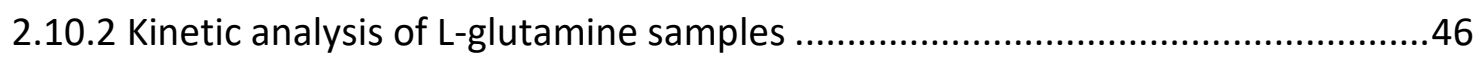

2.10.3 Optimisation of DMSO stop method .........................................................

2.10.4 Measurement of glutamine in a range of relevant conditions.........................46

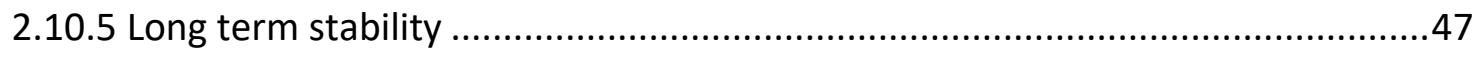

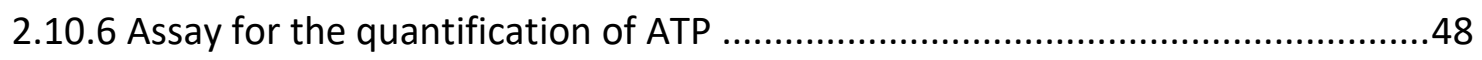

2.11 Assays for the detection of adenylation ........................................................ 48

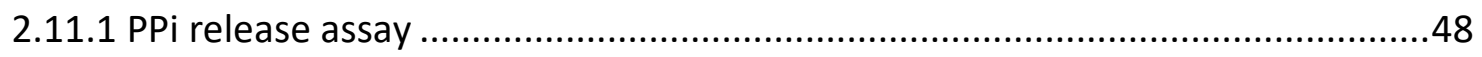

2.11.2 Quantification of adenylation by monitoring the consumption of ATP ............49

2.12 Synthesis and quantification of glutamine analogues .........................................49

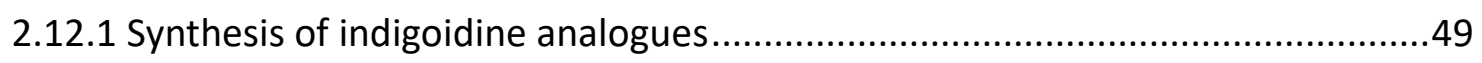

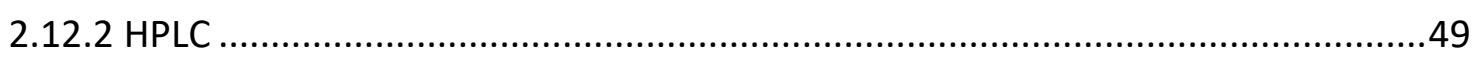

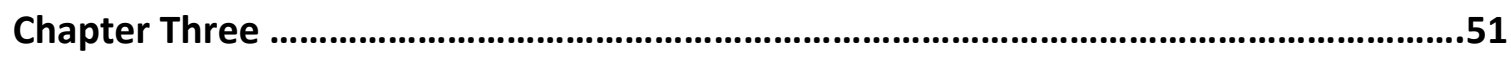

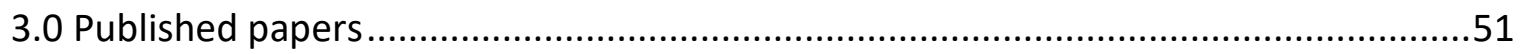




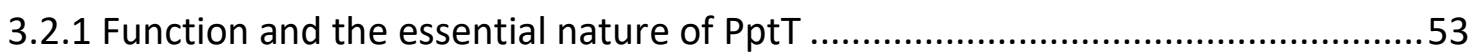

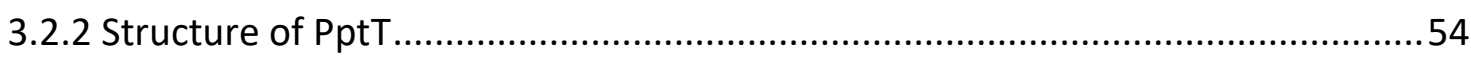

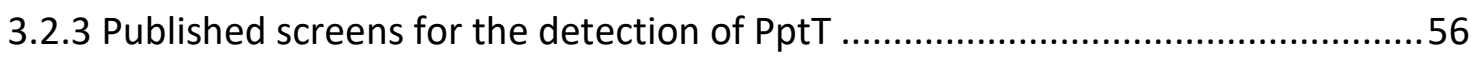

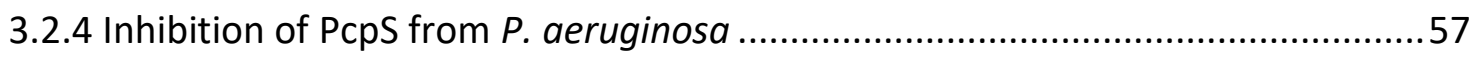

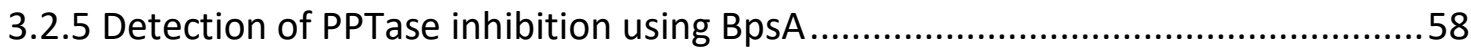

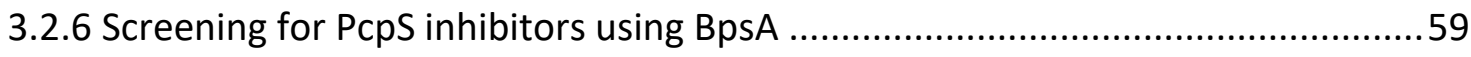

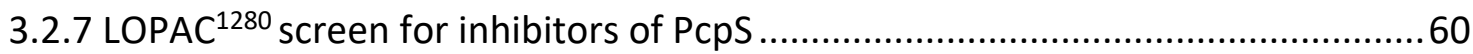

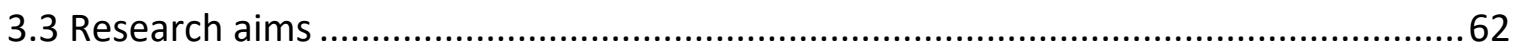

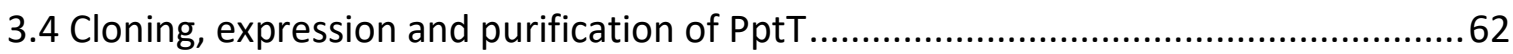

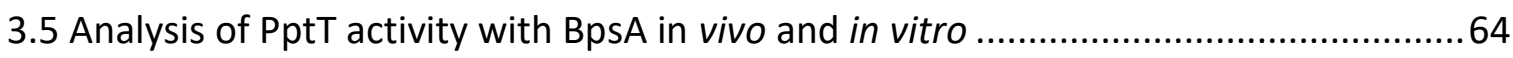

3.6 Development and optimisation of an assay to detect PptT inhibition ......................66

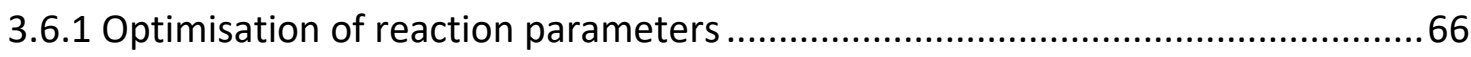

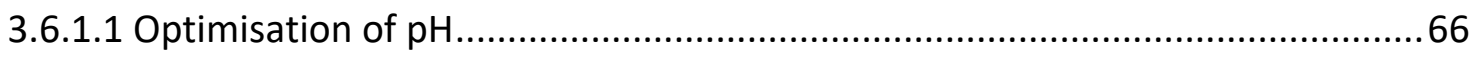

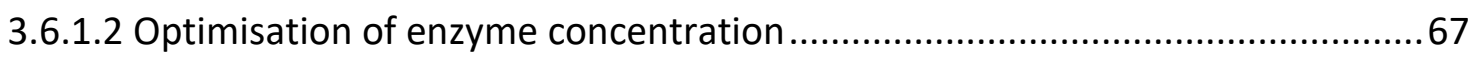

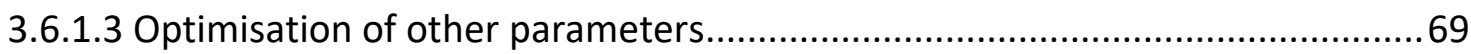

3.6.2 Quantification of PptT inhibition with 6-NOBP .............................................. 70

3.7 Development of a high throughput assay to detect the inhibition of PptT ................71

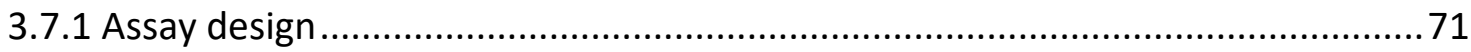

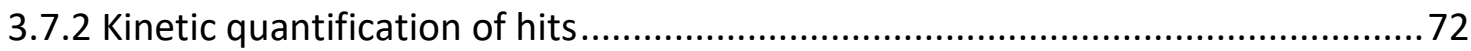

3.8 Identification of PptT inhibitors from the NIH Diversity Set II.................................74

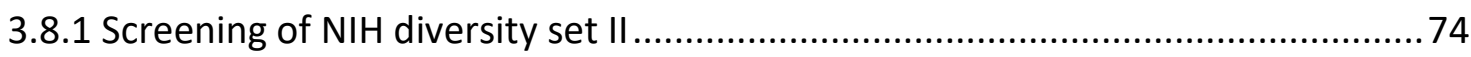

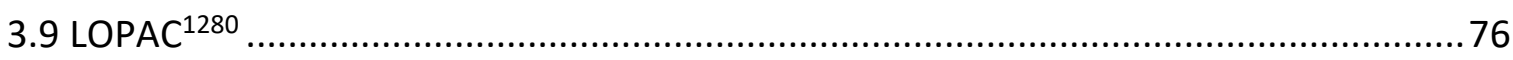

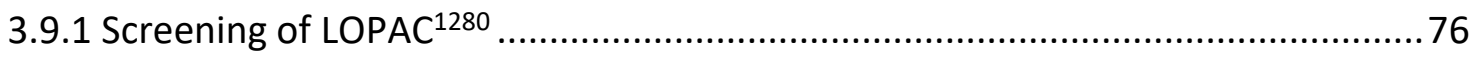

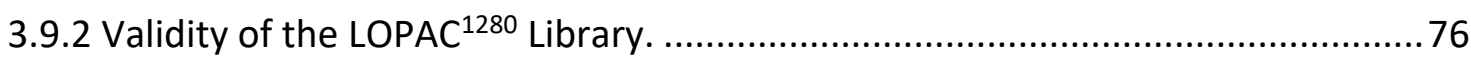




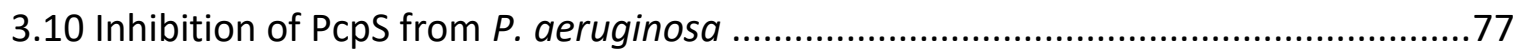

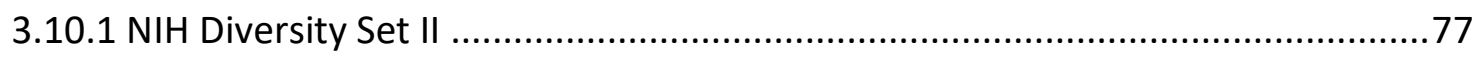

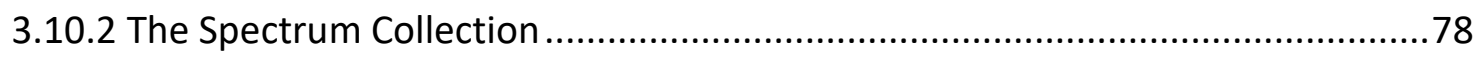

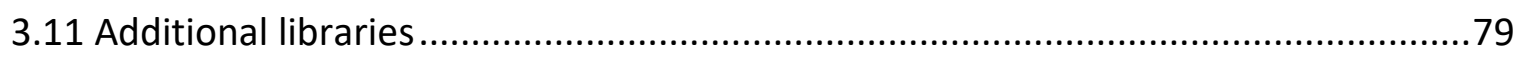

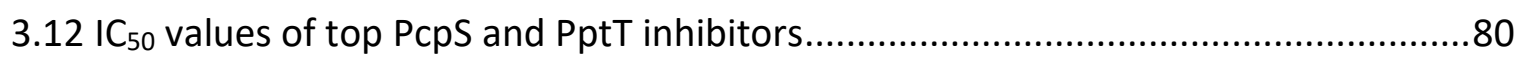

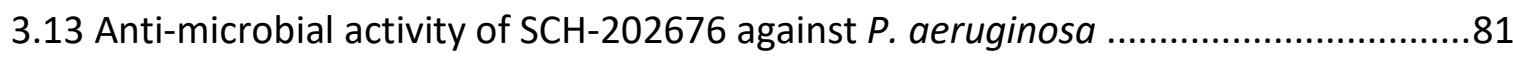

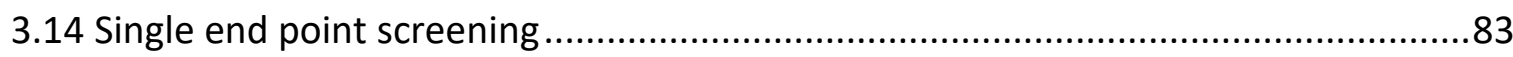

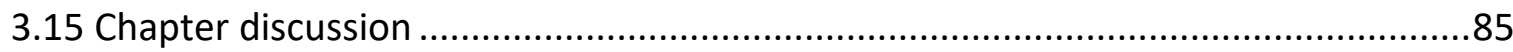

3.15.1 Development of a screen to detect inhibitors of PptT .....................................85

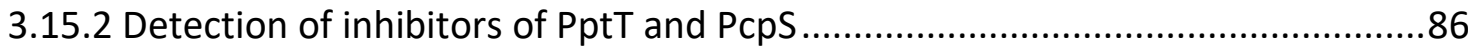

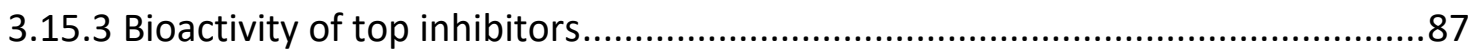

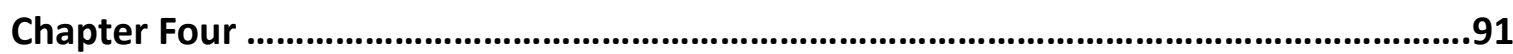

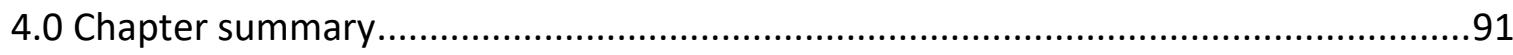

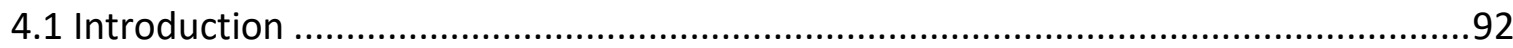

4.1.1 Structure and Function of AcpS in M. tuberculosis.........................................92

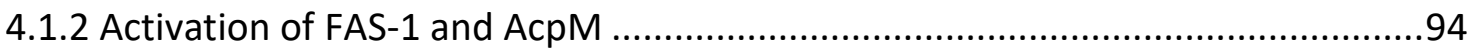

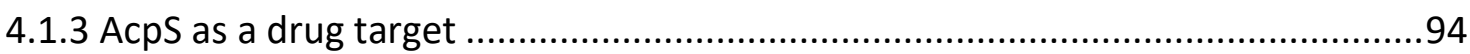

4.1.4 Engineering PCP domains for improved activity with type I PPTases .................96

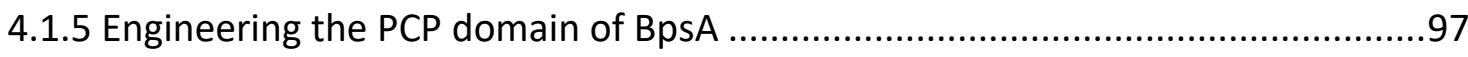

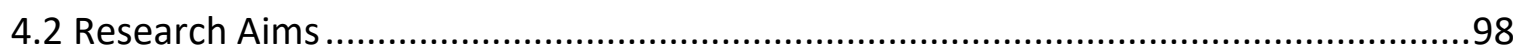

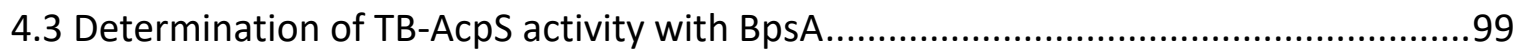

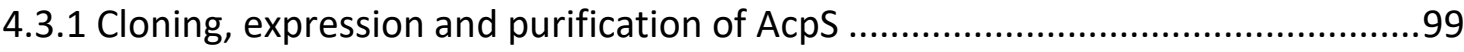

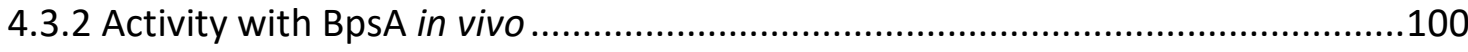

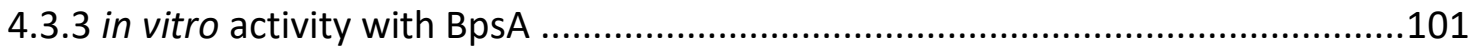

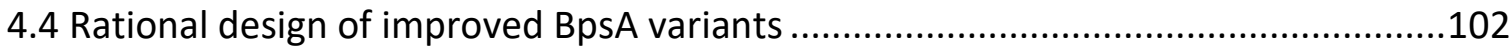

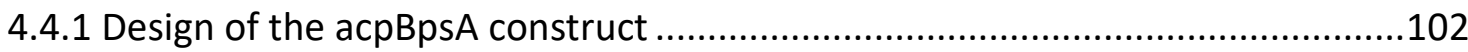


4.4.2 Construction of acpBpsA 103

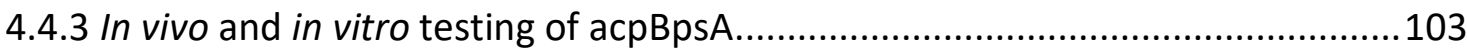

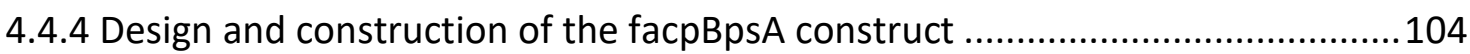

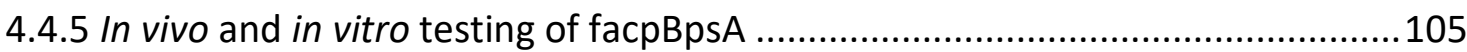

4.5 Development of pilot libraries for directed evolution...........................................106

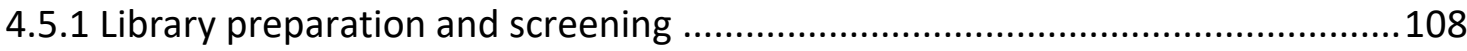

4.5.2 Sequence analysis of the selected first round variants ...................................109

4.6 Construction and screening of the second round library ......................................111

4.6.1 Second tier screening of second round library ...........................................111

4.6.2 Sequence analysis of the second round library .............................................112

4.7 Construction and screening of the third round library...........................................114

4.7.1 Second tier screening of third round library ...............................................114

4.7.2 Sequence analysis of the top variants from round three ...............................115

4.8 In vitro testing of top variants from each round of evolution..................................117

4.8.1 Expression and purification of top variants.................................................119

4.8.2 In vitro kinetics of variants in their purified form........................................120

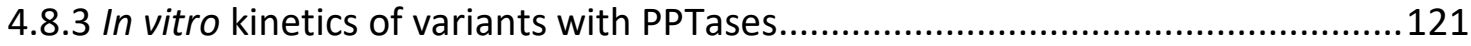

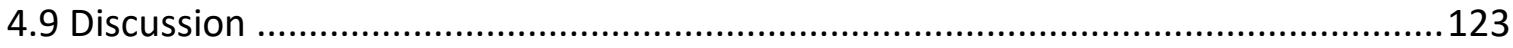

4.9.1 Selection and development of rational designed constructs ...........................123

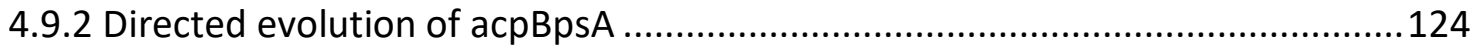

4.9.3 Critical evaluation of the directed evolution process......................................125

4.9.4 Additional improvements to the experimental procedures in the chapter .......127

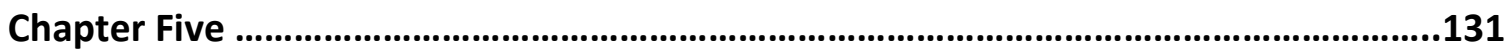

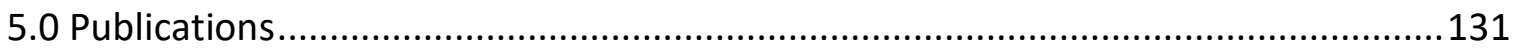

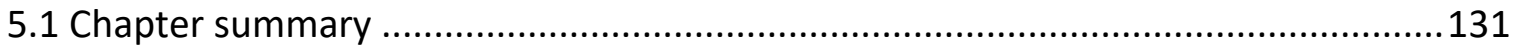

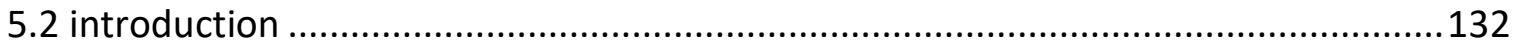




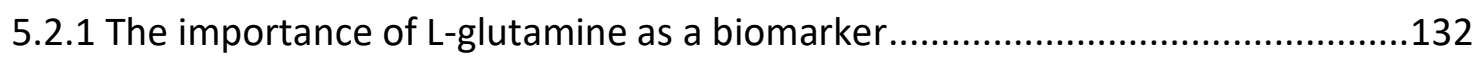

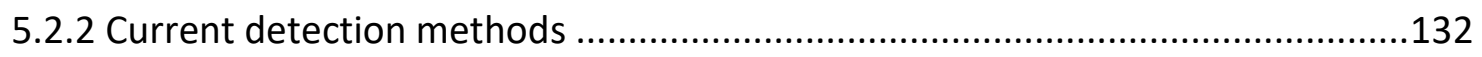

5.2.3 Using BpsA as a biosensor for the detection of L-glutamine..........................133

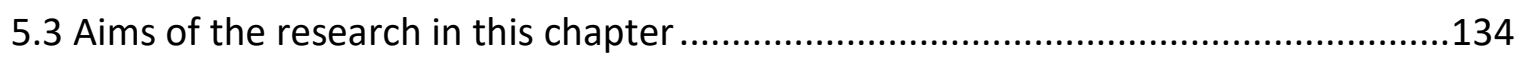

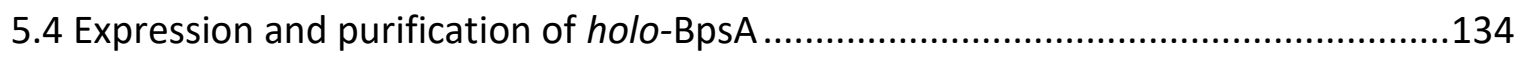

5.4.1 in vitro conversion of BpsA from the apo to the holo form .............................135

5.4.2 In-lysate conversion of BpsA from the apo to the holo form ...........................135

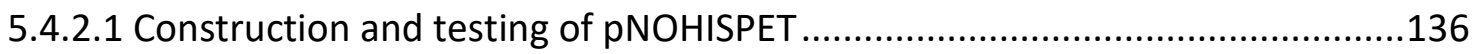

5.5. Development of an assay to quantify indigoidine production ..............................137

5.6 Development of a two-step resolubilisation protocol............................................138

5.6.1 Optimisation and miniaturisation of indigoidine solubilisation protocol...........140

5.7 Assay performance in common laboratory growth media....................................143

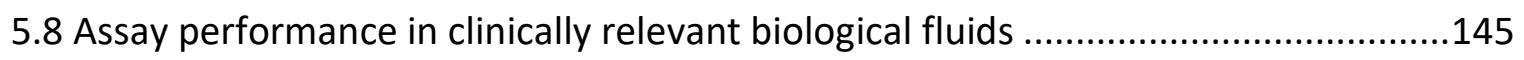

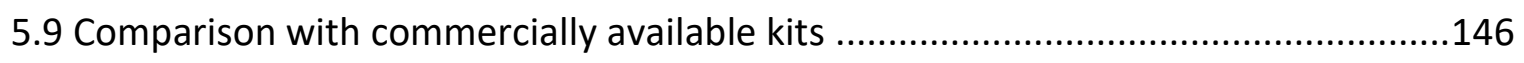

5.10 Alternative methods for the detection of L-glutamine .....................................147

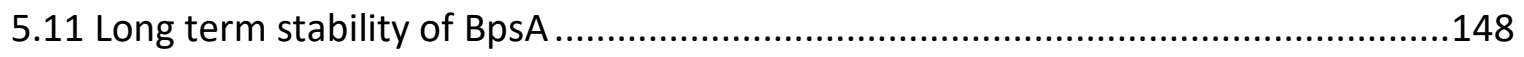

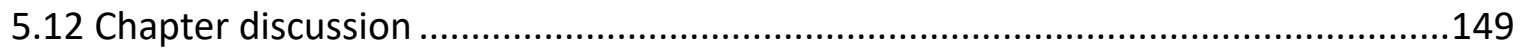

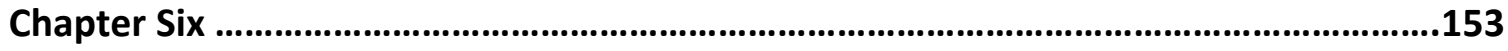

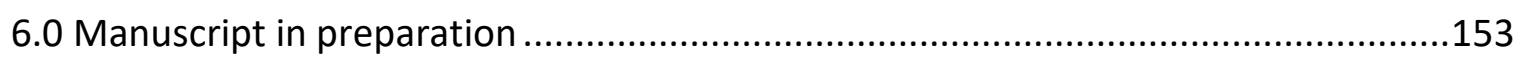

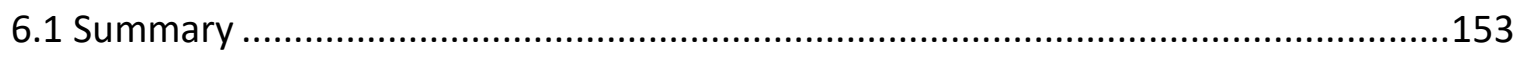

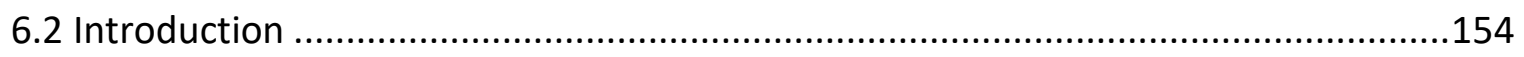

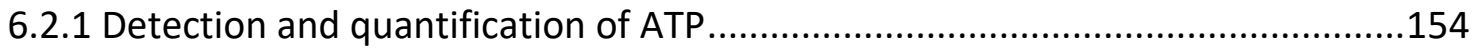

6.2.2 Detection of adenylation by A domains ..................................................... 156

6.3 Development of an assay to detect and quantify ATP .........................................157

6.4 Quantification of ATP levels in different cell culture media ....................................159

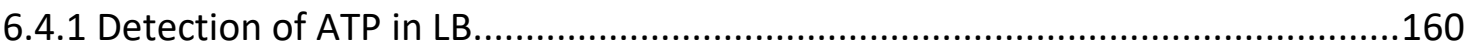


6.4.2 Establishment of standard curves using the media being tested as a vehicle ...160

6.5 Detection of adenylation by an NRPS A domain 165

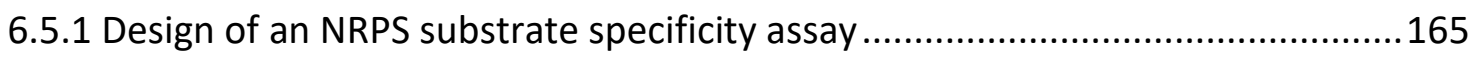

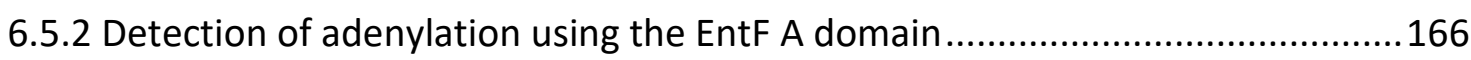

6.6 Synthesis and detection of a novel indigoidine analogue ..................................170

6.6.1 Synthesis of an indigoidine analogue from alanyl-glutamine ........................171

6.6.2 Detection of the indigoidine analogue using HPLC .....................................174

6.7 Investigation of 4' oxalocrotonate tautomerase mediated conversion of indigoidine

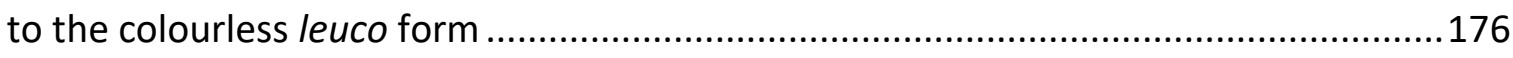

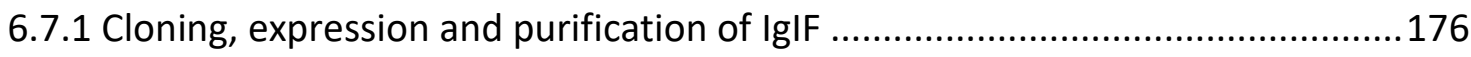

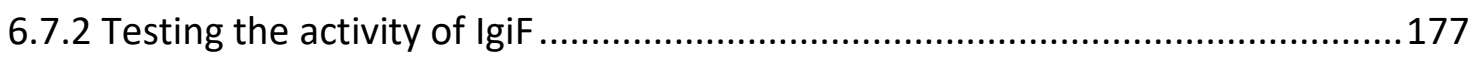

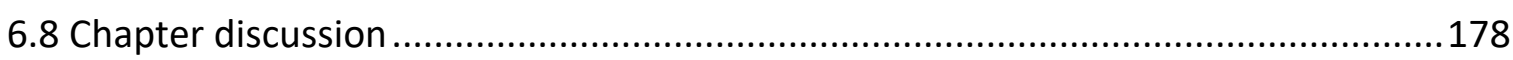

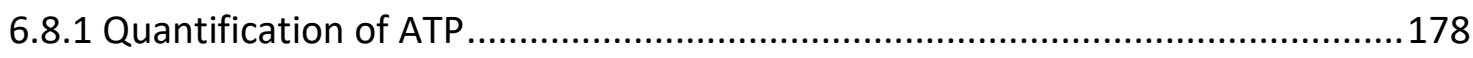

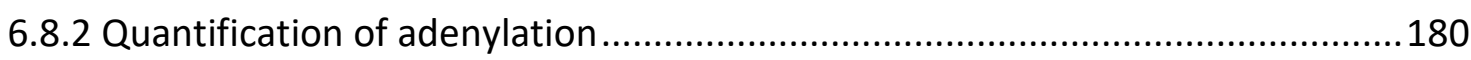

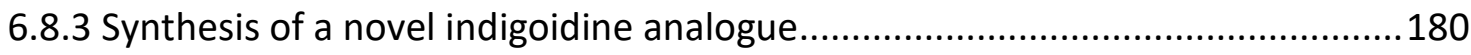

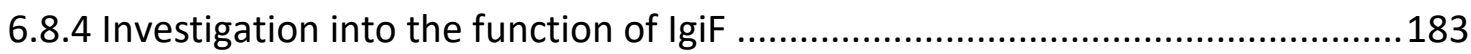

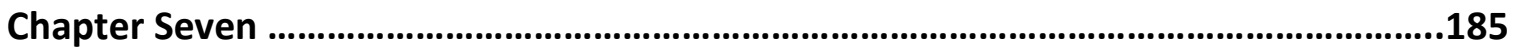

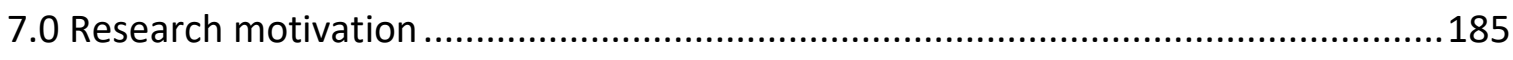

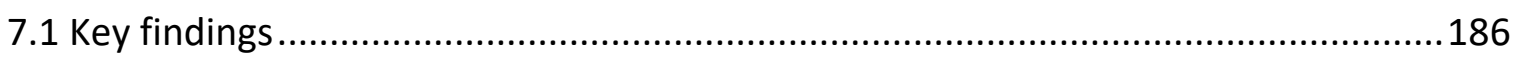

7.1.1 Development of an assay to detect PPTase inhibition ..................................186

7.1.2 Evolution of the PCP domain of BpsA to recognise type I PPTases ...................187

7.1.3 Development of a biosensor to quantify L-glutamine....................................188

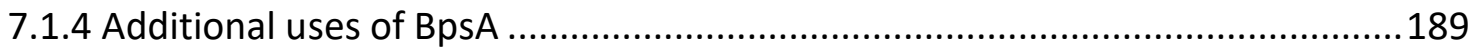

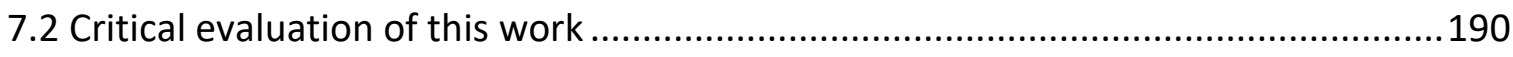

7.2.1 Development of HTS to assay to detect PPTase inhibition ..............................190

7.2.2 Evolution of the PCP domain of BpsA to accept type I PPTases.......................191 
7.2.3 Development of a biosensor to quantify L-glutamine .191

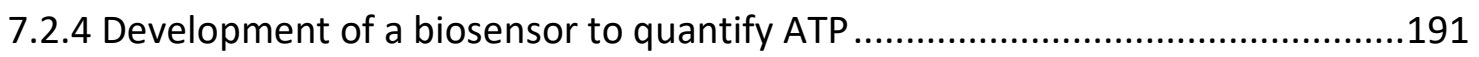

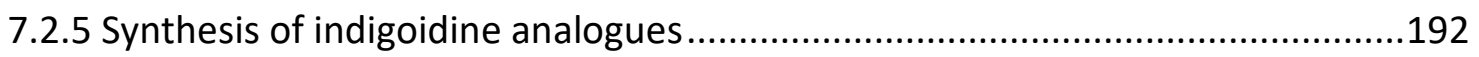

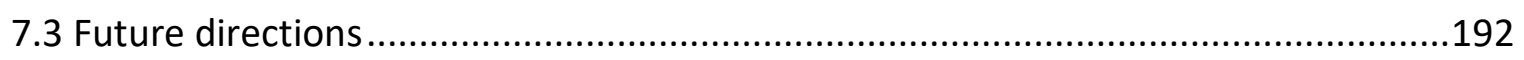

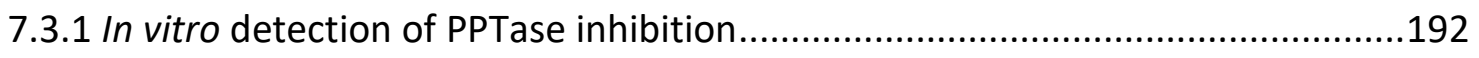

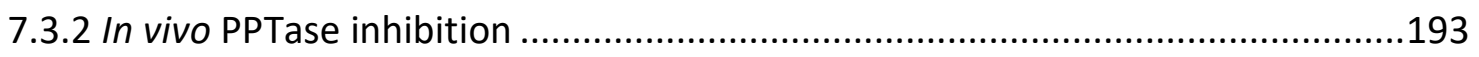

7.3.3 Evolution of the BpsA PCP domain for activity with type I PPTases ...................194

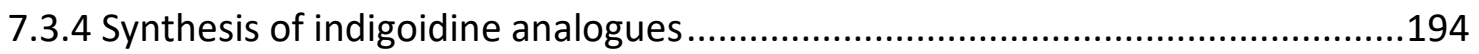

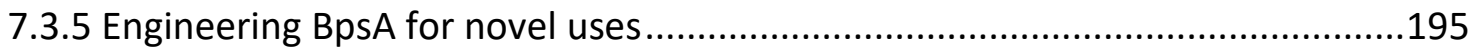

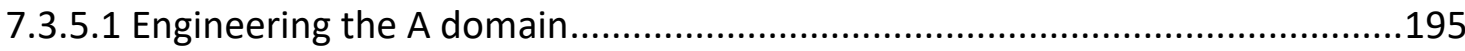

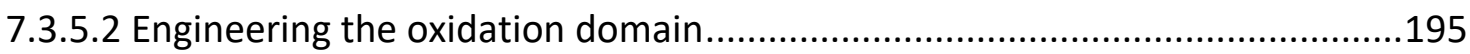

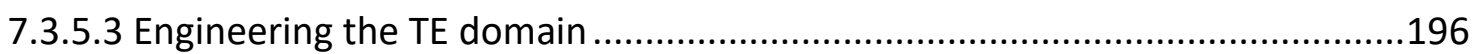

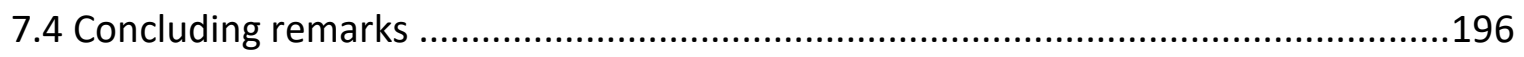

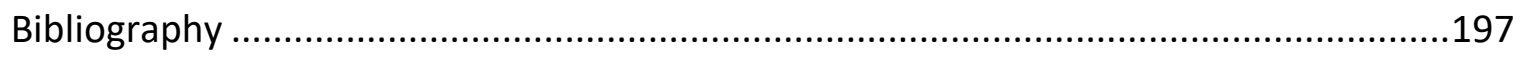




\section{List of Figures}

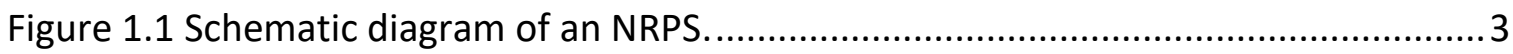

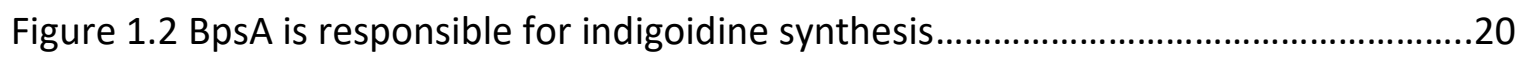

Figure 1.3 Deriving PPTase kinetic parameters using BpsA as a reporter...........................24

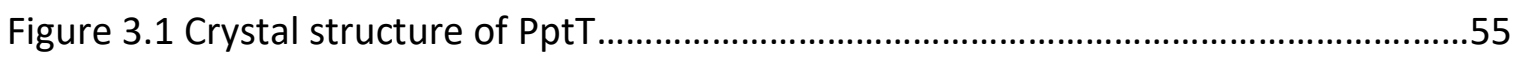

Figure 3.2 Schematic diagram illustrating BpsA being used to detect inhibition ................59

Figure 3.3 A 12\% SDS-PAGE gel illustrating the optimised purification protocol...............64

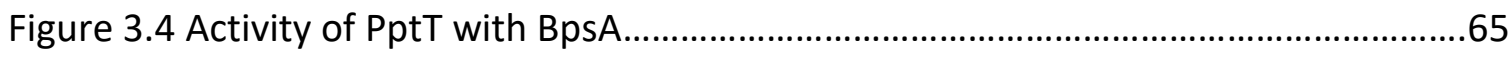

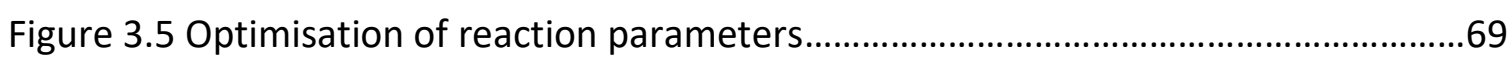

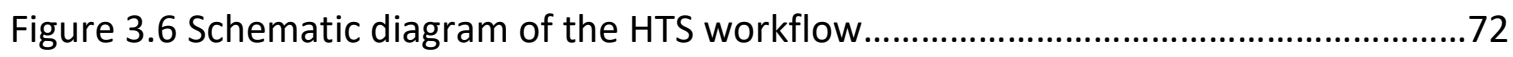

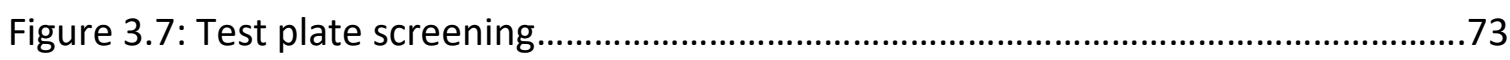

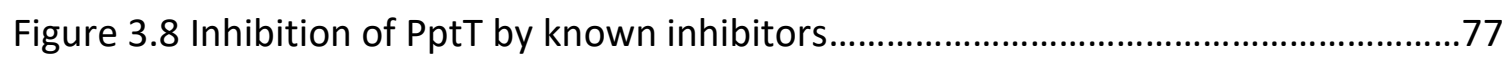

Figure 3.9 Anti-microbial activity of SCH-202676 against P. aeruginosa............................83

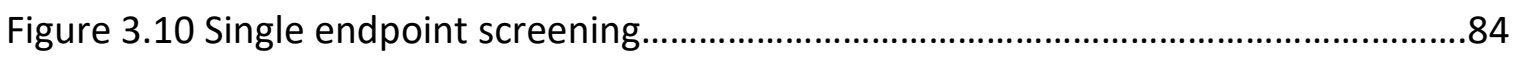

Figure 4.1 Structure of TB-AcpS compared to AcpS from B. subtilis..................................93

Figure 4.2 SDS-Page gel showing the TB-AcpS purification protocol ..............................100

Figure 4.3: Activation of BpsA by TB-AcpS both in vivo and in vitro..................................102

Figure 4.4 Alignment of the second alpha helix of AcpM with the second alpha helix of

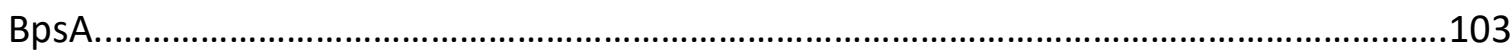

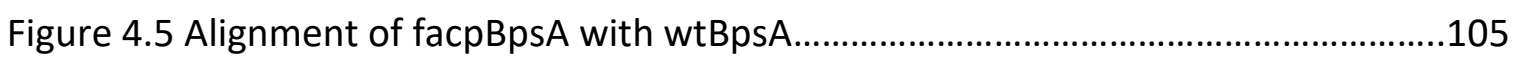

Figure 4.6 In vitro activity of acpBpsA and facpBpsA with TB-AcpS and PptT..................106

Figure 4.7 Schematic diagram of the directed evolution campaign ...................................107

Figure 4.8 Sequence alignment of top-selected variants from the first round of directed

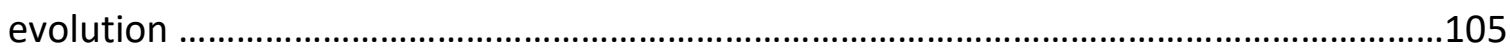


Figure 4.10 Sequence alignment of top-selected variants from the second round of directed evolution. .107

Figure 4.11 Results from third round evolution. .108

Figure 4.12 Sequence alignment of the top selected variants from the third round of directed evolution

Figure 4.13 Variants selected for in vitro kinetics

Figure 4.14. Qualitative assessment of indigoidine production following 16 hours by $E$. coli $\Delta$ entD BL21 cells expressing the first and second round variants.

Figure 4.15. Synthesis of indigoidine by acpBpsA variants purified in vitro...

Figure 4.16 Synthesis of indigoidine by RD3_Mut_11 activated by different PPTases...113

Figure 5.1 Schematic diagram showing two molecules of L-glutamine being converted into one molecule of the blue pigment indigoidine by holo BpsA.

Figure 5.2 Conversion of BpsA to the holo form using PcpS.

Figure 5.3 Direct monitoring of indigoidine synthesis does not yield a standard curve. 128

Figure 5.4: Resolubilisation of indigoidine in DMSO 130

Figure 5.5. Optimisation of assay incubation steps. .131

Figure 5.6 Generation of a standard curve using the optimised protocol 132

Figure 5.7 Measurement of L-glutamine concentrations in laboratory media and clinical relevant samples .134

Figure 5.8 Converting indigoidine to its colourless leuco form does not provide a more accurate means of measuring L-glutamine. .138

Figure 5.9 Effect of long-term storage on BpsA activity. 139

Figure 6.1 Schematic diagram illustrating the synthesis of indigoidine by BpsA 145 
Figure 6.2 Optimisation and determination of reaction parameters for the ATP assay

Figure 6.3 Comparison of standard curves between ddH2O, RPMI and LB

Figure 6.4 Comparison of reaction velocity of a sample supplemented with $25 \%$ LB vs no supplementation

Figure 6.5 Maximum reaction velocity for LB components and the effect of autoclaved vs non-autoclaved $L B$ on reaction velocity. 154

Figure 6.6 The effect of the addition of $5 \mathrm{mM}$ ascorbic acid on total indigoidine production. .155

Figure 6.7 Determination of the EntF A domain construct solubility and activity .157

Figure 6.8 First attempt to measure adenylation by monitoring the consumption of ATP. .158

Figure 6.9 The addition of pyrophosphatase results in detectable adenylation 159

Figure 6.10: Structures of glutamine, alanyl-glutamine, Indigoidine and proposed indigoidine analogue.

Figure 6.11 different in the synthesis patterns of indigoidine synthesised from glutamine and alanyl-glutamine

Figure 6.12 HPLC trace of indigoidine and ala-indigoidine

Figure 6.13 Conversion of indigoidine to leuco-indigoidine is potentially mediated by IgiF.....

Figure 6.14: Analogues of indigoidine identified in different bacterial strains. .171 


\section{List of tables}

Table 2.1 Bacterial strains used in study

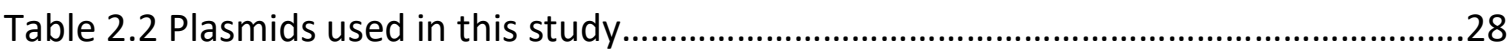

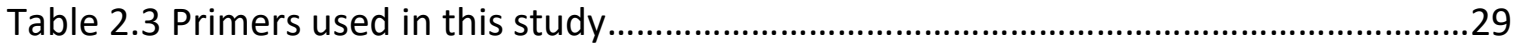

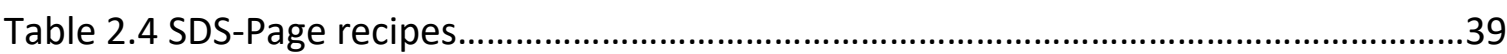

Table 3.1 Top inhibitors identified from the LOPAC ${ }^{1280}$ screen...........................................60

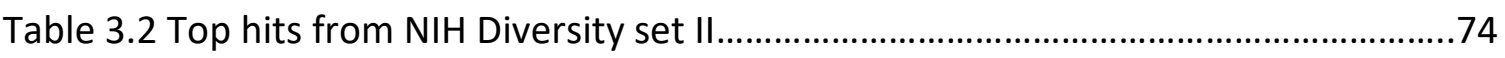

Table 3.3 Top inhibitors identified from NIH Diversity set II............................................77

Table 3.4 Top inhibitors identified from the Spectrum Collection.......................................78

Table $3.5 \mathrm{IC}_{50}$ values of top inhibitors identified across the three libraries.........................79

Table 5.1. Measurement of L-glutamine in spiked laboratory media and biological samples 


\section{List of Abbreviations}

$6 \mathrm{His}$

$A_{590}$

A domain

ACP

ACPS

APS

ATP

BLAST

BSA

bp

BpsA

BSA

C domain

CoA

$\mathrm{CP}$

$\mathrm{ddH}_{2} \mathrm{O}$

DMEN

DMSO

DTP

E domain

EM

eDNA

EDTA

EX

FAS

FP

HTS

FMN six histidine expression tag

absorbance at $590 \mathrm{~nm}$

adenylation domain

acyl carrier protein

acyl carrier protein synthase

ammonium persulfate

adenosine triphosphate

basic Local alignment search tool

bovine serum albumin

basepair

blue pigment synthetase $A$

bovine serum albumin

condensation domain

coenzyme A

carrier protein

double distilled water

dulbecco's modified eagle's medium

dimethylsulfoxide

development therapeutics programme

epimerisation domain

emission

environmental DNA

ethylenediaminetetraacetic acid

excitation

fatty acid synthase

fluorescent polarisation

high throughput screening

flavin mononucleotide 
h

HS-Ppt

${ }^{I} C_{50}$

IP

IPTG

$\mathrm{kb}$

$k_{\text {cat }}$

$K_{m}$

KO

LB

$\min$

MIC

MCDB

MCS

MDR

NIH

NRPS

$\mathrm{OD}_{590}$

$\mathrm{OD}_{600}$ hour

human PPTase

concentration when $50 \%$ inhibition occurs

inorganic pyrophosphatase

isopropyl $\beta$-D-1-thiogalactoside

kilobase

kinetic constant describing theoretical maximum rate of catalysis for a specific enzyme with a specific substrate, under the conditions tested.

kinetic constant describing the concentration of substrate at which rate of catalysis of a specific enzyme is exactly half of the $V_{\max }$, under the conditions tested.

knockout

luria broth

minute

minimum inhibitory dose

molecular, cellular, and developmental

biology

multiple cloning site

multidrug resistant

national institute of health

non-ribosomal peptide synthetase

optical density at $590 \mathrm{~nm}$

optical density at $600 \mathrm{~nm}$ 


\begin{tabular}{|c|c|}
\hline $\mathrm{OD}_{800}$ & optical density at $800 \mathrm{~nm}$ \\
\hline ORF & open reading frame \\
\hline PCP & peptidyl carrier protein \\
\hline $\mathrm{Pi}_{\mathrm{i}}$ & $\mathrm{PO}_{42}$ - (inorganic phosphate) \\
\hline $\mathrm{PP}_{\mathrm{i}}$ & $\mathrm{P}_{2} \mathrm{O}_{7}^{4-}$ (pyrophosphate) \\
\hline Ppant & 4'-phosphopantetheine \\
\hline PPTase & 4'- phosphopantetheinyl transferase \\
\hline PKS & polykeytide synthetase \\
\hline PSI & pounds per square inch \\
\hline $\mathrm{Rev} / \mathrm{min}$ & revolutions per minute \\
\hline RPMI & roswell park memorial institute \\
\hline$S$ & seconds \\
\hline SD & standard deviation \\
\hline SEM & standard error of the mean \\
\hline SPA & scintillation proximity assay \\
\hline TB & tuberculosis \\
\hline TMED & tetramethylethylenediamine \\
\hline$u$ & arbitrary units \\
\hline UV & ultraviolet \\
\hline v & volts \\
\hline$v / v$ & volume/volume \\
\hline WHO & world health organisation \\
\hline wtBpsA & wild-type BpsA \\
\hline$w / v$ & weight/volume \\
\hline XDR & extremely drug resistant \\
\hline
\end{tabular}




\section{Chapter 1 - Introduction}

\subsection{Non-ribosomal peptide synthetases}

Non-ribosomal peptide synthetases (NRPSs) are large, often multi-modular enzymes that are present in many bacteria and fungi (Wang et al., 2015). NRPSs are responsible for the production of a range of peptide secondary metabolites that confer a growth advantage under certain conditions (Finking and Marahiel, 2004). Peptides produced by NRPSs are structurally diverse and have range of pharmacologically relevant bioactivities including antibiotics (e.g., penicillin and vancomycin), anti-cancer agents (e.g., bleomycin A2) and immunosuppressants (e.g., cyclosporine A) (Schwarzer et al., 2003).

NRPSs are structurally and evolutionarily distinct to the ribosomal mechanism for peptide synthesis. The ribosomal system is ubiquitous across all kingdoms of life and is able to rapidly synthesise proteins from the instructions present in mRNA. Due to the essential role it plays in protein synthesis, ribosomes contain several different proofreading mechanisms. Despite their accuracy and speed, they have some limitations, for example they are only able to recognise and process up to 22 proteogenic amino acids and they are unable to post-translationally modify their products, e.g. by methylation or cyclisation (Finking and Marahiel, 2004; Gewolb, 2002). In contrast, NRPSs typically lack the level of proofreading required for primary synthesis. They do however have several advantages over the ribosome, the main one being that they are not limited to the proteogenic amino acids. NRPSs can incorporate a range of monomers including both $D$ and $\beta$ amino acids, $\alpha$-keto and $\alpha$-hydroxy acids and fatty acids (Caboche et al., 2010; Marahiel et al., 1997) which greatly increases the diversity of products synthesised Over 500 different monomers have been identified (Walsh et al., 2015). In NRPSs the proofreading capabilities are handled by the different domains which, means that while NRPSs are not as accurate as ribosomes they are not limited to the small pool of proteogenic amino acids. 


\subsection{NRPS function}

NRPSs are structurally organised in a manner similar to an assembly line. Typically, several modules are connected together, with each module being made up of multiple domains that have different functions. In the most common (non-iterative) NRPS systems, each module is responsible for the addition of a single monomeric substrate into a growing peptide chain. Thus, in most cases the number of modules in an NRPS corresponds to the number of monomers in the product. With the exception of the first (initiation) module in an NRPS complex, which usually lacks a condensation domain (C domain), the core domains in each module usually consist of a $\mathrm{C}$ domain, an adenylation domain (A domain) and a peptidyl carrier protein domain (PCP domain), also known as a thiolation domain (T domain). The final (termination) module in an NRPS also contains a thioesterase domain (TE domain) that catalyses the removal of the mature peptide from the NRPS. (Konz and Marahiel, 1999; Stachelhaus et al., 1998). NRPSs are posttranslationally activated by a 4'phosphopantetheinyl transferase (PPTase) enzyme partner, which attaches a 4'-phosphopantetheine (Ppant) arm to the PCP domain. This converts the NRPS from the in-active apo state to the active holo state (Lambalot et al., 1996). The movement of the peptide chain and interaction of the NRPS domains are illustrated in Fig. 1.1. In addition to the basic C, A and PCP domains modules can also include a variety of tailoring domains. These modules are discussed in more detail in the following sections. 


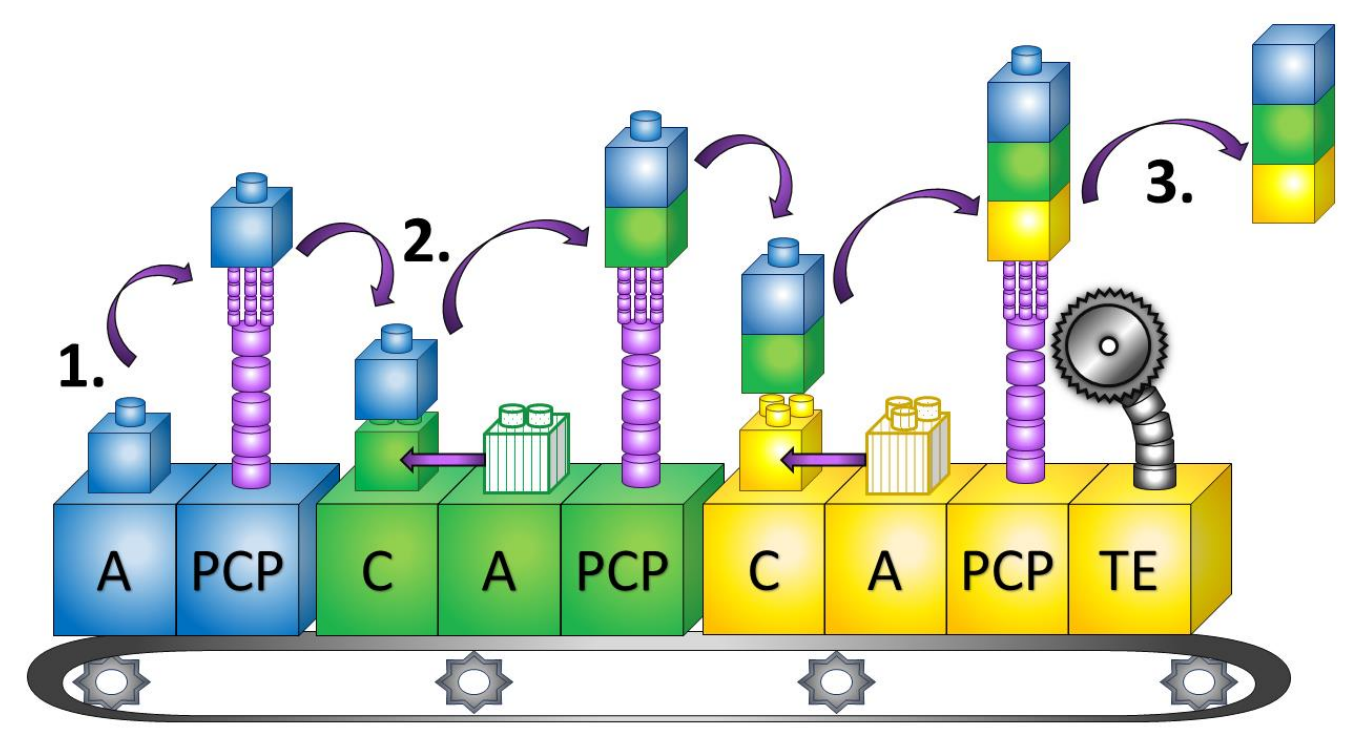

Figure 1.1 Schematic diagram of an NRPS: Three modules are illustrated, an initiation module consisting of an $A$ and PCP domain, an elongation domain consisting of a C, A and PCP domain and a termination domain consisting of a C, A, PCP and TE domain. (1) A monomer is adenylated by the A domain which is tethered to the free sulfhydryl of the Ppant arm (represented in purple) attached to the downstream PCP domain. (2) The C domain condenses the upstream monomer with the downstream substrate. This reaction breaks the upstream thioester bond which yields a peptidyl intermediate attached to the Ppant arm of the downstream PCP domain. The peptidyl intermediate then acts as a donor substrate for the downstream PCP domain and the elongating peptide chain is passed down the modules of the NRPS until it reaches the termination module. (3) After the termination module has added the final peptide to the chain the TE domain then catalyses the release of the mature peptide. This image is adapted from (Ackerley, 2016) and was originally drawn by Jasmine Chan-Hyams.

\subsection{Core NRPS domains}

\subsubsection{The adenylation domain}

The adenylation domain (A domain) is approximately $60 \mathrm{kDa}$ in size and is responsible for the first step in NRPS synthesis (Gulick, 2009). A domains catalyse two reactions, the adenylation of a specific monomer substrate in an ATP powered reaction, and then the loading of this activated monomer via a thioester bond with the Ppant arm covalently bonded to the PCP domain (Gulick, 2009).

Several different A domain structures have been elucidated. The first structure to be solved was the A domain from the gramicidin synthetase module PheA, which activates L-phenylalanine (Conti et al., 1997). A domains are structurally organised into two subdomains, a core subdomain at the $\mathrm{N}$-terminus and a smaller subdomain at the $\mathrm{C}$ terminus. These are linked by a flexible hinge region, with the active site located at the interface. Despite only sharing $16 \%$ amino acid identity, each of the sub-domains have 
similar structure to unbound firefly luciferase, another adenylate forming enzyme (Conti et al., 1997; Süssmuth and Mainz, 2017).

Landmark research by Stachelhaus et al (1999) identified 8 important residues in the active site of $A$ domains that collectively confer specificity for a monomer. By comparing the residues of the binding pocket of the PheA with the corresponding moieties identified in other A domains they were able to develop general rules, now usually referred to as the Stachelhaus code, that allow the in-silico prediction of substrate specificity (Stachelhaus et al., 1999). Further refinement and improvements in algorithms have given rise to a range of software packages able to predict $A$ domain specificity with a moderate degree of accuracy (Ansari et al., 2004; Röttig et al., 2011).

\subsubsection{The peptidyl carrier protein domain}

The PCP domain (or T domain) is a dynamic domain that is responsible for shuttling the intermediates between the NRPS domains and modules. At approximately $10 \mathrm{kDa}$ it is the smallest domain. PCP domains have two states, an inactive apo form and an active holo state where the Ppant arm is bound to a conserved serine located at the $\mathrm{N}$ terminus of the second alpha helix. Following attachment of the Ppant arm by a PPTase, the Ppant moiety acts as a swinging arm (Fig. 1.1) that covalently binds and transfers the amino acid and growing peptide intermediates and moves them between the different catalytic domains (Conti et al., 1997; Kittilä et al., 2016).

The first PCP domain structure to be elucidated was the third PCP domain from $B$. brevis tyrocidine synthetase 3 . Since then the structures of several other PCP domains have been solved, with only minor structural differences being identified (Kittilä et al., 2016). PCP domains adopt a four-helix bundle form and exhibit a similar structure to acyl carrier proteins from fatty acid synthetases and ACPS from polyketide synthetases (Haslinger et al., 2015b; Weber et al., 2000). The second alpha helix of the PCP domain contains the invariant serine and plays a role in recognition by the PPTase (Mercer and Burkart, 2007). PCP domains and the essential nature of PPTases for cell viability are discussed in more detail in Chapter Four. 


\subsubsection{The condensation domain}

Condensation domains ( $\mathrm{C}$ domains) are approximately $50 \mathrm{kDa}$ in size and catalyse the coupling reaction of an upstream monomer or peptide with a downstream monomer. In addition, $\mathrm{C}$ domains have a key role in translocating the growing peptide to the downstream PCP domain, and as functioning as a "gatekeeper" that contributes to the specificity of a module for the downstream monomer. The condensation domain was identified in 1995 by De Crecy-Lagard et al (De Crecy-Lagard et al., 1995). Biochemical evidence of the condensation domain function was provided by Stachelhaus et al in 1998 who showed that the $C$ domain was responsible for the condensation reaction in NRPSs (Stachelhaus et al., 1998).

The first crystal structure of the $C$ domain was published in 2002 by Keating et al. They crystallised a standalone $\mathrm{C}$ domain named VibH from Vibrio cholerae, which has a role in the synthesis of a siderophore, vibriobactin. The $\mathrm{C}$ domain consists of two lobes which form a head-to-tail pseudo dimer (Keating et al., 2002). The two lobes form a central cleft at the interface, where the donor and acceptor Ppant arms enter and interact from opposite sides to reach the active site at the centre (Bloudoff and Schmeing, 2017; Süssmuth and Mainz, 2017). Like A domains, C domains also exhibit a specificity for substrates. It appears that $\mathrm{C}$ domains have higher levels of specificity at the downstream acceptor site than the upstream donor site, with selectivity for both amino acid side chain and chirality at the acceptor site and selectivity only for chirality at the donor site (Belshaw et al., 1999; Luo et al., 2002).

\subsubsection{The thioesterase domain}

The thioesterase domain (TE domain) is approximately $30 \mathrm{kDa}$ in size and is responsible for releasing the mature peptide product from an NRPS by cleaving the thioester bond connecting it to the terminal PCP domain (Bruner et al., 2002). TE domains are only found in the final NRPS termination module (containing at minimum a $C, A, P C P$ and TE domain). The structure of the TE domain from SrfA-C of Bacillus subtilis is globular in structure and has a distinct bowl shaped hydrophobic cavity that holds the acyl-peptide substrate and permits its cyclisation (Bruner et al., 2002). 
Most TE domains exclude water from the active site and catalyse an internal cyclisation of the peptide product rather than hydrolytic cleavage of the mature peptide (Samel et al., 2006). However, some TE domains can release a linear product, moreover some TE domains such as the gramicidin S synthetase TE domain can also act as a waystation for the accumulation of identical peptide units before these are condensed and cyclised and ultimately released by the TE domain (Keating et al., 2001).

\subsection{Additional tailoring domains}

In addition to the core $\mathrm{C}, \mathrm{A}, \mathrm{T}$ and TE domains, NRPSs can contain additional tailoring domains that modify the substrate they are incorporating, which helps to contribute to the rich diversity of products produced. Some of the most common domains are discussed here.

A variety of NRPS products contain $N$-methyl amino acids. The domain responsible for this modification is called a methyltransferase domain (MT domain) (Walsh et al., 2001). $N$-methylation can have an effect on polarity and hydrogen-bonding ability (Chatterjee et al., 2013). One extreme example of $\mathrm{N}$-methylation is the non-ribosomal peptide cyclosporin, in which 7 of the 11 amino acids are $N$-methylated (Weber et al., 1994). The MT domain is usually located downstream of the A domain (A-MT-PCP) (Schauwecker et al., 2000), however it can also lie embedded within the A domain as is the case of the PchF subunit, which is responsible for the synthesis of the siderophore pyochelin in Pseudomonas aeruginosa (Patel and Walsh, 2001).

Many non-ribosomal peptides contain D-amino acids. In these cases, A domains generally select the $L$ enantiomers of the monomer substrates and epimerisation ( $E$ domain) domains subsequently epimerise them once they have been bound to the Ppant arm (Walsh et al., 2001). However, some A domains are capable of recognising the D enantiomer of amino acids, for example the first monomer in the synthesis of cylcosporin is D-alanine, which is produced by a dedicated alanine racemase (Hoffmann et al., 1994). The E domain is always embedded within a module governing incorporation of a D-monomer into the peptide chain (Walsh et al., 2001). E domains are 
evolutionarily descended from $\mathrm{C}$ domains and have the same overall protein fold (Samel et al., 2014).

Another tailoring domain present in some NRPSs is the oxidation (OX) domain. This domain oxidises the monomer substrate and is found downstream of PCP domain or inserted into the $C$ terminus of the $A$ domain (Hur et al., 2012). Oxidation domains contain non-covalently bound Flavin mononucleotide (FMN) as a co-factor (Du et al., 2000). In addition to oxidation domains, cytochrome P450 enzymes that are present in bioactive clusters can also oxidise amino acids bound to PCP domains (Payne et al., 2017). Cytochrome P450 enzymes can be recruited to the NRPS by X domains. These domains are structurally related to the $C$ domain but have no catalytic activity. The NRPS machinery that synthesises well known glycopeptide antibiotics such as vancomycin uses $X$ domains to recruit cytochrome P450 enzymes which catalyse the cross-linking of the aromatic side chains giving the structure rigidity (Haslinger et al., 2015).

\subsection{NRPS higher order structure}

Recent structural NRPS structural studies have provided a wealth of knowledge about the dynamics of module and domain interactions and allowed us to shine a light on an otherwise poorly understood area of NRPS function. The first published image of multiple NRPS modules was a negative stain electron micrograph of the 11-module cyclosporin synthetase, which was published by Hoppert et al (2001). Cyclosporin synthetase appeared as a collection of elongated and globular particles, which gave rise to the idea that NRPS modules may function like 'beads on a string' without a higher order structure (Hoppert et al., 2001).

In contrast, several recent papers have started to paint a different picture. Marahiel proposed a more ordered helical structure in 2016. By integrating structural details of the different domains along with a PCP-C di-domain and an elongation module, he suggested that NRPS complexes might adopt a helical structure with a much more structured higher modular organisation (Marahiel, 2016).

Tarry et al (2017) published the first high resolution structure for a multi-modular NRPS using both X-ray crystallography and electron microscopy to analyse DhbF, a NRPS 
present in B. Subtilis involved in the synthesis of the siderophore bacillibactin Although their electron microscopy analysis of DhbF (with the TE domain removed) indicated a high level of conformational variability, and also a lack of consistent interfaces, between the modules, their crystallisation analysis of the A and PCP domains from module 1 and the $\mathrm{C}$ domain from module 2 nevertheless indicated that the relative orientation between domains within a module was consistent (Tarry et al., 2017). Other recent structural studies (Drake et al., 2016; Reimer et al., 2016) have also pointed towards the orientation between domains within a module being relatively consistent. Thus, although there doesn't appear to be a defined structure that exists between modules as proposed by Marahiel, there instead appears to be a high degree of flexibility between modules. Nonetheless, since the modules appear to remain in close proximity, there may be transient interactions that exist between them (Lott and Lee, 2017).

\subsection{The 4'- phosphopantetheinyl transferase superfamily}

Owing to the central role of the PCP domain in shuttling intermediates to the different catalytic domains during peptide assembly, NRPS enzymes are inactive until converted to their holo form by a partner PPTase. The PPTases were characterised as a distinct superfamily in a landmark paper by Lambalot et al (1996). Since then interest in PPTases has increased owing to multiple applications in biotechnology including for site-specific labelling and detection of biosynthetic gene clusters from metagenomic libraries, and also their potential to provide a novel drug target in both bacteria and fungi. This section will review the structure and function of PPTases and the biotechnological applications of PPTases.

\subsubsection{PPTases post-translationally modify carrier proteins}

PPTases are an enzyme superfamily that is represented in all known prokaryotes, eukaryotes and archaea (Beld et al., 2014). The post-translational attachment of a Ppant prosthetic group to carrier protein (CP) domains is essential for the functionality of large modular enzymes involved in both primary (e.g., fatty acid synthetases) and secondary biosynthesis (e.g., non-ribosomal peptide synthetases and polyketide synthetases). 
PPTases can broadly be characterised into three distinct groups based on conserved sequence motifs and structural features (Beld et al., 2014).

\subsubsection{Type I PPTases}

Type I PPTases are bacterial transferases that are approximately 120 residues in size and typically activate fatty acid synthase (FAS) systems. This class is exemplified by acyl carrier protein synthase (AcpS) from E. coli, which was discovered in the 1960s (Alberts and Vagelos, 1966). AcpS activates the Type II fatty acid synthetase (FAS) acyl carrier protein (ACP) and has a relatively narrow substrate specificity when compared to Type II PPTases. However, it will still modify some PCP domains and accept CoA analogues (Gehring et al., 1997). Several different AcpS-type PPTases have been characterised, with the first published structure being AcpS from Streptococcus pneumoniae (Beld et al., 2014). S. pneumoniae AcpS has a trimeric quaternary structure and the active site is located in the cleft between the different monomers, which results in AcpS type PPTases having three active sites. Other AcpS type PPTases have also been crystallised and found to exhibit the same broad structural features, including AcpS from M. tuberculosis (Dym et al., 2009) and AcpS from B. subtilis (Parris et al., 2000).

\subsubsection{Type II PPTases}

Type II PPTases have a much broader substrate specificity than Type I PPTases and typically activate the PCP domains of NRPS and the ACP domains of polyketide synthases (PKS). They are often found within or in close proximity to the biosynthetic gene cluster they are responsible for activating (Beld et al., 2014).

Sfp from B. subtilis is the most well studied and characterised PPTase. The crystal structure of $\mathrm{Sfp}$ has been solved at $1.8 \AA$ resolution and exhibits a pseudo-dimeric fold which is comprised of two similar sub-domains connected by a short polypeptide loop (Reuter et al., 1999). Unlike the AcpS type PPTases it only contains a single active site, which requires $\mathrm{Mg}^{2+}$ to be bound for catalytic activity (Reuter et al., 1999). In addition to Sfp, the human PPTase (Hs-Ppt) and the M. tuberculosis PPTase PptT have also had their structures elucidated. Hs-Ppt is an interesting case as the structure is the same as a Type II PPTase, however its primary biological role is to modify the ACP from Type I FAS 
(Bunkoczi et al., 2007). This role is usually filled by AcpS type PPTases in bacteria. The structure and function of PptT is discussed in detail in Section 3.2.1 and Section 3.2.2

\subsubsection{Type III PPTases}

Type III PPTases have been identified in fungi and are the least well studied group of PPTases. Unlike Type I and Type II PPTases they are fused to the C-terminus of the yeast FAS-1 system (Beld et al., 2014). Intriguingly, Type III PPTases appear in multiple states depending on the quaternary structure of the FAS complex. These states are either a monomer domain outside of the main FAS structure, or as a functional dimer when the FAS complex is fully formed, or, if it is cleaved from the FAS complex to yield a separate protein, as a functional trimer (Beld et al., 2014).

\subsection{PPTase inhibition as a novel drug target}

The essential nature of PPTases has led to attention been focused on probing their potential usefulness as a drug target. PptT from M. tuberculosis has been examined indepth as potential drug target, and this work is discussed in detail in Section 3.2.1 along with similar research investigating PcpS from $P$. aeruginosa. Several compounds have been identified that inhibit these Sfp-type PPTases. Inhibitors of AcpS-type PPTases have also been identified, and this work is discussed in detail in Section 4.1.3. While several papers have reported on the effectiveness of PPTase inhibition in vitro, only two have reported the results of testing the identified inhibitors in vivo for efficacy. These two papers are profiled below.

Sfp from B. subtilis was screened by Foley et al (2014) for inhibitors from the Molecular Libraries Small Molecule Repository, which contains 311,260 compounds. Screening was conducted at eight concentrations, ranging from $3.6 \mathrm{nM}$ to $114 \mu \mathrm{M}$, and the methodological basis for the screen is discussed in more detail in Section 1.7. In this study, 338 structurally diverse compounds were identified for follow up, which included profiling their activity against AcpS and testing their activity against an engineered strain of $B$. subtilis that depends solely on Sfp for viability. From this group of compounds, they identified a compound ("compound 1"), a reversible non-competitive inhibitor that has 
inhibitory activity against $\mathrm{Sfp}, \mathrm{AcpS}$ and mild antimicrobial activity against $B$. subtilis (Foley et al., 2014).

Structure activity relationship studies were completed on compound 1 to improve activity with Sfp and AcpS, as well as its general antimicrobial activity. In addition, the compounds were profiled for toxicity with human cells and inhibition of the human PPTase. These assays led to the development of ML267 which had an IC $\mathrm{C}_{50}$ (i.e., the concentration when $50 \%$ inhibition occurs relative to an uninhibited control) of $290 \mathrm{nM}$ with Sfp and $8.1 \mu \mathrm{M}$ with AcpS. Importantly, no inhibition of the human PPTase was observed up to a concentration of $125 \mu \mathrm{M}$, as this could potentially be a key source of toxicity to patients (Foley et al., 2014).

ML267 was then assayed for activity against a broad range of bacteria and fungi and was found to only be inhibitory against Gram-positive bacterial species. Foley et al speculated that this was due to Gram negative and fungal efflux pump activity rather than structural differences in PPTases from the different bacterial and fungal strains. This was confirmed when an E. coli mutant lacking the AcrAB-TolC efflux pump system was found to be highly susceptible to compound 55. Currently ML267 represents the first known PPTase inhibitor to have an anti-microbial effect in vivo (Foley et al., 2014). A recently published paper looked at the inhibition of PptA from Aspergillus fumigatus. PptA activates NRPSs and PKSs involved in siderophore and lysine biosynthesis, and is essential for pathogenicity of the fungus. In a $\Delta p p t A$ mutant the lysine and iron requirements exceeded the levels available in mammalian tissues and rendered the strain avirulent in insect and murine infections (Johns et al., 2017).

A fluorescence polarisation assay was used to identify inhibitors of PptA from a small molecule library consisting of 6,231 compounds. From this library, three PPTase inhibitors were identified: PD 404,182, 6 nitroso-1,2-benzopyrone (6-NOBP) and calmidazolium chloride (all of which had previously been identified as inhibitors of other PPTases) (Yasgar et al., 2010). Interestingly 6-NOBP had a higher $\mathrm{IC}_{50}$ of $35.2 \mu \mathrm{M}$ against PptA, compared to its activity against previously tested bacterial PPTases (Johns et al., 2017). 
The three PptA inhibitors were all found to be effective inhibitors of fungal growth. To determine if the inhibition of PptA was the direct cause of the anti-fungal activity, the growth media of $A$. fumigatus was supplemented with both lysine and iron. Only for 6NOBP was a reversal of activity observed upon supplementation, with the anti-fungal activity being reduced 2 -fold against $A$. fumigatus and four-fold against Aspergillus flavus, another fungal species that was also tested. These results indicate that PptA can indeed be targeted in vivo and inhibitors identified in a selective manner (Johns et al., 2017).

While the two papers profiled above have shown that PPTases can be an effective drug target, some debate exists on exactly how effective they are. A study by Brinster et al showed that the FAS-II pathway in Gram positive bacteria is an ineffective target for antimicrobial activity as Streptococcus agalactiae, an opportunist pathogen, can scavenge fatty acids from serum and hence its endogenous FAS-II is not essential for in vivo growth (Brinster et al., 2009). Moreover, even when a PPTase has been shown to be essential for pathogenesis, it is still unknown exactly what level of inhibition is required for a bactericidal effect in most species. It has been shown that at least $95 \%$ inhibition is required for the Type II PPTase in M. bovis to inhibit growth in vitro (Leblanc et al., 2012). Despite the lack of characterisation of PPTase inhibitors in vivo, they appear to be worth exploring further as a drug target. In addition to those implicated in primary metabolism, Type II PPTases have also been shown to be essential for controlling the production of secondary metabolites including virulence factors (Cheng et al., 2016; Márquez-Fernández et al., 2007). For example, the scavenging of iron by siderophores is becoming an attractive drug target in M. tuberculosis (Lamb, 2015) and PptT plays an essential role in this pathway.

\subsection{Detection of PPTase inhibition}

Several different screening techniques have been developed for the detection of PPTase inhibition, the majority of which have focused on the prototypical PPTase, Sfp from $B$. subtilis. Screens against the second most studied Type II PPTase target, M. tuberculosis PptT, are discussed in detail in Section 3.2.3. 
Initial attempts to develop PPTase inhibitor screens focused solely on AcpS inhibitors, and these screens are discussed in more depth in Section 4.1.3. The first screen for a Type II PPTase inhibitor was developed by Foley et al (2009), who used a Förster resonance energy transfer (FRET) assay. A modified acceptor peptide (in this case a peptide called YbbR that was known to be a good substrate for Sfp) was designed to generate a FRET pair upon conversion to the crypto product when the PPTase tethers the Ppant analogue rhodamine-mCoA to it, resulting in a quenched signal in a microplate assay. Foley et al tested a panel of known AcpS inhibitors and found that none of them had inhibitory activity with Sfp. This screen represented the first Sfp type screen amenable to high throughput screening (HTS) (Foley et al., 2009).

A follow up paper from the same lab group by Yasgar et al (2010) improved on the FRET based screen use to identify Sfp inhibitors. This paper represented the first attempt to identify small molecule inhibitors of Sfp from chemical libraries. While the FRET assay described in the previous paragraph worked well in a 96 well plate format, scaling it up to a 1536 well format resulted in significantly reduced performance. To overcome this issue the assay was redesigned to use a Black Hole Quencher-2 labelled YbbR peptide, which better matched the emission spectrum of rhodamine-mCoA, resulting in improved performance. The assay was miniaturised into a $4 \mu \mathrm{L}$ volume and screened in 1536 well microtitre plates. Using this screen, they interrogated the LOPAC ${ }^{1280}$ library and identified the first Sfp inhibitors including SCH-202676 and 6-NOBP (Yasgar et al., 2010).

Another paper also published by Duckworth et al (2010) described a second HTS method to detect inhibitors of Sfp, using a fluorescence polarisation (FP) assay. Since Sfp can accept a variety of different fluorescent CoA analogues, they had a wide choice of probes available for use. They settled on BODIPY-labelled CoA as it had previously been used in HTS. When Sfp binds the BODIPY-labelled CoA and is excited with polarising light, the Sfp-labelled CoA complex doesn't tumble in solution before reemitting a photon and remains polarised. If an inhibitor blocks the binding of the labelled CoA, the CoA will tumble rapidly due to its smaller molecular weight. Since the molecule is tumbling rapidly the emitted light is largely depolarised (Duckworth and Aldrich, 2010). Several compounds previously identified by Yasgar et al as Sfp inhibitors were tested to see if they were competitive with respect to CoA. Four compounds were tested and only one 
was found to be weakly competitive, however the authors didn't state exactly which compounds were tested (Duckworth and Aldrich, 2010).

Kosa et al (2014) modified the screening process developed by Foley et al and Yasgar et al after finding they were unable to detect signal quenching by Hs-Ppt using the FRET based system. They speculated that this could be due to the Hs-Ppt not recognising the YbbR peptide. Instead they decided to use the VibB CP and to develop a FP assay that would use the difference in tumbling rates to determine if the rhodamine-CoA had bound to this CP. This method also allowed them to easily modify the carrier protein being screened against as well as the PPTase, which facilitated the subsequent use of human ACP to detect inhibition of the Hs-Ppt (although when this second system was implemented the assay $Z$ scores were much less robust) (Kosa et al., 2014). They then optimised and expanded their VibB-based FP assay for high throughput screening in 1536 well plates and screened $\sim 310,000$ compounds from which approximately 11,000 compounds with some level of inhibitory activity were detected (Foley et al., 2014; Kosa et al., 2014) . The quantitative HTS results for the assay are deposited in the PubChem BioAssay Database with the bioassay record of AID 1490, and a summary available under the record number AID 1819.

\subsection{Site specific labelling using PPTases}

In addition to offering promise as drug targets, PPTases have been employed in diverse functional applications for biotechnology. Since Sfp is able to readily accept CoA analogues, a feature that underpinned the different inhibitor screening applications described in Section 1.7, it is also an excellent tool for site specific labelling. This broad specificity has been exploited for the attachment of different CoA analogues to carrier proteins to allow for western blot identification and affinity purification (La Clair et al., 2004). It has also allowed the site-specific labelling of recombinant proteins into which entire or partial CP domain sequences have been embedded.

Although carrier protein domains are relatively small, they are still frequently too large to be an effective protein tag without interfering with protein function. To overcome this limitation, several different PPTase substrate tags have been developed. The 11 
residue YbbR tag was identified from a genomic library of $B$. subtilis using phage display as a substrate for the binding of small molecule-CoA conjugates (Yin et al., 2005). The initial phage display experiments identified a range of truncated proteins to which a CoA derivative had been attached. These proteins were truncated to identify the minimal binding sequences until a top candidate sequence, the YbbR tag, had been developed. The YbbR tag is 11 residues in length and adopts an $\alpha$-helix conformation in solution (Yin et al., 2005), which is in direct contrast to the AcpS-substrate A-4 tag discussed later in this section. The $\alpha$-helix conformation appears to be important for the binding of Sfp, as tags that didn't form a helix were not recognised by Sfp (Yin et al., 2005).

The peptide tags used in site specific labelling by PPTases were further refined by Zhou et al (2007) who used a phage-display library to identify peptide tags that could be selectively bound by either Sfp or AcpS (these tags being modified variants derived from a parental YbbR tag). From this work two tags were developed, S6 and A1. The catalytic efficiency of Sfp with the $\mathrm{S} 6$ tag was 442 -fold higher than that exhibited by AcpS. In contrast, AcpS exhibited 30-fold higher catalytic efficiency over Sfp with the A1 tag (Zhou et al., 2007), indicating that the dual PPTase/tag system could be used as a selective labelling tool. This would allow the tagging of two different proteins in a complex mixture at the same time (Zhou et al., 2007).

In a proof of concept experiment, the S6 tag was fused to a cell surface receptor for epidermal growth factor, while the A1 tag was fused to the cell surface receptor transferrin receptor 1 . Sfp and AcpS were both then used to transfer Texas red-CoA to the receptors that then could be visualised. In addition, both receptors were exposed to their ligands with an Alexa 488 antibody attached. In each case, direct visualisation of native ligand binding indicated that the tags were not interfering with the receptor function (Zhou et al., 2007).

Further work by Zhou et al improved the A1 tag and shortened it from $12 \mathrm{AA}$ to a minimal 8 AA tag called the A-4 tag, using NMR titration experiments. Interestingly, Zhou et al found that no secondary structure was apparent in the shortened tag, indicating that the alpha helix structure isn't essential for binding by AcpS (Zhou et al., 2008), which is in direct contrast to the earlier observations with the YbbR-derived peptides and Sfp (Zhou et al., 2007). 
Sunbul et al (2009) used phage display libraries to modify the substrate specificity of Sfp from biotin-conjugated coenzyme A to $3^{\prime}$ dephospho CoA as a substrate. The top mutant (R4-4) had a 300-fold increase in catalytic activity (Sunbul et al., 2009) with deophospho CoA. R4-4 also had an increased catalytic efficiency over Sfp with CoA (Sunbul et al., 2009). This paper shows that Sfp can be readily evolved to recognise different CoA analogues.

\subsection{Utility of PPTases for identifying biosynthetic gene clusters}

Another application of PPTases in biotechnology has been in screening for natural product gene clusters from genomic or metagenomic DNA libraries. A large proportion of the drugs available on the market are natural products, many of which are derived from secondary metabolites synthesised by NRPSs and PKSs (Newman and Cragg, 2016). Traditionally microbes that have been culturable in the laboratory setting have been the source of many drugs, however only a small fraction of known microbes are culturable in a lab setting (Stewart, 2012). By targeting the genomes of microbes in order to identify new natural products and the biosynthetic gene clusters that specify them, it is possible to bypass culturing the organisms in the laboratory. This technique is known as metagenomic screening (Simon and Daniel, 2011).

The first utilisation of PPTases in metagenomic screening was described by Yin et al in 2007. They utilised a combination of phage display and Sfp to recover NRPS and PKS CP domains using biotin-CoA. A shotgun library generated from the genomes of $B$. subtilis and $M$. xanthus was displayed on phages, and phages that acquired genetic fragments encoding CPs were captured when biotin-CoA was bound to them by Sfp (Yin et al., 2007). The proof of concept study used B. subtilis as the genome was available to cross reference known CP and ACP domains. After five rounds of enrichment, $85 \%$ of the selected clones contained either a CP or ACP domain, while the other $15 \%$ of genomic fragments contained in-frame stop codons indicating that they were being enriched due to non-specific binding to the streptavidin surface. Out of the 100 clones that Yin et al sequenced, 17 of the 39 total PCPs/ACPs that had previously been identified as being 
encoded by NRPS and PKS genes within the $B$. subtilis genome were present. To then determine if Sfp could be used to profile an alternative bacterial species $M$. xanthus was used, as its genome had recently been sequenced. In contrast to the B. subtilis screen, only 22 carrier proteins from the 119 CPs annotated in the genome were recovered. It was concluded that this was likely a consequence of Sfp not recognising the majority of M. xanthus CPs (Yin et al., 2007).

The same technique was used to profile a soil metagenomic library in 2009, with Sfp again used to pull out CP and ACP domains (Zhang et al., 2009). From this library, a range of novel CP and ACP domains were identified. The researchers noted that by using a PPTase with a broader specificity or a series of different PPTases it should be possible to identify more CP domains, as this method relies explicitly on Sfp recognising the domain. However, a key benefit of this method is it doesn't require the protein to be functional or to be heterologously expressed in high levels to be identified in the screen, which should facilitate the identification of biosynthetic gene clusters (Zhang et al., 2009).

An alternative method that utilises PPTases for metagenomic library screening relies on PPTases acting as a molecular beacon, based on the observation that PPTase genes are often located within biosynthetic gene clusters. This approach was first discussed by Mootz et al (2002) who used a conditionally lethal Lys5 PPTase deletion strain of S. cerevisiae to screen for complementing PPTases. Lys5 is the PPTase responsible for posttranslationally modifying the PCP domain of Lys2, an enzyme critical to the synthesis of lysine in S. cerevisiae. An uncomplemented $\Delta / y s 5$ mutant is only viable if the growth medium is supplemented with lysine (Mootz et al., 2002). Mootz et al complemented the $\Delta / y s 5$ mutant with a range of different cloned PPTases, which rescued its ability to grow in lysine deficient media. This result indicated that the system might be applicable as a screening tool to identify new PPTases from DNA libraries, and thereby recover fragments of biosynthetic gene clusters (Mootz et al., 2002).

The concept was first put into practice by the Ackerley lab who used a gene encoding a single-module blue-pigment-synthesising NRPS as a screening tool to detect cloned PPTase genes from a soil metagenome library via induction of blue pigment synthesis (Owen et al., 2012). This functional metagenomic screen is discussed in more detail in Section 1.11.2. An alternative approach was published in 2013 by Charlop-Powers et al, 
who selected for PPTases from metagenomic library fragments that restored siderophore biosynthesis in a strain of $E$. coli in which the endogenous Type II PPTase gene entD had been deleted (Charlop-Powers et al., 2013). E. coli is still viable without this PPTase however it lacks the ability to produce the iron scavenging siderophore enterobactin, meaning that it cannot grow in iron restricted conditions. Charlop-Powers et al reasoned that a PPTase expressed from a metagenomic fragment that was able to post translationally modify the enterobactin pathway CPs would render the $E$. coli $\Delta e n t D$ strain capable of growth on iron limited media. This selection allows large metagenomic libraries to be screened for PPTases and their associated bioactive gene clusters, and in a pilot study they were able to enrich the library for bioactive gene clusters. One hundred clones from the enriched library and 100 random clones from the parent library were sequenced. The enriched library had $17 \%$ of the sequence space devoted to NRPS/PKS genes. This is in contrast to the unenriched clones which only had $0.45 \%$ devoted to NRPS/PKS genes (Charlop-Powers et al., 2013).

\subsection{Blue Pigment Synthetase A}

This thesis focuses on the NRPS Blue Pigment Synthetase A (BpsA), a single module NRPS that produces the vibrant blue pigment indigoidine (Fig. 1.2B) (Takahashi et al., 2007). Indigoidine was first identified in the bacterium Pseudomonas indigofera in the 1930s (Elazari-Volcani, 1939), however it wasn't until the 1960s that indigoidine was isolated and purified from three members of the genus Erwinia (Starr et al., 1966). In the early 2000s the gene cluster responsible for the synthesis of indigoidine were identified in the plant pathogen Erwinia chrysanthemi (Reverchon et al., 2002). Using transposon library mutagenesis screening, Reverchon et al identified three open reading frames; ind $A$, ind $B$ and ind $C$ (the latter being a bpsA homolog). IndC was determined to contain an $A$ domain that has an integrated oxidation domain in the $\mathrm{C}$ terminal region, a PCP domain and TE domain). Through sequence analysis they identified the Stachelhaus coding residues and determined that the A domain recognised L-glutamine (Reverchon et al., 2002).

In E. chrysanthemi indigoidine production is crucial for pathogenesis. Reverchon et al speculated, based on the chemical structure (Fig. 1.2C), that indigoidine might be a 
radical scavenger. Evidence for this hypothesis was provided by the observation that when indigoidine was exposed to hydroxyl radicals it discoloured. This hypothesis was strengthened when it was observed that mutants having increased expression of the ind $C$ locus had increased tolerance to oxidative stress (Reverchon et al., 2002).

\subsubsection{Structure and function}

A standalone bpsA gene (i.e. not associated with homologues of indA or ind $B$ ) was discovered and characterised by Takahashi et al (2007). They identified a biosynthetic gene that they named bpsA from the plant pathogen Streptomyces lavendulae serendipitously, while trying to find the gene cluster of another NRPS (Takahashi et al., 2007). The bpsA gene was used to transform E. coli along with the gene encoding the PPTase Svp to activate it, as it was found that the native E. coli type II PPTase EntD is relatively inactive with BpsA. In contrast, the $b p s A^{+} s v p^{+}$strain produced a deep blue pigment, which mass spectrometry confirmed to be indigoidine. BpsA has the same domain architecture as IndC, i.e. an interrupted A-OX-A-T-TE structure (Fig. 1.2A). Takahashi et al expressed bpsA with a $6 \mathrm{His}$ tag and purified the recombinant protein, which allowed them to confirm that L-glutamine was the only amino acid accepted by the $A$ domain using ATP/PP $\mathrm{i}_{\mathrm{i}}$ assays. They also demonstrated that FMN was bound in the oxidation domain. BpsA is capable of producing indigoidine in vitro (Fig. 1.2B) which indicates that the other genes in the IndC and BpsA operon aren't essential for the synthesis of indigoidine (Takahashi et al., 2007).

Takahashi et al also undertook a mutational analysis study on the oxidation domain and found that when the oxidation domain was mutated so it was unable to bind FMN, indigoidine synthesis was interrupted, and in addition the rate of adenylation activity was $70 \%$ slower. Intriguingly, if the oxidation domain is separately purified and added to the mutated (non FMN binding) BpsA, in vitro synthesis of indigoidine is restored (Takahashi et al., 2007).

Indigoidine appears to have different biochemical roles depending on the species of bacterium that is synthesising it. In both E. chrysanthemi and S. lavendulae it appears to protect the bacterium from oxidative stress (Reverchon et al., 2002; Takahashi et al., 2007). In other bacteria, it appears to have an anti-microbial effect. Bacteria from the 
Roseobacter clade appear to produce indigoidine to inhibit the colonisation of Vibrio fischeri. Interestingly it appears that the anti-microbial activity is mediated by the reduced, colourless leuco form of indigoidine (Cude et al., 2012; Gromek et al., 2016). The anti-microbial effects of indigoidine are discussed in more detail in Chapter Six.

A

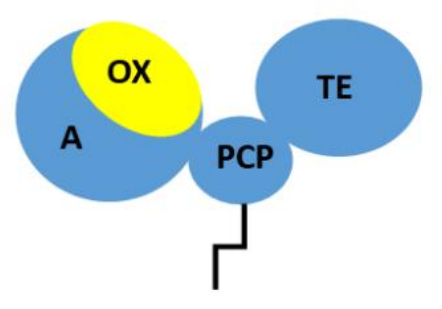

C<smiles>NC1=CC(=C2C=C(N)C(=O)NC2=O)C(=O)NC1=O</smiles>

B

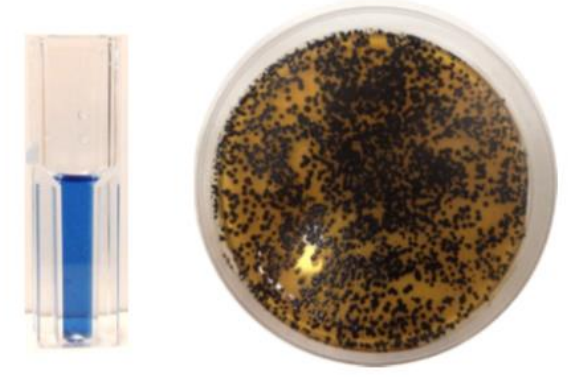

Figure 1.2 BpsA is responsible for indigoidine synthesis: (A) Schematic diagram of BpsA, showing the $A$ domain, $O X$ domain, PCP domain with Ppant arm attached, and TE domain. (B) Indigoidine is detectable in vitro (resuspended in DMSO) and in vivo. (C) The chemical structure of indigoidine.

\subsection{Biotechnological uses of BpsA and indigoidine}

Because of the simple nature of BpsA and the ease in which it can produce a pigment both in vivo and in vitro, a variety of different biotechnological applications have been explored. These include several applications that were developed or advanced in the research described in this thesis, i.e. detection of PPTase inhibitors using native coenzyme A (Chapter 3 and 4), detection and quantification of L-glutamine and ATP 
(Chapter 5 and 6), and production of novel indigoidine analogues (Chapter 6). Published applications not discussed in detail in later chapters are described here.

\subsubsection{Production of indigoidine in E. coli}

Indigoidine has attracted interest as a commercial dye as natural colourants are frequently preferable over artificial dyes (Downham and Collins, 2000). Two papers have been published that focus on increasing the level of indigoidine produced in E. coli. In one of these, Yu et al identified a gene cluster in the indigoidine producing bacterium $S$. chromofuscus ATCC49982 that contained five open reading frames including an unusual gene they designated ind $B$. The ind $C$ ( $a$ bps $A$ homolog) gene from this cluster was cloned into an E. coli producer strain BAP1, and after a period of optimisation they achieved a yield of $2.78 \mathrm{~g} / \mathrm{L}$. Intriguingly, when they then co-expressed IndC with IndB, another gene present in the gene cluster, the indigoidine yield was increased by more than $40 \%$, to $3.93 \mathrm{~g} / \mathrm{L}$. IndB is 614 residues in size and the $\mathrm{N}$ terminus appears to be a phosphatase found in other indigoidine producing gene clusters while the $\mathrm{C}$ terminus aligns to a hypothetical protein also identified in gene indigoidine gene clusters (Yu et al., 2013).

In a 2015 paper the same lab group further improved the indigoidine yield in $E$. coli by modifying the $E$. coli strain and fermentation broth. The glutamine synthetase gene $(g \ln A)$ was co-expressed and nitrogen containing salts were added to the fermentation broth. These modifications resulted in a yield of $7.1 \mathrm{~g} / \mathrm{L}$ of indigoidine (Xu et al., 2015). This work showed that it should be possible to produce high yields of indigoidine from $E$. coli if it ever becomes a commercially attractive product.

\subsubsection{As a multi-kingdom reporter gene}

Work by Müller et al (2012) has shown that BpsA can be expressed in mammalian cells with minimal effort. The $b p s A$ and the PPTase svp genes were codon-optimised for expression in human cells. The constructs were then transfected into human embryonic kidney (HEK-293) cells. In contrast to E. coli indigoidine in HEK-293 is cell entrapped, which suggests mammalian cells lack the ability to transport indigoidine out of the cell, and instead it appears to be stored in granules within the cytoplasm. The cells expressing BpsA can be quantified by flow cytometric analysis and show increased cell 
volume and granularity compared to control cells not producing the blue pigment (Müller et al., 2012). This work was the first time a bacterial NRPS had been expressed in mammalian cells and paves the way for novel future treatments that may use nonribosomal peptides, for example engineering immune system cells to synthesise antibacterial peptides.

A technique similar to lacZ $\alpha$ based blue/white screening has also been developed that uses BpsA as a reporter (Xie et al., 2017). One advantage of this technique is that it doesn't require an extracellular chromogenic substrate (as opposed to the requirement for X-gal for lacZa screening). Xie et al used a homolog of bpsA called idgs from Streptomyces and $s f p$ to develop a vector capable of blue/white screening. The idgS gene cassette was inserted into a vector along with $s f p$ harbouring an in-frame insertion of a multiple cloning site (MCS) close to the $\mathrm{N}$-terminal. This allowed the selection of white colonies which had the gene cloned into the MCS (Xie et al., 2017). While this is a useful system, indigoidine has been observed to have mildly toxic effect on $E$. coli cells synthesising it (Brown et al., 2017), which may limit the usefulness of this screen.

\subsubsection{Screening of metagenomic libraries}

As noted in Section 1.9, BpsA was developed in our laboratory as a functional screening tool to identify novel PPTases from metagenomic libraries. To improve the diversity of PPTases that were discoverable, PCP domains derived from other bacterial species (from the second module of $p v d D$ from $P$. aeruginosa and the PCP domain from entF from $E$. coli) were inserted in replacement of the endogenous PCP domain (Owen et al, 2012; Owen et al, 2016). To improve activity in the recombinant BpsA variants expressing the foreign carrier proteins, directed evolution via error-prone PCR was used (Owen et al., 2016).

The BpsA variants were expressed in a strain of $E$. coli with the native entD knocked out. This strain was incapable of activating any of the BpsA mutants as E. coli AcpS is unable to post-translationally modify BpsA. Two small-insert soil metagenome libraries were screened using the BpsA reporter variants. From these, a range of novel PPTases were identified, several of which were only active with one or two of the three bpsA reporter constructs that were employed. In addition to the novel PPTase genes, several open 
reading frames (ORFs) that aligned to NRPS and PKS domains were also identified, which indicated that this method could be used to recover biosynthetic gene clusters along with the PPTases (Owen et al., 2012).

This same approach has also been adapted for use in Streptomyces albus, a well-known secondary metabolite producer strain (Baltz, 2010). An endogenous PPTase knockout mutant was created and wild type bpsA was used as a reporter. A soil eDNA cosmid library was screened in this strain, and several novel NRPS, PKS and NRPS/PKS hybrid gene clusters were identified. While this technique is the same one that was pioneered in our laboratory, by screening in a strain of bacteria that is better able than $E$. coli to express natural products, Bitok et al were able to identify more novel gene clusters, and furthermore they used cosmids which facilitated the recovery of whole genes rather than a small insert library (Bitok et al., 2017).

\subsubsection{Profiling of PPTase activity}

Owen et al (2011) developed a range of rapid and flexible assays that used BpsA as a reporter for PPTase activity, and noted that one application could be to screen chemical libraries to detect PPTase inhibitors. Chapter Three of this thesis describes the first implementation of this concept. In another application, Owen et al showed that by continuously monitoring the rate of indigoidine synthesis as a PPTase converts BpsA from the inactive apo form to the active holo form, it is possible to determine the Michaelis-Menten kinetic parameters of a PPTase in respect to varying concentrations of either CoA or BpsA (Fig 1.3) (Owen et al., 2011). Owing to the unusual absorbance profiles characteristic of indigoidine synthesis in an aqueous solution (Fig. 1.3A), kinetics are derived from the maximal rates of indigoidine synthesis (Fig. 1.3B) rather than an endpoint measurement of indigoidine concentration. These assays have also been used by additional lab groups including for measuring the activity of PPTases from species of Mycobacterium (Vickery et al., 2014). 

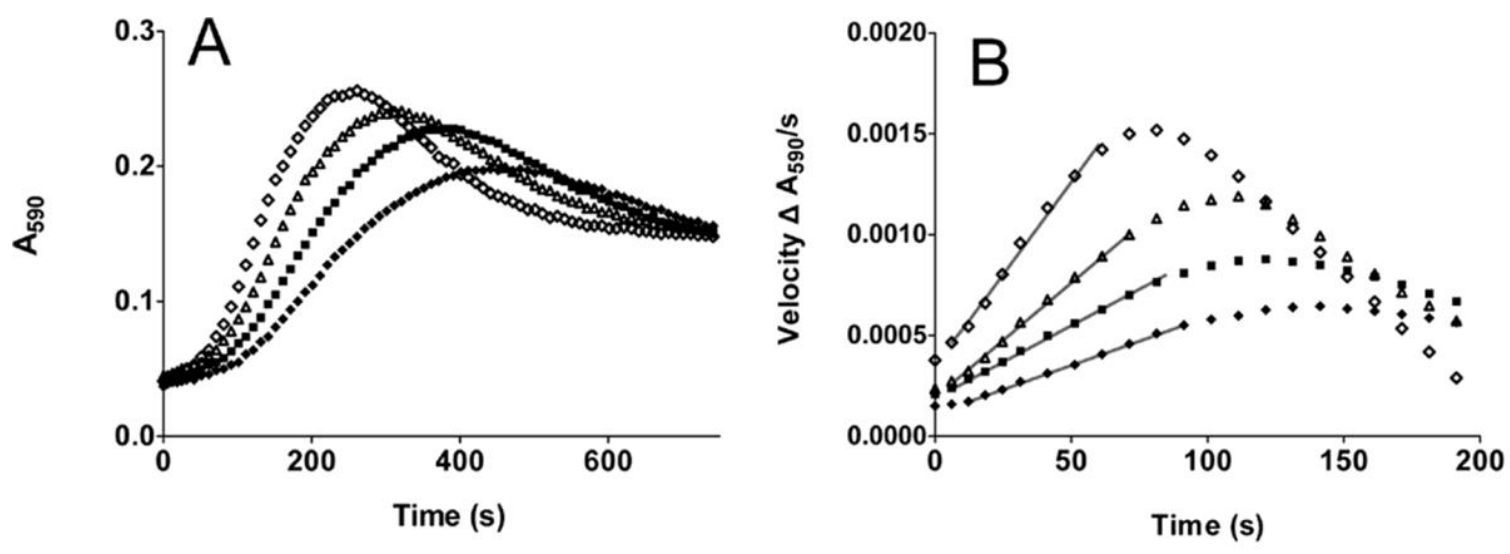

Figure 1.3 Deriving PPTase kinetic parameters using BpsA as a reporter: (A) Raw A590 values for indigoidine synthesis as BpsA is progressively converted from the apo form into the holo form by PcPS. The reaction velocity increases as the concentration of COA is increased. Indigoidine syntheis has a characteristic curve of a rapid increase then decrease in $A 590$ values as it drops out of solution. The diffferent concentrations of CoA are $3.1(\diamond) 1.56(\square) 0.78(\Delta)$ or $0.39(\Delta) \mu M C O A(B)$ The velocity of PPTases can be derived by measuring the linear region of the veloctiy against time data derived from panel A. Figure is adapted with permission from (Owen et al., 2011).

\subsubsection{Interrogation of PCP domain activity}

Owen et al (2011) also developed a competition assay to probe the activity of PPTases with different CPs. In this assay, BpsA and purified CP domains compete for a limited amount of COA. The rate of indigoidine synthesis then acts as a reporter for PPTase specificity towards different CPs (Owen et al., 2011). This technique has again been adopted by other laboratory groups and was used to help determine the specificity of PptT toward AcpM from M. tuberculosis (Zimhony et al., 2015).

In addition to interrogating carrier proteins in situ, BpsA has also been used to probe CP domain activity within the enzyme itself. To achieve this, Beer et al (2013) developed a range of modified forms of the BPSA PCP domain order to identify mutations that improved overall function (Beer et al., 2014). Subsequent work by Owen et al also showed that a range of alternative PCP domains could be substituted into BpsA and rendered functional via only a few point mutations (Owen et al., 2016). Each of these papers is discussed in more depth in Section 4.1.5. 


\subsection{Research Aims}

The overall goal of the research described in this thesis was to apply BpsA to achieve useful biotechnological outcomes. The first aim of this work was to develop and optimise a screen to detect inhibitors of the essential PPTases PptT and PcpS from the clinically important bacterial strains $M$. tuberculosis and $P$. aeruginosa. Both of these enzymes are type II PPTases that have relatively broad substrate specificity. The optimised screen was then used to interrogate three commercially available drug libraries to identify and characterise PPTase inhibitors.

The second aim of this thesis was to evolve a modified form of BpsA that was capable of being post-translationally modified by AcpS from M. tuberculosis, a type I PPTase that has a narrow substrate specificity. To achieve this, two rational constructs were created by substituting the second alpha helix from M. tuberculosis AcpM or FAS-1 into the equivalent location of BpsA. Error prone PCR was then applied in an attempt to generate functional variants that might have utility in screening chemical libraries to identify $M$. tuberculosis AcpS inhibitors.

The third aim of this project was to develop a commercially applicable assay to detect the concentration of L-glutamine present in a range of biologically relevant conditions, by using holo-BpsA to synthesise indigoidine from the glutamine. By quantifying the amount of indigoidine synthesised it was reasoned that it should be possible to accurately measure the amount of glutamine originally present.

Based on the findings from addressing aim three it might be possible to use BpsA to quantify ATP levels in a range of complex media. Aim four therefore became to further expand the uses of the BpsA biosensor, including the detection of the adenylation reaction by $A$ domains.

Finally, the literature had shown that A domains, while specific can sometimes accept other monomers, especially similar analogues. Thus, the last aim of the thesis was to determine if indigoidine-like compounds could be synthesised from L-glutamine analogues, such as alanyl-glutamine, an important cell culture additive. If successful, this would improve the usefulness of the biosensor. 


\section{Chapter 2- Materials and methods}

\subsection{General chemical reagents and materials}

Chemicals, reagents and media used in this study were obtained from either SigmaAldrich (St Louis, MO, USA) or Thermo Fisher Scientific (Waltham, MA, USA), unless otherwise stated. L-glutamine for use in media preparations was purchased from iHerb (Perris, CA, USA). Restriction enzymes were purchased from New England Biolabs (NEB; Ipswich, MA, USA) or Thermo Fisher Scientific (Waltham, MA, USA). IPTG (isopropyl $\beta$-Dthiogalactoside) and BioMix ${ }^{\mathrm{TM}}$ Red Polymerase Mastermix were supplied by Bioline (London, UK). Phusion ${ }^{\mathrm{TM}}$ high-fidelity DNA polymerase was supplied by Finnzymes (Espoo, Finland). T4 DNA ligase was supplied by either Bioline or Invitrogen (Carlsbad, CA, USA). Mutazyme was obtained from Agilent Technologies (Santa Clara, CA, USA). The LOPAC $^{1280}$ and Spectrum Collection were both kindly provided by the Victoria University of Wellington Chemical Genetics Laboratory. The NIH library diversity Set II was obtained from the NIH Developmental therapeutics programme (Rockville MD, USA).

\subsection{Bacterial strains and plasmids}

\subsubsection{Bacterial strains}

The bacterial strains used in this study are listed in Table 2.1

\begin{tabular}{|c|c|c|}
\hline Strain & Relevant characteristics & Source \\
\hline DH5 $\alpha \lambda$ pir & 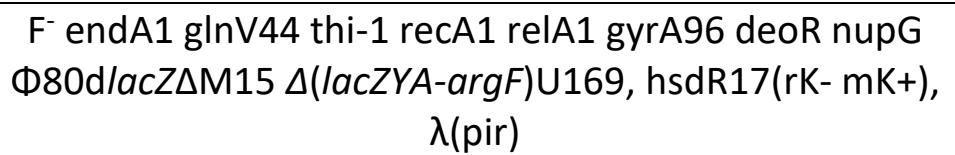 & Invitrogen \\
\hline BL21 & $\mathrm{F}^{-}$ompT gal dem lon $\mathrm{hsdS}_{B}\left(\mathrm{r}_{B}^{-} \mathrm{m}_{B^{-}}\right) \lambda(\mathrm{DE} 3)$ & Novagen \\
\hline BL21 $\Delta$ entD & $\mathrm{BL} 21 \Delta$ entD & Owen 2012 \\
\hline PA01 & P. aeruginosa wildtype lab strain & Lab stock \\
\hline
\end{tabular}

Table 2.1 Bacterial strains used in study: Abbreviations for $E$. coli strains relevant characteristics are available at: http://www.openwetware.org/wiki/E._coli_genotypes\#Nomenclature_.26_Abbreviations 


\subsubsection{Plasmids}

\begin{tabular}{|c|c|c|}
\hline Plasmid & Relevant characteristics & Source \\
\hline pET-28a(+) & $\begin{array}{c}\text { Kan }^{\mathrm{R}} \text {, expression vector for His-tagged enzyme } \\
\text { purification, T7 promoter, ColE1 origin of } \\
\text { replication }\end{array}$ & Novagen \\
\hline pCDFDuet1 & lacla ${ }^{q}$, T7prom, spec ${ }^{R}$, CDFori & Novagen \\
\hline pSLBPSA3 & $\begin{array}{c}\text { pCDFDuet based staging vector allowing } \\
\text { introduction of foreign PCP domains into bpsA } \\
\text { with silent restriction sites. }\end{array}$ & (J. Owen 2012) \\
\hline pNOHISPET & $\begin{array}{l}\text { Modified form of pET28a(+) with the N-terminus } \\
6 \text { HIS tag removed to allow the expression of } \\
\text { PcpS without a 6HIS tag }\end{array}$ & This study \\
\hline pET::Sfp & $\mathrm{pET}-28 \mathrm{a}(+)+\mathrm{Sfp}$ & (J. Owen 2011) \\
\hline pET::HSfp & pET-28a $(+)+$ Human PPTase & This study \\
\hline pET::AcpS & pET-28a $(+)+$ M. tuberculosis AcpS & This study \\
\hline pET::ECAcpS & pET-28a $(+)+E$. coli AcpS & This study \\
\hline pET::PptT & pET-28a(+) + PptT & This study \\
\hline $\mathrm{pET}:: B p s A$ & pET-28a(+) + BpsA & (J. Owen 2011) \\
\hline pET::MPptT & pET-28a (+) + M. smegmatis PptT & This study \\
\hline pET::AcpH & pET-28a(+) + AcpH & This study \\
\hline pET::4OT & pET-28a(+) 4'OT & This study \\
\hline pET::PcpS & pET-28a(+) + PcpS & (J. Owen 2011) \\
\hline pET::EntF & pET-28a(+) + EntF A domain & (J. Owen 2010) \\
\hline pET::DLBpsA & pET28a $(+)+$ BpsA with the D/L swap mutation & This study \\
\hline NHP::PcpS & NOHISPET + PcpS & This study \\
\hline pCDF:BpsA & pCDFDuet1 + BpsA & (J. Owen 2011) \\
\hline
\end{tabular}

Table 2.2 Plasmids used in this study. 


\subsection{Oligonucleotide primers and synthesised genes}

Primers were designed using Geneious ${ }^{\circledR}$ or the Primer 3 Plus ${ }^{\circledR}$ web tool and ordered from IDT (Carlsbad, CA, USA) custom oligonucleotide service. Primers were reconstituted to a final concentration of $100 \mu \mathrm{M}$ in $1 \times \mathrm{TE}$ buffer for storage at $-20^{\circ} \mathrm{C}$. Working stocks were prepared by dilution to $10 \mu \mathrm{M}$ with filter sterilised $\mathrm{ddH}_{2} \mathrm{O}$. The names and sequences of all primers used in this study are given in Table 2.3.

\begin{tabular}{|c|c|}
\hline Primer Name & Sequence $\left(5^{\prime} \rightarrow 3^{\prime}\right)$ \\
\hline \multicolumn{2}{|l|}{ PPTase specific } \\
\hline \multicolumn{2}{|l|}{ primers } \\
\hline HS_Ppt_Fwd & CCCCCATATGGTTTTCCC \\
\hline HS_Ppt_Rev & CCCCCTCGAGTTAAGATTTG \\
\hline Mt_AcpS_Fwd & CCCCCATATGGGCATCGTCGGTGTG \\
\hline Mt_AcpS_Rev & CCCCCTCGAGTCACGGGGCCTCCAGGAT \\
\hline EC_AcpS_Fwd & CCCCCATATGGGCATCGTCGGTGTG \\
\hline EC_AcpS_Rev & CCCCCTCGAGTCACGGGGCCTCCAGGAT \\
\hline MT_PptT_Fwd & CCCCCATATGGACGGTAGGCACGCTG \\
\hline MT_PptT_Rev & CCCCCTCGAGTCATAGCACGATCGCGGT \\
\hline NHIS_PcpS_fwd & CCCCAAAGCTTATGCGCGCCATGAAC \\
\hline NHIS_PcpS_Rev & CCCCCTCGAGTCAGGCGCCGACCGC \\
\hline MS_PptT_Fwd & CCCCAGATCTATGACGGACAGCCTGCTT \\
\hline MS_PptT_Rev & CCCCAGATCTTCACAGGACGATCGCCGT \\
\hline \multicolumn{2}{|c|}{ Construction of BpsA } \\
\hline \multicolumn{2}{|l|}{ variants } \\
\hline SI_BpsA_Fwd & AGCTCTGCAGAGCTCGTCGAGCGCCCCTTCGTCGCCCCGCGCACG \\
\hline SI_BpsA_Rev & AGCTTCTAGACTCCTGGGCGACCTCGCGCTCCAGGCGGCGGGCCA \\
\hline \multicolumn{2}{|l|}{ Other proteins } \\
\hline IgiF_Fwd & CCCCCATATGGTGCCCCACATCCACGTC \\
\hline IgiF_Rev & CCCCGTCGAGTCAACTCTCGTTCCAGTAGCC \\
\hline \multicolumn{2}{|l|}{ D/L swap primers } \\
\hline FP_fwd & CTATCATATGACTCTTCAGGAGACCAGCGTGCTCGA \\
\hline FP_rev & CTATGGATCCTCACTCGCCGAGCAGGTAGCGGATGT \\
\hline IP_fwd & CCGATGAGCTTCGCCGACGCCCAGTGG \\
\hline IP_rev & CCACTGGGCGTCGGCGAAGCTCATCGG \\
\hline \multicolumn{2}{|c|}{ Sequencing primers } \\
\hline T7_Fwd & TAATACGACTCACTATAGGG \\
\hline T7_Rev & GCTAGTTATTGCTCAGCGG \\
\hline BpsA_T_Screen & ACGAGCAGATCGGCCACGA \\
\hline
\end{tabular}


For gene synthesis, Hs-Ppt was codon optimised by the IDT codon optimiser tool and synthesised by GenScript (Piscataway, NJ, USA). The modified PCP domains, acpBpsA and facpBpsA and the coding region of the $D / L$ BpsA swap were synthesised as gBlocks $^{\circledR}$ by Integrated DNA Technologies (IDT, Carlsbad, CA, USA). IgiF was codon optimised using the IDT codon optimiser webtool and synthesised by IDT (Carlsbad, CA, USA).

\subsection{Bacterial growth and maintenance}

\subsubsection{Media}

Unless otherwise noted, all media components were dissolved in $\mathrm{dd}_{2} \mathrm{O}$ and sterilised by autoclaving at $121^{\circ} \mathrm{C}$ for 15 minutes or filter sterilised using a $0.22 \mu \mathrm{m}$ syringe filter unit. Media was stored at room temperature or $4{ }^{\circ} \mathrm{C}$ if it contained heat sensitive supplements.

\subsubsection{Lysogeny Broth (LB)}

\begin{tabular}{ll}
\hline Component & Final \\
& Concentration \\
\hline Tryptone & $10 \mathrm{~g} \cdot \mathrm{L}^{-1}$ \\
Yeast extract & $\underline{5} \mathrm{~g} \cdot \mathrm{L}^{-1}$ \\
$\mathrm{NaCl}$ & $5 \mathrm{~g} \cdot \mathrm{L}^{-1}$ \\
\hline
\end{tabular}

*LB was supplied as a premixed powder by the manufacturer.

\subsubsection{Succinate Minimal Medium}

\begin{tabular}{ll}
\hline Component & $\begin{array}{l}\text { Final } \\
\text { Concentration }\end{array}$ \\
\hline $\mathrm{K}_{2} \mathrm{HPO}_{4}$ & $6.0 \mathrm{~g} . \mathrm{L}^{-1}$ \\
$\mathrm{KH}_{2} \mathrm{PO}_{4}$ & $3.0 \mathrm{~g} . \mathrm{L}^{-1}$ \\
$\left(\mathrm{NH}_{4}\right)_{2} \mathrm{SO}_{4}$ & $1.0 \mathrm{~g} . \mathrm{L}^{-1}$ \\
$\mathrm{MgSO}_{4}$ & $0.2 \mathrm{~g} . \mathrm{L}^{-1}$ \\
Sodium succinate & $4.0 \mathrm{~g} . \mathrm{L}^{-1}$ \\
\hline
\end{tabular}

${ }^{*} \mathrm{pH}$ was adjusted to 7.0 by the addition of $\mathrm{NaOH}$.

\subsubsection{Pigment producing medium}

\begin{tabular}{ll}
\hline Component & $\begin{array}{l}\text { Final } \\
\text { Concentration }\end{array}$ \\
\hline LB & $20 \mathrm{~g} \cdot \mathrm{L}^{-1}$ \\
L-glutamine & $14.6 \mathrm{~g} \cdot \mathrm{L}^{-1}$
\end{tabular}

*Pigment producing medium is rapidly cooled after autoclaving to help avoid the breakdown of L-glutamine. For small volumes L-glutamine power is mixed with autoclaved LB and filter sterilised. 


\subsubsection{Media supplements}

Antibiotic stock solutions were made up to $1000 x$ final concentration in the appropriate solvent and filter sterilised using a $0.22 \mu \mathrm{M}$ filter before being stored at $-20^{\circ} \mathrm{C}$.

Antibiotics were generally made in $\mathrm{dd}_{2} \mathrm{O}$ to a working stock concentration of either 50 $\mathrm{mg} \cdot \mathrm{ml}^{-1}$ or $100 \mathrm{mg} \cdot \mathrm{ml}^{-1}$. Kanamycin at a final concentration of $50 \mu \mathrm{g} \cdot \mathrm{ml}^{-1}$ was used for maintenance of pET-28a(+) based plasmids and spectinomycin was used for maintenance of pCDFDuet1 based plasmids at a final concentration of $100 \mu \mathrm{g} \cdot \mathrm{ml}^{-1}$. IPTG stocks were prepared to a final concentration of $100 \mathrm{mg}^{\mathrm{ml}} \mathrm{m}^{-1}$.

\subsubsection{Bacterial growth and storage}

Unless otherwise stated, bacteria were grown at $37^{\circ} \mathrm{C}$, and aerated at $200 \mathrm{rev} / \mathrm{min}$ when grown in liquid culture. For short term plasmid maintenance, bacteria on agar plates were stored at $4{ }^{\circ} \mathrm{C}$. For long term storage $500 \mu \mathrm{l}$ of overnight liquid culture was mixed in a $1: 1$ ratio with autoclaved $80 \%$ glycerol $(\mathrm{v} / \mathrm{v})$ and stored at $-80{ }^{\circ} \mathrm{C}$ indefinitely.

\subsection{Routine molecular biology}

\subsubsection{Polymerase chain reaction (PCR)}

PCR amplification reactions for cloning were performed using Phusion ${ }^{\mathrm{TM}}$ high fidelity polymerase. For other applications including colony screening, Biomix $\operatorname{Red}^{\mathrm{TM}}$ was employed. PCR reactions were set up according to the manufacturer's protocols. Parameters and additives were optimised as required for individual genes. For colony screening a small amount of each colony was picked from an agar plate to be used as a template.

\subsubsection{Overlap PCR}

For overlap PCR a method based on the protocols described in (Williams et al., 2014). Briefly, the two fragments to be combined were amplified by PCR as per Section 2.4.1 and then purified using the methods described in Section 2.4.3. The fragments were then combined in an equimolar ratio and a primerless PCR reaction was run for 10 cycles. One $\mu \mathrm{L}$ of the forward and $1 \mu \mathrm{L}$ of the reverse primer at a concentration of $10 \mu \mathrm{M}$ were added to the reaction, which was run for a further 25 cycles to allow the amplification of the full-length fragment. 


\subsubsection{Agarose gel electrophoresis}

To assess the size and quality of DNA products including those of PCR reactions, DNA was electrophoresed on a $1 \%(\mathrm{w} / \mathrm{v})$ agarose gel, containing $1 \mu \mathrm{g} \cdot \mathrm{ml}^{-1}$ ethidium bromide. Gels were electrophoresed submerged in 1X TAE buffer (40 mM Tris-acetate pH 8.0, 1 mM EDTA) at 100-140 V for 30-70 min depending on the purpose of the gel and product size. Hyperladder I (Bioline, London, UK) was run alongside the samples for size comparison and bands were visualised under ultra-violet (UV) light.

\subsubsection{PCR product purification}

PCR products were purified using a Zymo-Spin ${ }^{\mathrm{TM}}$ column (Zymo Research; Irvine, CA, USA) according to the manufacturer's instructions. Products were eluted in filter sterilised $\mathrm{ddH}_{2} \mathrm{O}$ and stored at $-20^{\circ} \mathrm{C}$.

For PCR purification using a gel purification method the product was electrophoresed on a $1 \%(w / v)$ agarose gel with SYBR ${ }^{\circledR}$ Safe (Thermo Fisher Scientific; Waltham, MA, USA) and then visualised on a blue light imager. The gel band was excised and purified using a Qiagen QIAquick Gel Extraction Kit (Venlo, Netherlands) according to the manufacturer's instructions.

\subsubsection{Restriction enzyme digests}

Restriction digests were performed according to the manufacturer's instructions and heat inactivated at $65^{\circ} \mathrm{C}$ for $20 \mathrm{~min}$ prior to use in further ligation reactions. Where enzymes were unable to be heat inactivated, digests were immediately cleaned and concentrated using a Zymo-Spin ${ }^{\mathrm{TM}}$ column and eluted in $10-20 \mu \mathrm{L}$ of $\mathrm{ddH}_{2} \mathrm{O}$. Digests that were not used immediately were stored at $-20^{\circ} \mathrm{C}$.

\subsubsection{Ligation reaction}

Ligations were performed according to the manufacturer's instructions, using a 1:3 or 1:6 molar ratio of vector to insert, with each reaction containing no more than $20 \mathrm{ng} / \mu \mathrm{L}$ of DNA. Reactions were incubated at $25^{\circ} \mathrm{C}$ for $90 \mathrm{~min}$ for routine cloning or overnight at $16{ }^{\circ} \mathrm{C}$ for longer inserts or ligations with large amounts of DNA. Ligations were then used to transform competent cells or else were stored at $-20^{\circ} \mathrm{C}$. 


\subsubsection{Bacterial cell transformation techniques}

Ligations were transformed into electrocompetent cells, movement of plasmids between strains used chemically competent cells.

\subsubsection{Preparation of chemically competent cells}

For routine plasmid transformation, the $E$. coli strain to be made competent was inoculated from a glycerol stock and grown in $3 \mathrm{ml} \mathrm{LB}$ amended with $10 \mathrm{mM} \mathrm{MgCl}_{2}$ and any relevant antibiotics at $37^{\circ} \mathrm{C}, 200 \mathrm{rev} / \mathrm{min}$ overnight. The following day $50 \mathrm{ml}$ of LB supplemented with $10 \mathrm{mM} \mathrm{MgCl}_{2}$ and any relevant antibiotics was inoculated from this overnight culture to a starting $\mathrm{OD}_{600}$ of 0.1 . Cells were incubated at $37^{\circ} \mathrm{C}, 200 \mathrm{rev} / \mathrm{min}$ until an $\mathrm{OD}_{600}$ of 0.4 - 0.6 was reached. Cells were then cooled on ice for $15 \mathrm{~min}$ and then centrifuged at $2700 \mathrm{~g}$ for $15 \mathrm{~min}$ at $4{ }^{\circ} \mathrm{C}$. The cell pellet was then re-suspended in $50 \mathrm{ml}$ of ice-cold $100 \mathrm{mM} \mathrm{MgCl} 2$ and centrifuged at $2700 \mathrm{~g}$ for $15 \mathrm{~min}$ at $4{ }^{\circ} \mathrm{C}$. The cell pellet was then resuspended in $50 \mathrm{ml}$ of ice-cold $100 \mathrm{mM} \mathrm{CaCl}_{2}$ and centrifuged at 2700 $g$ for $15 \mathrm{~min}$ at $4{ }^{\circ} \mathrm{C}$. The cell pellet was then resuspended in $1 \mathrm{ml}$ of ice-cold $85 \mathrm{mM}$ $\mathrm{CaCl}_{2}$ supplemented with $15 \%$ glycerol $(\mathrm{v} / \mathrm{v})$ and then transformed using the protocol described in Section 2.4.6.2. If they were not used immediately they were snap frozen at $-80^{\circ} \mathrm{C}$

\subsubsection{Transformation of chemically competent cells}

Frozen cell aliquots were removed from $-80^{\circ} \mathrm{C}$ storage and thawed on ice prior to addition of plasmid DNA. For each transformation, DNA in a volume of less than $10 \mu \mathrm{l}$ was added to $100 \mu \mathrm{l}$ of cells. This mixture was left on ice for at least $20 \mathrm{~min}$ after which transformation was induced by heat shock at $42^{\circ} \mathrm{C}$ for $90 \mathrm{~s}$. Cells were then returned to ice for 1-2 min and then recovered in $900 \mu \mathrm{LB}$ at $37^{\circ} \mathrm{C}, 200 \mathrm{rev} / \mathrm{min}$ for $60-90 \mathrm{~min}$. Finally, cells were spread on an LB agar plate supplemented with appropriate antibiotics and grown overnight at $37^{\circ} \mathrm{C}$.

\subsubsection{Preparation of electrocompetent cells}

The $E$. coli strain to be made competent was inoculated from a glycerol stock and grown in $10 \mathrm{ml} \mathrm{LB}$ supplemented with any relevant antibiotics at $37^{\circ} \mathrm{C}, 200 \mathrm{rev} / \mathrm{min}$ overnight. This overnight culture was then used to inoculate $400 \mathrm{ml} \mathrm{LB}$ containing any relevant antibiotics with a starting $\mathrm{OD}_{600}$ of 0.1 . The culture was grown until an $\mathrm{OD}_{600}$ of $0.35-0.4$ 
was reached, at which point it was transferred to 8 sterile $50 \mathrm{ml}$ tubes and cooled on ice for $30 \mathrm{~min}$. Cells were then centrifuged for $15 \mathrm{~min}$ at $2700 \mathrm{~g}, 4^{\circ} \mathrm{C}$. For the first wash step, pellets were washed with ice cold sterile $\mathrm{ddH}_{2} \mathrm{O}$ by gentle pipetting. The pellets were resuspended in $200 \mathrm{ml}$ ice cold sterile $10 \%$ glycerol. Cells were pelleted again through centrifugation, and this time the pellet was resuspended in $100 \mathrm{ml}$ ice cold sterile $10 \%$ glycerol. Cells were then finally collected through centrifugation and the pellet was resuspended in $300-500 \mu \mathrm{l}$ of ice cold sterile $10 \%$ glycerol. The $\mathrm{OD}_{600}$ of a $1 / 100$ dilution was then measured $\left(\mathrm{OD}_{600}\right.$ of $1.0=2.5 \times 10^{8}$ cells $\left./ \mathrm{ml}\right)$ and the final concentration of cells was adjusted to $2-3 \times 10^{10} \mathrm{cells} / \mathrm{ml}$ by the addition of further ice cold sterile $10 \%$ glycerol. Sixty $\mu$ l aliquots were transferred to pre-chilled $1.5 \mathrm{ml}$ microcentrifuge tubes on ice. Cell aliquots were then snap frozen in a metal tube block which had been pre-chilled to $-80^{\circ} \mathrm{C}$. Aliquots of competent cells were stored at $-80^{\circ} \mathrm{C}$ until needed.

\subsubsection{Transformation of electrocompetent cells}

Frozen cell aliquots were removed from $-80^{\circ} \mathrm{C}$ storage and thawed on ice. Plasmid DNA was then added to the cells, typically $10-50 \mathrm{ng}$ in $20 \mu \mathrm{L}$ of sterile $\mathrm{ddH}_{2} \mathrm{O}$. The contents of the tube were then gently mixed and transferred to an ice cold sterile $2 \mathrm{~mm}$ gap electroporation cuvette. Cells were then electroporated at $2.5 \mathrm{kV}, 25 \mu \mathrm{F}, 100 \Omega$, following which $900 \mu \mathrm{L}$ of room temperature LB was immediately added to the cuvette and gently inverted to mix. Cells were incubated at $37^{\circ} \mathrm{C}, 200 \mathrm{rev} / \mathrm{min}$ for $1 \mathrm{~h}$ for recovery. Finally, cells were plated on LB agar plates containing the appropriate antibiotics. One hundred $\mu$ of undiluted, as well as $1 / 10$ and $1 / 100$ dilutions, of cells were plated initially to determine the optimal dilution for single colonies to be obtained on agar plates. The remainder of the transformation was mixed in a 1:1 ratio with sterile $80 \%$ glycerol and stored at $-80^{\circ} \mathrm{C}$ until needed.

\subsubsection{Isolation of plasmid DNA}

Isolation of plasmid DNA was achieved using the High-Speed Plasmid Mini Kit (Geneaid Biotech Ltd; New Taipei City, Taiwan) according to the manufacturer's instructions. 


\subsubsection{DNA quantification and sequencing}

Measurement of the concentration and purity of DNA was achieved using a Nanodrop ND-1000 spectrophotometer (Thermo Fisher Scientific; Waltham, MA, USA), according to the manufacturer's instructions. All DNA sequencing was carried out by Macrogen Inc. (Seoul, South Korea) using Sanger sequencing technology.

\subsection{Protein expression and purification}

All proteins to be expressed were cloned into 6 His-tag vectors and transformed into the expression strain BL21 $\Delta$ entD for purification via nickel affinity chromatography, with the exception of $p c p S$ in pNOHISPET::PcpS which was only expressed in cell lysate and used in an un-purified form.

\subsubsection{Expression in LB at low temperatures}

All PPTases and BpsA were routinely expressed at low temperatures to aid with protein purification. A single colony or glycerol stock of the expression strain was used to inoculate $10 \mathrm{ml}$ LB supplemented with appropriate antibiotics and grown overnight at 37 ${ }^{\circ} \mathrm{C}, 200 \mathrm{rev} / \mathrm{min}$. The following day this overnight culture was used to inoculate a full baffled 2.5 L flask with $400 \mathrm{ml}$ of LB containing appropriate antibiotics, giving a final $\mathrm{OD}_{600}$ of 0.1 . This was incubated at $37^{\circ} \mathrm{C}, 200 \mathrm{rev} / \mathrm{min}$ until an $\mathrm{OD}_{600}$ of $0.6-0.8$ was reached. Cultures were then transferred to an ice bath for $30 \mathrm{~min}$ before addition of 0.5 mM IPTG. Cultures were then grown for an additional 24 hours at $18^{\circ} \mathrm{C} 200 \mathrm{rev} / \mathrm{min}$ before cells were harvested through centrifugation at $2700 \mathrm{~g}, 20 \mathrm{~min}, 4^{\circ} \mathrm{C}$. The supernatant was discarded and cell pellets were stored at $-80^{\circ} \mathrm{C}$ until required.

\subsubsection{Expression in LB containing betaine and sorbitol at low temperatures}

A single colony or glycerol stock of expression strain was used to inoculate $10 \mathrm{ml}$ of LB supplemented with appropriate antibiotics as well as $2.5 \mathrm{mM}$ glycine betaine and $1 \mathrm{M} \mathrm{D}$ sorbitol and grown overnight at $37^{\circ} \mathrm{C}, 200 \mathrm{rev} / \mathrm{min}$. The following day $400 \mathrm{ml}$ of the same medium was inoculated by the overnight culture to give a final $\mathrm{OD}_{600}$ of 0.1 . This was incubated at $37^{\circ} \mathrm{C}, 200 \mathrm{rev} / \mathrm{min}$ until an $\mathrm{OD}_{600}$ of 0.6-0.8 was reached. Cultures were then transferred to an ice bath for 30 min before addition of $0.5 \mathrm{mM}$ IPTG.

Cultures were then grown for an additional 24 hours at $18{ }^{\circ} \mathrm{C} 200 \mathrm{rev} / \mathrm{min}$ before cells 
were harvested through centrifugation at $2700 \mathrm{~g}, 20 \mathrm{~min}, 4^{\circ} \mathrm{C}$. The supernatant was discarded and cell pellets were stored at $-80^{\circ} \mathrm{C}$ until needed.

\subsubsection{Cell lysis and soluble fraction separation}

For protein purification, cell pellets were thawed and resuspended in $35 \mathrm{ml}$ of $1 \mathrm{x}$ His•bind ${ }^{\mathrm{TM}}$ binding buffer, minus imidazole. For BpsA purification this binding buffer contained $12.5 \%$ glycerol $(\mathrm{v} / \mathrm{v})$, and for PPTase purification this binding buffer contained $25 \%$ glycerol (v/v). For PptT purification $10 \mathrm{mM} \mathrm{MgCl}_{2}$ and $50 \mu \mathrm{M} \mathrm{CoA}$ was added to the bind buffer. Cells were lysed by 2-3 passages through a French press chamber at 40,000 psi. The French press chamber was cooled on ice prior to use and the binding buffer was kept ice cold. Following lysis, the soluble and insoluble fractions were separated by centrifugation at $26,000 \mathrm{~g} 20 \mathrm{~min}, 4^{\circ} \mathrm{C}$.

\subsubsection{Conversion of apo-BpsA to holo-BpsA}

Cell pellets were re-suspended in a modified binding buffer ( $5 \mathrm{mM}$ imidazole, $0.5 \mathrm{M}$ $\mathrm{NaCl}, 12.5 \%$ glycerol (v/v) and $20 \mathrm{mM}$ Tris- $\mathrm{Cl} \mathrm{pH} \mathrm{7.8).} \mathrm{The} \mathrm{resuspended} \mathrm{cell} \mathrm{pellets}$ containing expressed PcpS and apo-BpsA were mixed together and mechanically lysed using an ice-cold French Press Cell at 40,000 psi. The soluble fraction was collected by centrifugation $\left(26,000 \mathrm{~g}, 20 \mathrm{~min}, 4^{\circ} \mathrm{C}\right.$ ) and supplemented with $100 \mu \mathrm{L}$ of $50 \mathrm{mM}$ Coenzyme $\mathrm{A}(\mathrm{CoA})$ and incubated for $2 \mathrm{~h}\left(25^{\circ} \mathrm{C}, 200 \mathrm{rev} / \mathrm{min}\right)$ to facilitate the PcpS mediated attachment of 4'-phosphopantetheine arm derived from Coenzyme A to BpsA. The BpsA, PcpS and CoA mixture was then centrifuged $\left(26,000 \mathrm{~g}, 20 \mathrm{~min}, 4^{\circ} \mathrm{C}\right)$ to remove precipitated PcpS, prior to purification of the holo-BpsA by Ni-NTA chromatography as described in Section 2.5.5.1.

\subsubsection{Protein purification via Ni-NTA affinity chromatography}

\subsubsection{Purification of BpsA}

Purification of 6His-tagged proteins was achieved using Novagen's His•bind TM Ni-NTA chromatography kit. The following changes to the manufacturer's instructions were made for the purification of BpsA (due to the weak binding of BpsA to the resin). All elution buffers contained $12.5 \%$ glycerol $(\mathrm{v} / \mathrm{v})$ and all reagents were kept at ice cold temperatures for the duration of the purification. A large volume, approximately 6-7 $\mathrm{ml}$, of resin was used. The soluble fraction was collected after it passed through the resin, 
and passed through the column a second time. Columns were then washed using $50 \mathrm{ml}$ of standard binding buffer. The wash buffer step that is usually employed at this stage of purification (according to the manufacturer's instructions) was omitted for BpsA. For the elution, the resin was resuspended in $8 \mathrm{ml}$ elution buffer, and protein was eluted in an 8-10 $\mathrm{ml}$ total volume. Buffer exchange and concentration of the eluted protein was performed in $100 \mathrm{kDa}$ molecular mass cut-off Millipore Amicon ${ }^{\circledR}$ ultra-15 centrifugal filter units (Merck Millipore, Billerica, MA, USA), into $50 \mathrm{mM}$ sodium phosphate buffer, $\mathrm{pH} 7.8,12.5 \%(\mathrm{v} / \mathrm{v})$ glycerol. Final buffer composition was then adjusted to $40 \%(\mathrm{v} / \mathrm{v})$ glycerol and aliquots were stored at $-20^{\circ} \mathrm{C}$.

\subsubsection{Purification of PPTases}

Amendments were also made to the standard protocol for PPTase purification, due to the instability and low yield typically observed with these proteins. All elution buffers contained $25 \%$ glycerol (v/v) and all reagents and equipment were kept at $4{ }^{\circ} \mathrm{C}$ for the duration of the experiment. The soluble fraction for PPTase purification was passed through the resin column. Columns were washed using 10-15 $\mathrm{ml}$ binding buffer (no imidazole), 10-15 $\mathrm{ml}$ standard binding buffer, 10-15 $\mathrm{ml}$ wash buffer (half standard imidazole concentration). Following elution, protein was immediately desalted using GE Healthcare HiTrap ${ }^{\mathrm{TM}}$ desalting columns, according to the manufacturer's protocol. Desalting buffer for PPTases was 50 mM Tris-Cl pH 7.8, 12.5\% glycerol (v/v). Glycerol was added to desalted protein to bring final concentration to $50 \%(\mathrm{v} / \mathrm{v})$ glycerol and aliquots were stored at $-80^{\circ} \mathrm{C}$.

\subsubsection{Purification of other proteins}

Other proteins such as CP domains and IgiF were purified in the same manner as for the PPTases, with adjustments made to the final storage buffer. CP domains were stored in $50 \mathrm{mM}$ Tris-Cl pH 8.0 supplemented with $40 \%$ (v/v) glycerol. IgiF was stored in $50 \mathrm{mM}$ sodium phosphate buffer pH 7.2 supplemented with $40 \%$ (v/v) glycerol.

\subsubsection{Protein concentration quantification}

The final concentration of a purified protein was quantified either using a $\mathrm{DC}^{\mathrm{TM}}$ protein assay kit (Bio-Rad, Hercules, CA, USA), following the manufacturer's instructions. Protein standards were made with bovine serine albumin (BSA) in the appropriate buffer and 
glycerol concentrations, ranging from $0.25-1.5 \mathrm{mg} \mathrm{ml}^{-1}$. Alternatively, protein concentrations were determined using absorbance at $280 \mathrm{~nm}$ using a Nanodrop ND1000 spectrophotometer (Thermo Fisher Scientific), according to the manufacturer's instructions.

\subsection{SDS-PAGE analysis}

For qualitative assessment of proteins, $12.5 \%$ or $15 \%$ SDS PAGE gels were run to visualise proteins depending on the protein size. These were cast and electrophoresed on a $1 \mathrm{mM}$ thickness Bio-Rad Protean $\mathrm{II}^{\mathrm{TM}}$ apparatus. For each gel, $5 \mathrm{ml}$ of separating gel was loaded into the apparatus before being overlaid with isopropanol and left to set for $45 \mathrm{~min}$. The isopropanol was then removed and $1.5 \mathrm{ml}$ of stacking gel was poured on top. A multi- well comb was inserted and this was left to set for at least $45 \mathrm{~min}$. If not used immediately, gels were stored for up to three days at $4{ }^{\circ} \mathrm{C}$, wrapped in a paper towel soaked in $\mathrm{dd}_{2} \mathrm{O}$ and tin foil. Samples were added to $3 x$ loading buffer in a 2:1 ratio, and incubated at $95{ }^{\circ} \mathrm{C}$ for $10 \mathrm{~min}$. Gels were electrophoresed in 1x SDS run buffer, at a constant voltage (180 V) for $60-75 \mathrm{~min}$, until the pre-stained ladder had reached proper separation. Gels were then stained in Coomassie blue stain and gentle shaking was applied for 30-60 min. Gels were then rinsed using tap water and left in destain solution with gentle rocking until the desired level of destain was reached. All recipes for SDS protocol can be found in table 2.4 .

\begin{tabular}{ll}
\hline Component & Final concentration \\
\hline 15\% separating gel & Per $10 \mathrm{ml}$ \\
40\% acrylamide solution & $3.65 \mathrm{ml}$ \\
2\% Bis-acrylamide solution & $2.0 \mathrm{ml}$ \\
$1.5 \mathrm{M} \mathrm{Tris-Cl} \mathrm{pH} 8.8$ & $2.5 \mathrm{ml}$ \\
$10 \%$ SDS & $100 \mu \mathrm{l}$ \\
$10 \%$ APS* & $100 \mu \mathrm{l}$ \\
TEMED* & $6 \mu \mathrm{l}$ \\
dd $\mathrm{H}_{2} \mathrm{O}$ & $1.75 \mathrm{ml}$
\end{tabular}




\section{$12.5 \%$ Separating gel}

$40 \%$ acrylamide solution

$2 \%$ Bis-acrylamide solution

1.5 M Tris-Cl pH 8.8

$10 \%$ SDS

$10 \%$ APS*

TEMED*

$\mathrm{dd}_{2} \mathrm{O}$

\section{5\% stacking gel}

$40 \%$ acrylamide solution

$2 \%$ Bis-acrylamide solution

0.5 M Tris-Cl pH 6.8

$10 \%$ SDS

$10 \%$ APS*

TEMED*

$\mathrm{dd}_{2} \mathrm{O}$

\section{3x SDS loading buffer}

Tris-Cl pH 6.8

SDS

Bromophenol blue

Glycerol

$\beta$-mercaptoethanol

$1 x$ run buffer

Glycine

Tris

SDS
Per $10 \mathrm{ml}$

$3.04 \mathrm{ml}$

$1.62 \mathrm{ml}$

$2.28 \mathrm{ml}$

$100 \mu \mathrm{l}$

$76 \mu \mathrm{l}$

$7.5 \mu \mathrm{l}$

$2.86 \mathrm{ml}$

\section{Per $6 \mathrm{ml}$}

$310 \mu \mathrm{l}$

$580 \mu \mathrm{l}$

$1.5 \mathrm{ml}$

$60 \mu \mathrm{l}$

$46 \mu \mathrm{l}$

$7.5 \mu \mathrm{l}$

$3.5 \mathrm{ml}$

\section{Final concentrations}

$150 \mathrm{mM}$

$6 \%(w / v)$

$0.3 \%(\mathrm{w} / \mathrm{v})$

$30 \%(w / v)$

$300 \mathrm{mM}$

Per $1 \mathrm{~L}$

$14.4 \mathrm{~g}$

$3.03 \mathrm{~g}$

$1 \mathrm{~g}$ 


\begin{tabular}{ll} 
Coomassie blue stain & Per 1 L \\
Coomassie brilliant blue & $2.5 \mathrm{~g}$ \\
100\% ethanol & $450 \mathrm{ml}$ \\
$100 \%$ acetic acid & $100 \mathrm{ml}$ \\
$\mathrm{ddH}_{2} \mathrm{O}$ & $450 \mathrm{ml}$ \\
& \\
\hline Destain solution & Per $1 \mathrm{~L}$ \\
100\% methanol & $400 \mathrm{ml}$ \\
Acetic acid & $100 \mathrm{ml}$ \\
dd $_{2} \mathrm{O}$ & $500 \mathrm{ml}$
\end{tabular}

Table 2.4 SDS-Page recipe: *Added immediately prior to pouring gel, APS is Ammonium persulfate, TEMED is Tetramethylethylenediamine

\subsection{In vitro assays for the detection of PPTase inhibition}

For ease of reading, methods have been grouped together based on chapter, rather than assay type.

\subsubsection{PPTase mediated conversion of apo-BpsA to holo-BpsA}

A master mix containing $50 \mathrm{mM}$ Tris-Cl pH 7.8, 10 mM MgCl , 4 mM ATP, 4 mM L-gln, 1 $\mu \mathrm{M}$ BpsA and $5 \mu \mathrm{M}$ CoA and $\mathrm{ddH}_{2} \mathrm{O}$ in a final volume of $80 \mu \mathrm{L}$ was added in triplicate to a standard 96 well plate in triplicate. To initiate the reaction a PPTase at a range of concentrations (dependant on the experiment and type of PPTase) in final volume of 20 $\mu \mathrm{L}$ in $\mathrm{dd}_{2} \mathrm{O}$ was rapidly dispensed into each well. The 96 well plate was then shaken at $1000 \mathrm{rev} / \mathrm{min}$ and the $A_{590}$ values were recorded every $10-20 \mathrm{~s}$ at $590 \mathrm{~nm}$ using a PerkinElmer EnSpire ${ }^{\circledR}$ plate reader depending on the speed of the reaction. The $A_{590}$ values were then graphed against time using GraphPad Prism ${ }^{\circledR}$.

\subsubsection{Optimised inhibitor screening protocol}

Twenty eight $\mu \mathrm{L}$ of $10 \%$ DMSO was aliquoted into columns 1 - 11 of a standard 96 well plate. The plate was then mixed so that the bottom of the well was entirely covered. $A$ $\mathrm{CyBio}^{\circledR}$ liquid handling robot was then used to aliquot $2 \mu \mathrm{L}$ of drug from a 96 well drug plate into columns $2-10$ to give a final concentration of $20 \mu \mathrm{M}$. A master mix containing 
50 mM Tris-Cl pH 8.0, 10 mM MgCl 2,6 mM ATP, 6 mM L-gln, $2 \mu \mathrm{M}$ apo-BpsA, $5 \mu \mathrm{M}$ CoA in $\mathrm{dd}_{2} \mathrm{O}$ with a final volume of $50 \mu \mathrm{L}$ was dispensed into each well. To initiate the reaction $0.4 \mu \mathrm{M}$ of PptT or $0.2 \mu \mathrm{M}$ of PcpS in $\mathrm{ddH}_{2} \mathrm{O}$ in a final volume in $20 \mu \mathrm{L}$ was dispensed rapidly into each well other than the negative control. The 96 well plate was then shaken for $10 \mathrm{~s}$ at $1000 \mathrm{rev} / \mathrm{min}$ and immediately read in a PerkinElmer EnSpire ${ }^{\circledR}$ plate reader at $590 \mathrm{~nm}$ every $20 \mathrm{~s}$ for $25-30$ repeats. The A59o values were then graphed against time using Microsoft Excel ${ }^{\circledR}$ and ranked based on the level of inhibition.

\subsubsection{Counter screening against holo-BpsA}

Holo-BpsA was expressed and purified as described in methods Section 2.5. Screening took place in the same manner as described in 2.9.2 except apo-BpsA was replaced with holo-BpsA in the master mix. L-gln was removed from the master mix. To initiate the reaction $6 \mathrm{mM} \mathrm{L-gln} \mathrm{in} 20 \mu \mathrm{L}$ of $\mathrm{ddH}_{2} \mathrm{O}$ was dispensed to each well instead of a PPTase.

\subsubsection{Determination of inhibitor $\mathrm{IC}_{50}$ values}

A two-fold serial dilution of a candidate inhibitor from $200 \mu \mathrm{M}$ was set up in a 96 well plate in triplicate, in $30 \mu \mathrm{L}$ of $10 \%$ DMSO. The 96 well plate was then screened as described in Section 2.7.2. A590 values were recorded every $10-20 \mathrm{~s}$ depending on the reaction velocity. Data was analysed in Microsoft Excel ${ }^{\circledR}$ using the slope function to derive velocity values. The slope function was then applied to these velocity values, and the maximum value for each reaction taken as the maximum PPTase velocity. For reactions with lower velocities (e.g. more inhibition), the slope function was applied across a larger number of data points. The maximum velocity for each inhibitor concentration had a four parameter dose response curve fitted using GraphPad Prism ${ }^{\circledR}$ to derive an $\mathrm{IC}_{50}$ value. The procedure for kinetic analysis is described in full in Owen et al. (2011).

\section{8 in vivo quantification of PPTase inhibition}

A $5 \mathrm{ml} \mathrm{LB}$ culture was inoculated with $P$. aeruginosa PA01 from a glycerol stock and incubated at $37^{\circ} \mathrm{C} 200 \mathrm{rev} / \mathrm{min}$ for $16 \mathrm{~h}$. The culture was then pelleted at $2700 \mathrm{~g}$ for 15 $\min$. The cell pellet was resuspended in $5 \mathrm{ml}$ of minimal media and the $\mathrm{OD}_{600}$ value was spectroscopically determined. The $\mathrm{OD}_{600}$ value of the culture was then adjusted to 0.1 . A 
two-fold serial dilution in triplicate of the compound to be tested was established in 100 $\mu \mathrm{L}$ of minimal media across the 96 well plate. $100 \mu \mathrm{l}$ of the culture with the $\mathrm{OD}_{600}$ adjusted to 0.1 was then dispensed into to each well, giving a final OD600 value of 0.05 . The plate was incubated inside a PerkinElmer EnSpire ${ }^{\circledR}$ plate reader at $37^{\circ} \mathrm{C}, 200$ $\mathrm{rev} / \mathrm{min}$. The $\mathrm{OD}_{600}$ and fluorescent values (ex. $400 \mathrm{~nm} / \mathrm{em} .440 \mathrm{~nm}$ ) were read every 30 min. Growth curves were graphed against time using GraphPad Prism ${ }^{\circledR}$.

\subsection{Directed evolution of BpsA}

The following directed evolution protocols, which were used to try and improve activity between TB-AcpS and BpsA, were adapted from Owen (2010).

\subsubsection{Vector preparation}

The slBpsA plasmid was isolated using a Geneaid $^{\circledR}$ miniprep kit according to the manufacturer's instructions. Approximately $12 \mathrm{ml}$ of overnight culture was used to purify $20 \mu \mathrm{g}$ of vector. For digestion, $16 \mu \mathrm{g}$ plasmid DNA was heated to $70{ }^{\circ} \mathrm{C}$ for $20 \mathrm{~min}$, to relieve supercoiling and then digested using $50 \mathrm{U}$ of each restriction enzyme (Spel and $\mathrm{Nsil}$ ) in a final volume of $400 \mu \mathrm{L}$, following the manufacturer's instructions for buffer composition and temperature. After $6 \mathrm{~h}$ incubation a further $20 \mathrm{U}$ of each enzyme was added and reactions were incubated for an additional $16 \mathrm{~h}$. Digests were then heat inactivated at $80^{\circ} \mathrm{C}$ for $20 \mathrm{~min}$ and purified using a $20 \mu \mathrm{g}$ capacity Zymo-Spin ${ }^{\mathrm{TM}}$ clean up column. Digested vector was eluted in $30 \mu$ sterile $d_{d H_{2}} \mathrm{O}$ and the concentration of the digested vector was determined. A $200 \mathrm{ng}$ sample was run on an agarose gel to ensure no degradation had occurred. The rest of the digestion was then aliquoted in $5 \mu l$ portions and stored at $-20{ }^{\circ} \mathrm{C}$ until required.

\subsubsection{Assessment of vector quality}

It was important to assess vector quality before use in directed evolution library generation experiments as we would be selecting for colonies that produced a blue pigment rather than using a selection based screen, this meant it was important to have a high insert rate. The bpsA PCP domain was amplified using Phusion ${ }^{\text {TM }}$ high fidelity polymerase and prepared for ligation as described in Section 2.9.5. A ligation using a total of 500 ng DNA was set up as in Section 2.4.5, using the Phusion ${ }^{\mathrm{TM}}$ amplified PCP 
domain in place of the error prone PCR (epPCR) amplified PCP domain. This ligation was then used to transform electrocompetent competent BL21 $\Delta$ entD cells harbouring a pET-28a(+)::pcpS plasmid. The transformation was plated onto LB agar plates containing $115 \mathrm{mM} \mathrm{L-glutamine,} \mathrm{kanamycin} \mathrm{and} \mathrm{spectinomycin.} \mathrm{Plates} \mathrm{were} \mathrm{incubated} \mathrm{at} 37^{\circ} \mathrm{C}$ overnight and BpsA was induced as described in Section 2.9.6. Plates were incubated at room temperature. Transformation and ligation efficiency were determined by comparing numbers of blue colonies vs. white colonies after 8-12 h. Blue colonies indicated a successful ligation, while white indicated a self-ligation or uncut vector. A successful ligation rate of $70 \%$ or higher was deemed sufficient for use in further experiments.

\subsubsection{Error prone PCR}

epPCR was carried out on the bpsA PCP domain using the GeneMorph ${ }^{\circledR}$ Mutazyme $^{\circledR}$ II kit, according to the manufacturer's instructions. Template was prepared by amplification of the PCP domain using Phusion ${ }^{\mathrm{TM}}$ polymerase. A high error rate was chosen, which was achieved through use of $1 \mathrm{ng}$ purified PCR product as template in a $50 \mu \mathrm{l}$ reaction and 35 amplification cycles. Prior to thermocycling, reactions were divided into equal amounts $(12.5 \mu \mathrm{l})$ across 4 tubes to reduce likelihood of clonal mutations in the final library. The size and quality of the amplicons was determined by running a $3 \mu l$ aliquot of total pooled reactions on an agarose gel.

\subsubsection{Insert preparation}

The $50 \mu$ lepPCR reactions were purified using $5 \mu$ g capacity Zymo-Spin ${ }^{\text {TM }}$ columns, and eluted in $25 \mu$ sterile $d d \mathrm{H}_{2} \mathrm{O}$. The concentration of each purified reaction was determined by analysing a $1.2 \mu \mathrm{l}$ aliquot on a Nanodrop ND-1000 spectrophotometer. The rest of the eluent was then digested using $30 \mathrm{U}$ of each restriction enzyme (Pstl and Xbal), for $5 \mathrm{~h}$, in a final volume of $50 \mu \mathrm{l}$. Buffer composition and temperature were as recommended by the manufacturer. The digest was then heat inactivated by incubation at $80^{\circ} \mathrm{C}$ for $30 \mathrm{~min}$.

\subsubsection{Ligation and transformation}

The total amount of DNA per ligation was 1- $1.5 \mu \mathrm{g}$, with a molar ratio of vector to insert of $1: 6$. Ligations were incubated at $22^{\circ} \mathrm{C}$ for $1 \mathrm{~h}$ and then subsequently at $16^{\circ} \mathrm{C}$ 
overnight. Following this, an additional 2 units of ligase was added and the reaction was incubated for a further $7 \mathrm{~h}$. Ligations were purified using a $5 \mu \mathrm{g}$ capacity Zymo-Spin ${ }^{\mathrm{TM}}$ column and eluted in $25 \mu \mathrm{l}$ of $\mathrm{ddH}_{2} \mathrm{O}$. Ligations were used to transform electrocompetent BL21 $\Delta$ entD cells harbouring pET28a(+)::TB-acpS. Electrocompetent cells were prepared and transformed as described in Sections 2.4.6.3 and 2.4.6.4.

\subsubsection{First Tier screening}

A small aliquot of the library was defrosted on ice and a serial dilution was plated out on pigment producing media to empirically determine the optimal plating volume to achieve $4000-8000$ colonies per plate. The library was then plated at this density. After $12-14 \mathrm{~h}$ of incubation at $37^{\circ} \mathrm{C}$, expression was induced in plates. Induction was achieved by lifting the agar out of the plate and spreading $120 \mu \mathrm{L}$ of $3 \%$ IPTG on the bottom of the agar plate and then replacing the agar back into the plate which allowed the IPTG diffuse through the agar. Plates were then incubated at $20{ }^{\circ} \mathrm{C}$ and monitored for the production of pigment. Colonies that produced detectable levels of indigoidine were picked and restreaked. The re-streaking reduced the chance of contamination for colonies that were closely grouped together and provided a more quantitative estimate of the time taken to produce detectable levels of pigment as this was found to vary according to cell density and position on the plate. Subsequent induction of this plate allowed recovery of improved clones without contaminants. Improved clones were then grown overnight in LB medium supplemented with $0.4 \%$ glucose and appropriate antibiotics. Glycerol stocks of improved clones were then prepared in a 96 well plate.

\subsubsection{Second tier screening}

Glycerol stocks of appropriate strains were used to inoculate $200 \mu$ l of LB containing appropriate antibiotics and then incubated overnight at $37^{\circ} \mathrm{C}, 200 \mathrm{rev} / \mathrm{min}$ in a 96 well plate. Twenty $\mu$ l of each culture were used to inoculate a fresh 96-well microplate, with each well containing $130 \mu \mathrm{LB}$ amended with $100 \mathrm{mM}$ L-glutamine, $0.6 \mathrm{mM}$ IPTG, and appropriate antibiotics. Plates were wrapped in foil and incubated at $18^{\circ} \mathrm{C}, 200 \mathrm{rev} / \mathrm{min}$ for 24-48 h. At appropriate time points based on visual inspection, $\mathrm{OD}_{590}$ and $\mathrm{OD}_{800}$ values were recorded using an PerkinElmer Enspire plate reader. After a reading was taken the plate was again incubated at $18{ }^{\circ} \mathrm{C}, 200 \mathrm{rev} / \mathrm{min}$. 


\subsubsection{Quantification and normalisation of indigoidine levels}

A normalised value for indigoidine absorbance was calculated using the method described by Beer et al (2014), as adapted from Myers et al (2013). The OD values recorded represent the indigoidine sensitive wavelength $\left(O D^{S}\right)$ of $590 \mathrm{~nm}$; as well as the robust wavelength $\left(O D^{R}\right)$ of $800 \mathrm{~nm}$, which accounts for the cellular components present. $O D^{S_{B}+1}$ is the $O D$ at $590 \mathrm{~nm}$ which contains absorption from both bacterial cells and the indigoidine pigment, while $\mathrm{OD}_{\mathrm{B},-}^{\mathrm{S}}$ accounts for the scattering of just the cellular components at $O D^{S}$ (i.e. at $590 \mathrm{~nm}$ ). $O D^{S}{ }_{B,-1}$ is calculated as:

$$
O D^{S} S_{-1}=\delta . O D^{R}
$$

where $\delta$ is the correction factor and is calculated by measuring the ratio of pure cell culture at both $O D^{S}(590 \mathrm{~nm})$ and $O D^{R}(800 \mathrm{~nm})$. The normalised calculation for indigoidine is then calculated as:

$$
\text { relative indigoidine }=O D_{B,+1}^{S}-\delta . O D^{R}
$$

Background correction was achieved by subtracting the mean of "culture medium alone" replicates from every value first.

\subsection{In vitro L-glutamine and ATP assays}

The following methods are summarised from Brown et al (2017).

\subsubsection{Kinetic analysis of holo-BpsA}

To confirm that BpsA had been converted to the active holo form, the rate of indigoidine synthesis was measured. A reaction mix containing $\mathrm{ddH}_{2} \mathrm{O}, 50 \mathrm{mM}$ Tris- $\mathrm{Cl} \mathrm{pH} \mathrm{8.5,} 20 \mathrm{mM}$ $\mathrm{MgCl}_{2}, 6 \mathrm{mM}$ ATP and $1 \mu \mathrm{M} \mathrm{BpsA}$ in a total volume of $90 \mu \mathrm{L}$ was added to individual wells of a 96 well plate in triplicate. The reaction was initiated by the addition of $10 \mu \mathrm{L}$ of $1 \mathrm{mM} \mathrm{L}$-glutamine in $\mathrm{ddH}_{2} \mathrm{O}$, giving a final reaction volume of $100 \mu \mathrm{L}$ per well. The 96 well plate was mixed at $1000 \mathrm{rev} / \mathrm{min}$ for $15 \mathrm{~s}$ and $A_{590}$ values were recorded every $10 \mathrm{~s}$ for $1 \mathrm{~h}$. The reaction rate was calculated by measuring the maximum reaction velocity $\left(\Delta \mathrm{A}_{590 \mathrm{~S}^{-1}}\right)$. 


\subsubsection{Kinetic analysis of L-glutamine samples}

To estimate the concentration of glutamine samples without resolubilising the indigoidine produced, the maximum absorbance achieved across a range of concentrations was measured. A reaction mix containing: $\mathrm{dd}_{2} \mathrm{O}, 50 \mathrm{mM}$ Tris- $\mathrm{Cl}$ pH 8.5, $20 \mathrm{mM} \mathrm{MgCl}_{2}, 6 \mathrm{mM} \mathrm{ATP}$ and $2 \mu \mathrm{M}$ holo-BpsA in a total volume of $90 \mu \mathrm{L}$ was added to a 96 well plate in triplicate. The reaction was initiated by the rapid addition of $10 \mu \mathrm{L}$ of $\mathrm{L}-$ glutamine standards spanning a range of concentrations $(0-1000 \mu \mathrm{M})$. The 96 well plate was mixed at $1000 \mathrm{rev} / \mathrm{min}$ for $15 \mathrm{~s}$ and $A_{590}$ values were measured every $10 \mathrm{~s}$ for $1 \mathrm{~h}$. The maximum $A_{590}$ value achieved for each sample within this time was recorded and this was used to generate a standard curve.

\subsubsection{Optimisation of DMSO stop method}

The DMSO stop protocol comprises two parts. In the first part holo-BpsA converts two molecules of glutamine into indigoidine. A reaction mix containing the following was set up: $50 \mathrm{mM}$ Tris- $\mathrm{Cl} \mathrm{pH} \mathrm{8.5,} 20 \mathrm{mM} \mathrm{MgCl}_{2}, 6 \mathrm{mM}$ ATP and $3 \mu \mathrm{M}$ holo-BpsA in ddH $\mathrm{H}_{2} \mathrm{O} .30 \mu \mathrm{L}$ of reaction mix was added in triplicate to a 96 well plate containing $10 \mu \mathrm{L}$ of L-glutamine standards spanning a range of concentrations $(0-1000 \mu \mathrm{M})$ in $\mathrm{dd}_{2} \mathrm{O}$ to initiate the reaction. The 96 well plate was then incubated $\left(25^{\circ} \mathrm{C} 200 \mathrm{rev} / \mathrm{min}\right.$ for $60 \mathrm{~min}$ ). Once all the L-glutamine had been converted to indigoidine it was re-solubilised by the addition of $200 \mu \mathrm{L}$ anhydrous DMSO (final concentration 83\% (v/v)). The 96 well plate was then shaken at $2000 \mathrm{rev} / \mathrm{min}$ for $20 \mathrm{~min}$ and the $A_{590}$ value for each well was recorded. Triplicate reactions were averaged and used to generate a standard curve. For each of the different optimisation steps individual parameters were varied including; incubation time, DMSO concentration and BpsA concentration to ensure complete conversion of Lglutamine to indigoidine and complete re-solubilisation of indigoidine.

\subsubsection{Measurement of glutamine in a range of relevant conditions}

For measurement of L-glutamine in LB, Dulbecco's Modified Eagle's Medium (DMEM) or Molecular, Cellular, and Developmental Biology (MCDB) medium, no additional sample processing was needed. For human blood (collected using a purple vacutainer blood collection tube which contains potassium salt of EDTA as an anticoagulant) and human 
urine the samples were first centrifuged $\left(13,000 \mathrm{~g}, 2 \mathrm{~min}, 25^{\circ} \mathrm{C}\right)$ to remove cells. The blood sample also required additional processing. The plasma was passed through a 3000 Dalton cut off column via centrifugation $\left(2,700 \mathrm{~g}, 30 \mathrm{~min}, 25^{\circ} \mathrm{C}\right)$ to deproteinate the sample. L-glutamine standards $(0-1000 \mu \mathrm{M})$ were prepared in $\mathrm{ddH}_{2} \mathrm{O} .30 \mu \mathrm{L}$ of reaction mix (as described in Section 2.10.3) was added to $10 \mu \mathrm{L}$ of sample to initiate the reaction. The 96 well plate was then incubated $\left(25^{\circ} \mathrm{C}, 200 \mathrm{rev} / \mathrm{min}, 60 \mathrm{~min}\right)$. The indigoidine was then re-solubilised by the addition of $200 \mu \mathrm{L}$ of DMSO. The 96 well plate was mixed ( $2000 \mathrm{rev} / \mathrm{min}, 20 \mathrm{~min}$ ) and the $A_{590}$ value was recorded. Each reaction was repeated in triplicate and the $A_{590}$ values averaged to generate the final value. $A$ standard curve was generated from the standards and this was used to extrapolate the calculated glutamine concentrations for the different samples.

\subsubsection{Long term stability}

The long-term stability tests at different temperatures were performed on apo-BpsA which was converted to holo-BpsA immediately prior to testing. Three samples were stored for 24 weeks in storage buffer $(40 \%(\mathrm{v} / \mathrm{v})$ glycerol, $50 \mathrm{mM}$ sodium phosphate buffer $\mathrm{pH} 7.8$ ) at $-20^{\circ} \mathrm{C}, 4^{\circ} \mathrm{C}$ or $25^{\circ} \mathrm{C}$. A reaction mix containing the following reagents was generated to convert the apo-BpsA to holo-BpsA: $2 \mu \mathrm{M}$ apo-BpsA, $12.5 \mu \mathrm{M}$ Coenzyme A, $0.1 \mu \mathrm{M}$ Sfp, $5 \mathrm{mM} \mathrm{MgCl}$, $50 \mathrm{mM}$ Tris- $\mathrm{Cl} \mathrm{pH} 7.8$ and $\mathrm{dd}_{2} \mathrm{O}$. A total volume of $25 \mu \mathrm{L}$ per reaction was incubated at $30^{\circ} \mathrm{C} 200 \mathrm{rev} / \mathrm{min}$ for $30 \mathrm{~min} .25 \mu \mathrm{L}$ of this reaction mix was dispensed into a 96 well plate containing $50 \mu \mathrm{L}$ of $50 \mathrm{mM}$ Tris- $\mathrm{Cl} \mathrm{pH} 7.8$ and $5 \mathrm{mM} \mathrm{MgCl} 2$ in $\mathrm{ddH}_{2} \mathrm{O}$. Indigoidine synthesis was initiated by the addition of $25 \mu \mathrm{L}$ of $5 \mathrm{mM}$ ATP and $2 \mathrm{mM}$ L-glutamine in $\mathrm{dd}_{2} \mathrm{O}$ (concentrations are per final $100 \mu \mathrm{L}$ reaction volume). The 96 well plate was then shaken at $1000 \mathrm{rev} / \mathrm{min}$ for $10 \mathrm{~s}$ and the $A_{590}$ values were recorded every $10 \mathrm{~s}$ for $1 \mathrm{~h}$. The initial velocity of the reaction was calculated by finding the maximum slope value (as previously described in (Owen et al., 2011).

The 11 month stability test was performed using the same preparation of holo-BpsA. A master mix containing the following was dispensed into a 96 well plate $2 \mu \mathrm{M}$ holo-BpsA, $50 \mathrm{mM}$ Tris- $\mathrm{Cl} \mathrm{pH} \mathrm{8.5,} 20 \mathrm{mM} \mathrm{MgCl}_{2}$ and $5 \mathrm{mM}$ ATP and $\mathrm{ddH}_{2} \mathrm{O}$ with a final volume of 90 $\mu \mathrm{L}$. The reaction was initiated by the addition of $10 \mu \mathrm{L}$ of $1000 \mu \mathrm{M} \mathrm{L}$-glutamine in $\mathrm{dd}_{2} \mathrm{O}$. The 96 well plate was then shaken at $1000 \mathrm{rev} / \mathrm{min}$ for $10 \mathrm{~s}$ and the $A_{590}$ values were 
recorded every $10 \mathrm{~s}$ for $1 \mathrm{~h}$. The velocity of the reaction was calculated by finding the maximum slope value as above. The holo-BpsA was stored for 11 months at $-20^{\circ} \mathrm{C}$ in storage buffer. The sample was then tested in the same manner and the maximum velocity was calculated.

\subsubsection{Assay for the quantification of ATP}

For measurement of ATP in $\mathrm{dd}_{2} \mathrm{O}$, LB or RPMI. ATP standards $(0-1000 \mu \mathrm{M})$ were prepared in $\mathrm{ddH}_{2} \mathrm{O}$, LB or Roswell Park Memorial Institute (RPMI) media depending on the sample being tested. $30 \mu \mathrm{L}$ of reaction mix $\left(50 \mathrm{mM}\right.$ Tris- $\mathrm{Cl}$ pH 8.5, $20 \mathrm{mM} \mathrm{MgCl}_{2}, 6$ mM L-glutamine and $3 \mu \mathrm{M}$ holo-BpsA in $\mathrm{dd}_{2} \mathrm{O}$ ) was added to $10 \mu \mathrm{L}$ of sample to initiate the reaction. The 96 well plate was then incubated $\left(25^{\circ} \mathrm{C}, 200 \mathrm{rev} / \mathrm{min}, 60 \mathrm{~min}\right)$. The indigoidine was then re-solubilised by the addition of $200 \mu \mathrm{L}$ of DMSO. The 96 well plate was mixed (2000 rev/min, $20 \mathrm{~min}$ ) and the $A_{590}$ value was recorded. Each reaction was repeated in triplicate and the $A_{590}$ values averaged to generate the final value. $A$ standard curve was generated from the standards and this was used to extrapolate the calculated ATP concentrations for the different samples.

\subsection{Assays for the detection of adenylation}

\subsubsection{PPi release assay}

The methods used in this assay were optimised by Owen et al 2010.

PPgreen reagent $A: 4.2 \% \mathrm{w} / \mathrm{v}$ sodium molybdate in $4 \mathrm{M}$ sulfuric acid

PPgreen reagent B: $0.135 \% \mathrm{w} / \mathrm{v}$ brilliant green dye in sterile $d \mathrm{dH}_{2} \mathrm{O}$

Triplicate reactions for each substrate were established in a 96 well plate and in a final volume of $100 \mu \mathrm{L}$ contained: $50 \mathrm{mM}$ Tris- $\mathrm{Cl}$ (pH 7.7), $10 \mathrm{mM} \mathrm{MgCl}$, 1 mM ATP, 5-20 $\mu \mathrm{g}$ enzyme, 0.03 U IP and $1.25 \mathrm{mM}$ amino acid substrate. Plates were then incubated for 30 min at $30^{\circ} \mathrm{C}$, after which reactions were terminated by addition of $20 \mu \mathrm{L}$ PPgreen reagent A per well. Plates were then mixed at $500 \mathrm{rev} / \mathrm{min}$, and $20 \mu \mathrm{L}$ PPgreen reagent green added to each well. Plates were again mixed at $500 \mathrm{rev} / \mathrm{min}$ and $A_{650}$ measured immediately using a microplate reader. 


\subsubsection{Quantification of adenylation by monitoring the consumption of ATP}

For this assay apo-BpsA and the EntF A domain were expressed and then purified in a 50 $\mathrm{mM}$ Tris- $\mathrm{Cl} \mathrm{pH} 7.8$ buffer supplemented with $50 \%$ glycerol $(\mathrm{v} / \mathrm{v})$ to stop the sodium phosphate buffer interfering with the reaction. Triplicate reactions for each substrate were established in a microfuge tube containing the following master mix (MM1); 50 $\mathrm{mM}$ Tris- $\mathrm{Cl} \mathrm{pH} \mathrm{7.8,} 10 \mathrm{mM} \mathrm{MgCl}, 1 \mathrm{mM}$ amino acid, $1 \mathrm{mM}$ ATP, $0.03 \mathrm{U}$ IP and $40 \mu \mathrm{g}$ of enzyme in a final volume of $100 \mu \mathrm{L}$. The reactions were incubated at $30^{\circ} \mathrm{C}, 200 \mathrm{rev} / \mathrm{min}$ for $30 \mathrm{~min} .30 \mu \mathrm{L}$ of MM1 was dispensed into each well of a 96 well plate. A second master mix (MM2) consisting of $50 \mathrm{mM}$ Tris-CL pH 8.5, $10 \mathrm{mM} \mathrm{MgCl}_{2}, 4 \mathrm{mM} \mathrm{L-gln}$ and 2 $\mu \mathrm{M}$ holo-BpsA in a final volume of $20 \mu \mathrm{L}$ was then dispensed in each well to bring about the synthesis of indigoidine. The 96 well plate was then incubated at $200 \mathrm{rev} / \mathrm{min}$ for 30 $\min .200 \mu \mathrm{L}$ of DMSO was then dispensed into each well to solubilise the indigoidine and the plate was shaken at $2000 \mathrm{rev} / \mathrm{min}$ for $10 \mathrm{~min}$. The $A_{590}$ values were then recorded using a PerkinElmer Plate reader.

\subsection{Synthesis and quantification of glutamine analogues}

\subsubsection{Synthesis of indigoidine analogues}

A master mix containing $50 \mathrm{mM}$ Tris- $\mathrm{Cl} \mathrm{pH} 8.5,10 \mathrm{mM} \mathrm{MgCl}$, $10 \mathrm{mM}$ ATP, $10 \mathrm{mM}$ glutamine analogue and $5 \mu \mathrm{M}$ holo-BpsA in a total volume of $400 \mu \mathrm{L}$ was incubated at 25 ${ }^{\circ} \mathrm{C} 200 \mathrm{rev} / \mathrm{min}$ for 16 hours. The master mix was then spun at 13,000 $\mathrm{g}$ for $15 \mathrm{~min}$ and transferred to an Eppendorf centrifugal vacuum concentrator ${ }^{\circledR}$ in which the sample was spun at $45^{\circ} \mathrm{C}$ until the liquid had been evaporated. The indigoidine was then either resuspended in $100 \mu \mathrm{L}$ DMSO or $\mathrm{dd}_{2} \mathrm{O}$ and spun at $13,000 \mathrm{~g}$ for $15 \mathrm{~min}$ to remove insoluble protein and other contaminants and then the supernatant was transferred to a fresh microcentrifuge tube.

\subsubsection{HPLC}

All solvents were HPLC grade. HPLC data was collected on an Agilent 1200 series HPLC. A gradient elution was used from $10-90 \%$ aqueous acetonitrile over 13 min using a 8/4 Nucleodur $\mathrm{C}_{18} \mathrm{HTec}, 5 \mu \mathrm{M}$ HPLC column with a flow rate of $1 \mathrm{ml} / \mathrm{min}$ at $40^{\circ} \mathrm{C} .20 \mu \mathrm{L}$ of sample was injected and detection took place at $590 \mathrm{~nm}$. 


\section{Chapter 3- Development of a high throughput screen to detect inhibitors of PptT from M. tuberculosis and PcpS from $P$. aeruginosa}

\subsection{Published papers}

Jung, J., Bashiri, G., Johnston, J. M., Brown, A. S., Ackerley, D. F., \& Baker, E. N. (2014). Crystal structure of the essential Mycobacterium tuberculosis phosphopantetheinyl transferase PptT, solved as a fusion protein with maltose binding protein. Journal of Structural Biology, 188(3), 274-278. doi:10.1016/j.jsb.2014.10.004

\subsection{Chapter summary}

This chapter details the efforts to develop a high throughput screen to identify inhibitors of PptT, an essential PPTase in M. tuberculosis. The screen relies on a PPTase to attach a phosphopantetheinyl (P-pant) moiety derived from Co-enzyme A (CoA) to the nonribosomal peptide synthetase BpsA, thereby converting it to the active holo form. HoloBpsA can then synthesise indigoidine, an easily detectable blue pigment, from two molecules of L-glutamine in an ATP powered reaction. The rate of indigoidine synthesis is directly proportional to the rate of PPTase-mediated conversion from apo-BpsA to holo-BpsA.

PptT is unstable and almost completely insoluble when expressed in E. coli. An optimised expression and purification scheme was developed to enable high yields. The PptT inhibitor assay parameters were then optimised to increase the sensitivity and reduce the time and reagents necessary for screening. Once this had been completed, a protocol for high throughput inhibitor screening was developed.

Three commercially available drug libraries were screened; The LOPAC ${ }^{1280 \circledast}$, the NIH Diversity Set II and the Spectrum Collection ${ }^{\circledR}$. Additionally, several smaller in-house libraries were also screened. Potential inhibitors were validated and then counter screened against holo-BpsA to ensure they were inhibiting PptT and not BpsA or a 
component of the reaction other than PptT. The top hits were then assayed to identify their $\mathrm{IC}_{50}$ values (the half maximal effective concentration; in this case defined as the concentration of inhibitor that resulted in the maximum rate of indigoidine synthesis in the reaction diminishing by $50 \%$ versus the no-inhibitor control).

The LOPAC ${ }^{1280}$, NIH Diversity Set II and Spectrum Collection ${ }^{\circledR}$ libraries were also screened to find inhibitors of PcpS, an essential PPTase from P. aeruginosa. The top hits identified from these three libraries were then assayed to measure their $\mathrm{IC}_{50}$ values. From the LOPAC $^{1280}$ library, SCH-202676 was identified as an inhibitor in the nanomolar range against PcpS. This compound was then tested in vivo to determine if it had anti-microbial activity against $P$. aeruginosa. While SCH-202676 did not have any discernible antimicrobial activity, it did reduce the production of the NRPS-derived siderophore pyoverdine, indicating that it was having an effect on reducing the level of PcpS activity in vivo.

\subsection{Introduction}

Despite significant investment and efforts by large non-government organisations such as the Bill and Melinda Gates Foundation and World Health Organisation (WHO), tuberculosis (TB) remains one of the most pressing health issues in developing countries. This threat to health has been compounded by the emergence of TB strains that are multi-drug resistant (MDR) and extremely drug resistant (XDR) (World Health Organisation, 2016). In 2015, 1.4 million deaths due to TB were reported, and it continues to be one to the top ten causes of death worldwide (World Health Organisation, 2016).

One of the most difficult aspects of successfully treating TB is the long antibiotic regimes that are needed in order to completely remove the TB infection. Antibiotics need to be administered for several months and this extended treatment time can result in low levels of compliance, which is fuelling the emergence of MDR and XDR strains of TB. There is a pressing need to identify novel compounds which can target the slow growing or non-replicating $M$. tuberculosis bacilli. These compounds have the potential to significantly shorten the current treatment times and will hopefully be effective against XDR and MDR strains of TB (Uplekar et al., 2015). 
A potential novel drug target is the PPTase super family. Like many bacteria, $M$. tuberculosis contains two PPTases; a Type I PPTase called AcpS whose structure, function and potential as a drug target is discussed in-depth in Section 4.2. The second PPTase is a Type II PPTase called PptT which is responsible for the post translational modification of a diverse range of enzymes involved in both primary and secondary biosynthesis (Chalut et al., 2006; Leblanc et al., 2012).

\subsubsection{Function and the essential nature of PptT}

One of the hallmarks of $M$. tuberculosis is the diversity and complexity of the bacterium's cell wall, which includes a range of uncommon fatty acids, glycolipids and polyketide natural products. The constituents of the cell wall help M. tuberculosis evade and counteract the host's immune system (Cole et al., 1998; Neyrolles and Guilhot, 2011). In order to synthesise this diverse group of membrane components, $M$. tuberculosis contains at least 18 PKSs and two FAS systems (Chalut et al., 2006; Neyrolles and Guilhot, 2011). PptT post-translationally modifies the PKSs involved in fatty acid synthesis and secondary metabolite production, and it has recently been shown to modify AcpM, the carrier protein from the FAS II system (Zimhony et al., 2015). This is unusual, as in most characterised bacteria it is the Type I PPTase AcpS that activates this protein, and indeed, E. coli AcpS is capable of attaching a Ppant motif to the $M$. tuberculosis AcpM (part of the FAS-II system) (Zimhony et al., 2015). M. tuberculosis also contains several NRPSs and PKSs involved in the production of mycobactin, an iron chelating siderophore (Sritharan, 2016), all of which are activated by PptT. Targeting iron acquisition in M. tuberculosis is increasingly being explored as a promising antibiotic therapy (Lamb, 2015).

PptT (Rv2794c) was originally identified as being essential in a pioneering genome wide transposon mutant library screening undertaken by Sassetti et al. (2003). Confirmation of PptT being essential for mycobacterial growth in vitro was provided by Chalut et al. (2006) who confirmed the essential nature of the PptT homolog in M. smegmatis and $M$. bovis, two related Mycobacterium species. A pptT knockout mutant was generated that was only viable when the $M$. smegmatis strain was complemented with the pptT gene on a thermosensitive plasmid. When the $\Delta$ pptT M. smegmatis strain was grown at a 
non-permissive temperature for the plasmid the strain was non-viable, indicating that in M. smegmatis PptT is essential. A similar experiment was also repeated in M. bovis BCG, a strain of bacteria much more closely related to $M$. tuberculosis. The $p p t T$ homolog was knocked out and a plasmid harbouring pptT under the control of the TetR-controlled gene system was used to complement the mutant $M$. bovis strain. The strain was viable when tetracycline was present in the growth media but was unable to replicate when tetracycline wasn't present, showing that PptT was also essential in the M. bovis strain (Chalut et al., 2006). These two experiments provide strong evidence that PptT is also essential in M. tuberculosis.

Further work by Leblanc et al (2012) characterised the essential nature of PptT in vivo. A M. tuberculosis $\Delta p p t T$ mutant complemented by a copy of $p p t T$ on a plasmid under the control of a tetracycline inducible promoter was created in the same manner as the previously described $M$. bovis $\triangle p p t T$ mutant. Leblanc et al then showed that the mutant was only viable in murine macrophages and mice in the presence of tetracycline or a tetracycline analogue, indicating that PptT is essential for in vivo growth. Some effort was also made to characterise the level of inhibition of PptT required to have a bactericidal effect. A western blot of the PptT knockout mutant in $M$. bovis across a range of tetracycline concentrations showed that at least $95 \%$ inhibition was necessary for the complete inhibition of growth (Leblanc et al., 2012). However, Leblanc et al did not report a percent inhibition value for the PptT deficient $M$. tuberculosis mutant.

\subsubsection{Structure of PptT}

Two papers have been published reporting the crystal structure of PptT (Jung et al., 2014; Vickery et al., 2014), one led by our University of Auckland collaborator Professor Ted Baker, with our assistance. Due to the poor solubility and stability of PptT, both structures were solved as fusion proteins with maltose binding protein. PptT is 227 amino acids in length and almost completely insoluble as a $6 \mathrm{His}$ tagged protein. In order to improve solubility, it was fused to MBP which enabled the crystal structure to be solved at a resolution of $1.75 \AA$. PptT is comprised of two $\alpha / \beta$ domains having a pseudo 2-fold symmetry as shown in Fig. 3.1A. PptT is structurally similar to other type II PPTases that have been crystallised (Sfp from B. subtilis and Hs-Ppt from H. sapiens) 
(Reuter et al., 1999). Despite sharing the same fold there are important differences. The binding pocket for CoA in PptT is different to both Sfp and Hs-Ppt. The CoA molecule displays a $U$ shape confirmation (Fig. 3.1B) and the phosphopantetheine arm extends into a deep hydrophobic pocket. Comparatively, the phosphopantetheine arm in both Sfp and Hs-Ppt is much more exposed (Jung et al., 2014).
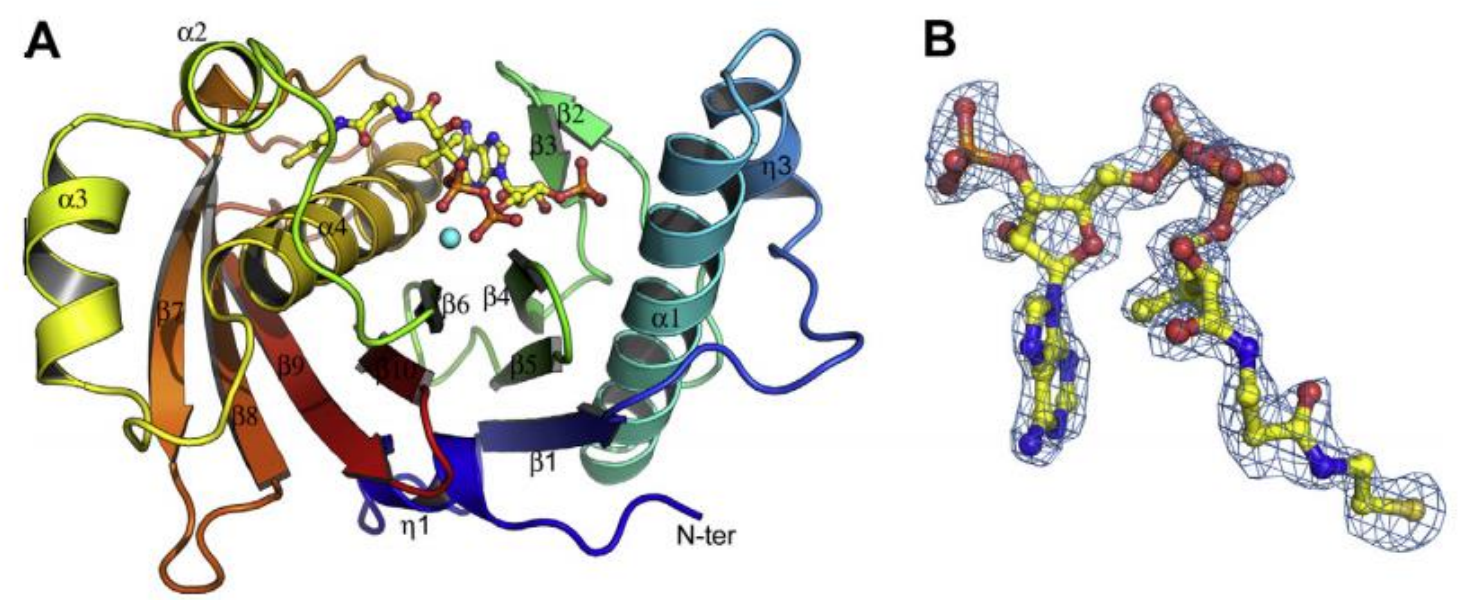

Figure 3.1 Crystal structure of PptT: (A) A cartoon ribbon diagram of PptT with CoA bound illustrating the structure. (B) The U-shaped confirmation of CoA within the PptT binding pocket is displayed. This figure is adapted with permission from our co-authored manuscript (Jung et al., 2014).

The structural differences within the PptT binding pocket and orientation of CoA are important as previously published screens for Sfp and AcpS inhibitors have relied on CoA analogues for the detection of inhibition (Duckworth and Aldrich, 2010; Foley et al., 2014; Yasgar et al., 2010). Work by Leblanc et al showed that PptT was unable to attach fluorescent CoA analogues (CoA488 and CoA547) to carrier proteins (Leblanc et al., 2012). This result however is contradicted by Vickery et al (2014), which reports use of a CoA fluorescence based assay to show that Sfp inhibitors also had an inhibitory effect on PptT. Interestingly, although they could detect inhibition using rhodamine CoA, Vickery et al could not calculate a $\mathrm{K}_{d}$ with rhodamine CoA and PptT, providing further evidence that PptT is highly selective for specific forms of CoA. They hypothesised that the rhodamine CoA was not adopting the $U$-shaped binding conformation and instead the pantetheine arm is extending out of the binding pocket (Vickery et al., 2014). Importantly, the BpsA assay that is described in this chapter uses the native CoA, which ensures that substrate specificity will not be an issue. 
Importantly, there are also low levels of residue conservation in the active site of PptT when compared to Hs-Ppt along with several differences in the structure (Jung et al., 2014). These differences provide hope that it might be possible to identify selective PptT inhibitors that do not have an inhibitory effect on Hs-Ppt, as such an effect would potentially be a source of significant toxicity to human cells.

\subsubsection{Published screens for the detection of PptT}

Several High Throughput Screens (HTS) for Sfp inhibitors have been developed (as discussed in-depth in Section 1.7). A HTS screen for PptT inhibitors has yet to be published, which could in part be due to the difficulty in using PptT in assays (due to its poor solubility and stability), or that it doesn't accept the CoA analogues that previous Sfp screens have relied upon. However, two proof of principle assays to detect the inhibition of PptT have been published in papers that have either focused on the structure of PptT or on characterising the enzyme in vivo.

The first published screen was developed by Leblanc et al in 2012. A Scintillation Proximity Assay (SPA) using radiolabelled CoA was developed, expanding on their previous research showing that CoA-fluorescent dye conjugates were not accepted by PptT. The radiolabelled assay works by using microspheres (SPA beads) that have a biotinylated ACP domain bound to the beads. Similar to a FRET assay, the beads emit light when a radiolabelled molecule is bound or close by (Leblanc et al., 2012). When PptT attaches the radiolabelled CoA to the ACP domain and converts it to holo-ACP, the increase in light can be detected. In a control experiment increasing the amount of PptT present in the assay, it was seen that the level of light emitted increased. Leblanc stated that the assay was effective for identifying inhibitors of PptT but did not provide any data, nor did they show that it could be used to detect the inhibition of PptT by any compounds showing broad inhibition of PPTases (Leblanc et al., 2012).

A second assay to detect inhibitors of PptT was published by Vickery et al as part of their paper on the structure of PptT in 2014. They used a fluorescence polarization assay based on rhodamine-CoA, a fluorescent CoA analogue, and two different carrier protein substrates, VibB from $V$. cholerae and MAS, a polyketide synthetase from $M$. tuberculosis, a native substrate of PptT. A panel of known Sfp inhibitors were screened, 
which had a variety of different $\mathrm{IC}_{50}$ values. The inhibitor $\mathrm{SCH}-202676$ gave the highest level of inhibition, with a reported $\mathrm{IC}_{50}$ of $0.5 \mu \mathrm{M}$ against MAS and $0.8 \mu \mathrm{M}$ against VibB. However, large errors were reported when measuring the $\mathrm{IC}_{50}$ values of $\mathrm{SCH}-202676$ (Vickery et al., 2014). Interestingly, several of the inhibitors had different IC 50 values for both MAS and VibB. This could be due to several of the compounds inhibiting the carrier protein as well as the PPTase. For example, 6-NOBP which had an $\mathrm{IC}_{50}$ of $24 \pm 2 \mu \mathrm{M}$ with MAS and $17 \pm 2 \mu \mathrm{M}$ with VibB has been shown to also inhibit BpsA, albeit at a lower level compared to the PPTase (Owen et al., 2011).

\subsubsection{Inhibition of PcpS from $P$. aeruginosa}

Pseudomonas aeruginosa is an opportunistic pathogen mainly found in hospitals and areas where there are high numbers of people with compromised immune systems, for example among burn victims or HIV patients. P. aeruginosa is extremely difficult to treat, in part because it has several multi-drug efflux pumps that allow it to pump out a wide range of antibiotics. It also readily forms biofilms in infected areas. Worryingly, $P$. aeruginosa is also becoming increasingly resistant to antibiotics such as the carbapenem family that are currently used in front-line treatment. Multi-drug resistant and even panresistant drug strains (resistant to all treatments) are emerging. These strains are currently impossible to treat and have an extremely high mortality rate (Kerr and Snelling, 2009). Consequently, there is a pressing need to identify new drug targets in $P$. aeruginosa. The WHO published a priority pathogen list for the R\&D of new antibiotics in 2017 and carbapenem-resistant $P$. aeruginosa is listed as the second most critical priority.

In contrast to most other bacterial species, P. aeruginosa possesses only one PPTase. PcpS is a Type II PPTase with broad substrate specificity that post-translationally modifies enzymes involved in both primary and secondary biosynthesis. PcpS has been examined in less detail than PptT as a drug target. A paper published by Barekzi et al (2004) has shown that PcpS is essential. To demonstrate this, Barekzi et al developed a strain of $P$. aeruginosa that has the $E$. coli PPTase acpS chromosomally integrated. Only in the strain with the chromosomally integrated acpS was it possible to create a $\Delta p c p S$ mutant. This confirmed that PcpS is necessary for primary metabolism as the 
recombinant mutant could not function without a Type I PPTase to enable activation of FAS enzymes in the absence of PcpS (Barekzi et al., 2004).

\subsubsection{Detection of PPTase inhibition using BpsA}

In a 2011 paper from our lab, Owen et al showed that it was possible to use the indigoidine synthesising non-ribosomal peptide synthetase BpsA to derive the kinetic parameters of PPTases by monitoring the rate of indigoidine synthesis (Owen et al., 2011). To achieve this, BpsA is purified in the inactive apo form, after which it needs to be post-translationally modified by a PPTase in order to be functional (Fig. 3.2). The rate of indigoidine synthesis acts as a surrogate for the level of PPTase activity as it is a measure of the conversion of apo-BpsA to holo-BpsA. Importantly, holo-BpsA can be used to counter screen potential hits to ensure they are inhibiting PptT rather than another component of the reaction.

Owen et al (2011) completed a proof of principal experiment by evaluating PPTases for inhibition with 6-NOBP, a known Sfp inhibitor. PcpS from $P$. aeruginosa, Sfp from $B$. subtilis and PP1183 from P. putida were all tested, and all three PPTases were inhibited by 6-NOBP. The level of inhibition was reduced when the concentration of CoA was increased from $2.5 \mu \mathrm{M}$ to $10 \mu \mathrm{M}$. Based on the success of these pilot studies it was reasoned that it should be possible to develop a HTS for inhibitors of PptT using BpsA. 


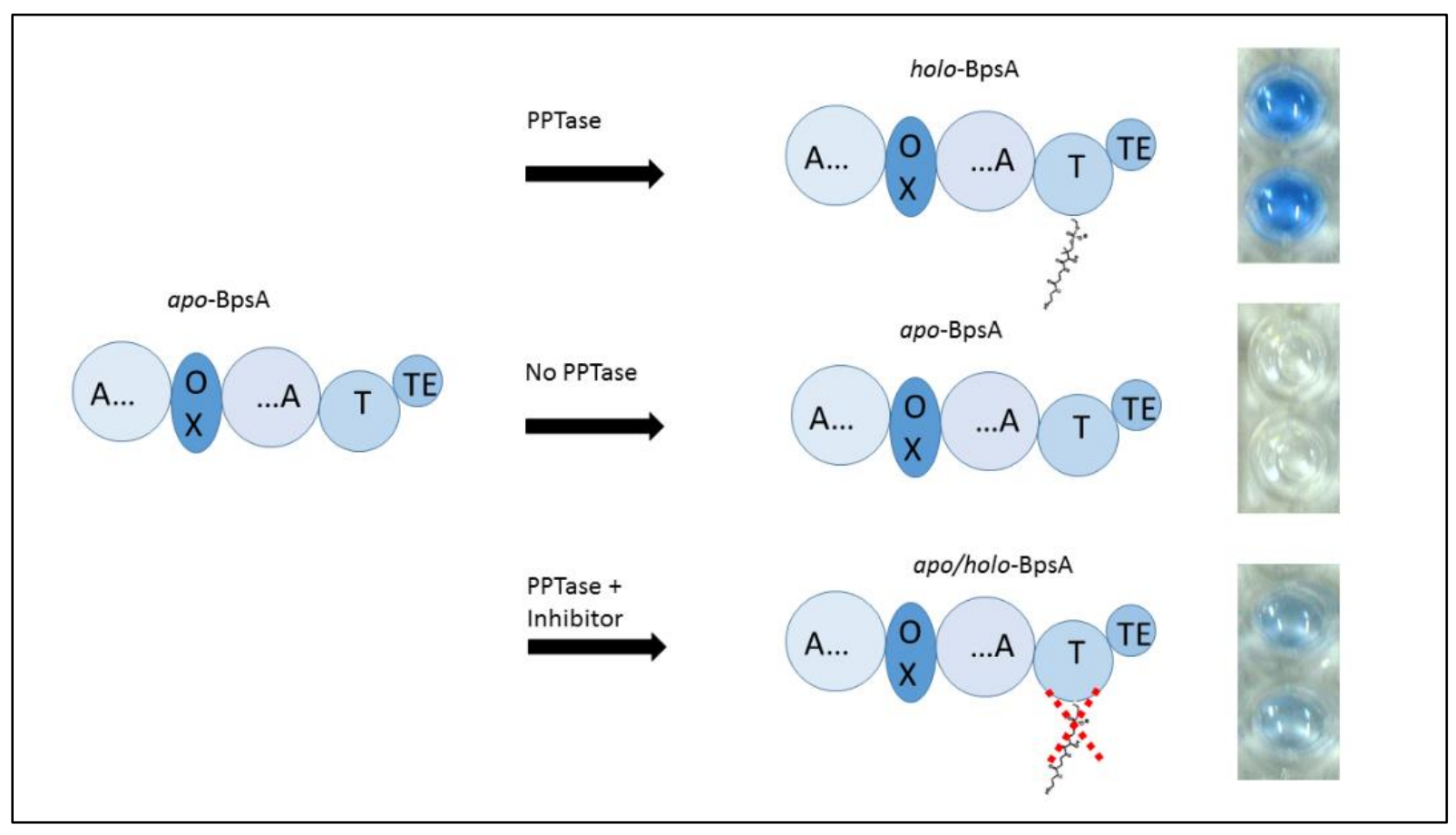

Figure 3.2 Schematic diagram illustrating BpsA being used to detect inhibition: When apo-BpsA is posttranslationally modified by a PPTase and converted to holo-BpsA it is able to synthesise the blue pigment indigoidine. If a PPTase and an inhibitor are added together the rate of indigoidine synthesis is lower as the amount of BpsA converted to the holo form is reduced. This means that the level of indigoidine synthesised is a direct reporter for PPTase inhibition.

\subsubsection{Screening for PcpS inhibitors using BpsA}

As part of my Honours thesis I optimised a HTS protocol for the detection of inhibitors of PcpS from $P$. aeruginosa and completed a proof of principle screen of a commercially available drug library. Owen et al showed that PcpS could convert BpsA into the active holo form more efficiently than Sfp (i.e., with a higher $k_{c a t} / K_{m}$ ), indicating that it would be a good candidate for HTS using BpsA. The screening protocol developed was similar to PptT which is discussed in detail in Section 3.6, but had the following modifications; the screening master-mix contained $50 \mathrm{mM}$ Tris- $\mathrm{Cl} \mathrm{pH} \mathrm{7.8,} 10 \mathrm{mM} \mathrm{MgCl}_{2} 2.5 \mathrm{mM}$ ATP, 4 $\mathrm{mM}$ L-glutamine and 0.8 $\mu \mathrm{M}$ BpsA (all values are displayed as final concentrations). To initiate the reaction PcpS was added to each well at a concentration of $0.18 \mu \mathrm{M}$ in $20 \mu \mathrm{L}$ of $\mathrm{dd}_{2} \mathrm{O}$. Since PcpS is more efficient at modifying BpsA, less enzyme was needed for each assay. 


\subsubsection{LOPAC $^{1280}$ screen for inhibitors of PcpS}

The LOPAC ${ }^{1280}$ (Library of Pharmacologically Active Compounds) is a commercially available drug library produced by Sigma Aldrich. The library contains 1,280 bioactive small molecules arrayed across sixteen 96 well plates. The compounds are a mix of inhibitors, receptor ligands, pharma-developed tools and improved drugs, with over $50 \%$ of the compounds known to exert an effect on neurotransmission. The compounds are dissolved in DMSO and provided at a working concentration of $1 \mathrm{mM}$.

In my Honours research, this library proved to be a rich source of novel PcpS inhibitors, with a wide range of compounds found to have inhibitory activity. In total, 14 compounds were found to have a range of inhibitory activities against PcpS after counter-screening against holo-BpsA had been completed. The top seven inhibitors are displayed below, all except lansoprazole displayed complete inhibition of indigoidine synthesis at a concentration of $20 \mu \mathrm{M}$. Interestingly, they all appeared to be broad spectrum inhibitors of PPTases with all of them also being able to inhibit Sfp to varying degrees. 


\begin{tabular}{|c|c|c|c|}
\hline Name & Structure & $\begin{array}{c}\text { Level of } \\
\text { inhibition }\end{array}$ & Bioactivity $^{1}$ \\
\hline Bay 11-7085 & & $\begin{array}{c}\text { Complete at } 20 \\
\mu \mathrm{M}\end{array}$ & $\begin{array}{l}\text { Active against } \\
\text { Sfp and PptT }\end{array}$ \\
\hline Lansoprazole & & $75 \%$ at $20 \mu \mathrm{M}$ & $\begin{array}{l}\text { Active against } \\
\text { Sfp and PptT }\end{array}$ \\
\hline PD 404,182 & & $\begin{array}{c}\text { Complete at } 20 \\
\mu \mathrm{M}\end{array}$ & $\begin{array}{l}\text { Active against } \\
\text { Sfp and PptT }\end{array}$ \\
\hline $\begin{array}{l}\text { SCH-202676 } \\
\text { hydrobromide }\end{array}$ & & $\begin{array}{c}\text { Complete at } 20 \\
\mu \mathrm{M}\end{array}$ & $\begin{array}{c}\text { Active against } \\
\text { Sfp and PptT }\end{array}$ \\
\hline $\begin{array}{c}\text { Sanguinarine } \\
\text { chloride }\end{array}$ & & $\begin{array}{c}\text { Complete at } 20 \\
\mu \mathrm{M}\end{array}$ & $\begin{array}{l}\text { Active against } \\
\text { Sfp and PptT }\end{array}$ \\
\hline Ebselen & & $\begin{array}{c}\text { Complete at } 20 \\
\mu \mathrm{M}\end{array}$ & $\begin{array}{c}\text { Active against } \\
\text { Sfp and PptT }\end{array}$ \\
\hline 6-NOBP & & $\begin{array}{c}\text { Complete at } 20 \\
\mu \mathrm{M}\end{array}$ & $\begin{array}{l}\text { Active against } \\
\text { Sfp and PptT }\end{array}$ \\
\hline
\end{tabular}

Table 3.1 Top inhibitors identified from the LOPAC ${ }^{1280}$ screen: Structures are taken from the PubChem compound page and bioassay data is taken from the PubChem. ${ }^{1}$ Bioassay database available at: https://pubchem.ncbi.nlm.nih.gov/bioassay/1490 for Sfp.

This pilot study showed that it was possible to identify PPTase inhibitors using the BpsA assay in a HTS and with further optimisation it should be possible to adapt the screen for different PPTases. The screening of additional libraries for PcpS inhibitors and characterisation of the inhibitors listed here are discussed in Section 3.10. 


\subsection{Research aims}

- Develop an optimised protocol for the expression and purification of PptT

- Develop and optimise a screen to detect the inhibition of PptT

- Screen the LOPAC ${ }^{1280}$ and NIH Diversity Set II and Spectrum Collection to identify inhibitors of PptT and PcpS

- Determine the $\mathrm{IC}_{50}$ values of the top inhibitors.

- Evaluate the in vivo efficacy of the top inhibitors

\subsection{Cloning, expression and purification of PptT}

The pptT gene was cloned into the pET28a(+)expression vector via PCR amplification from genomic DNA from the M. tuberculosis strain H37Ra. The routine cloning is fully described in Section 2.6. The construct was sequenced verified to confirm that no point mutations had been introduced and that the $6 \mathrm{His}$ purification tag was intact. The construct was then transformed into E. coli strain BL21 (DE3), which is optimised for protein expression and purification using the method described in Section 2.4.6.4.

SDS-PAGE analysis of the initial protein expression and purification indicated that PptT was almost completely insoluble and performed poorly throughout the various purification steps. The poor solubility and purification has been encountered by other groups working with PptT (Jung et al., 2014; Rottier et al., 2013; Vickery et al., 2014).

To try and improve the purification protocol by introducing increased levels of glycerol to the purification buffers (as described in Section 2.5) was not effective. A range of techniques that have been found to improve protein over-expression and solubility by Oganesyan et al., (2007) including; betaine and sorbitol, varying expression temperatures and heat shocking the culture at $42^{\circ} \mathrm{C}$ also did not substantially improve yields or solubility (Oganesyan et al., 2007). One L of culture expressing PptT typically resulted in yields of approximately $0.8-1.6 \mathrm{mg}$ of purified PptT under all conditions tested. The purified PptT was also unstable and rapidly aggregated when it was thawed from $-80^{\circ} \mathrm{C}$, making it difficult to use in kinetic assays.

The low in vitro solubility of PptT did not correspond to the in vivo performance in $E$. coli. When PptT was co-expressed with BpsA in E. coli PptT was capable of rapidly 
converting BpsA to the active holo form as evidenced by the clear detection of indigoidine production on plates (Fig. 3.4A). The reason for this inconsistency was clarified when Rottier et al (2013) showed that PptT is dependent on both CoA and $\mathrm{MgCl}_{2}$ for stability. The addition of $\mathrm{CoA}$ at a concentration of $50 \mu \mathrm{M}$ and $\mathrm{MgCl}_{2}$ at a concentration of $10 \mathrm{mM}$ to the lysis and purification steps, Rottier et al were able to significantly improve the solubility and reduce the formation of aggregation bodies when PptT was purified (Rottier et al., 2013).

A modified purification protocol based on the method described in Section 2.5.5.2 was developed. $\mathrm{CoA}$ and $\mathrm{MgCl}_{2}$ at concentrations of $50 \mu \mathrm{M}$ and $10 \mathrm{mM}$ respectively were added to the bind buffer, in which cell pellets were resuspended in prior to lysis. This improved purification protocol resulted in significantly higher yields, typically 3.0-3.5 $\mathrm{mg} / \mathrm{ml}$ of PptT from a $400 \mathrm{ml}$ culture volume. An SDS-PAGE gel from the optimised purification process is shown in Fig. 3.3. In addition to the higher yields, the stability and activity of the PptT was improved over the standard PPTase purification method.

An alternative purification method published in 2015 by Zimhony et al also improved the solubility of PptT. The authors stated that they saw improved solubility and purification yields by supplementing the purification buffers with $0.25 \mathrm{M} \mathrm{L-arginine} \mathrm{(Zimhony} \mathrm{et} \mathrm{al.,}$ 2015), a well-known additive that can improve protein stability and reduce aggregation (Arakawa et al., 2007). However, no experimental evidence was provided and their published method did not result in improved yields when attempted in our laboratory, so L-arginine supplementation was not used in our ongoing purification protocols. 


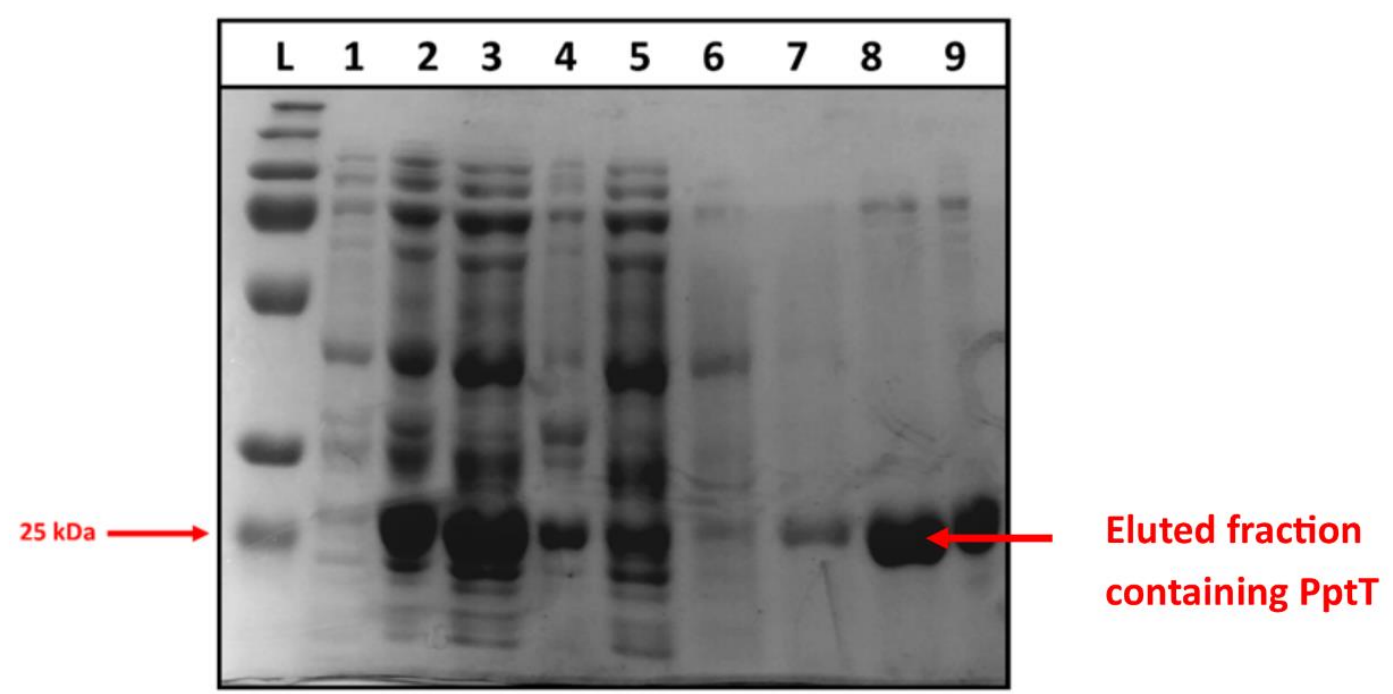

Figure 3.3 A 12\% SDS-PAGE gel illustrating the improved solubility of PptT using optimised purification protocol: The $25 \mathrm{kDa}$ band in the marker (column L) is indicated by the red arrow. PptT is approximately $24.7 \mathrm{kDa}$. PptT is strongly induced in E. coli cells as illustrated by the strong band in column 3 which was sampled 24 hours post induction. The majority of PptT in the E. coli cells is soluble (column 3). The labels above the gel correspond to the following: L) Molecular weight ladder 1) Pre-induction sample 2) Twenty four hour post-induction sample 3) Soluble fraction following lysis 4) Insoluble fraction following lysis 5) Elution collected after sample applied to the column 6) Elution collected after bind buffer was applied to the column 7) Elution collected after wash buffer was applied to the column 8) Elution collected after elution buffer was applied to the column. 9) Sample after desalting. Twelve $\mu \mathrm{L}$ of sample, resuspended in 3 X SDS loading buffer was added to each lane.

\subsection{Analysis of PptT activity with BpsA in vivo and in vitro}

In order for BpsA to be capable of detecting inhibitors of PptT it was first necessary to ascertain whether BpsA is an effective substrate for PptT. Previous work by Owen et al had shown that BpsA is a substrate for a wide range of PPTases (Owen et al., 2012) and since PptT post-translationally modifies both NRPSs and PKSs in M. tuberculosis I reasoned that it was likely to possess a relatively high level of substrate promiscuity. To test whether BpsA was a substrate of PptT it was first tested in vivo. BpsA and PptT were both expressed using complementary plasmids in a strain of $E$. coli in which the endogenous non-essential type II PPTase entD had been knocked out. The $\Delta$ entD strain is incapable of converting BpsA to the active holo form as the other endogenous (Type I) E. coli PPTase AcpS does not recognise BpsA efficiently enough to allow the detection of pigment in vivo. When PptT was co-expressed with BpsA the colonies rapidly turned blue indicating that BpsA was a substrate for PptT (Fig 3.4A). To quantify the level of activity between PptT and BpsA, indigoidine synthesis was then assayed in vitro. 
PptT was benchmarked against Sfp, often considered the "gold standard" of the PPTase field (Beld et al., 2014), to determine if it exhibited similar levels of catalytic activity and therefore was a good candidate for high throughput screening to identify inhibitors. It was important to confirm that PptT was able to activate BpsA to a detectable level and within a short enough time frame to make screening for PptT inhibitors viable. It was reasoned that ideally a measurable level of detection would occur within $15 \mathrm{~min}$. This would allow the screening of up to four compound library plates per hour.

Sfp was expressed and purified as described in Section 2.5.5.2. Sfp is soluble enough to not need $\mathrm{MgCl}_{2}$ and $\mathrm{CoA}$ supplementation in the binding buffers. A kinetic assay was established as described in methods Section 2.7.1 to determine conversion rate of BpsA into the holo form by PptT. Both PptT and Sfp were assayed at concentrations of $0.2 \mu \mathrm{M}$ and at $\mathrm{pH}$ of 8.0. PptT is substantially slower than Sfp at post-translationally modifying BpsA (Fig. 3.4B). Despite PptT being catalytically slower than Sfp, I considered that the rate of conversion of BpsA was rapid enough to facilitate the HTS of PptT to detect inhibitors, particularly if the reaction parameters could be further optimised.

A

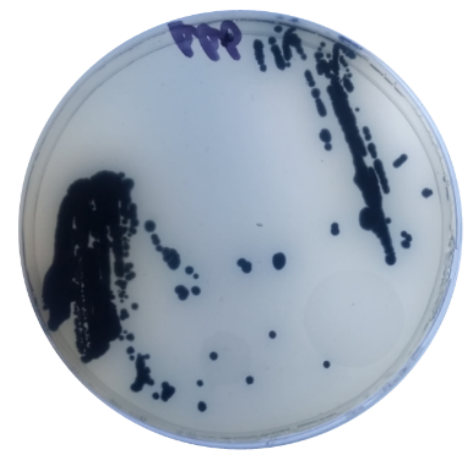

B

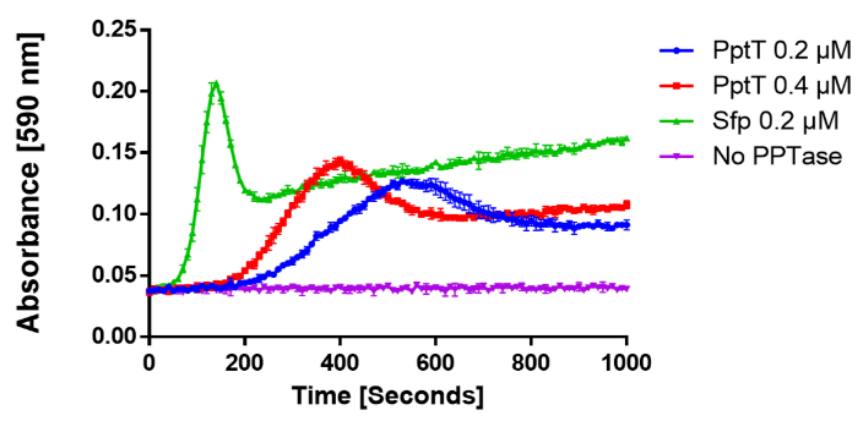

Figure 3.4 Activity of PptT with BpsA both in vivo and in vitro: (A) PptT can convert BpsA to the active holo form in vivo. BpsA and PptT were co-expressed on plasmids with IPTG inducible expression in an $E$. coli $\Delta$ entD strain incapable of post-translationally modifying BpsA unless a PPTase is co-expressed. The bacterium was plated on pigment producing plates and expression of BpsA and PptT was induced using IPTG, the photo was taken 8 hours after induction and shows indigoidine production. (B) While BpsA can be converted into the holo form by PptT (blue and red lines) in vitro it is slower than Sfp (green line). Data is the average of three replicates with error bars representing \pm SEM. 


\subsection{Development and optimisation of an assay to detect PptT inhibition}

In order to develop an assay to precisely quantify the level of inhibition of PptT based on the conversion of apo-BpsA to holo-BpsA, several factors needed to be taken into consideration. First, it was desirable that the reaction be optimised to improve the signal intensity and reduce the amount of enzyme needed and time taken. Once the reaction had been optimised it was then essential to confirm that different levels of PptT inhibition could be detected under high throughput screening conditions. Finally, a work flow needed to be developed which would allow the detection, validation and counter screening of PptT inhibitors from commercial drug libraries.

\subsubsection{Optimisation of reaction parameters}

The goal of the assay optimisation was threefold. First, to reduce the amount of enzyme needed in the screen as the expression and purification of PptT and BpsA was a major bottleneck in the assay. Second, I sought to reduce the time taken for the assay to detect PptT inhibition and thereby increase the overall speed of the reaction. Finally, the reaction had to be sensitive enough to allow the quantification of PptT inhibition by compounds having a range of inhibition profiles.

\subsubsection{Optimisation of $\mathrm{pH}$}

It was necessary to take into account the effect of $\mathrm{pH}$ on three different reaction parameters; the optimal pH for PptT activity, the optimal pH for BpsA activity and the optimal pH for indigoidine stability. Leblanc et al tested the activity of PptT at pH 7.0 and 8.0 and found it had more activity at pH 8.0 (Leblanc et al., 2012). The optimal pH for holo-BpsA is between 8.5 and 8.9 in a Tris-HCl buffer (Owen et al., 2011). However, the stability of indigoidine is also affected by $\mathrm{pH}$ as shown in (Owen, 2010), with indigoidine being the most stable in a $\mathrm{pH}$ range of $6.6-7.0$ compared to the more alkaline buffer conditions preferred by the enzymes. The optimal $\mathrm{pH}$ of indigoidine was deemed to be less important than the enzymes as I anticipated that we would be measuring the reaction rate rather than the maximal $A_{590}$ value. Therefore, a $\mathrm{pH}$ range from 7.8 to 8.4 was assayed to determine the optimal $\mathrm{pH}$. Eighty $\mu \mathrm{L}$ of a reaction master mix with a final 
concentration of $50 \mathrm{mM}$ Tris- $\mathrm{HCl}$ (at a range of $\mathrm{pH}$ ), $10 \mathrm{mM} \mathrm{MgCl}, 6 \mathrm{mM} \mathrm{L-gln,} 6 \mathrm{mM}$ ATP, $5 \mu \mathrm{M}$ CoA and $2 \mu \mathrm{M}$ of BpsA) was added to each well. The reaction was initiated by the addition of $0.4 \mu \mathrm{M}$ PptT in $20 \mu \mathrm{L}$ of $\mathrm{dd} \mathrm{H}_{2} \mathrm{O}$. The 96 well plate was shaken at 1000 $\mathrm{rev} / \mathrm{min}$ for $10 \mathrm{~s}$ to ensure all reaction components were mixed and $A_{590}$ values were recorded every $10 \mathrm{~s}$ for $800 \mathrm{~s}$.

The average values of three repeats are plotted in Fig. 3.5A. Modifying the $\mathrm{pH}$ was found to not have a major effect on either the rate of indigoidine synthesis or the maximal $A_{590}$ values. It is possible that as the catalytic efficacy of PptT decreased at higher $\mathrm{pH}$, slowing down the conversion of BpsA to the holo form, the rate of indigoidine synthesis from holo-BpsA was increasing to counteract this. From this data, a pH of 8.0 was chosen to run the assays, reasoning that the achieving the optimal pH for PptT was likely to be most important.

\subsubsection{Optimisation of enzyme concentration}

The optimal concentration of PptT was determined by assaying three different concentrations of PptT $(0.2 \mu \mathrm{M}$ to $0.8 \mu \mathrm{M})$. The previous assay comparing the activity of PptT to Sfp (Fig. 3.4B) had shown that $0.2 \mu \mathrm{M}$ resulted in a reduced reaction speed that would make HTS difficult. Conversely if the concentration was increased significantly (to $1 \mu \mathrm{M}$ or above) it would be time consuming to purify the amount of PptT required for high-throughput assays. Additionally, it was considered that increasing the concentration of PptT would reduce the sensitivity of the assay as a higher concentration of inhibitor would also be needed. To determine the optimal PptT concentration, $80 \mu \mathrm{L}$ of a master mix (50mM Tris-Cl, $10 \mathrm{mM} \mathrm{MgCl}$, $6 \mathrm{mM} \mathrm{L-Gln}, 6 \mathrm{mM}$ ATP, $5 \mu \mathrm{M}$ CoA and $2 \mu \mathrm{M}$ of BpsA) was added to individual wells of a 96 well plate. To initiate the reaction, $\mathrm{PptT}$ at a range of concentrations in a final volume of $20 \mu \mathrm{L}$ was added to each well. The 96 well plate was shaken at $1000 \mathrm{rev} / \mathrm{min}$ for $10 \mathrm{~s}$ to ensure all reaction components were mixed and $A_{590}$ values were recorded every $10 \mathrm{~s}$. The average values of three repeats are plotted in Fig. 3.5B. As expected, it was found that increasing the concentration of PptT resulted in an increased rate of indigoidine synthesis. An optimal concentration of PptT was selected as being $0.4 \mu \mathrm{M}$, to strike a balance between 
overall reaction time, reaction sensitivity and amount of time required to purify additional PptT.

While not as critical as the concentration of PptT, reducing the concentration of BpsA present in each assay would allow more assays to be ran per purification cycle. To determine if this was viable, a master mix $(50 \mathrm{mM}$ Tris- $\mathrm{Cl}, 10 \mathrm{mM} \mathrm{MgCl}$, $6 \mathrm{mM} \mathrm{L-Gln}, 6$ $\mathrm{mM}$ ATP, $5 \mu \mathrm{M}$ CoA in a final volume of $80 \mu \mathrm{L}$ ) with a range of BpsA concentrations from $0.5-2 \mu \mathrm{M}$ was added to individual wells of a 96 well plate and the reaction in each well was initiated by the addition of $0.4 \mu \mathrm{M}$ PptT in $20 \mu \mathrm{L}$ of $d_{d H_{2}} \mathrm{O}$. The 96 well plate was shaken at $1000 \mathrm{rev} / \mathrm{min}$ for $10 \mathrm{~s}$ to ensure all reaction components were mixed and $A_{590}$ values were recorded every $10 \mathrm{~s}$. The average values of three repeats are plotted in Fig. 3.5C.

Lowering the concentration of BpsA resulted in a reduced reaction rate. While it was still possible to detect the conversion of BpsA into the holo form, with lower concentrations of BpsA the increased time it takes to reach the maximum $\mathrm{A}_{590}$ and reduced reaction rate were deemed too detrimental to the overall time and sensitivity of the assay to lower the concentration of BpsA to below $2 \mu \mathrm{M}$.

Another important consideration for the assay optimisation was the concentration of CoA. Owen et al (2011) had shown that increasing the concentration of CoA increased the reaction rate. However, it also had to be taken into account that increasing the CoA concentration also effectively dilutes a competitive inhibitor, resulting in the overall sensitivity of the assay being reduced. Unlike the assays performed in by Owen et al (2011), PptT was co-purified with CoA, meaning that there would be a base level of activity without any additional CoA being added. If all of the PptT present in the reaction already had CoA bound into the active site then up to $20 \%$ of the BpsA might be activated before a competitive inhibitor could gain access to the substrate binding pocket.

To determine the optimal amount of $\mathrm{CoA}$ to add a master mix $(50 \mathrm{mM}$ Tris- $\mathrm{Cl}, 10 \mathrm{mM}$ $\mathrm{MgCl}_{2}, 6 \mathrm{mM} \mathrm{L}-\mathrm{Gln}, 6 \mathrm{mM}$ ATP, $2 \mu \mathrm{M}$ BpsA in a final volume of $80 \mu \mathrm{L}$ ) containing a variable amount of CoA was set up. The master mix was then added to a 96 well plate and the reaction was initiated by the addition of $0.4 \mu \mathrm{M}$ PptT in $20 \mu \mathrm{L}$ of $\mathrm{ddH}_{2} \mathrm{O}$. The 96 
well plate was shaken at $1000 \mathrm{rev} / \mathrm{min}$ for $10 \mathrm{~s}$ to ensure all reaction components were mixed and $A_{590}$ values were recorded every $10 \mathrm{~s}$. The average values of three repeats are plotted in Fig. 3.5D. Adding additional CoA increased the reaction rate up to a concentration of $2.5 \mu \mathrm{M}$. A CoA concentration of $5 \mu \mathrm{M}$ was used in the optimised protocol, at this concentration of $\mathrm{CoA}$ the rapid conversion of BpsA from the apo to holo form occurred.

A

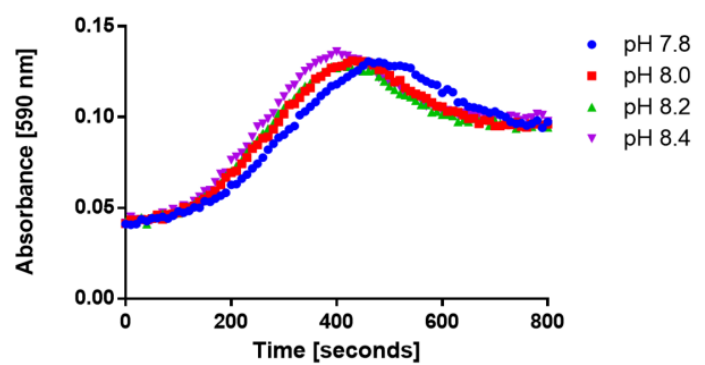

C

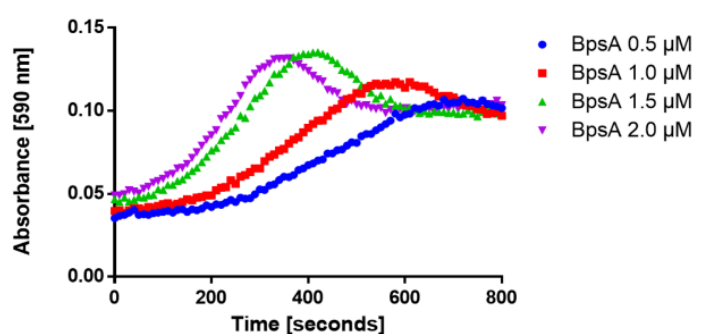

B

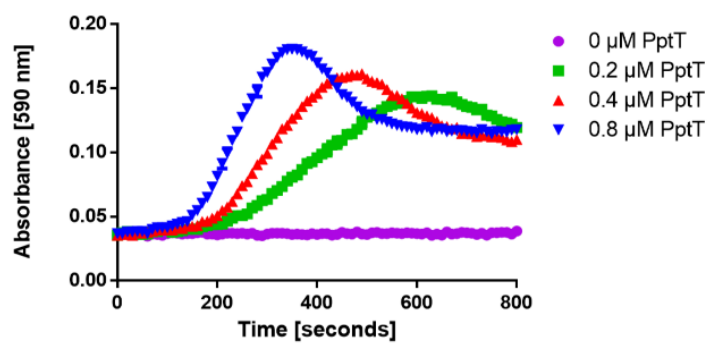

D

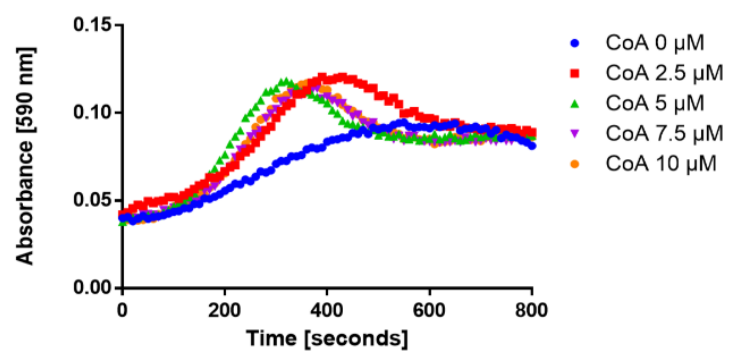

Figure 3.5 Optimisation of reaction parameters: (A) A pH range of $7.8-8.4$ was tested to determine the optimum $\mathrm{pH}$. (B) PptT was tested at a concentration range of $0.2 \mu \mathrm{M}, 0.4 \mu \mathrm{M}$ and $0.8 \mu \mathrm{M}$ to determine the optimal concentration (C) BpsA was tested $0.5 \mu \mathrm{M}, 1 . \mu \mathrm{M} 1.5 \mu \mathrm{M}$ and $2 \mu \mathrm{M}$ to determine the optimal concentration(D) CoA was tested at 2.5 $\mu \mathrm{M}, 5 \mu \mathrm{M} 7.5 \mu \mathrm{M}$ and $10 \mu \mathrm{M}$ to determine the optimal concentration. Data is the average of three technical replicates.

\subsubsection{Optimisation of other parameters}

Both BpsA and PptT require $\mathrm{MgCl}_{2}$ as co-factors, so an excess of $\mathrm{MgCl}_{2}(10 \mathrm{mM})$ was added to each reaction. Excess concentrations of L-GIn and ATP (6 mM) were also added 
to each reaction. A final time of 600 seconds was chosen for the reaction to be monitored. It was reasoned that this would allow the maximum $A_{590}$ values to be determined for positive controls, and screening of six 96 well plates per hour.

\subsubsection{Quantification of PptT inhibition with 6-NOBP}

Since no PptT inhibitors had been reported at the time that this study was initiated in 2012, the broad spectrum PPTase inhibitor 6-NOBP was used to test whether PptT inhibition could be detected. 6-NOBP was first identified as a Sfp inhibitor by Yasgar et al (2010) and it had a reported IC 50 with Sfp of $6.6 \mu \mathrm{M}$. This corresponds to similar values that were experimentally determined for Sfp, PcpS and PP1183 using the BpsA reporter system (Owen et al., 2011).

Work by Owen et al (2011) had also shown that if you increase the CoA concentration, you decrease the sensitivity of the assay. It was therefore unknown not only whether 6NOBP would prove an effective inhibitor of PptT, but also whether the purification of PptT in the presence of CoA might prevent inhibition from being effectively detected.

A two-fold serial dilution from $100 \mu \mathrm{M}$ to $1.6 \mu \mathrm{M}$ of 6-NOBP was set up as described in Section 2.7.2. The $A_{590}$ values were then read every $10 \mathrm{~s}$ and the $I C_{50}$ value was determined by calculating the maximum PPTase velocities as described in methods Section 2.7.4. A four-parameter dose response curve was fitted using the non-linear regression function of Graphpad Prism ${ }^{\odot}$. The calculated $I C_{50}$ of 6-NOBP with $0.4 \mu \mathrm{M}$ of PptT for two biological replicates, each comprising three technical replicates, was 6.8 $\mu \mathrm{M} \pm 1.6(\mathrm{SD})$. This value is similar to the reported $\mathrm{IC}_{50}$ values for other PPTases (between 2-10 $\mu \mathrm{M}$ ) with some variability evident upon changes in enzyme concentration, CoA concentration and/or the method used to detect inhibition. For example, the IC $\mathrm{I}_{50}$ of 6-NOBP with PcpS was $4.0 \mu \mathrm{M}$ with $2.5 \mu \mathrm{M}$ of CoA and it increased to an $\mathrm{IC}_{50}$ of $10.8 \mu \mathrm{M}$ with $10 \mu \mathrm{M}$ of $\mathrm{CoA}$ (Owen et al., 2011). 


\subsection{Development of a high throughput assay to detect the inhibition of PptT}

\subsubsection{Assay design}

Once the PPTase inhibitor assay had been optimised to detect the inhibition of PptT a protocol for HTS in 96 well plates needed to be developed. Several considerations were made in transitioning from a low throughput benchtop assay to a more medium to high throughput assay using liquid handling robots (Acker and Auld, 2014). The chemical library screening concentration was set at $20 \mu \mathrm{M}$. I reasoned that this would allow the detection of inhibitors with a range of activity. The optimisation with 6-NOBP, a strong PPTase inhibitor, had shown that at $20 \mu \mathrm{M}$ complete inhibition occurs.

There was also a cost (of chemical consumption) involved in screening the drug libraries, however at $20 \mu \mathrm{M}$ it was possible to screen the libraries multiple times without consuming a prohibitive amount of drug. All three commercially available libraries are arrayed in 96 well plates in columns 2 -11, with 80 compounds per plate (Fig. 3.7A), with rows 1 and 12 being empty. In addition to screening the compounds on each plate, both a positive (DMSO vehicle only) and a negative control (no PPTase) were assayed. This allowed each assay to be bench marked individually for activity against the vehicle control in case there were small differences between enzyme preparations.

To help maintain compound solubility and to ensure that all of the drug was removed from the pins of the dispensing robot, $28 \mu \mathrm{L}$ of $10 \% \mathrm{v} / \mathrm{v} \mathrm{DMSO}$ in $\mathrm{dd}_{2} \mathrm{O}$ was added to each well in the 96-well screening plate. This, plus the DMSO vehicle in the $2 \mu \mathrm{L}$ of drug solution, resulted in a final concentration of $4.8 \% \mathrm{v} / \mathrm{v}$ DMSO, which I had previously shown has a negligible effect on rate of indigoidine synthesis during my honours thesis. $\mathrm{A} \mathrm{CyBio}^{\circledast}$ liquid handling robot was then used to pin $2 \mu \mathrm{L}$ of drug solution into each well of the plate and $2 \mu \mathrm{L}$ of DMSO into the positive and negative control for a final concentration of $20 \mu \mathrm{M}$. Fifty $\mu \mathrm{L}$ of reaction master mix $(50 \mathrm{mM}$ Tris-Cl pH 8.0, $10 \mathrm{mM}$ $\mathrm{MgCl}_{2}, 6 \mathrm{mM}$ L-GIn, $6 \mathrm{mM}$ ATP, $5 \mu \mathrm{M}$ CoA and $2 \mu \mathrm{M}$ BpsA) was then added to each of the 80 wells containing the arrayed library and the positive and negative controls. The reaction master mix was made fresh for each plate and PptT at a concentration of 0.4 
$\mu \mathrm{M}$ in $20 \mu \mathrm{L}$ of $\mathrm{ddH}_{2} \mathrm{O}$ was then added to initiate the reaction. The plate was shaken at $1000 \mathrm{rev} / \mathrm{min}$ for $10 \mathrm{~s}$ to thoroughly mix the reaction components and then the $A_{590}$ values were recorded every $20 \mathrm{~s}$ for $10 \mathrm{~min}$. Once potential hits had been identified in the library they were then re-screened to eliminate false positives. For example, if there was a pipetting error and PptT was not delivered to a well, the compound corresponding to that well would be identified as a strong inhibitor. After all hits had been validated they were counter screened against holo-BpsA. This ensured that only PptT inhibitors rather than generic BpsA inhibitors or compounds that inhibited other components of the reaction for example $\mathrm{CoA}$ or chelated $\mathrm{MgCl}_{2}$ were identified. The screening workflow is illustrated in Fig. 3.6.

A

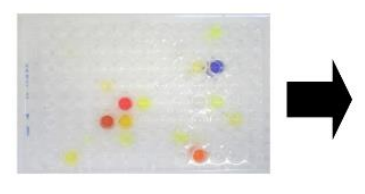

B

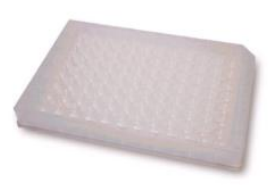

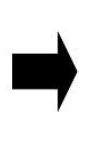

C

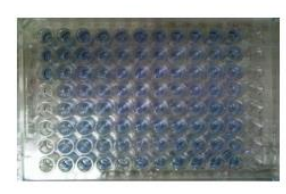

D

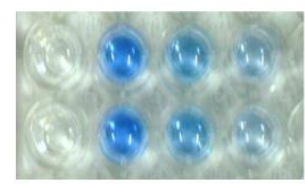

Figure 3.6 Schematic diagram of the HTS workflow: (A) An aliquot from a chemical library is transferred to a 96 well plate (80 compounds per plate) using a $\mathrm{CyBio}^{\circledR}$ robot. (B) A master mix containing the apo-BpsA reaction mix is added to each well, after which the PPTase being screened is added to initiate the reaction. (C) Indigoidine synthesis is monitored over time and 'hits' selected on the basis of diminished indigoidine production as measured spectrophotometrically. (D) Potential hits are validated and then counter screened against holo-BpsA. This ensures the compound is not inhibiting BpsA.

\subsubsection{Kinetic quantification of hits}

To quickly identify potential inhibitors from the large data sets, a classification system was developed to more formally classify inhibitors as weak (less than $25 \%$ inhibition relative to the unchallenged control), medium (greater than $50 \%$ inhibition) or high (greater than75\% inhibition). A mock screening plate was used to determine that the assay could distinguish different levels of inhibition in a 96 well plate format. Three concentrations of 6-NOBP $(20 \mu \mathrm{M}, 10 \mu \mathrm{M}$ or $5 \mu \mathrm{M})$ were seeded into a 96 well screening plate. Each concentration was seeded into two different random wells (Fig 3.7A). The optimised reaction protocol described in Section 3.6.1 was used to screen the plate. The $A_{590}$ values were then recorded and graphed against time (Fig. 3.7C). 6-NOBP at a 
concentration of $20 \mu \mathrm{M}$ (green lines Fig. 3.7C) completely inhibited the synthesis of indigoidine. At a concentration of $10 \mu \mathrm{M}$ (yellow lines Fig. 3.7C) near-complete inhibition was observed, while at a concentration of $5 \mu \mathrm{M}$ (red lines Fig. 3.7C) medium levels of inhibition occurred.

Since some compounds in the chemical libraries interfering with the absorbance measurements of indigoidine, it was decided that rate of indigoidine synthesis would be used to determine the level of inhibition rather than the maximal $A_{590}$ value obtained. Also, some compounds in the library were coloured and could potentially have higher base line $A_{590}$ values (if they were blue for example).

A

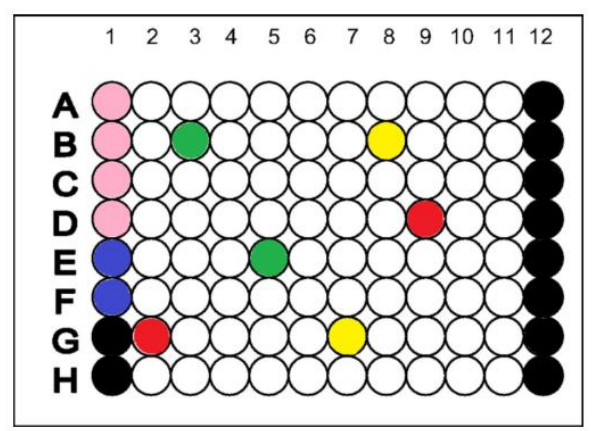

B

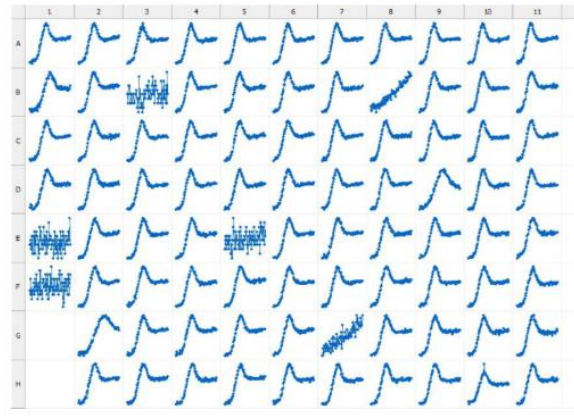

\section{C}

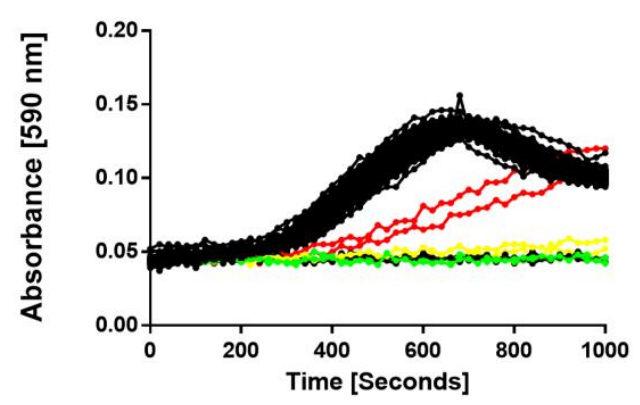

Figure 3.7 Test plate screening: (A) column 1 contains the positive control (pink) and the negative control (blue), empty wells are filled in black. The coloured wells indicate the position of 6-NOBP at different concentrations (red, 5 $\mu \mathrm{M}$, yellow $10 \mu \mathrm{M}$ and green $20 \mu \mathrm{M}$ ). (B) The raw data output from the Perkin Elmer ${ }^{\circledR}$ plate reader, with each well scaled based on its own zero absorbance value. The plate was assayed using the method described in Section 2.7.2. (C) The graphed values from (B). Each concentration of 6-NOBP is highlighted (red, $5 \mu \mathrm{M}$, yellow $10 \mu \mathrm{M}$ and green 20 $\mu \mathrm{M})$. The black lines correspond to the wells in columns $2-11$ and the positive and negative controls in column 1. Wells $\mathrm{G} 1, \mathrm{H} 1$ and column 12 are not assayed. Each line is a single data point. 


\subsection{Identification of PptT inhibitors from the NIH Diversity Set II}

The NIH Diversity set II is a compound library produced by the National Institute of Health (NIH) National Cancer Institute Developmental Therapeutics Programme (DTP). The library contains 1363 compounds arrayed over 17 plates. The NIH Diversity set II is designed to be a broad representation of over 150,000 analogous compounds that are available for ordering from the DTP. The library is arrayed with 80 compounds per plate at a concentration of $10 \mathrm{mM}$ in DMSO. The Diversity Set II was aliquoted into daughter plates that contained a final concentration of $1 \mathrm{mM}$ per well for screening.

\subsubsection{Screening of NIH diversity set II}

The library was screened twice to ensure that if a compound was incorrectly dispensed or the reaction failed to detect inhibition it would be picked up in the repeat of the screen. The library was screened using the optimised protocol described in Section 3.6. Once the library screening had been completed, the raw $A_{590}$ values were graphed and hits were classified based on the reduction in $A_{590}$ values. From the two screens, 14 compounds were validated as potential inhibitors of $\mathrm{PptT}$, with a range of activity at 20 $\mu \mathrm{M}$ from complete inhibition to low levels of inhibition.

The 14 potential inhibitors were then counter-screened against holo-BpsA as described in Section 2.7.3. Counter-screening revealed that nine compounds inhibited the synthesis of indigoidine by holo-BpsA at a concentration of $20 \mu \mathrm{M}$, and these were therefore eliminated from consideration as specific PPTase inhibitors. The structures of the remaining five compounds are displayed in Table 3.1. 


\begin{tabular}{|c|r|r|c|}
\hline Name & Structure & Level of inhibition & Bioactivity $^{1}$ \\
\hline NSC109174 & $<25 \%$ at $20 \mu \mathrm{M}$ & n/a \\
\hline NSC130872 & & $<25 \%$ at $20 \mu \mathrm{M}$ & n/a \\
\hline NSC7962 & & $<50 \%$ inhibition at $20 \mu \mathrm{M}$ & n/a \\
\hline NSC211094 & & $<50 \%$ inhibition at $20 \mu \mathrm{M}$ & Active against Sfp \\
\hline NSC105798 & & & \\
\hline
\end{tabular}

Table 3.2 Top Hits from NIH Diversity set II: Structures are taken from the PubChem compound page and bioassay data is taken from the PubChem Bioassay database. ${ }^{1}$ Data is taken from the QHTS Assay for Inhibitors of Bacillus Subtilis Sfp PPTase bioassay record AID 1490 available from: https://pubchem.ncbi.nlm.nih.gov/bioassay/1490

Of these compounds, only NSC211094 was also identified as an inhibitor of Sfp by the Burkart laboratory, who had looked at approximately 310,000 compounds and identified approximately 11,000 compounds that inhibit B. subtilis Sfp. Their analyses included the compounds arrayed in the NIH Diversity Set II. The screening records from the Burkart study are submitted into the PubChem BioAssay Database under the bioassay record number AID1490, and their data is discussed in Section 1.7. Since four of the five compounds were not identified as inhibitors of Sfp they appear to be specific for PptT. However, all of those compounds had relatively low levels of inhibition, so larger quantities were not purchased in order to determine their $\mathrm{IC}_{50}$ values.

Several of the nine compounds that were identified as potential PptT inhibitors, but were found to inhibit holo-BpsA, were also identified as Sfp inhibitors in the Burkart 
laboratory screen. These included NSC285233 and NSC228155, both of which had detrimental effects on the synthesis of indigoidine. NSC285233 has an IC 50 of $1.2 \mu \mathrm{M}$ and NSC285233 has an IC 50 of $0.5 \mu \mathrm{M}$ against Sfp. This indicates that either they are "frequent fliers", i.e. compounds that exhibit generic protein inhibition, or they are reacting with or otherwise inhibiting a core component present in both screening reactions, for example CoA.

\subsection{LOPAC $^{1280}$}

The LOPAC ${ }^{1280}$ library is described fully in Section 3.2.7 and was kindly provided to this study by the Chemical Genetics Laboratory at Victoria University of Wellington.

\subsubsection{Screening of LOPAC ${ }^{1280}$}

The LOPAC ${ }^{1280}$ library was screened twice in the same manner as described in Section 3.7. From the initial screen, 16 candidate inhibitors were identified. These were then validated and re-screened at a concentration of $20 \mu \mathrm{M}$. From this re-screen, a total of five compounds were identified as potential inhibitors of PptT. Counter screening against holo-BpsA eliminated two strong inhibitors, 4-chloromercuribenzoic acid and 3,4-dichloroisocoumarin. Thus, three inhibitors of PptT were ultimately identified from the LOPAC ${ }^{1280}$ library; ebselen, sanguinarine chloride and suramin hexasodium.

\subsubsection{Validity of the LOPAC ${ }^{1280}$ Library.}

Of concern, several broad spectrum PPTase inhibitors known to be present in the LOPAC $^{1280}$ library were not recovered in this screen. These included 6-NOBP, which has an $\mathrm{IC}_{50}$ of $6.8 \mu \mathrm{M}$ against PptT using the optimised protocol, and should have been easily detected at the screening concentration of $20 \mu \mathrm{M}$. Additionally, several subsequently reported (Vickery et al., 2014) inhibitors of PptT including Bay 11-7085 and SCH-202676, an inhibitor in the nanomolar concentration range, were also not detected.

The LOPAC ${ }^{1280}$ library had previously undergone preliminary screening for inhibitors of PcpS, the essential PPTase from $P$. aeruginosa, and several broad-spectrum inhibitors present in the LOPAC library had successfully been identified. This screening was completed during my honours year, which was several years before the PptT screening 
and I surmised there was the potential that in this time the quality of the library had been degraded or the wells no longer contained enough of certain compounds to be picked up by the CyBio pins.

To determine if this was the case, Bay 11-7085 and SCH-202676, two of the top PcpS inhibitors were tested at a concentration of $20 \mu \mathrm{M}$ against PptT using the optimised protocol described in Section 3.6. As illustrated in Fig. 3.8 both Bay 11-7085 and SCH202676 have complete inhibition at $20 \mu \mathrm{M}$. These results indicate that the LOPAC 1280 library has likely been degraded and hence additional PptT inhibitors could be identified from it if it was in a better condition.

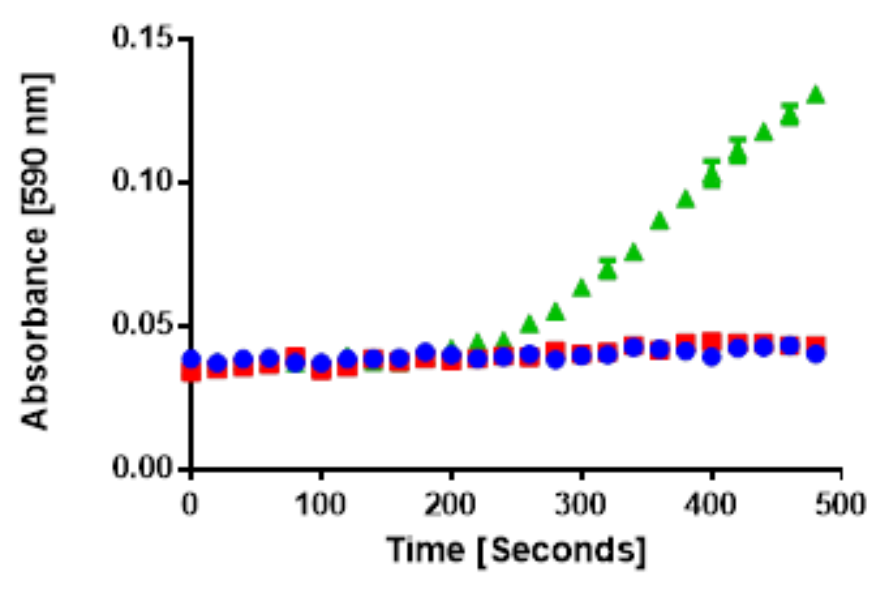

Figure 3.8 Inhibition of PptT by known inhibitors: To determine if the compounds in the LOPAC ${ }^{1280}$ compound library had degraded aliquots of Bay 11-7085 and SCH-202676 that had been obtained more recently were assayed for activity using the optimised protocol described in Section 2.7.2. PptT is completely inhibited by $20 \mu \mathrm{M}$ Bay 11-7085 (red squares) and $20 \mu \mathrm{M} \mathrm{SCH}-202676$ (blue circles) when compared to a DMSO vehicle control (green triangles). This indicates that the LOPAC ${ }^{1280}$ library is degraded. Data is the average of three replicates and error bars represent \pm SEM.

\subsection{Inhibition of PcpS from P. aeruginosa}

\subsubsection{NIH Diversity Set II}

The NIH diversity set II was screened twice for inhibitors of PcpS using the method described in Section 3.7.1. A range of compounds were identified that had inhibitory activity with PcpS. These are shown in Table 3.3. There appears to be more specificity contained within this library - whereas most compounds that inhibit PcpS were found to also inhibit Sfp, several compounds were found that do not inhibit PptT or vice-versa, showing that it is possible to identify compounds that are specific for certain PPTases. In 
total, thirteen compounds were identified as PcpS inhibitors. The structure of the top compounds are displayed in Table 3.3.

\begin{tabular}{|c|c|c|c|}
\hline Name & Structure & Level of inhibition & Bioactivity ${ }^{1}$ \\
\hline NSC43013 & & $\begin{array}{l}>75 \% \\
\text { inhibition at } \\
20 \mu \mathrm{M}\end{array}$ & $\begin{array}{c}\text { Active against } \\
\text { Sfp }\end{array}$ \\
\hline NSC19063 & & $\begin{array}{c}>75 \% \text { inhibition at } \\
20 \mu \mathrm{M}\end{array}$ & $\begin{array}{c}\text { Active against } \\
\text { Sfp }\end{array}$ \\
\hline NSC148170 & & $\begin{array}{c}>75 \% \text { inhibition at } \\
20 \mu \mathrm{M}\end{array}$ & $\begin{array}{c}\text { Active against } \\
\text { Sfp }\end{array}$ \\
\hline NSC98363 & & $\begin{array}{c}>75 \% \text { inhibition at } \\
20 \mu \mathrm{M}\end{array}$ & $\begin{array}{c}\text { Active against } \\
\text { Sfp }\end{array}$ \\
\hline NSC102742 & & $\begin{array}{c}>75 \% \text { inhibition at } \\
20 \mu \mathrm{M}\end{array}$ & $\begin{array}{c}\text { Active against } \\
\text { Sfp }\end{array}$ \\
\hline NSC71033 & & $\begin{array}{c}>75 \% \text { inhibition at } \\
20 \mu \mathrm{M}\end{array}$ & $\begin{array}{c}\text { Active against } \\
\text { Sfp }\end{array}$ \\
\hline
\end{tabular}

Table 3.3: Top PcpS inhibitors identified from NIH Diversity set II: Structures are taken from the PubChem compound page and bioassay data is taken from the PubChem Bioassay database. ${ }^{1}$ Data is taken from the QHTS Assay for Inhibitors of $B$. subtilis Sfp PPTase bioassay record AID 1490 available from: https://pubchem.ncbi.nlm.nih.gov/bioassay/1490

\subsubsection{The Spectrum Collection}

A third commercially available drug library was also screened against PcpS. The Spectrum Collection compiled by MicroSource Discovery contains 2000 compounds, half of which are known drugs. The other half consists of bioactive molecules such as toxins, 
pesticides and herbicides, natural products and pharmacophores having diverse structures but unknown biological activity.

Interestingly, disulfiram was identified in the Spectrum Collection as a strong inhibitor of PcpS, however it was only detected as a weak inhibitor in the LOPAC ${ }^{1280}$ screen, perhaps an early indication of the LOPAC ${ }^{1280}$ quality beginning to degenerate. From the Spectrum Collection, 11 compounds were identified as inhibitors of PcpS. Some of these compounds were not investigated further - for example, merbromin was identified as a strong inhibitor but the known toxicity of the compound ruled it out as a potential drug candidate. Additionally, some compounds that were derived from natural product sources were also removed because of difficulties in acquiring the compound for followup analyses. Several compounds such as lansoprazole, ebselen and disulfiram are present in both the LOPAC ${ }^{1280}$ and the Spectrum Collection. The fact that these compounds were recovered from both libraries as inhibitors of PcpS is a strong validation of the BpsA library screening approach. The final list of novel compounds from the Spectrum Collection found to strongly inhibit PcpS is displayed in Table 3.4.

\begin{tabular}{|c|c|c|c|}
\hline Name & Structure & Level of inhibition & Bioactivity \\
\hline Disulfiram & & $75 \%$ at $20 \mu \mathrm{M}$ & $\begin{array}{c}\text { Active against Sfp } \\
\text { and PptT }\end{array}$ \\
\hline $\begin{array}{c}\text { Lansoprazole } \\
\text { Hydrochloride }\end{array}$ & & $75 \%$ at $20 \mu \mathrm{M}$ & $\begin{array}{c}\text { Active against Sfp } \\
\text { and PptT }\end{array}$ \\
\hline
\end{tabular}

Table 3.4 Top inhibitors identified from the Spectrum Collection: Structures are taken from the PubChem compound page and bioassay data is taken from the PubChem Bioassay database available at https://pubchem.ncbi.nlm.nih.gov/bioassay/1490.

\subsection{Additional libraries}

In addition to the three commercially available libraries, three smaller in-house libraries were also assayed: A New Zealand marine invertebrates extract library consisting of 240 
different extracts provided by Rob Keyzers from the Victoria University School of Chemistry, and two bespoke combinatorial peptide libraries provided by Industrial Research Limited (IRL; now Callaghan Innovation Ltd.), each containing 96 compounds consisting of either tri or di-peptides. One weak hit was identified from the marine invertebrates extract library, however counter screening against holo-BpsA identified it as an inhibitor of either BpsA or another component of the reaction. No PptT or PcpS inhibitors were identified from the two IRL libraries.

\section{$3.12 I_{50}$ values of top PcpS and PptT inhibitors}

The strongest inhibitors identified from either the LOPAC ${ }^{1280}$ or the Spectrum Collection had their $\mathrm{IC}_{50}$ values measured. To calculate the $\mathrm{IC}_{50}$ values, a two-fold serial dilution was used. The maximum PPTase velocity was determined as described in methods Section 2.7.4. This was then used to fit a four-parameter dose-response curve using the nonlinear regression function of GraphPad Prism ${ }^{\circledR}$. The $\mathrm{IC}_{50}$ values for PptT were determined at a PPTase concentration of $0.4 \mu \mathrm{M}$ and the $\mathrm{IC}_{50}$ values of PcpS were determined at 0.2 $\mu \mathrm{M}$ (measuring PcpS at a concentration of $0.4 \mu \mathrm{M}$ resulted in too rapid a reaction, which made it difficult to capture the initial slope). The calculated $\mathrm{IC}_{50}$ values are displayed in table 3.5. Error values represent the standard deviation of three replicates. 


\begin{tabular}{|c|c|c|c|}
\hline Name & Structure & IC 5 PcpS $(\mu \mathrm{M})$ & IC $_{50}$ PptT $(\mu \mathrm{M})$ \\
\hline Bay 11-7085 & & $9.80 \pm 0.2$ & $10.5 \pm 1.0$ \\
\hline PD 404,182 & & $9.3 \pm 0.1$ & $14.8 \pm 0.5$ \\
\hline SCH-202676 & & $0.7 \pm 0.1$ & $2.7 \pm 0.6$ \\
\hline hydrobromide & & $4.3 \pm 0.1$ & $3.6 \pm 0.6$ \\
\hline 6bselen & & $5.4 \pm 0.2$ & $6.8 \pm 1.6$ \\
\hline
\end{tabular}

Table 3.5 IC $\mathrm{C}_{50}$ values of top inhibitors identified across the three libraries: Structures are taken from the PubChem compound page.

\subsection{Anti-microbial activity of SCH-202676 against $P$. aeruginosa}

We were interested in determining if the inhibition of PcpS would translate to a reduction in $P$. aeruginosa viability in vivo. While the effect of inhibiting PptT in $M$. tuberculosis in vivo has previously been examined (Leblanc et al., 2012), similar PPTase inhibition assays have not been performed in P. aeruginosa. Since PcpS activates enzymes involved in both primary and secondary metabolism, and is the sole PPTase in that bacterium, I reasoned that there was potential for it to require less inhibition than the $95 \%$ level of inhibition stated to be required for the PptT homolog to achieve antibacterial effects in M. bovis (Leblanc et al., 2012). An additional advantage of testing PPTase inhibition in $P$. aeruginosa is that it produces an iron chelating siderophore called pyoverdine which is fluorescent and can easily be detected in iron limiting conditions. Pyoverdine is synthesised by the concerted activity of several different NRPSs, each of which need to be post-translationally modified by PcpS before they are functional (Schalk and Guillon, 2013). Thus, I considered that it might be possible to observe a 
reduction in pyoverdine fluorescence at levels of PPTase inhibition that might not prove growth-limiting; or alternatively, if a given compound was antimicrobial for reasons other than PPTase inhibition, pyoverdine production might continue even as bacterial growth rate diminishes.

To determine if the strongest PcpS inhibitor, SCH-202676, had an antimicrobial effect on P. aeruginosa, a serial dilution of $\mathrm{SCH}-202676$ starting at a concentration of $250 \mu \mathrm{M}$ was tested in a growth assay against $P$. aeruginosa in minimal media, as described in Section 2.8. The $\mathrm{OD}_{600}$ values and fluorescent values were measured every $30 \mathrm{~min}$ to monitor both growth and pyoverdine production. As illustrated in Fig. 3.9A, a minor antimicrobial effect was observed when SCH-202676 was tested at a concentration of $250 \mu \mathrm{M}$. Consistent with this, there was a reduction in pyoverdine fluorescence at this drug challenge concentration (albeit not at lower concentrations), suggesting that $\mathrm{SCH}$ 202676 was able to partially inhibit PcpS in vivo (Fig. 3.9B). To ensure that this reduction in fluorescence wasn't due to the reduction in bacterial growth or due to a generic stress response, a serial dilution of the antibiotic gentamycin was also assayed in the same manner. A concentration of gentamycin $(7.8 \mu \mathrm{M})$ was then selected that gave a similar $\mathrm{OD}_{600}$ profile to $\mathrm{SCH}-202676$ and the fluorescence of the culture was monitored at this concentration. At this gentamycin concentration, P. aeruginosa produced similar levels of fluorescence to SCH-202676 at a concentration of $125 \mu \mathrm{M}$, which was not inhibitory to growth (Fig 3.9B). Further work will need to be completed to determine the level of PcpS inhibition required to have an anti-microbial effect. 
A

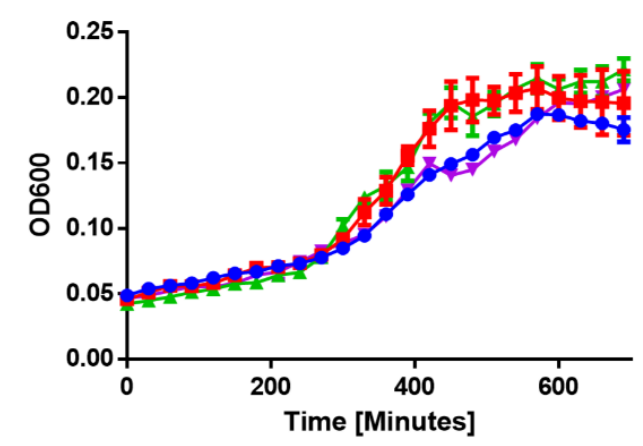

B

Bacterial Fluorescence

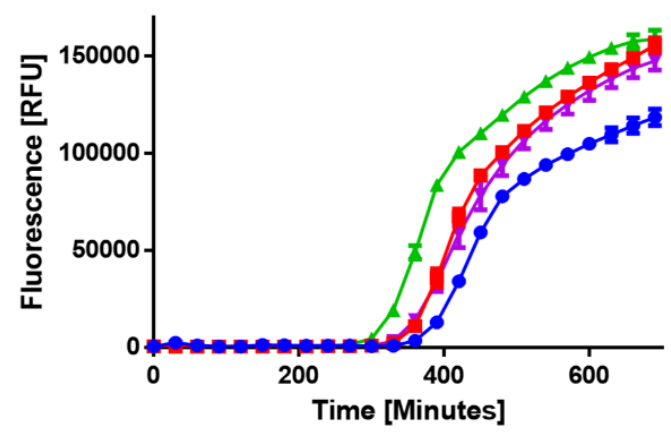

Figure 3.9 Anti-microbial activity of SCH-202676 against $P$. aeruginosa: (A) PA01 was grown in minimal media and the $\mathrm{OD}_{600}$ values were recorded every $30 \mathrm{~min}$. A serial dilution of the antibiotic gentamycin or the PPTase inhibitor SCH-202676 were both assayed. SCH-202676 at a concentration of $250 \mu \mathrm{M}$ (blue circles) and gentamycin at $7.8 \mu \mathrm{M}$ (purple) caused a small reduction in growth compared to the DMSO vehicle control (green triangles). In contrast, $\mathrm{SCH}-$ 202676 at a concentration of $125 \mu \mathrm{M}$ (red squares) didn't cause a reduction in growth. (B) The fluorescent values were measured at $400 \mathrm{em} / 440$ ex for each drug-challenged culture every $30 \mathrm{~min}$. $250 \mu \mathrm{M} \mathrm{SCH}-202676$ (blue circles) caused a reduction in pyoverdine fluorescence when compared to $7.8 \mu \mathrm{M}$ gentamycin (purple triangles), consistent with some PPTase inhibition occurring in vivo. Data is the average of three replicates and error bars are \pm SEM.

\subsection{Single end point screening}

During the development of the glutamine biosensor described in chapter five an assay incorporating a DMSO stop method to solubilise and then measure the total amount of indigoidine synthesised, in an endpoint assay format. I hypothesised it would be possible to apply the same method for PPTase inhibitor screening. Measuring the total amount of indigoidine present in each well might prove advantageous for several reasons: 1) It would reduce the amount of labour required during data processing and the manual handling of reagents; 2 ) It would allow the entire screening process to be automated as liquid handling robots could be used at each step; and 3) Each plate could be screened more rapidly as kinetic measurements over time.

To determine if this method was feasible, a test plate containing 6-NOBP at a concentration of $20 \mu \mathrm{M}, 5 \mu \mathrm{M}$ or $0 \mu \mathrm{M}$ as a negative control was assessed every 15 min for an h. A master mix with the same concentrations as described in Section 3.6 with a volume of $28 \mu \mathrm{L}$ was plated into each plate containing $2 \mu \mathrm{l}$ of 6-NOBP or DMSO. To initiate the reaction, $20 \mu \mathrm{L}$ of PptT at a concentration of $0.4 \mu \mathrm{M}$ in $\mathrm{ddH}_{2} \mathrm{O}$ was added to each well, resulting in a final volume of $50 \mu \mathrm{L}$. The plate was then shaken at 200 $\mathrm{rev} / \mathrm{min}, 30^{\circ} \mathrm{C}$. The reaction was stopped by the addition of $200 \mu \mathrm{L}$ of DMSO every 15 
$\min$. After one $\mathrm{h}$, the entire plate was shaken at $2000 \mathrm{rev} / \mathrm{min}$ for $10 \mathrm{~min}$ to solubilise the indigoidine.

As illustrated in Fig. 3.10 the inhibition of PptT by $20 \mu \mathrm{M}$ 6-NOBP is detectable after an hour. However, the sensitivity of the assay at $5 \mu \mathrm{M}$ of 6-NOBP was much lower.It was concluded that this method would allow the detection of strong PPTase inhibitors but may miss weaker inhibitors. In addition, strongly coloured compounds such as brilliant blue that are present in drug libraries might have to be screened separately to avoid false negatives. This technique was not attempted until the majority of inhibitor screening had been completed, so was not used for further library screening With optimisation it might prove to have utility in future screening efforts.

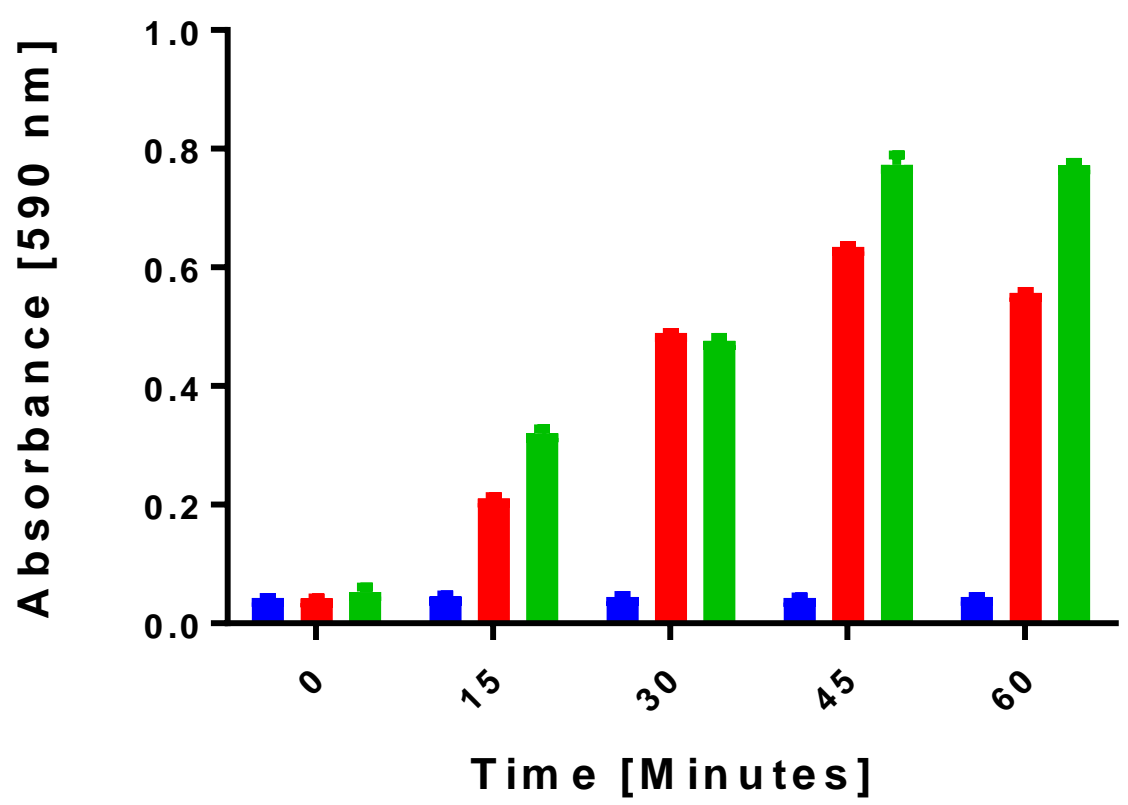

Figure 3.10 Single end point screening: 6-NOBP was assayed at three concentrations; $20 \mu \mathrm{M}$ (blue bars), $5 \mu \mathrm{M}$ (red bars) and $0 \mu \mathrm{M}$ (green bars) using a miniaturised form of the assay described in Section 3.6. The reaction was stopped by the addition $200 \mu \mathrm{L}$ of DMSO and the level of pigment production was quantified. Twenty $\mu$ M 6-NOBP (blue bars) did not show an increase in pigment production indicating complete inhibition of PptT. At a concentration of $10 \mu \mathrm{M} 6$ NOBP (red bars) some indigoidine synthesis was observed. Data is the average of three replicates and the error bars represent \pm SD. 


\subsection{Chapter discussion}

\subsubsection{Development of a screen to detect inhibitors of PptT}

The work in this chapter represents the first high throughput screen for inhibitors of PptT that uses native CoA. Two alternative screens have previously been published, however one requires radiolabelled $\mathrm{CoA}$ and the other requires fluorescent $\mathrm{CoA}$ analogues. Previous work has shown that PptT does not readily accept CoA analogues, potentially due to the unusual " $U$ " confirmation that the P-pant arm forms in the active site, thus the BpsA-based screen is an important step forward in identifying novel PptT inhibitors.

While PptT does not purify easily, by supplementing the buffer that was used to lyse the cells with both $\mathrm{MgCl}_{2}$ and CoA I found the yield and stability of PptT could be increased. It is possible that this purification technique could also be applied to other difficult to purify PPTases, such as AcpS from E. coli or some PPTases from unknown host bacteria that were isolated from environmental samples (Owen et al., 2012). While the CoA and $\mathrm{MgCl}_{2}$ supplementation was found to improve purification, it is possible that this step may have a detrimental effect on the detection of inhibitors, as a lower number of weaker inhibitors were found compared to PcpS (which was purified without any supplementation). A potential future experiment to test this possibility could be to copurify PcpS with CoA and $\mathrm{MgCl}_{2}$ and re-screen a chemical library to determine if this resulted in reduced number of inhibitors being identified. In an attempt to minimise the possible impact of $\mathrm{CoA}$ supplementation, $\mathrm{CoA}$ and $\mathrm{MgCl}_{2}$ were not added to the PPTase storage buffer, a deviation from the original purification protocol described by Rottier et al (2013).

The HTS to detect inhibitors of PptT was optimised to improve signal strength, and to reduce enzyme consumption and the time taken to read each plate. It is possible that further optimisation might still prove useful in enhancing the sensitivity of this screen. For example, in the future it might be beneficial to reduce the concentrations of both BpsA and PptT in the assay and increase the time taken to read each plate. This would reduce the amount of enzyme needed to be purified per plate. Additional parameters could also be further optimised such as temperature and buffer concentrations. 
At a subsequent stage of my PhD research (see Section 3.14) I developed an alternative approach to quantifying BpsA activity, based on an end-point of indigoidine production rather than the initial rate of synthesis. It is possible that this method could be adapted to increase the sensitivity of inhibitor screening, potentially also enabling miniaturisation to a 384 well format. This would consume less drug and enable higher compound concentrations to be tested. It should be possible to miniaturise the assay into a 384 well plate which would reduce the number of reagents required for the assay and increase the number of compounds that could be screened per plate. During the optimisation of the kinetic screen attempts were made to run the assay in a 384 well plate. However, the need to accurately dispense the PPTase in a time-sensitive manner to enable the accurate kinetic measurement of the reaction meant that this was not feasible. The smaller wells also increased the chance of an incorrect dispensing resulting in false positives or false negatives. Future work in this area will focus on the optimisation and further development of this screen.

\subsubsection{Detection of inhibitors of PptT and PcpS}

Several novel inhibitors of PptT were identified from the NIH Diversity Set II. While none of the compounds were strong inhibitors of PptT, there is the potential that analogues of these weak inhibitors could have improved antibiotic properties. Since the Diversity Set II is a representation of a wide range of compounds it should be relatively easy to acquire and identify analogues using the PubChem structure similarity search and related records. In addition to the inhibitors found in the NIH Diversity Set II, other compounds were identified such as SCH-202676 that have high levels of inhibition. Several compounds identified as inhibitors of both PptT and PcpS appear to be broadly active for targets beyond PPTases. For example, Bay 11-7085 is active in 73 out of 392 bioassays deposited in the PubChem bioassay database indicating that it potentially is a frequent flier and may not be worth pursuing further (Baell and Holloway, 2010). Further work will have to be carried out to classify inhibitors as specific PPTase inhibitors and not just broadly inhibitory molecules. Once this has been completed there is the potential to initiate structure activity relationship testing and further medicinal chemistry, as good crystal structures for PptT now exist (Jung et al., 2014) 
Ultimately, none of the PptT inhibitors recovered in this study were tested in vivo. A primary reason for this is that the Ackerley laboratory does not presently have expertise, strains or facilities to safely and effectively culture $M$. tuberculosis. An alternative to this might be to use Mycobacterium smegmatis, a faster growing mycobacterium that is often used as a lab model. To this end the M. smegmatis PptT homolog has been expressed and purified and subjected to preliminary testing in the BpsA screen. Preliminary results (not reported here) have indicated that $M$. smegmatis PptT has slightly less activity than PptT but can still be used to evaluate inhibitors. In the future this may prove a useful model. However, the other main reason for not investing substantial effort into developing mycobacterial models to discern the effects of PPTase inhibition is that the high level of gene knockdown required to get an antimicrobial effect in vivo (Leblanc et al., 2012) coupled to the failure in this study of SCH-202676 to have any proper anti-microbial effect in P. aeruginosa, suggested that substantially improved inhibitors might first need to be generated, either by increasing the number of compounds screened or identifying/ synthesising analogues; both approaches being beyond the scope of this PhD.

\subsubsection{Bioactivity of top inhibitors}

As a possible starting point for future structure activity relationship (SAR) studies, the present work identified a diverse range of small molecule inhibitors as PPTase inhibitors. The top PPTase inhibitors were originally developed for a range of different bioactivities and have a range of different features. These inhibitors are profiled in more detail below.

Bay 11-7085 is a broad spectrum PPTase inhibitor that I found to exhibit IC 50 values of 3.8 $\mu \mathrm{M}, 10.5 \mu \mathrm{M}$, and 9.8 $\mu \mathrm{M}$ against PcpS, PptT and Sfp (Yasgar et al., 2010). Bay 117085 was originally developed as an inhibitor of 1 KB $\alpha$ phosphorylation, preventing activation of NF-KB by cytokines (Pierce et al., 1997). In addition to inhibiting PPTases, Bay 11-7085 has been detected as an inhibitor of a variety of other proteins such as aldehyde dehydrogenase and prostaglandin receptor. According to the PubChem Bioassay Database it is active in 73 of the 392 assays it has been screened in, suggesting that it may be a frequent flier and not likely to have value as a specific and non-toxic antibiotic. 


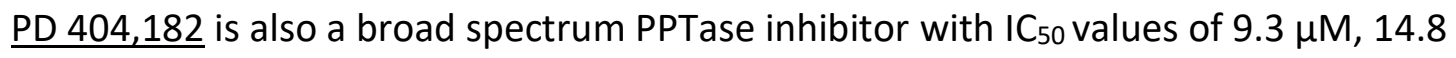
$\mu \mathrm{M}$, and $10 \mu \mathrm{M}$ against PcpS, PptT and Sfp (Yasgar et al., 2010). PD 404,182 was originally developed as a potential inhibitor of the KDO8-P synthase which catalyses the synthesis of KDO, an important part of the lipopolysaccharide layer in Gram-negative bacteria (Mizuhara et al., 2010). Despite being a promising inhibitor, it exhibited little anti-microbial activity in vivo and suffers from poor solubility and a lack of ability to cross the cell wall (Sansom, 2001). However, as the compound targets two different essential proteins then it may yet hold promise attractive as a novel antibiotic, and it may be that it can be engineered to have improved pharmacokinetic profiles.

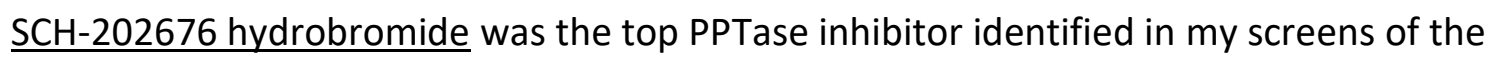
three commercially available drug libraries. It has $I_{50}$ values of; $0.7 \mu \mathrm{M}, 2.7 \mu \mathrm{M}$ and $0.37 \mu \mathrm{M}$ for PcpS, PptT and Sfp. SCH-202676 has been reported as a reversible agonist and antagonist of G-protein coupled receptors (Lewandowicz et al., 2006). Due to the reactive nature of $\mathrm{SCH}-202676$ it was profiled further by Kosa et al (2014). They found that $\mathrm{SCH}-202676$ was relatively fragile and reacted strongly with both reducing agents and other free thiols. In addition to the reactivity SCH-202676 appears to be relatively promiscuous with a hit rate of approximately $10 \%$ in the PubChem database, which would likely reduce its usefulness as a candidate anti-microbial compound (Kosa et al., 2014).

Ebselen was also identified as a PPTase inhibitor with low micromolar IC 50 values (4.3 $\mu \mathrm{M}, 3.6 \mu \mathrm{M}$ and 2.8 $\mu \mathrm{M}$ for PcpS, PptT and Sfp). Despite the low $\mathrm{IC}_{50}$ for Sfp and being present in the LOPAC ${ }^{1280}$ library it was not reported as a top inhibitor by Yasgar et al (2010). Ebselen is an organoselenium compound that has a range of activities. Work by a previous VUW PhD student Dr Chris Miller identified it as a possible anti-tuberculosis compound when screening the LOPAC ${ }^{1280}$ library for activity against $M$. smegmatis. Thus, analogues and other compounds from the same class could provide a rich source of bioactive PPTase analogues.

$\underline{6-N O B P}$ is a well-known PPTase inhibitor with low micromolar IC 50 values $(5.4 \mu \mathrm{M}, 6.8$ $\mu \mathrm{M}$ and 6.0 $\mu \mathrm{M}$ for PcpS, PptT and Sfp) It was originally identified as a compound that binds to the ADP-ribosyltransferase and destabilizes one of the two zinc fingers (Chuang et al., 1993). 6-NOBP has been shown to have anti-fungal activity in vivo (Johns et al., 
2017). It is the only PPTase inhibitor shown to have in vivo activity other than ML267. Thus, 6-NOBP may yet be worth exploring further.

While Bay 11-7085 and SCH-202676 are unlikely to be good candidate drug targets given their off-target effects and generally high reactivity, it is possible that analogues with improved properties could be identified or developed and then screened for activity. Other compounds such as ebselen, PD 404,182 and 6-NOBP could also lead to development of novel antibiotic leads. Despite a substantial number of novel PPTase inhibitors having now been reported in the literature, most have yet to move from the in vitro test stage to anti-microbial testing. Some compounds such as PD 404,182 may also have off target microbial effects that can confound the assays. Additionally, further work should be completed to determine exactly how effective PPTases really are as potential drug targets for example viability studies using gene knockdown. The work described in this chapter suggests that very low levels of PPTase activity are required to support growth, and hence a PPTase inhibitor in the low nanomolar range may be required. This was most evident when evaluating the potential antibacterial activity of $\mathrm{SCH}-202676$, which was tested against the lab strain of $P$. aeruginosa PA01.

While only low levels of anti-microbial activity were detected with SCH-202676 at a concentration of $250 \mu \mathrm{M}$, a decrease in the levels of pyoverdine fluorescence indicated that at least a small amount of PcpS inhibition was occurring within the cell. The reactive nature of $\mathrm{SCH}-202676$ and strong efflux pump systems present in $P$. aeruginosa means that it is likely only a small percentage of the compound was actually inhibiting PcpS. In the future, a panel of PPTase inhibitors could be screened to determine if they have better in vivo properties despite having lower $\mathrm{IC}_{50}$ values in vitro. In addition, a range of $P$. aeruginosa mutants with the efflux pump systems knocked out are available at Victoria University. These mutants would be much more susceptible to PPTase inhibitors. Testing compounds in these strains may improve the in vivo results of certain PcpS inhibitors.

A second option would be to test the inhibitor MLS267 that Foley et al developed to inhibit Sfp. The compound has an IC $\mathrm{C}_{50}$ of $0.290 \mu \mathrm{M}$ against $\mathrm{Sfp}$ and an IC $\mathrm{C}_{50}$ value of greater than $114 \mu \mathrm{M}$ against the human PPTase indicating it is very selective. They also 
showed that it has an in vivo effect (Foley et al., 2014). Measuring the antimicrobial effect of this compound in $P$. aeruginosa should be a priority. 


\section{Chapter 4- Engineering BpsA for improved activity with type I PPTases}

\subsection{Chapter summary}

This chapter focuses on the design and development of an engineered form of BpsA for use in high throughput screening of chemical libraries to identify novel inhibitors of $M$. tuberculosis AcpS (TB-AcpS; a type I PPTase). TB-AcpS has a narrow substrate specificity and does not readily post-translationally modify the PCP of BpsA. Two rationally designed variants were designed, with the second alpha helix from either $M$. tuberculosis AcpM or FAS-I replacing the wildtype helix in the PCP domain of BpsA. Both of these constructs proved to be non-functional. In order to try and recover functionality, a directed evolution campaign using error prone PCR (epPCR) was undertaken to restore activity.

Three small pilot libraries using the wild type BpsA PCP domain (wtBpsA), a rational construct based on AcpM (acpBpsA) and a rational construct based on FAS-1 (facpBpsA) were screened to identify improved variants. Only the library using $\operatorname{acpBpsA}$ as a template showed any detectable pigment production in vivo. The top hits from the pilot screen were carried through two further rounds of evolution. By the third round of evolution, significant improvements in in vivo pigment production was observed. The most improved clones showed detectable pigment production after 8 hours of induction on plates and 24 hours in liquid culture. In comparison, wildtype BpsA had no detectable pigment production in vivo.

However, it became apparent during the isolation and purification process that the endogenous E. coli AcpS (EC-AcpS) present in the host screening strain was activating the improved variants. While the directed evolution campaign may not have improved activity with TB-AcpS it did improve activity with EC-AcpS, however the overall catalytic function of the modified BpsA variants was severely reduced meaning they were not yet at a level of activity to be useful for in vitro assays. Despite the failure to develop a modified BpsA variant capable of detecting the inhibition of TB-AcpS, several key 
residues important for PCP function were identified and targeting these could prove useful should further engineering experiments be undertaken.

\subsection{Introduction}

Like many bacterial species, M. tuberculosis has two functional PPTases, a Type 1 PPTase called AcpS and a type II PPTase called PptT (Cole et al., 1998; Quadri et al., 1998). AcpS is responsible for activating fatty acid synthase I (FAS-1) and at the time this project was initiated was thought to also activate AcpM, the carrier protein subunit from the FAS-II system. However, more recent experimental work has shown this to be incorrect and in fact PptT is responsible for activating AcpM (Zimhony et al., 2015).

Recent papers have focused on developing screens for inhibitors of type II PPTases, with most focusing on the archetypal PPTase Sfp from B. subtilis (Duckworth and Aldrich, 2010; Kosa et al., 2014; Yasgar et al., 2010). Developing a HTS for AcpS type PPTases would be beneficial. PptT inhibitors that can also inhibit TB-AcpS will target an additional essential enzyme to improve anti-microbial activity. Ideally, novel antibiotics will have multiple targets, reducing the potential for antibiotic resistance to develop. One potential source of resistance in M. tuberculosis to PptT inhibitors would be mutations in AcpS that allow it to fulfil the role of PptT, so by targeting both PPTases simultaneously, the possibility of this occurring is lessened. In addition it could result in the identification and discovery of new drug classes that can target type I PPTases but not type II PPTases.

\subsubsection{Structure and Function of AcpS in M. tuberculosis}

The crystal structure of TB-AcpS has similar structural features to most other crystallised type I PPTases (Dym et al., 2009). One significant difference is that the surface of the active sites are moderately electronegative compared to other AcpS type PPTases, which are generally positive. Despite the structural similarity TB-AcpS has low sequence identify when compared to other AcpS type PPTases. TB-AcpS also has an elongated helix followed by a flexible loop as shown in Fig 4.1 (Dym et al., 2009). 

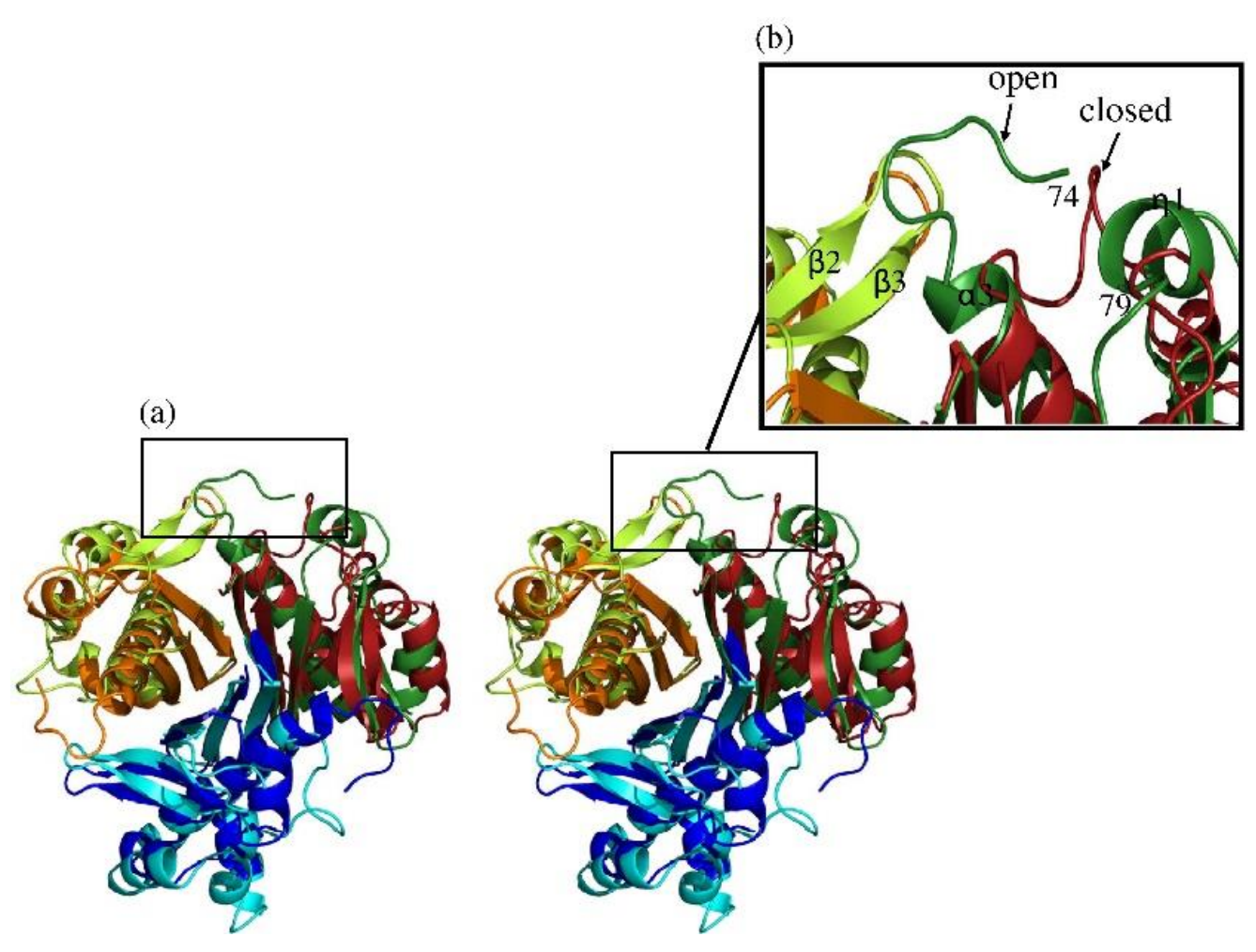

Figure 4.1 Structure of TB-AcpS compared to AcpS from B. subtilis: (A) The TB-AcpS trimer is illustrated in green blue and orange. The $B$. subtilis AcpS trimer is illustrated in red, cyan and lemon. (B) A close up view of the extended loop in the open conformation from AcpS. Reproduced with permission from (Dym et al., 2009)

TB-AcpS was shown to be essential in Mycobacterium species by Chalut et al (2006). A conditional knockout of acpS in M. smegmatis was complemented with a copy of acpS on a heat sensitive plasmid. The $\Delta a c p S$ strain was only viable when the plasmid was present, indicating that AcpS was essential in M. smegmatis. Since AcpS performs the same role in both $M$. smegmatis and $M$. tuberculosis they inferred that it would also be essential in M. tuberculosis. However, this result does conflict with the results from the Sassetti et al transposon library screening of $M$. tuberculosis for essential genes, as acpS was not identified as essential in that screen. Chalut et al acknowledged this by noting that transposon mutant library analysis is not a $100 \%$ percent reliable and can miss some genes (Chalut et al., 2006).

One of the reasons that AcpS was believed to be essential in M. tuberculosis was previous data showing it could activate AcpM in vitro. Gokulan et al showed that AcpS appeared to be able to attach BODIPY-CoA (a modified form of COA that is fluorescent) to AcpM. However, they neglected to run a negative control with BODIPY-CoA and no 
TB-AcpS (Gokulan et al., 2011), which would have shown that it was potentially contaminating EC-AcpS giving a false positive reading.

Subsequent work by Zimhony et al (2015) showed that PptT, not AcpS, activated AcpM. A combination of both SDS-PAGE band shift assays and mass spectrometry analysis was used to identify the correct PPTase that activated AcpM. It is likely that cross contamination from the $E$. coli AcpS in the original Gokulan et al paper was responsible for the activation of AcpM (Zimhony et al., 2015). While it has not been experimentally proven yet it is highly likely that TB-AcpS does activate the FAS-1 protein in $M$. tuberculosis.

\subsubsection{Activation of FAS-1 and AcpM}

One of the hallmarks of $M$. tuberculosis is its extensive and complex lipid-rich cell envelope which helps protect the tubercle bacillus from the host's immune system. The Mycobacterium family cell envelope contains unusual lipids such as mycolic acids, which are the major constituents of this layer. Mycolic acids have other important roles in the lipid membrane, such as providing structural support and protecting the bacterium against oxidative stress (Takayama et al., 2005). As well as mycolic acids, M. tuberculosis also produces a range of other fatty acids such as methyl-branched fatty acids. In order to synthesise these complex lipids, M. tuberculosis contains more than 18 Type-1 polyketide synthetases and two fatty acid synthase systems (Chalut et al., 2006).

The FAS- 1 system is coded by a single gene called fas (Rv2524c). FAS-1 is a homodimer which has the standard domain architecture of a Fatty Acid Synthetase, including the ACP domain. The FAS-1 system provides short-chain fatty acids for acyl-CoA substrates that are further elongated by the FAS-II system. The FAS-II system in contrast consists of several different enzymes including AcpM - the ACP protein, malonyl-CoA:ACP transacylase $(f a b D)$ and $\beta$-ketoacyl synthases (kasA and kasBF) (Gokulan et al., 2011).

\subsubsection{AcpS as a drug target}

While several papers have been published on the potential of Type II PPTases to be drug targets, relatively few papers have been published on Type I PPTases. This is slightly at odds with the general roles of each type of PPTase, as Type I PPTases are involved in 
primary metabolism while Type II PPTases usually have a role in secondary metabolism. For example, entD, the Type II PPTase from E. coli, can be knocked out without any significant effects on growth in rich media (Owen et al., 2012). The small number of papers published on AcpS inhibition have primarily focused on anthranilic acid analogues (Gilbert et al., 2004; Joseph-McCarthy et al., 2005).

One of the first reported AcpS inhibitors was SCH 538415, which was isolated from an unidentified bacterium (Chu et al., 2003). Chu et al used the attachment of radiolabelled 4' phosphopantetheine to preparations of ACP to measure the level inhibition of AcpS. A HTS program for inhibitors identified SCH 538415 from a bacterial extract. This compound was then isolated and the structure was solved. SCH 538415 had an $\mathrm{IC}_{50}$ of 4.19 $\mu \mathrm{M}$ against AcpS in vitro and had detectable anti-microbial activity against S. aureus using a zone of inhibition assay (Chu et al., 2003).

Gilbert et al (2004) published a paper on structural based design of novel anthranilic acid AcpS inhibitors. A FRET based HTS for inhibitors of $B$. subtilis AcpS identified a number of anthranilic acids as lead compounds, the best of which had an $\mathrm{IC}_{50}$ of $15 \mu \mathrm{M}$ against AcpS. However, this compound had no MIC activity against a range of different bacterial strains (Gilbert et al., 2004). Analysis of the compound identified a lack of membrane permeability as being the likely issue. A range of anthranilic acid analogues were then synthesised with the aim of overcoming this limitation, and one of these was shown to have an $\mathrm{IC}_{50}$ for $0.27 \mu \mathrm{M}$ against AcpS and an MIC of $25 \mu \mathrm{M}$ against $B$. subtilis (Gilbert et al., 2004).

A follow up paper by Joseph-McCarthy et al (2005) further developed some of the analogues identified in the Gilbert et al (2004) paper. A different anthranilic acid was identified as the lead compound for this study, which had a relatively weak $I C_{50}$ of 32.3 $\mu \mathrm{M}$ against $B$. subtilis AcpS, but was perceived to have greater potential for optimisation (Joseph-McCarthy et al., 2005). The authors sought to improve both the $I_{50}$ values of the inhibitor and improve its undesirable properties using structure based design. From a screen of analogues, one was identified that had significantly improved potency (1.4 $\mu \mathrm{M} I \mathrm{C}_{50}$ against $\mathrm{AcpS}$ ) and did not damage the membrane of $B$. subtilis (Joseph-McCarthy et al., 2005). Further work has yet to be published on this line of inhibitors and it appears this area of research may no-longer be of interest, perhaps due to the poor in 
vitro antibacterial activity. Of concern, this work did not appear to be repeatable; Foley et al synthesised the lead compound to use as a benchmark for the development of ML267, a top Sfp inhibitor (Foley et al., 2014), and when it was assayed for inhibitory activity against both $\operatorname{Sfp}$ and AcpS from $B$. subtilis it was found to have no activity with either (Foley et al., 2010).

Due to the small number of chemical screens conducted for Type I PPTase inhibitors there are few lead compounds and the general classes of inhibitors that are active against Type I PPTases are unknown. By employing BpsA to screen for TB-AcpS inhibitors I sought to identify new classes of inhibitors. I hoped to also be able to identify PptT inhibitors that additionally exhibited activity with TB-AcpS, which might improve their bactericidal effect and reduce the chance of $M$. tuberculosis developing resistance via mutations in TB-AcpS.

\subsubsection{Engineering PCP domains for improved activity with type I PPTases}

It was unlikely that TB-AcpS would have any activity with BpsA. Type I PPTases typically have little to no activity with PCP domains. BpsA is also routinely purified from $E$. coli $\Delta e n t D$ that contains the essential PPTase EC-AcpS and it is purified almost entirely in the inactive apo form indicating that the PCP domain is not a substrate for AcpS (Owen et al., 2011).

Several papers have probed the interactions between carrier proteins and incompatible PPTases and have introduced modifications to improve the recognition. Research by Mofid et al (2002) showed that it is possible to improve the recognition between Type I PPTases and PCP domains (Mofid et al., 2002). In their landmark paper, they took the second alpha helix from an ACP in B. subtilis which is recognised by AcpS and then replaced the corresponding second alpha helix from the PCP domain of TycC3, an NRPS involved in the synthesis of tyrocidine. The chimeric PCP domain was able to be posttranslationally modified by AcpS, albeit with a $\mathrm{K}_{M}$ three times lower than the native ACP substrate. Despite this slowing of catalytic efficiency, it still had an increased level of overall functionality (Mofid et al., 2002).

Further work by Finking et al (2004) narrowed down the residues involved in substrate selection within the second alpha helix of the PCP domain of TycA (Finking et al., 2004). 
By modifying a single residue within in the helix to a positively charged amino acid such as arginine or lysine which is common in ACP domains, the PCP domain was accepted as a substrate by AcpS. The catalytic efficiencies of these single residue variants were significantly lower compared to native ACP (Finking et al., 2004). Despite the reduction in catalytic efficacy, the fact that single mutation could result in the PCP being post translationally modified by AcpS indicated that the two types of carrier protein were potentially compatible and that it should be possible to develop a modified version of the PCP domain of BpsA that can be modified by TB-AcpS.

\subsubsection{Engineering the PCP domain of BpsA}

Previous research has also shown that it is possible to modify the PCP domain of BpsA to be recognised by a greater range of PPTases. This was first reported by Owen et al (2012) who exchanged the PCP domain of BpsA with other CP domains from E. coli and $P$ aeruginosa and then used directed evolution to improve activity. Error prone PCR identified two key residues at the +4 and +24 positions in respect to the conserved serine residue for functionality (Owen et al., 2016). Results from the directed evolution experiment and modelling by Frueh et al of the structure of the PCP-TE domain of EntF indicated that at the +4 and the +24 position hydrophobic residues were important for the mobility of the PCP domain and its interaction with the TE domain (Owen et al., 2016). This led to the development of novel reporters for PPTase activity in metagenomic clusters (Owen et al., 2016), work that is reviewed in more detail in Section 1.11.3.

Beer et al (2014) also substituted alternative PCP domains into an indigoidinesynthesising NRPS. They used the BpsA homolog IndC from Photorhabdus luminescens, and developed a range of synthetic PCP domains that increased the in vivo synthesis rate of indigoidine. Three different algorithms were used to guide the development of novel PCP domains, including identification of the consensus sequence from multiple sequence alignments, guided random sampling of residues, and random generation. From these outputs, seven synthetic PCP domains were built. Of these, the PCP domain SynT1, built based on data from the consensus algorithm, had improved rates of indigoidine synthesis (Beer et al., 2014). The paper doesn't identify exactly why this is the case, i.e. whether it improves the overall catalytic efficiency of the recombinant 
BpsA or the activity between Sfp (the PPTase used to convert BpsA to holo form) and BpsA. Since no in vitro kinetics were measured, it is difficult to confirm which facet of PCP domain function had been improved. An interesting aspect of the paper was their discovery that the linker region between the PCP domain and the A domain and the linker region between the PCP domain and TE domain plays a substantial role in the level of activity of PCP domain swaps. Beer et al transformed in the PCP domain from BpsA using boundaries that had been delineated using the Pfam tool. Surprisingly, the modified IndC construct with the BpsA PCP domain had no activity despite the two PCP domains being derived from very close homologs. By increasing the size of the boundaries determining the edge of the incoming BpsA PCP domain, Beer et al were able to build a functional IndC construct (Beer et al., 2014). This indicated that the predicted linker regions between the PCP domain, A domain and TE domain are important. This observation regarding the importance of boundary regions was of interest for the current work, however because I aimed to only modify the second alpha helix of the PCP domain I considered that a similar scenario would be unlikely to arise.

\subsection{Research Aims}

- To modify BpsA using both rational engineering and directed evolution to have improved activity with TB-AcpS.

- If successful in Aim 1, to use the top improved mutant to screen for TB-AcpS inhibitors. 


\subsection{Determination of TB-AcpS activity with BpsA}

While it was viewed as highly unlikely that TB-AcpS would have activity with BpsA, this had yet to be confirmed experimentally.

\subsubsection{Cloning, expression and purification of AcpS}

To attach an $\mathrm{N}$-terminus $6 \mathrm{His}$ tag, the acpS gene was cloned into the $\mathrm{pET}-28 \mathrm{a}(+)$ plasmid using Ndel and Sall restriction sites from M. tuberculosis H37Ra genomic DNA. The construct was then sequence verified and transferred to E. coli Bl21 DE3 for protein and expression and purification. TB-AcpS was expressed and purified using the standard protocol described in Section 2.5.5.2 with minor modifications as described in the following paragraph.

TB-AcpS is more soluble when expressed in the BL21 expression system than Type II PPTases such as PcpS and PptT. Despite the increased solubility, it doesn't readily bind to the Ni-NTA column, which potentially could be due to the trimeric structure of TB-AcpS. In order to achieve the high yields needed for screening, a modified low imidazole purification method was used with lower levels of imidazole in the bind buffer and wash buffer (see Section 2.5.5.2). Since TB-AcpS is more stable compared to other PPTases, an Amicon Ultra-15 ${ }^{\circledR} 10 \mathrm{kDa}$ cut off column was then used to desalt the protein and exchange the elution buffer with PPTase desalting buffer. This modified purification protocol resulted in substantial yields of soluble TB-AcpS (typically three times the yield from a $400 \mathrm{ml}$ culture compared to Type II PPTases), as illustrated in Fig. 4.2. The protein was then snap frozen in aliquots at $-80^{\circ} \mathrm{C}$ for use in in vitro kinetic assays. 


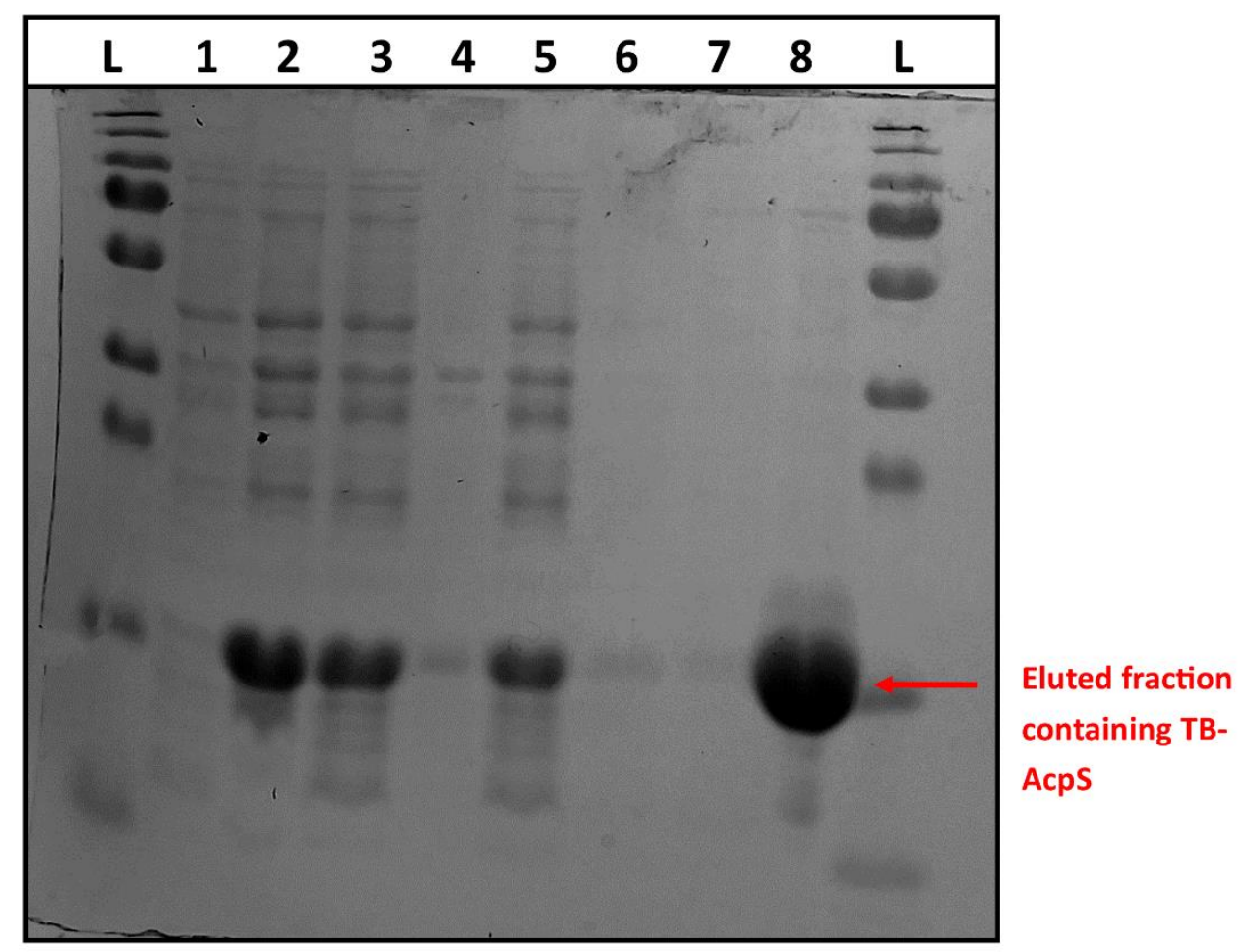

Figure 4.2 SDS-Page gel showing the TB-AcpS purification protocol: TB-AcpS was purified as described in Section 2.5.5.2. The TB-AcpS monomer has an approximate molecular weight of $14 \mathrm{kDa}$ and the eluted, purified TB-AcpS is indicated by the red arrow. The lanes in the SDS page gel correspond to the following samples: L) Molecular weight ladder 1) Pre-induction sample 2) Twenty four hour post-induction sample 3) Soluble fraction following lysis 4) Insoluble fraction following lysis 5) Elution collected after sample applied to the column 6) Elution collected after bind buffer was applied to the column 7) ) Elution collected after wash buffer was applied to the column 8) ) Elution collected after elution buffer was applied to the column. Twelve $\mu \mathrm{L}$ of sample, resuspended in $3 X$ SDS loading buffer was added to each lane.

\subsubsection{Activity with BpsA in vivo}

A key reason that it was considered unlikely that AcpS would be able to attach a Ppant moiety to the PCP domain of BpsA is that Type I PPTases characteristically exhibit narrow substrate specificity. Previous work by Owen et al (2012) has shown that BpsA can be activated by a range of different PPTases, but none of the novel PPTases identified from gDNA were Type I PPTases (Owen et al., 2012). Furthermore, EC-AcpS is also incapable of activating BpsA. To test the in vivo ability of TB-AcpS to activate BpsA and produce detectable pigmentation, a strain of BL21 that had the native type II PPTase entD knocked out, and is hence incapable of converting BpsA to the active holo form, was used. BI21 $\Delta e n t D$ harbouring pET28a(+)::acpS and pCDFDUET::bpsA was plated on pigment producing agar (Section 2.9.6). Following 16 hours of incubation at $37^{\circ} \mathrm{C}$, 
expression of both TB-AcpS and BpsA was induced using the lac expression system with $120 \mu \mathrm{L}$ of 3\% IPTG being spread underneath the agar and allowed to diffuse up through the agar. This method of induction was required as indigoidine is mildly anti-microbial and expressing indigoidine immediately would be detrimental to growth. After $72 \mathrm{~h}$ at $25^{\circ} \mathrm{C}$, no colonies had produced any detectable levels of pigmentation. In contrast, when BpsA was co-expressed with PptT, strong pigmentation was observed four hours after induction (Fig 4.3A).

\subsubsection{In vitro activity with BpsA}

Previous work with PPTases and PCP domains that exhibited low levels of activity has shown that monitoring indigoidine synthesis in vitro is a more sensitive method than whole cell in vivo assays (Owen, 2010). To determine if TB-AcpS exhibited any detectable in vitro activity with BpsA, a kinetic assay was set up as described in Section 2.7.1 with the following modifications: Purified TB-AcpS at a concentration of $1 \mu \mathrm{M}$ and $5 \mu \mathrm{M}$ in 20 $\mu \mathrm{L}$ of $\mathrm{ddH}_{2} \mathrm{O}$ was added to a reaction master mix to initiate the reaction, and $\mathrm{A}_{590}$ values were recorded every $20 \mathrm{~s}$ for an h. A positive control of $1 \mu \mathrm{M}$ PptT and a negative control of no PPTase was also assayed.

AcpS at concentrations of 1 and $5 \mu \mathrm{M}$ was only able to activate BpsA at levels just above the base line levels of activity (Fig 4.3B). I considered that the level of BpsA activation and the amount of indigoidine produced would be too low to reliably detect the conversion of apo-BpsA to holo-BpsA during inhibitor screening. Additionally, increasing the AcpS concentration was not feasible as one of the main bottlenecks in the inhibitor screening process is the expression and purification of enzyme. Increasing the concentration of AcpS would also result in needing higher concentrations of inhibitors. 
A

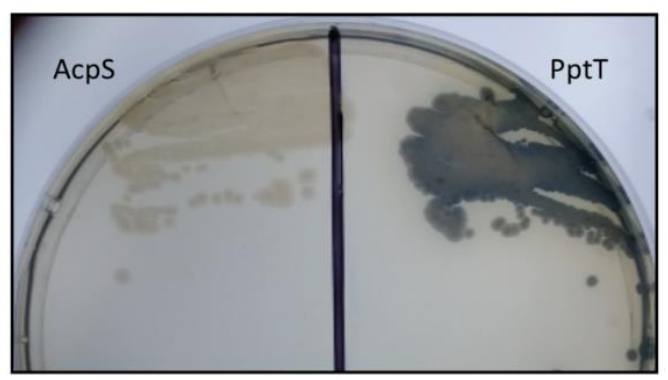

B

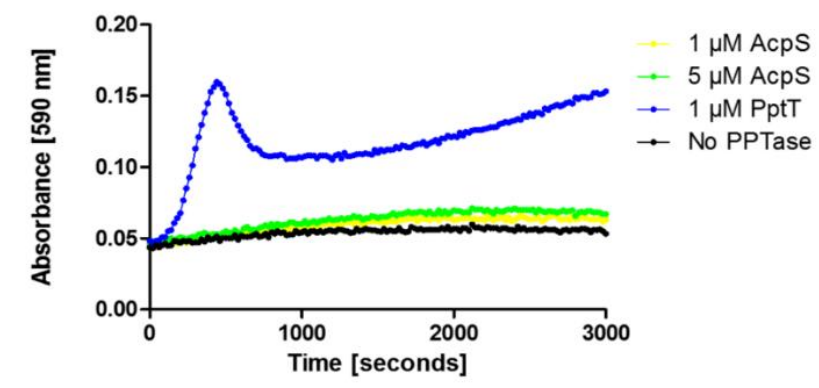

Figure 4.3: Activation of BpsA by AcpS both in vivo and in vitro: (A) BpsA and PptT or AcpS were co-expressed on plasmids with IPTG inducible expression in an $E$. coli $\triangle$ entD strain incapable of post-translationally modifying BpsA unless a PPTase is co-expressed Both strains were streaked on pigment producing media described in Section 2.9.6. The photo was taken six hours after induction with $120 \mu \mathrm{L}$ of 3\% IPTG using the bottom spreading method developed by Owen (Owen, 2010). This photo illustrates the difference in activation between AcpS and PptT. (B) The average $A_{590}$ values of three replicates are graphed every 20 seconds. PptT exhibits the characteristic curve of holo BpsA producing indigoidine in contrast AcpS poorly activates BpsA even at higher concentrations.

\subsection{Rational design of improved BpsA variants}

Two rationally designed constructs were developed using native carrier protein substrates of TB-AcpS. The first of these utilised the second alpha helix from AcpM, which at the time of construction had been indicated to be a substrate of TB-AcpS (Gokulan et al., 2011). The second construct employed the second alpha helix of the $M$. tuberculosis FAS-1 construct. The second alpha helixes were substituted rather than the entire CP domain as work by Mofid et al and Finking et al (Finking et al., 2004; Mofid et al., 2002) had shown that substituting the second alpha helix could improve the activity between AcpS and the PCP domain of an NRPS. I reasoned that by limiting the number of residue changes in the PCP domain to the second alpha helix. I could more easily restore activity to the BpsA PCP domain using directed evolution if the constructs were non-functional.

\subsubsection{Design of the acpBpsA construct}

The BpsA PCP second alpha helix was substituted by the second helix of AcpM from $M$. tuberculosis and the resulting construct was called acpBpsA. At the time that this research was initiated, the work by Zimhony et al in 2015 had not yet been published 
and the prevailing evidence was that TB-AcpS activated AcpM (Zimhony et al., 2015). The structure of AсpM has been solved and alignments show that it exhibits high sequence similarities with ACPs from other bacterial species (Wong et al., 2002). When aligned with the second alpha helix from BpsA it has a low level of sequence similarity, with only three residues being conserved, one of which is the serine conserved in all carrier proteins (Fig 4.4).
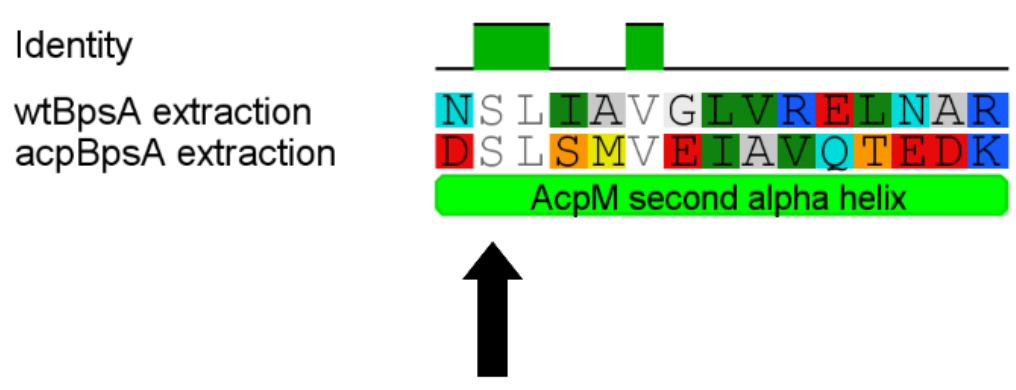

Figure 4.4 Alignment of the second alpha helix of AcpM with the second alpha helix of BpsA: The black arrow indicates the conserved serine to which the Ppant arm is attached.

\subsubsection{Construction of acpBpsA}

A vector for the seamless integration of non-native PCP domains into BpsA was developed by Dr Jeremy Owen as part of his PhD research. This vector called pSLBpsA consisted of BpsA cloned into the PCDFDUET vector, with silent restriction sites (the nucleotide sequence is modified without changing the amino acid sequence) in the encoded loop regions either side of the PCP domain. This allows the seamless integration of PCP domains (Owen, 2010) into BpsA. A gblock ${ }^{\circledR}$ from IDT was synthesised to enable insertion of the second alpha helix from AсрM in place of the native BpsA helix. PCR with SL_BpsA_FWD and SL_BpsA_REV primers was then used to amplify the modified PCP domain, which was cloned into the seamless swapping vector (Section 2.9). The construct was then sequenced to ensure no mutations had been introduced in the process and it was inserted correctly in frame.

\subsubsection{In vivo and in vitro testing of acpBpsA}

The acpBpsA construct was expressed and purified in BL21 $\Delta$ entD cells using the standard BpsA expression and purification methods described in Section 2.5. The eluted fraction displayed the characteristic yellow colour of FMN-containing protein, indicating 
that the oxidation domain was intact and the construct was soluble, the latter aspect being confirmed by SDS-PAGE analysis. AcpBpsA had similar yields and solubility to wtBpsA, indicating that the modification of the second alpha helix did not have a detrimental affect on the stability of the enzyme.

AcpBpsA was co-expressed with both TB-AcpS and PptT in BL21 $\Delta e n t D$ and colonies were plated on pigment producing media in the same manner as described in Section 2.9.6. After $72 \mathrm{~h}$ post induction with IPTG at $25^{\circ} \mathrm{C}$, there was no noticable pigment produced when co-expressed with both $M$. tuberculosis PPTases. This indicated that acpBpsA had lost functionality with PptT and hadn't gained function with TB-AcpS in vivo. To determine the extent of the loss of function, in vitro characterisation was undertaken.

AcpBpsA was tested against both TB-AcpS and PptT at a concentration of $1 \mu \mathrm{M}, 5 \mu \mathrm{M}$ and $0.5 \mu \mathrm{M}$ respectively (as described in Section 4.3.3). AcpBpsA had no activity with either PPTase (Fig 4.6). This indicated that the functionality of the modififed PCP domain had been severely reduced by the replacement of the second alpha helix

\subsubsection{Design and construction of the facpBpsA construct}

The second rationally designed construct was developed based on the second alpha helix from the ACP domain of M. tuberculosis FAS-1 and called facpBpsA. Fernandes et al had previously completed a characterisation of the $M$. bovis FAS-1 protein, which included identifying the conserved serine that the phosphopantetheine arm is attached to (Fernandes and Kolattukudy, 1996). The FAS-1 amino acid sequence from M. bovis was aligned against the FAS-1 amino acid sequence from $M$. tuberculosis in order to identify the second alpha helix. The 12 amino acids surrounding the conserved serine were identified and inserted into the PCP domain of BpsA (Fig. 4.5). Work with other PPTase tags including the S6 and A12 tags (as described in Section 1.8) had shown that 12 amino acids was enough to act as an effective substrate for their respective PPTases (Zhou et al., 2007). Since the acpBpsA construct also had reduced functionality with PptT, it was hoped that by shortening the tag to the minimum amount necessary to promote binding it might not reduce the catalytic efficiency of the PCP domain of the facpBpsA construct to the same extent as was evident in acpBpsA. 
The construct was designed and cloned into the seamless swapping vector in the same manner as described in Section 4.4.2. FacpBpsA was expressed and purified using the protocol described in Section 2.5. SDS-PAGE analysis revealed that the construct was soluble, and had similar solubility and yield characteristics to wtBpsA and acpBpsA, indicating that modifying the second alpha helix of the PCP domain had not had a detrimental effect on solubility.
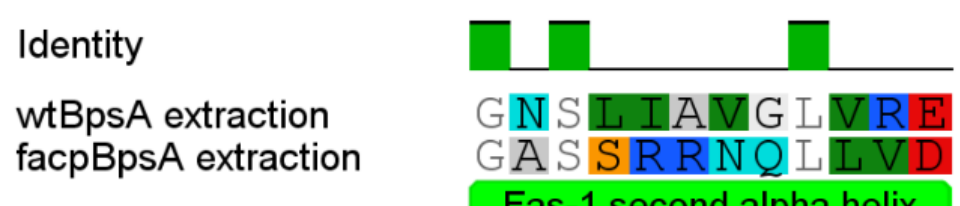

Figure 4.5 Alignment of facpBpsA with wtBpsA: The black arrow indicates the conserved serine to which the Ppant arm is attached.

\subsubsection{In vivo and in vitro testing of facpBpsA}

FacpBpsA was tested in vivo in the same manner as acpBpsA, as described in Section 4.4.3. No pigment production was detected when facpBpsA was co-expressed in vivo with either PptT or TB-AcpS. In vitro testing with both PptT at $1 \mu \mathrm{M}$ and TB-AcpS at 1 and $5 \mu \mathrm{M}$ resulted in no detectable pigment production with either PPTase, consistent with facpBpsA having lost all activity (Fig 4.6). 


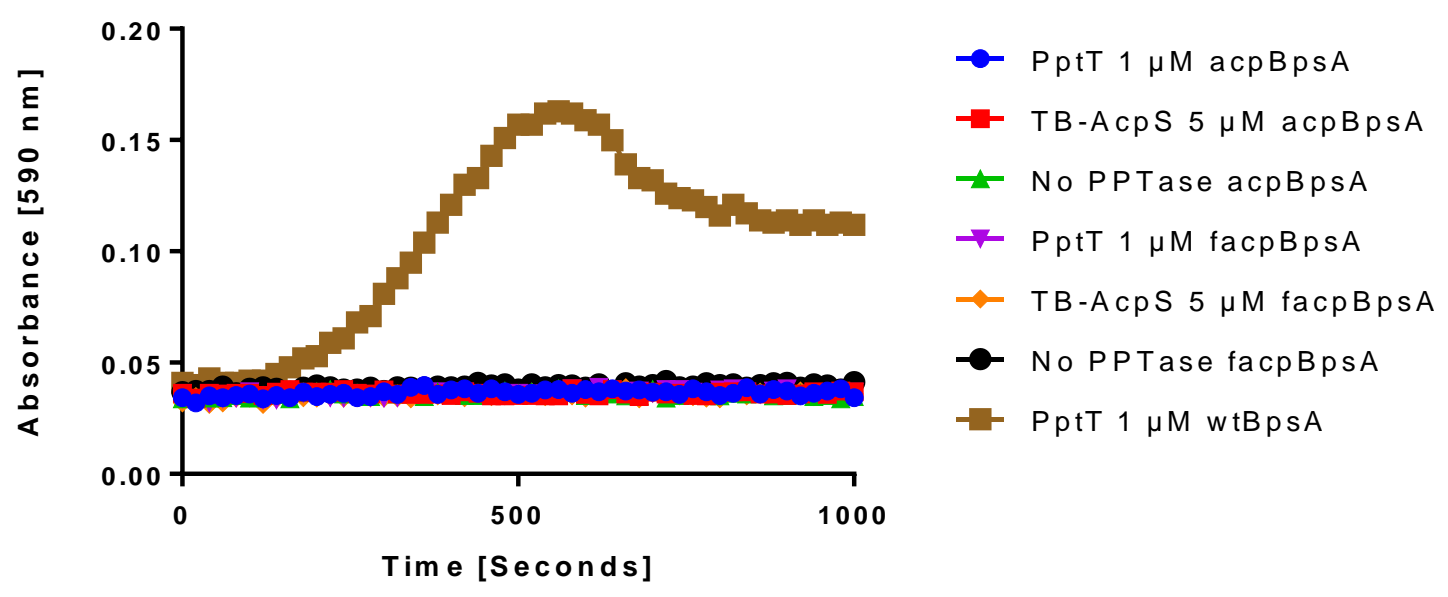

Figure 4.6 In vitro activity of acpBpsA and facpBpsA with TB-AcpS and PptT: acpBpsA and facpBpsA at a concentration of $3 \mu \mathrm{M}$ were assayed for activity with PptT at a concentration of $1 \mu \mathrm{M}$ and TB-AcpS at a concentration of $5 \mu \mathrm{M}$. AcpBpsA and facpBpsA did not synthesise any detectable indigoidine with either PPTase indicating they were not converted into their active holo form. The positive control of wtBpsA with PptT at a concentration of $1 \mu \mathrm{M}$ synthesised indigoidine normally. Data is the average of three technical replicates.

\subsection{Development of pilot libraries for directed evolution}

Previous work by Owen et al $(2012,2016)$ showed that directed evolution could be used to improve the activity of non-native PCP domains within BpsA (Owen et al., 2016). In an attempt to improve the recognition between TB-AcpS and either wtBpsA, acpBpsA or facpBpsA, a directed evolution campaign was undertaken (Fig. 4.7) Three small pilot libraries, using each construct as a template, were constructed and screened against TBAcpS. The reasons for developing pilot libraries first was twofold: Firstly, I considered that it would provide some insight into the "mutability" of each construct. Previous studies, both for the second alpha helix and PCP domain as a whole, had shown that small changes in the amino acid sequence can result in a restoration of function (Mofid et al., 2002; Owen et al., 2016). It was hoped that one or two residue substitutions might be sufficient to restore functionality here. Secondly, by using several templates instead of the single sequence from each construct for the main evolution campaign I aimed to avoid traveling up evolutionary cul-de-sacs. These cul-de-sacs can happen when you reach a local peak of improvement on the fitness landscape but then cannot improve the mutant any further during subsequent rounds of evolution. Previous directed evolution campaigns in the laboratory had shown that amplifying multiple templates introduces a "shuffling" effect which could help us remove deleterious mutations and improve the overall rate of evolution. This is especially important for this evolution campaign as I was 
using a screen rather than a selection, which limits the size of the library that can effectively be interrogated. Fig. 4.7 illustrates the directed evolution process.
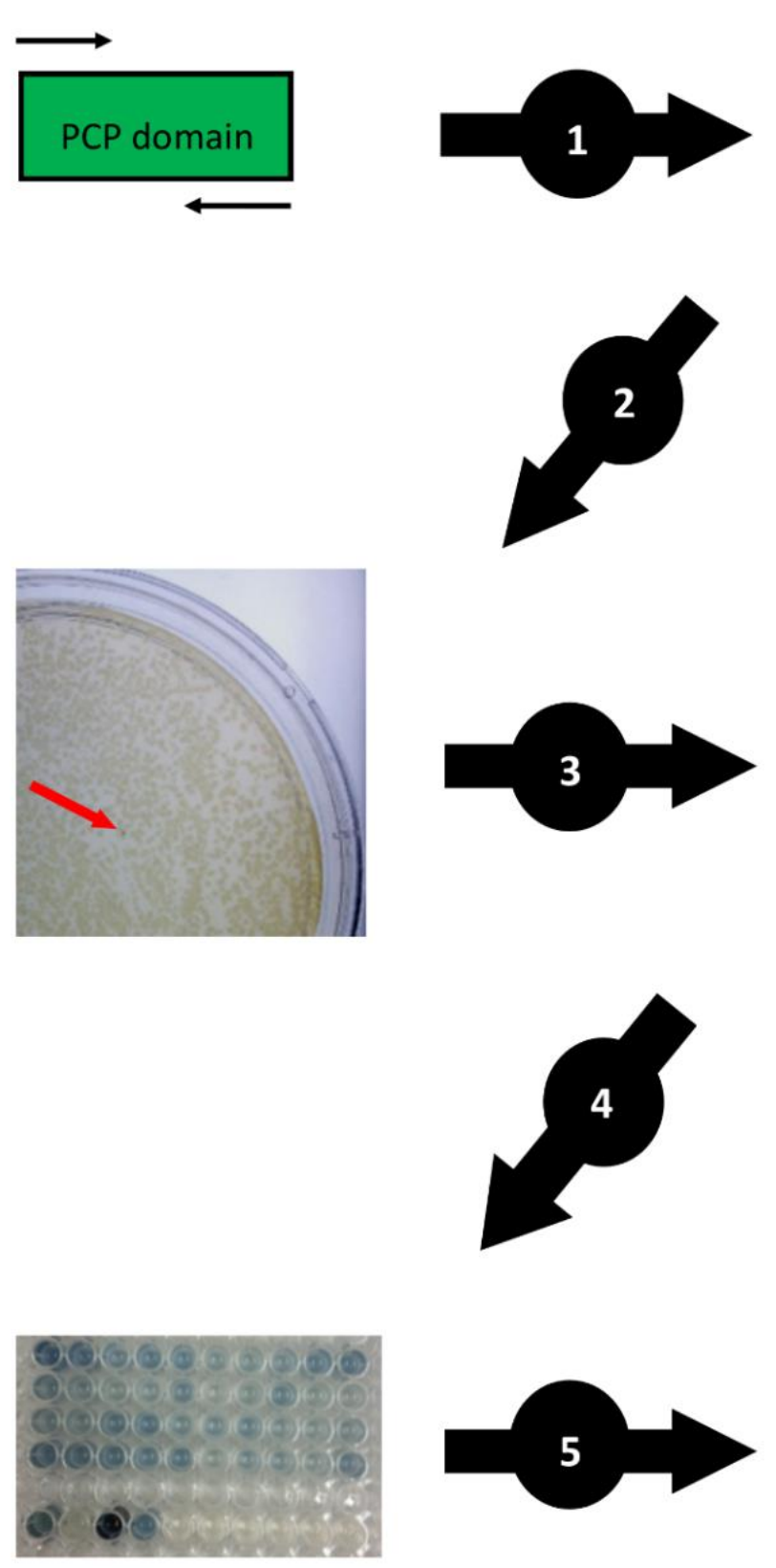
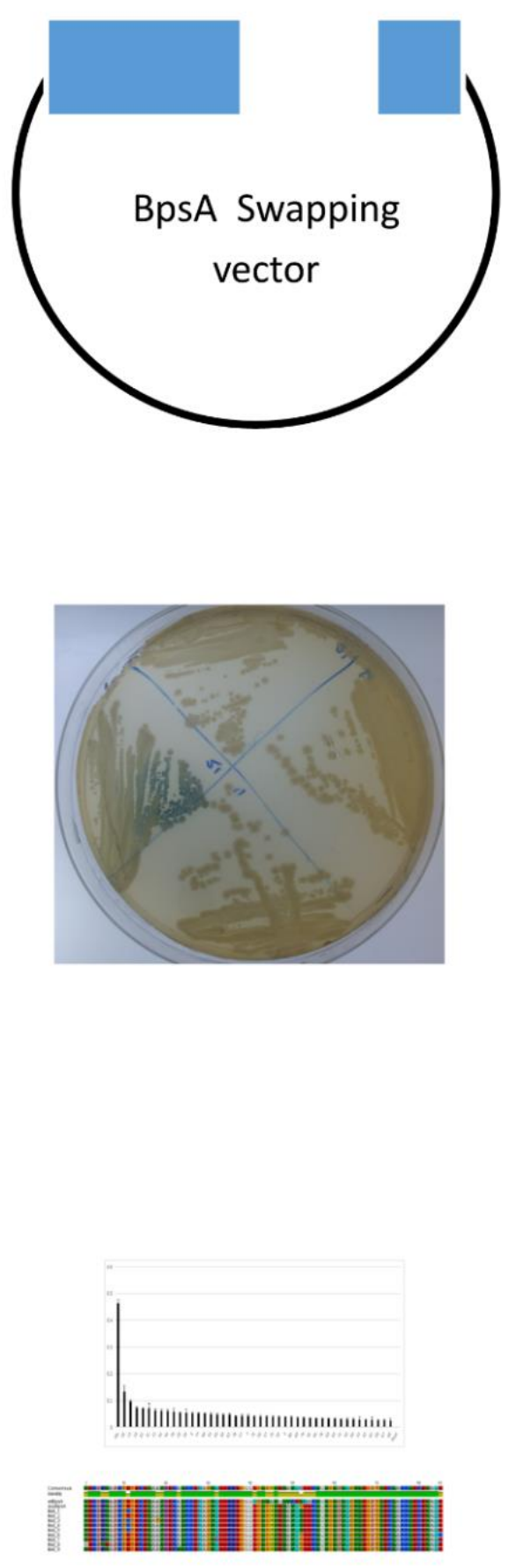

Figure 4.7 Schematic diagram of the directed evolution campaign: (1) The PCP domain is amplified by an error prone polymerase and ligated into the BpsA swapping vector which does not contain a PCP domain; (2) The library is then transformed into BL21 $\Delta$ entD, TB-AcpS expressing cells, and plated on pigment producing plates; (3) Improved mutants that produce detectable pigments more rapidly than other members of the library are re-streaked onto pigment producing plates to confirm activity; (4) Top hits are then qualitatively analysed in liquid culture; (5) The top hits from this step are ranked and their sequences are determined. 


\subsubsection{Library preparation and screening}

The Genemorph Mutazyme $\|{ }^{\circledR}$ kit produced by Agilent Technologies was used to introduce random mutations into each of the three PCP domains at a mutation rate of 3 - 4 nucleotide changes across each domain. The detailed protocol for library construction is described in Section 2.9.

Approximately 40,000 colonies were plated on pigment producing media for each library. The colonies were checked for pigment production every 12 hours. No colonies from the facpBpsA library produced detectable pigment.

Two colonies from the wtBpsA library produced detectable pigment after 48 - 72 hours. When the two wtBpsA variants were retransformed into Bl21 $\Delta$ entD cells expressing TBAcpS, neither produced pigmentation, indicating that another factor other than mutations in the PCP domain was causing the production of pigment. Potentially this could have been caused by a mutation outside of the PCP domain, or mutations to the promotor region of the plasmid resulting in increased levels of BpsA expression.

In contrast, over 200 colonies were recovered from the acpBpsA library that produced detectable pigment. All of the colonies produced indigoidine slowly, with detectable pigment being produced $48-72 \mathrm{~h}$ after induction. The top 100 clones were re-streaked out of pigment producing media, and then induced to confirm activity. From this pool, the 30 clones that developed pigment most quickly were moved into second tier liquid screening (as discussed in detail in Section 2.9.7).

Liquid screening provided a much more quantitative view of the level for each mutant. In the first-tier plate screen colony, location on the plate influences the rate of detectable pigment production. This is due to the uneven diffusion of IPTG through the plate and the competition for nutrients and IPTG from adjacent colonies. Additionally, it relies on identification by the naked eye, which can result in colonies being missed or pigment production not being detected when it first becomes visible.

Despite the improved accuracy of the liquid screen, it is not as sensitive as the first-tier plate screen. None of the top colonies exhibited detectable levels of indigoidine 
production after 48 hours. Instead the nine colonies that most rapidly turned blue on the re-streaked plates were selected and sequenced to identify positive mutations.

\subsubsection{Sequence analysis of the selected first round variants}

Of the nine colonies sequenced, three were identical (Mut_1, Mut_4 and Mut_5, which all had the same mutations, E11K and T52I). It was unclear if these are particularly important mutations, or if they saturated the library early on. The T52I is the only mutation within the second alpha helix. It is also the most common mutation, with three different recovered mutant sequences all containing this mutation. In wtBpsA, the residue at this position is leucine, which is structurally very similar to isoleucine (of note, the codon change in the recovered variants was ACC to ATC, hence the substitution of threonine by isoleucine was achievable by a single base mutation, whereas it is not possible to get from ACC to a leucine-specifying codon without the highly improbable event of two point mutations occurring within the same codon). The alignment of the top recovered variants against unmodified acpBpsA is shown in Fig. 4.8. 


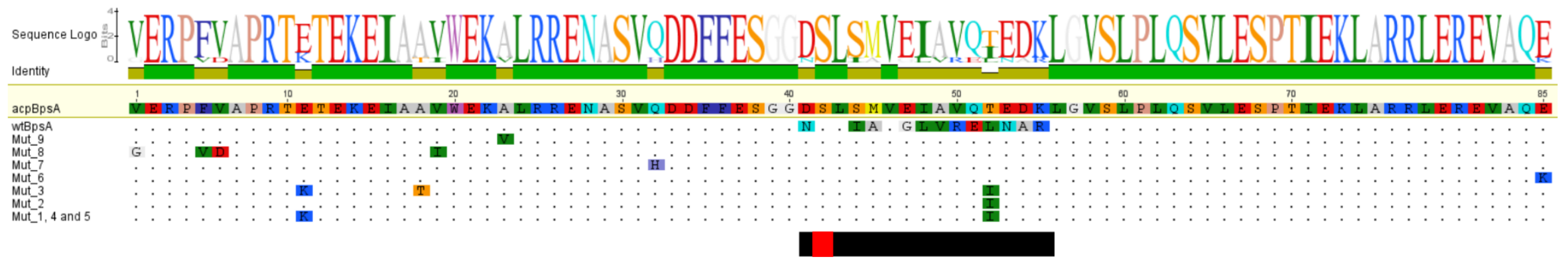

Figure 4.8 Alignment of first round mutant top hits: Multiple sequence alignment of top hits from the AcpM pilot library, the sequence for Mut_ 1 , Mut 4 and Mut 5 have been combined for clarity. The black bar indicates the second alpha helix and the red box highlights the conserved serine residue. The sequence alignment was constructed using Geneious ${ }^{\circledR}$. AcpBpsA is set as the consensus sequence and variants with different residues are identified by their single letter AA code. 


\subsection{Construction and screening of the second round library}

The second round of evolution used the sequences from Fig. 4.8 as a mixed template along with acpBpsA. It was hoped that the variety of sequences would promote DNA shuffling and cause more rapid evolution as beneficial mutations could be combined together and deleterious mutations could be removed (Moore et al., 1997). Each template sequence was added in an equal concentration to avoid biasing the library.

The second round library was constructed in the same manner as the pilot library but was three times the size, with approximately 120,000 clones screened on pigment producing media. The top hits from the library yielded detectable levels of blue pigment after 18 to 24 hours. This was a substantial improvement compared to the first round, where improved colonies were detected 48 to 72 hours after induction. One hundred and twenty top hits were picked for second tier liquid screening.

\subsubsection{Second tier screening of second round library}

The 120 selected variants were screened using the liquid screening method described in Section 2.9.7. The 44 top hits from the liquid culture were then transferred to a master plate (Fig. 4.9B). This master plate was rescreened and the indigoidine production was quantified. The activities of the ten most active hits are displayed in Fig. 4.9A, variants RD2_mut_55 and RD2_mut_13 showing the most increased levels of activity. 
A

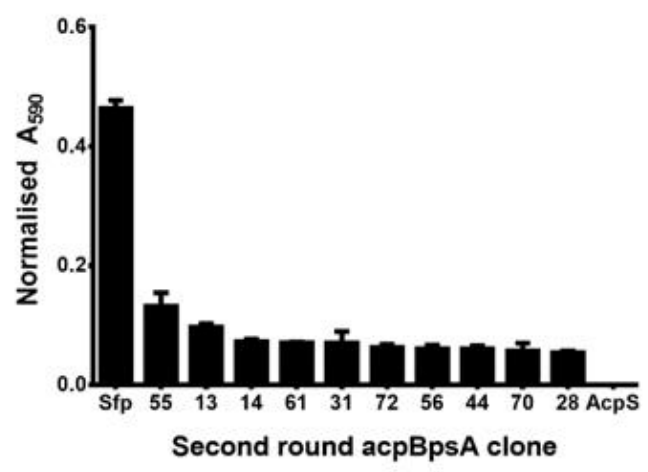

B

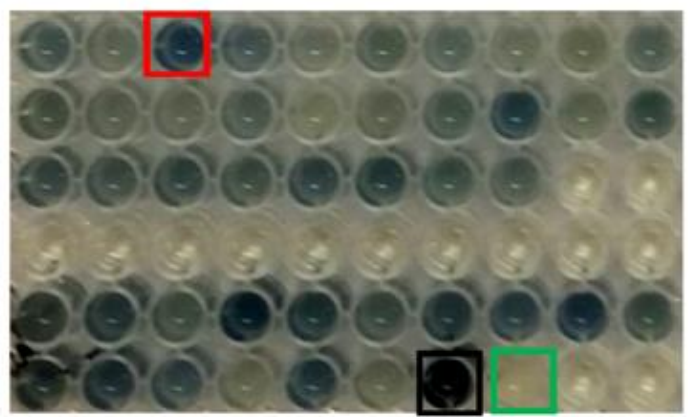

Figure 4.9 Results from second tier screening: (A) The top ten hits from the second round of evolution are graphed in order of their normalised $A_{590}$ value, calculated as described in Section 2.9.8. The positive control (Sfp co-expressed with wtBpsA) had reached saturation by the time the incubation period had been extended enough to detect pigment production by the clones. Data is the average of two biological replicates, each comprising two technical replicates, and error bars indicate \pm SEM. (B) Photo of the round two master plate taken after 48 hours incubation. The red box highlights mutant_55, the top hit from the round. The black box highlights the Sfp positive control and the green box highlights AcpS co-expressed with wtBpsA.

\subsubsection{Sequence analysis of the second round library}

The top ten variants from the second round library were sequenced and then ranked by activity (Fig. 4.10). Three of the variants had the same sequence; RD2_Mut_72, RD2_Mut_70 and RD2_Mut_44, each of which displayed similar levels of activity.

In the top hits from the second round there was a strong enrichment for certain residue changes, indicating that some key substitutions had occurred. For example, all of the top hits other than RD2_Mut_14 contained the E11K substitution, and every variant had the T52l substitution, suggesting that these were both key modifications for improved activity. Two additional sites in the second alpha helix were also mutated in several selected variants, site 47 and site 54 . Interestingly the different between the top performing variant RD2_MUT_55 and RD2_MUT_56, one of the worst-performing variants, was a single amino acid substitution in the former variant at position 47 , which was also within the second alpha helix. 


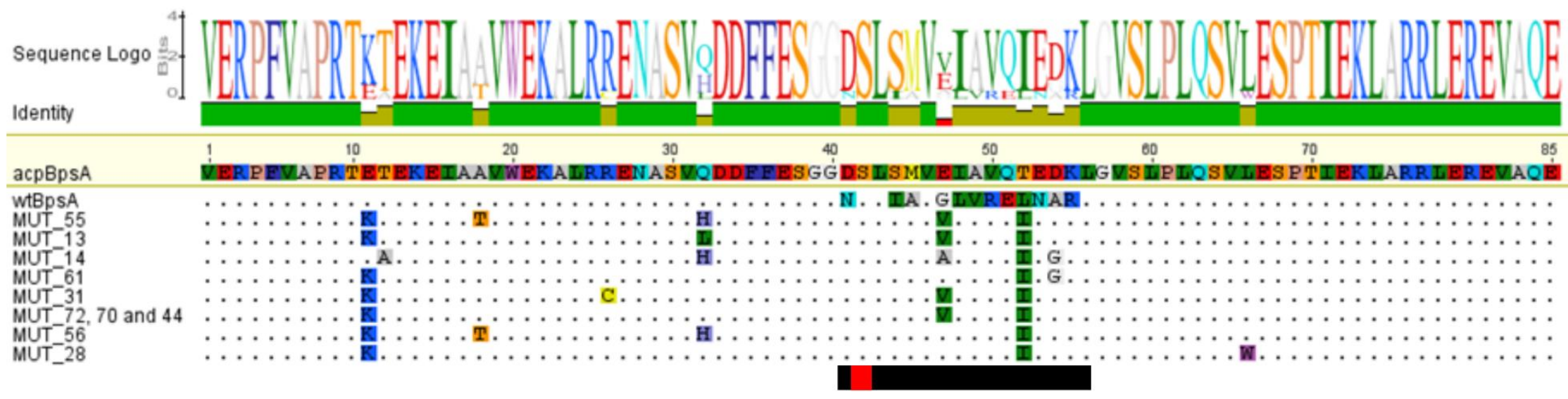

Figure 4.10 Sequence alignment of top selected variants from the second round of directed evolution: Multiple sequence alignment of top hits from the AcpM second round library. The black bar indicates the second alpha helix and the red box highlights the conserved serine residue. The sequence alignment was constructed using Geneious ${ }^{\circledR}$. AcpBpsA is set as the consensus sequence and variants with different residues are identified by their single letter AA code. 


\subsection{Construction and screening of the third round library}

The sequences in Fig. 4.10A were used as a mixed template for the third round library along with both acpBpsA and wtBpsA. These two templates were added to act as a reservoir to help remove deleterious mutations, i.e. by providing a wild-type template for shuffling the original residue back in, should that prove to be more beneficial for activity. Each template was added in equal concentrations to avoid biasing the library. The library was constructed as described in Section 2.9.6, and approximately 100,000 clones were plated on pigment producing media and screened.

Initial hits began to be detected after approximately twelve hours to sixteen hours and were re-streaked and taken into second tier screening. It was noted that the majority of hits from this library didn't appear to be significantly improved over RD2_Mut_55. The two hundred top hits from the third round were then taken into second tier liquid screening.

\subsubsection{Second tier screening of third round library}

Compared to the second-round library, the third-round library had much more uniform amounts of indigoidine production (Fig. 4.11A). The top hits were arrayed onto a master plate to allow for more accurate comparison and re-screened. The top hit from round two, RD2_Mut_55 was also added to the plate as a positive control. Unfortunately, substantial improvements between RD2_Mut_55 and top variants in round three were not observed in the in vivo quantification (Fig 4.11B). The top ten variants from this plate were sequenced and analysed. 
A

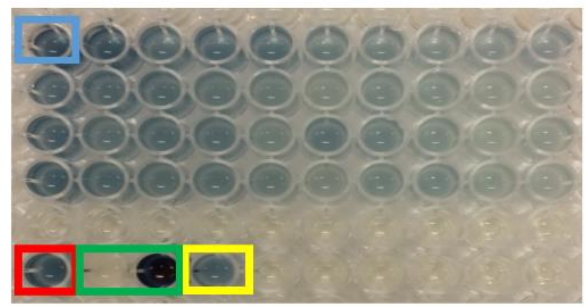

B

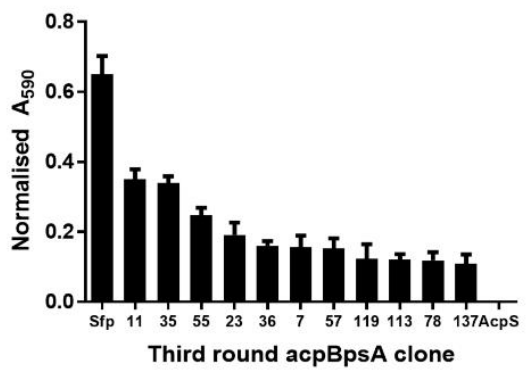

Figure 4.11 Results from third round evolution: (A) Image showing the master plate containing the best performing mutants from the third round of evolution. The blue square is RD3_Mut_35, the yellow square is RD3_Mut_11 and the red square is RD2_Mut_55. The green rectangle highlights the positive Sfp control and AcpS co expressed with wtBpsA. (B) The top ten hits from the third round of evolution are ranked according to normalised $A_{590}$ value, calculated as described in Section 2.9.8. The positive Sfp control had reached saturation by the time incubation period had been extended enough to detect pigment production by the clones. Data is the average of three independent replicates and error bars represent \pm SEM.

\subsubsection{Sequence analysis of the top variants from round three}

The amino acid sequences of the top ten hits from round three were aligned and ranked according to their activity. The sequences are displayed in Fig. 4.12. By this stage there had been a clear enrichment for four key residues: E11K, Q32H/L, and the previous two substitutions noted as occurring within the second alpha helix, E47V/A and T52I. Interesting the two top variants RD3_Mut_11 (T12A) and RD3_Mut_35 (A49T) both contain additional mutations unique to them. For RD3_Mut_35, the substitution A49T occurs within the second alpha helix and this appears to be important for activity as it is the only mutational difference between this variant and RD2_Mut_55. In wtBpsA the wild type residue is a valine and it is unclear why substitution to a threonine led to increased activity. In addition, RD3_Mut_11 contains the substitution S68Y. This mutation was introduced in round three in two different variants. It may be important for the catalytic function of the variants as it appears that RD3_Mut_11 originally came from the RD2_Mut_14 template. 


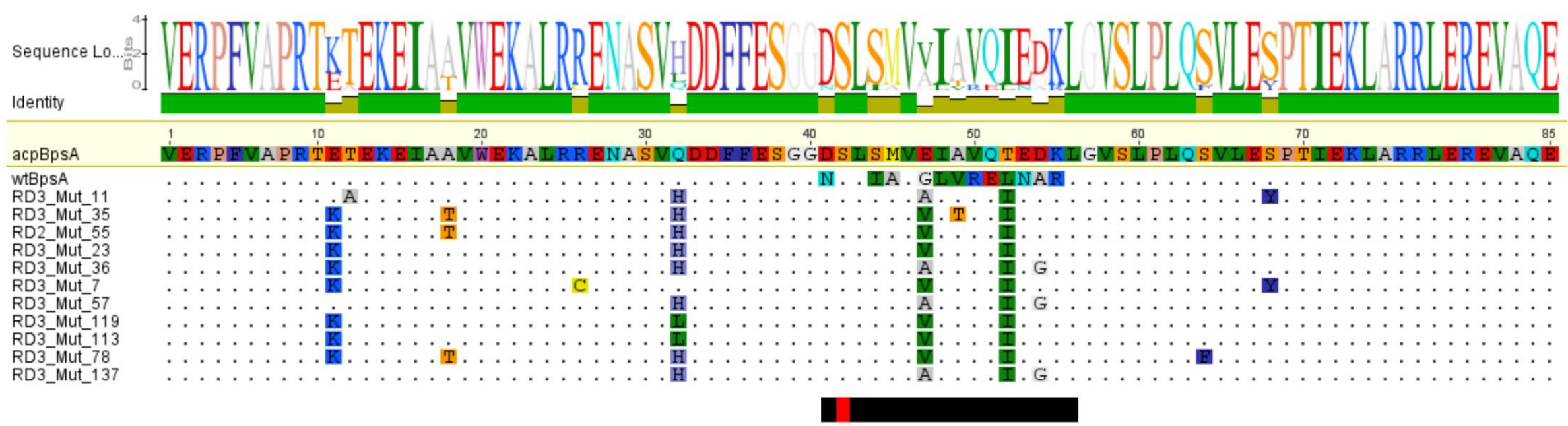

Figure 4.12 Sequence alignment of the top variants from round three: Multiple sequence alignment of top hits from the AcpM third round library ranked in order of activity. The black bar indicates the second alpha helix and the red box highlights the conserved serine residue. The sequence alignment was constructed using Geneious ${ }^{\circledR}$. AcpBpsA is set as the consensus sequence and variants with different residues are identified by their single letter AA code. 


\subsection{In vitro testing of top variants from each round of}

\section{evolution}

After three rounds of evolution, the top three variants from each round were selected for protein purification and analysis, based on either the time taken to show pigment on a pigment producing plate for the first round, or the indigoidine levels detected in second tier screening for the second and third rounds. The selected variants and their sequence identities are listed in Fig. 4.13. Each recombinant plasmid was isolated and then $0.5 \mathrm{ng}$ of DNA was transformed into electrocompetent $\triangle$ entD BL21 cells. A small amount of DNA was used to reduce the chance of double transformants occurring. The cells were then plated on spectinomycin containing plates to select for the clones harbouring the mutant forms of bpsA. Colonies from each plate were then picked and re-streaked on kanamycin containing plates to confirm that they did not contain $\mathrm{pET}$ $28 a(+):: A c p S$.

Unexpectedly, colonies from the second and third round of directed evolution libraries started to produce pigment 48 hours after plating, without being induced by IPTG. This strongly indicated that the mutant forms of BpsA had activity with EC-AcpS in vivo. 


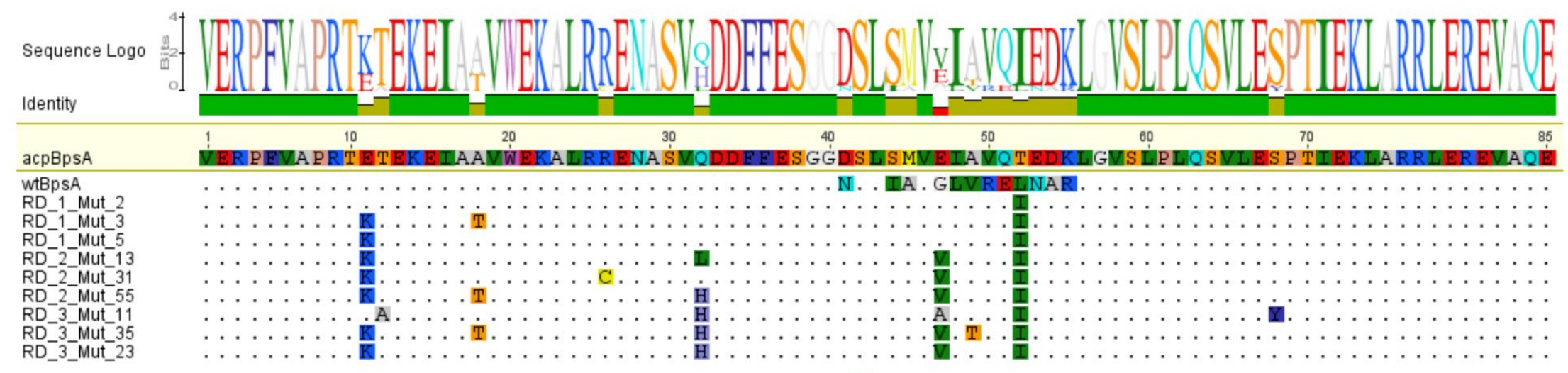

Figure 4.13 Variants selected for in vitro kinetics: Multiple sequence alignment of top three hits from each round. The black bar indicates the second alpha helix and the red box highlights the conserved serine residue. The sequence alignment was constructed using Geneious ${ }^{\circledR}$. AcpBpsA is set as the consensus sequence and variants with different residues are identified by their single letter AA code. 


\subsubsection{Expression and purification of top variants.}

Initial expression and purification tests were performed as described in Section 2.5.

After 16 hours of incubation, substantial amounts of indigoidine was being produced by the cultures (Fig. 4.14). It was noted that the in vivo conversion to holo BpsA would make it difficult to assess if TB-AcpS had any improved activity with the evolved variants of BpsA, as they would already be capable of synthesising indigoidine.

In an attempt to minimise the conversion of BpsA into the holo form by endogenous ECAcpS, a modified expression protocol was used: The culture $\mathrm{OD}_{600}$ at which protein expression was induced was increased from 0.6 to $1-1.2$, and the induction period was decreased from 16 hours to 3 hours. This reduced the amount of indigoidine being produced in the cultures. Using this modified protocol, $400 \mathrm{ml}$ cultures were expressed and purified as described in Section 2.5. As expected with the lower incubation period, yields were lower than with the previous 24 hour induction period (approximately $25 \%$ of the protein yield).

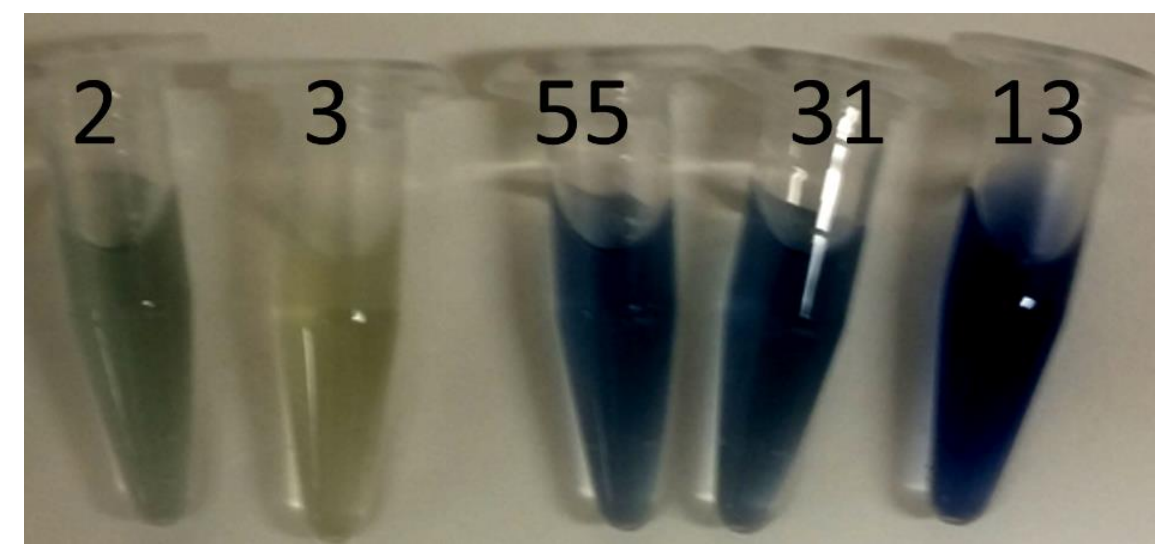

Figure 4.14 Qualitative assessment of indigoidine production following $16 \mathrm{~h}$ by $E$. coli $\triangle$ entD BL21 cells expressing the first and second round variants: Shown is $1 \mathrm{ml}$ of culture containing E. coli $\triangle e n t D$ BL21 containing only the BpsA mutants in the pCDFDuet plasmid. BpsA is being converted into the active holo form by the native EC-AcpS. The photo is taken after 16 hours of expression at $18^{\circ} \mathrm{C}$ post induction with IPTG. Variants Mut_2 and Mut_3 are the top variants from the first round of evolution and variants Mut_55, Mut_31 and Mut_13 are the top variants from the second round. Cultures are not normalised for OD600 values, so do not represent a qualitative value for indigoidine synthesis. 


\subsubsection{In vitro kinetics of variants in their purified form}

To determine the level of conversion to the holo form by the endogenous EC-AcpS, the variants were purified and compared in assays to wtBpsA. Assays were performed as described in Section 2.7.1 except the BpsA variants were assayed at $2 \mu \mathrm{M}$, and $A_{590}$ values were recorded every $30 \mathrm{~s}$ for $1 \mathrm{~h}$. Most of the variants synthesised slightly more indigoidine than the apo-BpsA negative control (red line, Fig. 4.15). This indicated that the evolved variants had been activated in vivo by EC-AcpS. In particular, two variants, RD_3_Mut_35 (dark blue line, Fig. 4.15 ) and RD_3_Mut_11 (brown line, Fig 4.15) from the third round exhibited a substantially faster rate of indigoidine synthesis when compared to apo-BpsA (red line, Fig 4.15). These two variants were the top hits from the third round of evolution indicating that the directed evolution campaign had selected for mutants improved with improved recognition towards EC-AcpS.

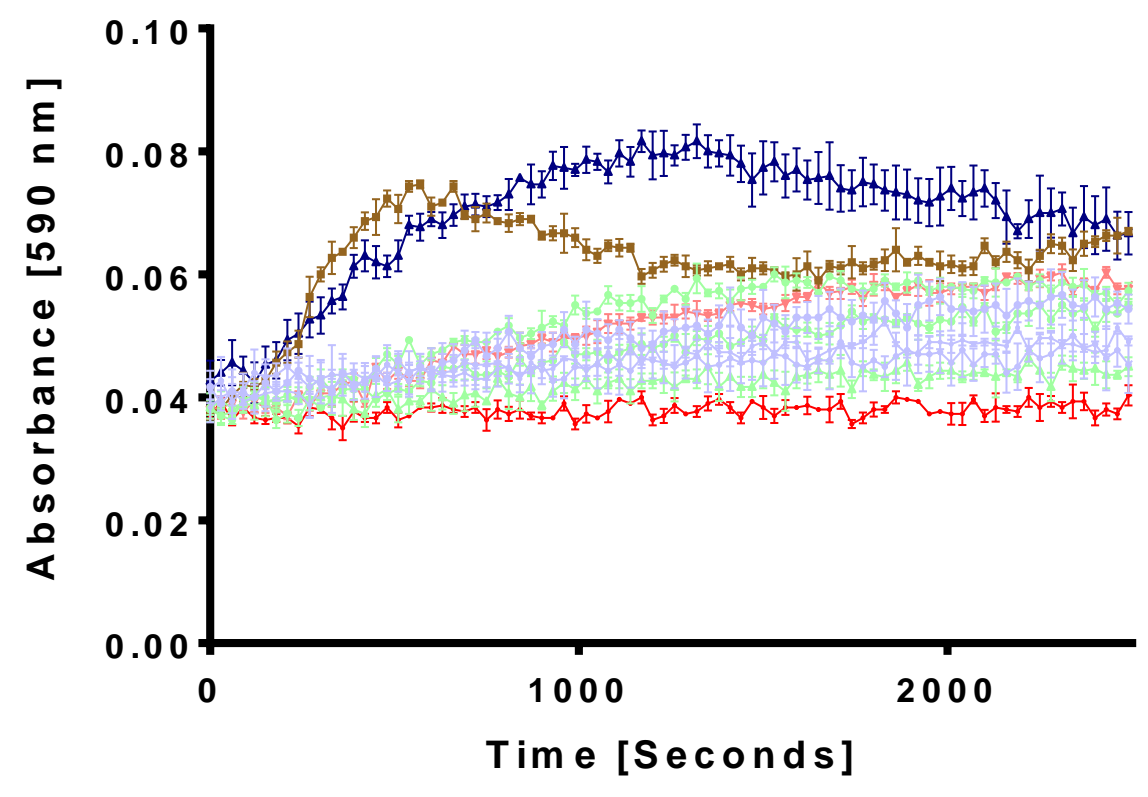

Figure 4.15 Synthesis of indigoidine by acpBpsA variants purified in vitro: Three mutants from each round were assayed to determine the level of conversion to the holo form. Mutants from round one (light blue) and round two (light green) produced little to no indigoidine when compared to apo-BpsA (red line). Two mutants from the third round of evolution; RD3_Mut_11 (brown line) and RD_3_Mut_35 (blue line) produced detectable amounts of indigoidine and displayed the characteristic curve associated with indigoidine synthesis. While the third mutant RD_3_Mut_23 (pale pink line) had a much slower rate of indigoidine synthesis. Data is the average of three replicates and error bars represent \pm SEM. For ease of understanding the individual mutants from each round have been grouped by colour. 


\subsubsection{In vitro kinetics of variants with PPTases}

The apparent gain of activity with E. coli AcpS did not preclude there having also been a gain in activity with TB-AcpS. To determine whether this was the case, kinetic assays were performed as described in Section 2.7.1, with the following modifications: A concentration of $2 \mu \mathrm{M}$ BpsA and $2 \mu \mathrm{M}$ TB-AcpS was used, and $A_{590}$ values were recorded every $30 \mathrm{~s}$ for an hour. The tested mutants showed no improved activity with TB-AcpS when compared to the no PPTase control, suggesting that either all of the variants had already been converted completely to their holo forms during expression in vivo, or that none of the variants had improved activity with TB-AcpS (Fig 4.16).

To test whether the evolved mutants had activity with EC-AcpS in vitro the acpS gene from E. coli W3310 was cloned into pET-28a(+) using the methods described in Section 2.4. The construct was then expressed and purified as described in Section 2.5. EC-AcpS was relatively unstable compared to TB-AcpS and had much lower yields (ca. $5 \mathrm{x}$ less). It also aggregated much more quickly once thawed (not shown).

EC-AcpS was tested against the top variants from each round as described in Section 2.7.1, and did not see an increase in A590 compared to the no PPTase control (Fig 4.16). This suggested that further incubation with an activating PPTase was unable to further increase activity of the evolved acpBpsA variants, in turn suggesting that they had already reached maximal conversion into the holo state. Thus, it appears likely that the limited activity observed in Fig 4.15 may not have been a consequence of only partial activation, but rather, the overall catalytic efficiency of the recombinant BpsA constructs being greatly impaired relative to wtBpsA.

To further confirm if this was the case the variants were tested to see whether they would gain any further activity post-activation with purified PptT. PptT was chosen to test the mutants for two reasons, firstly because it can recognise and activate the PCP domain of wtBpsA rapidly as shown in Chapter 1, and secondly because the second alpha helix of acpBpsA is the native substrate of PptT. Thus, if any Type II PPTase was capable of activating the modified variants it should be PptT. The top variants were incubated with PptT as described in Section 2.7.1, with the following modifications: A concentration of $2 \mu \mathrm{M}$ BpsA and $1 \mu \mathrm{M}$ PptT was used. None of the variants showed any 
noticeable improvement in the rate of indigoidine synthesis when compared to the no PPTase control (Fig 4.16). This provided further evidence that the variants were already completely converted into their holo forms. I therefore concluded that the evolved constructs were greatly impaired in their abilities to synthesise indigoidine relative to wtBpsA.

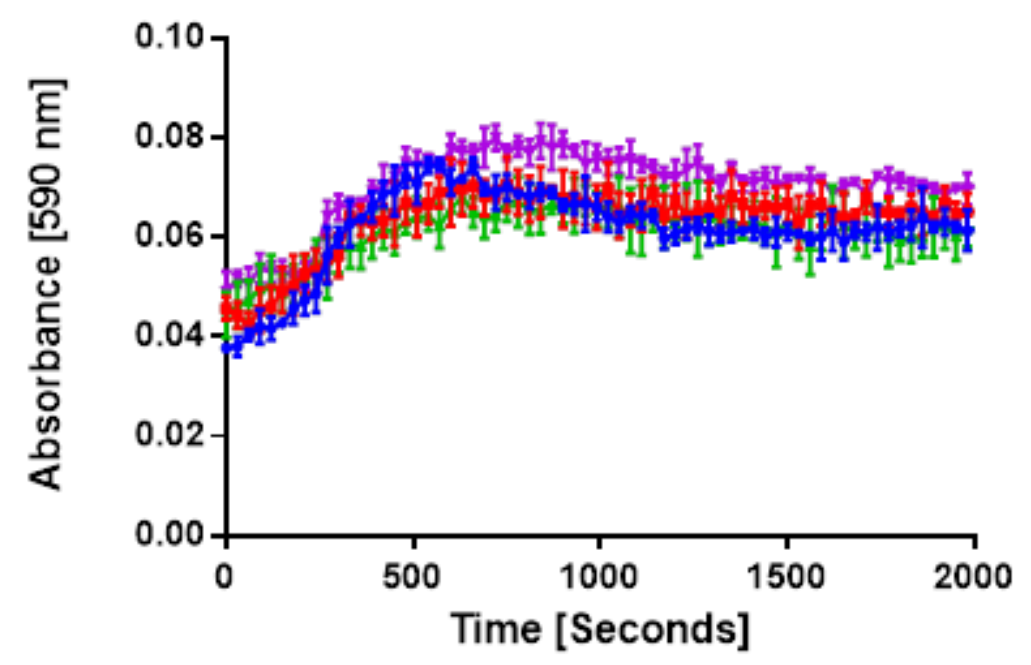

Figure 4.16 Synthesis of indigoidine by RD3_Mut_11 activated by different PPTases: RD3_Mut_11 was incubated with no PPTase (blue line), TB-AcpS (green line), PptT (red line) and EC-AcpS (purple line). No substantial increase in the rate of indigoidine synthesis is observed. Data is the average of three replicates and error bars represent \pm SEM

It remains to be confirmed experimentally whether the variants are indeed their active holo form, e.g. by using Mass Spectrometry or NativePAGE gel and therefore whether this is why we do not see any improvement in the rate of indigoidine synthesis upon addition of EC-AcpS. It is also not yet known whether any of the evolved constructs are also substrates for TB-AcpS as well as EC-AcpS. However, it was decided that further investigation into these issues was not an immediate priority. Supporting the decision to deprioritise this facet of my project, the revelations in the 2015 Zimhony et al paper had by now made it clear by this stage that AcpM was not a native substrate of EC-AcpS; and moreover, there was a lack of certitude around how I might readily avoid the issues of EC-AcpS interfering with the evolution of a TB-AcpS specific form of BpsA. Although 
some ideas around this are presented in the following Discussion section, it was not possible to implement these within the timeframe of my PhD.

\subsection{Discussion}

The work described in this chapter sought to develop BpsA variants with modified PCP domains capable of being recognised and activated by TB-AcpS, and functioning effectively with BpsA. It is highly likely that, due to the selection of the second alpha helix from M. tuberculosis AcpM as an initial template for the directed evolution campaign, the libraries were biased toward EC-AcpS, which can recognise AcpM. As noted above, when the work described in this chapter began AcpM was thought to be post-translationally modified by TB-AcpS (Gokulan et al., 2011), but subsequently this was found to not be the case (Zimhony et al., 2015). Despite the failure to develop a BpsA variant that can efficiently report on TB-AcpS activity, this section of work has shown that it is possible to develop a functional variant that can be converted to the holo form by an AcpS type PPTase, albeit with greatly diminished indigoidine synthesis capacity relative to native BpsA.

\subsubsection{Selection and development of rational designed constructs}

Three initial templates were used to try and develop a variant of BpsA that could be recognised by TB-AcpS, the wtBpsA PCP domain and two modified PCP domains in which the second alpha helix had been substituted by the second alpha helix from $M$.

tuberculosis FAS-1 or AcpM. In addition to the problem with AcpM turning out not to be a native substrate of TB-AcpS, an issue with the selection of the FAS-1 second alpha helix might be the residue present at the +4 position (relative to the invariant serine where the Ppant arm is attached). The +4 residue is known to be important for interacting with the TE domain of BpsA and recombinant constructs have been shown to function best when there is a hydrophobic residue in this position (Owen et al., 2016). In the FAS-1 second alpha helix this residue is asparagine. In contrast, this residue is valine in the AcpM second alpha helix, which is the same residue present in the PCP domain of BpsA. If the initial generation of an error prone PCR library from the facpBpsA genetic template did not modify this residue to a hydrophobic residue, this may have prevented recovery 
of hits from this library. For future evolution projects, it might prove advantageous to rationally modify the +4 residue to a valine prior to epPCR amplification of the template.

\subsubsection{Directed evolution of acpBpsA}

Directed evolution has been used previously with BpsA to improve activity between PPTases and the BpsA PCP domain and has met with success, with the most active evolved variant in the Owen et al (2016) study having $75 \%$ of wtBpsA activity restored in vivo from a non-functional enzyme. However, in vitro this variant had a maximum indigoidine synthesis velocity only $6.5 \%$ that of wtBpsA (Owen et al., 2016).

Nevertheless, this result, together with the finding of Finking et al (2004) that a single residue change was enough to allow AcpS to interact with the PCP domain (Finking et al., 2004), indicated that only one or two mutational changes might be sufficient to evolve a functional variant of BpsA that was capable of recognising TB-AcpS and was sufficiently active to enable screening for TB-AcpS inhibitors.

The pilot libraries served two purposes. Firstly, to try and identify which of the three templates (wtBpsA, acpBpsA and facpBpsA) was the most likely to be functional as only wtBpsA had shown very low levels of activity with TB-AcpS. It was hoped that one or two mutations would be enough to produce detectable levels of activity with TB-AcpS. This was found to be the case for the acpBpsA construct, with only one or two mutations seemingly being enough to restore activity in vivo (i.e., one or more of $\mathrm{E} 11 \mathrm{~K}, \mathrm{Q} 32 \mathrm{H}$ or T52I). Interestingly out of these substitutions, T52I is the only one located within the second alpha helix (Fig. 4.8). Secondly, starting a larger directed evolution campaign with a more diverse range of templates would hopefully increase the speed of evolution and reduce the chance of hitting an evolutionary bottleneck.

Four mutations appeared to have a critical function either in improving the catalytic efficiency or recognition between acpBpsA and type I PPTases. Two of these residues glutamic acid at position 11 and glutamine at position 32 are outside of the second alpha helix. Interestingly, RD3_Mut_11 does not have the common lysine substitution at position 11, instead it has a T12A substitution, which may have a similar effect. Two mutations within the second alpha helix appear to be essential. All mutants from the second round of evolution onwards that were sequenced possess the T52I mutation 
indicating it is necessary for improved function. The other common mutation that was found in all sequenced round three variants was E47A/V. In wildtype BpsA this residue is glycine.

It is unknown to what extent these mutational changes drive activity with EC-AcpS rather than TB-AcpS, however there is some independent evidence that some of them may assist with overall PCP domain functionality when the second alpha helix is modified. Concurrent with this evolution campaign, a Masters student in the Ackerley Laboratory, Jack Sissons, was conducting a similar evolution campaign with the PCP domain of BpsA. His project focused on developing PPTase-recognition tags based on the second alpha helix of a native PCP domain recognised by the Type II PPTase EntD from E. coli. Jack's project took the second alpha helix from the E. coli EntF PCP domain and inserted it into the PCP domain of BpsA in a similar manner to that described in Section 4.4 (Sissons, 2017). The constructs were then evolved to have improved activity with EntD. Surprisingly, despite the different PPTase types and second alpha helix sequences used, Jack also found a saturation of E11K and Q32H residue changes in his improved variants (Sissons, 2017).

To investigate this convergence, Jack constructed and tested two variants bearing either the $\mathrm{E} 11 \mathrm{~K}$ or the $\mathrm{Q} 32 \mathrm{H}$ substitutions, to see if either of these enhanced the activity of BpsA. It was found that neither mutation enhanced the binding activity between EntD and BpsA or the activity of wtBpsA itself. In fact, both mutations were slightly detrimental to synthesis of indigoidine by BpsA (Sissons, 2017). This suggests that these two mutations may have a role in allowing the PCP domain to accept foreign alpha helices at the helix two position, rather than improving recognition between the PPTase and PCP domain, or overall catalytic function.

\subsubsection{Critical evaluation of the directed evolution process}

While substantial improvements in in vivo activity were achieved through the directed evolution campaign, the gains in in vitro activity appeared less impressive. All of the purified mutants, when incubated with PptT (the native PPTase for activation of AcpM) exhibited no further increase in activity over their purified form, indicating that they had likely been purified in a near-complete holo state. This further implies that although the 
recognition between PPTase and PCP domain may have been improved, the overall catalytic activity of the enzyme had been substantially diminished.

A major cause of this could be due to asking the rationally designed constructs to try and do too much at once. During the evolution campaign there are two selections present for the constructs. Firstly, the interaction between AcpS and the modified PCP domain; and secondly, the overall catalytic function of the BpsA mutant. Since the in vivo selection occurs over an extended time frame it means that for the primary selection is likely to be for improvement in recognition between AcpS and the modified PCP domain rather than overall catalytic function. This could be why the enzymes appeared to have relatively poor activities in vitro.

The substitution of the second alpha helix by an ACP-derived helix represents a significant change in amino acid sequence in an area that is critical to the function of the PCP domain, and it may simply require more changes than are achievable using a stepwise error prone PCR approach to restore functionality, especially if several residues in the second alpha helix need to be modified simultaneously to achieve an effect.

Another key issue with the evolution strategy was the presence of the endogenous ECAcpS within the host $E$. coli cells. This PPTase is essential and cannot easily be knocked out of E. coli. It was hoped that it would be present at low enough of a level in vivo not to exert an effect on the evolution of acpBpsA. I hoped to further reduce the chance of applying the wrong selection pressure by strongly over-expressing the TB-AcpS, expecting that this would outcompete EC-AcpS, and hence minor gains in recognition by TB-AcpS would lead to substantial improvements in indigoidine synthesis. As far as I was able to tell, this strategy did not work - certainly, it did not prevent recognition of the recombinant constructs by the endogenous EC-AcpS.

In order to avoid interference by endogenous PPTases it may be necessary to attempt to knockout the E. coli acpS and replace it with a chromosomally integrated $M$. tuberculosis acpS. A similar strategy has been shown to be possible previously in $P$. aeruginosa where the endogenous PcpS was knocked out and the strain remained viable with a chromosomally integrated acpS (Barekzi et al., 2004). However, in E. coli this strategy may not be possible as TB-AcpS has some structural dissimilarities to EC-AcpS 
(Dym et al., 2009) and may not be able to activate the essential ACP domains of E. coli. Nevertheless, if a second evolution campaign is undertaken this would likely be essential for in vivo evolution within an E. coli host strain to be possible. An alternative to this might be to move the evolution to a more similar species to $M$. tuberculosis, for example M. smegmatis. However, this would come with a range of issues, including lower transformation efficiency and growth rates. There is also no guarantee that BpsA would be functional in M. smegmatis, although this could be easily tested.

\subsubsection{Additional improvements to the experimental procedures in the chapter}

Due to the failure of the experimental work in this chapter to improve activity between TB-AcpS and BpsA I considered it cogent to discuss in detail additional possible improvements to the experimental methods in this chapter. For the other experimental chapters, critical evaluations are primarily considered from a more global perspective in Chapter Seven.

The rational design and selection of an initial PCP construct appears to be the critical step for a successful evolutionary campaign, as the majority of the issues found during the evolutionary process stemmed from the unsuccessful use of the incorrect second alpha helix. Unfortunately to the best of my knowledge no analysis has been undertaken of the interactions between TB-AcpS and FAS-1, so it is difficult to know exactly what residues in the ACP domain are critical for this interaction. It is possible that the swap of the entire second alpha helix may be too large a change to be tolerated since the unevolved facpBpsA construct exhibited no indigoidine synthesis either in vitro or in vivo. Nevertheless, since only a single round of evolution examining only 40,000 clones was completed here, it would be premature to conclude that restoration of activity is not readily achievable for this construct.

Using BpsA as a reporter to identify the key residues in the recognition of a chimeric PCP-domain by TB-AcpS may not be the best starting point, due to interference between EC-AcpS and the modified BpsA variant (unless EC-acpS could be knocked out and replaced by TB-acpS, as suggested above). An alternative approach could be to use a phage display library such as the one discussed in Section 1.8. This would allow the rapid 
screening of a large library that could be comprised either of the entire BpsA PCP domain with residues in the second alpha helix modified via guided random mutagenesis, or else just the second alpha helix region, using a method similar to that of (Zhou et al., 2007)

Once recognition between TB-ACPS and a modified PCP domain had been confirmed, the second phase of evolution could occur. This would focus on the improvement of the catalytic efficiency of BpsA. Since the modified PCP domain would already be known to be a substrate for TB-AcpS, the likelihood of interference by EC-AcpS would be reduced. This study has already identified two mutations (E11K and Q32H) that appear important for overall PCP function, and the rational incorporation of these mutations into improved variants identified through the use of phage libraries or targeted mutagenesis might prove useful in accelerating the evolutionary process.

During the three rounds of directed evolution described in Sections 4.5-4.7, it was not considered that the endogenous EC-AcpS might interfere with the evolutionary outcome. In the future, it would be essential to test a range of mutants for in vitro activity with TB-AcpS at the completion of each cycle, rather than assuming that activity had been specifically improved with this PPTase. Another method that could be useful at this step might be to use TB-AcpS to attach fluorescent CoA analogues to the PCP domain or BpsA itself (Gokulan et al., 2011), as this would ensure selected variants are capable of recognising the modified PCP domains.

Finally, it is important to note that the in vivo activation of the BpsA variants by EC-AcpS may not be an unsurmountable problem. Work by Rothmann et al (2014) has shown that the acyl carrier protein hydrolase (AcpH) family of enzymes can be used to rapidly remove the Ppant arm from CP domains (Rothmann et al., 2014). In the future, evolved variants could be treated with $\mathrm{AcpH}$ to remove the Ppant arm and then tested in vitro for activity with TB-AcpS. It should not be assumed simply because an evolved variant is activated by EC-AcpS, that it cannot also be activated by TB-AcpS.

Despite the failure to evolve variants of BpsA that can act as effective reporters on TBAcpS activity, the research described in this chapter has nevertheless proven that BpsA can be used to evolve PCP domains that are substrates for AcpS type PPTases. It has also 
identified key residues that may be important for improving the acceptance of modified second alpha helices. 


\section{Chapter 5- Development of a biosensor to quantify L-glutamine}

\subsection{Publications}

Brown, A. S., Robins, K. J., \& Ackerley, D. F. (2017). A sensitive single-enzyme assay system using the non-ribosomal peptide synthetase BpsA for measurement of Lglutamine in biological samples. Scientific Reports, 7, 41745. doi:10.1038/srep41745

Ackerley, D. F., Brown, A. S., \& Robins, K. J. (2015). Methods of detecting and measuring glutamine and analogues thereof, and methods related thereto. PCT patent filing WO/2015/084189, published $11^{\text {th }}$ June 2015

\subsection{Chapter summary}

The ability to rapidly, economically and accurately measure L-glutamine concentrations in biological samples is important for many areas of research, medicine and industry, however there is room for improvement on existing methods. This chapter focuses on the development a novel biosensor to detect and quantify L-glutamine levels in a variety of scientifically or clinically relevant conditions. BpsA converts two molecules of Lglutamine into the easy to detect blue pigment indigoidine. The direct relationship between the amount of L-glutamine consumed and the level of indigoidine produced can be used to accurately measure L-glutamine in biological samples.

BpsA, like all NRPSs, exists in either of two forms, an inactive apo form or an active holo form. Due to the mild antimicrobial properties of indigoidine it is difficult to express and purify in its active form. A new method of purification was developed which takes advantage of the endogenous levels of co-enzyme A present in the $E$. coli cell lysate and resulted in consistent high yields of holo-BpsA.

Indigoidine has low solubility in aqueous solutions, which hindered early attempts to develop a protocol for creating accurate standard curves. Despite attempts to modify and improve the protocol, direct kinetic measurement of indigoidine synthesis was found not to be a reliable method for the detection and quantification of L-glutamine. 
Instead a new method employing resolubilisation in the solvent DMSO was developed. This method was optimised and tested for a variety of samples including blood and urine. The optimised protocol can readily detect and accurately quantify L-glutamine. The long-term stability of BpsA was characterised and the protocol was found to offer advantages over commercially available glutamine quantification kits.

\section{2 introduction}

\subsubsection{The importance of L-glutamine as a biomarker}

Glutamine is the most abundant amino acid in the human body, owing to its ability to act as the major intercellular transporter of amino-nitrogen and as a key fuel source for rapidly dividing cells, including cells of the immune system and intestinal lining. Consequently, glutamine is very important in numerous aspects of medicine. Not only is it used as a common supplement for athletes or patients experiencing critical illness but abnormal levels in bodily fluids can be symptomatic of certain diseases, e.g. metabolic diseases (Serrano et al., 2011; Trinh et al., 2003) over-training syndrome (Agostini and Biolo, 2010; McKenzie, 1999) or neurodegenerative disorders (Chen and Herrup, 2012; Tsuruoka et al., 2013) . Moreover, tumours can become dependent on glutamine as an energy source (Wise and Thompson, 2010), and this can manifest as unusually low glutamine levels in serum or saliva (Ikeda et al., 2012; Tan et al., 2013).

Glutamine is also enormously important from a research perspective as it provides a particularly useful energy source for mammalian cell culture (Eagle et al., 1956). However, excessive levels of glutamine in culture medium can inhibit the transport of other amino acids (Yang et al., 2010) or result in the generation of toxic levels of ammonia (Doyle and Butler, 1990; Tritsch and Moore, 1962). For all of these reasons and more, it is important to be able to accurately quantify the levels of glutamine that are present in complex biological mixtures.

\subsubsection{Current detection methods}

The "gold standard" for glutamine measurement, and method most commonly employed by hospital laboratories, is HPLC. However, this method is expensive, unwieldy, and unsuited to rapid turnaround of samples. Specialized instruments have 
been developed to monitor the concentrations of various metabolites including glutamine in cell culture and fermentation media, but these instruments are also expensive and a typical device was recently found to be insufficiently accurate for pointof-care analysis of glutamine levels in patient plasma samples.

In contrast, enzymatic methods offer promise for rapid, accurate and cost-effective quantification of glutamine in diverse biological samples. The primary method that has been used to achieve this, employed in a wide range of commercially available kits, is a two-step enzymatic conversion of glutamine to glutamate by glutaminase followed by a further deamination of the glutamate to $\alpha$-ketoglutarate by glutamate dehydrogenase (Lund, 1986). As the second step is accompanied by a proportional reduction of $\mathrm{NAD}^{+}$to $\mathrm{NADH}$, the reaction can be monitored spectrophotometrically. However, not only does this method involve multiple reagents and time-consuming reaction steps, it also requires that the baseline of glutamate in the sample be established separately, so that this can be subtracted from the combined (glutamine + glutamate) measurement. This extra step introduces additional error and potential for cross-sample contamination.

\subsubsection{Using BpsA as a biosensor for the detection of L-glutamine}

Here I sought to develop an alternative means for assaying L-glutamine, based on a single enzymatic conversion of two molecules of L-glutamine into the directly detectable blue pigment indigoidine (Fig. 5.1), by BpsA. Despite the many different applications of BpsA that have previously been investigated, at the start of this work no one had previously reported adaptation of BpsA for measurement of glutamine, most likely because the unusual and poorly understood solution chemistry of indigoidine results in unusual absorbance profiles (Fig. 5.2A) from which it is not obvious how a linear standard curve might be generated. Indeed, a subsequent PCT patent application that was filed and published by another group (Tao, 2015) shortly after our own PCT patent publication (Ackerley et al., 2014) failed to overcome this problem and instead relied on the direct measurement of the maximum value of indigoidine absorbance. Here I demonstrate that is not a reliable method for accurate L-glutamine measurement, and show that a final solubilisation step can effectively resolve this issue. 


\section{$2 \times$ L-glutamine holo-BpsA indigoidine}
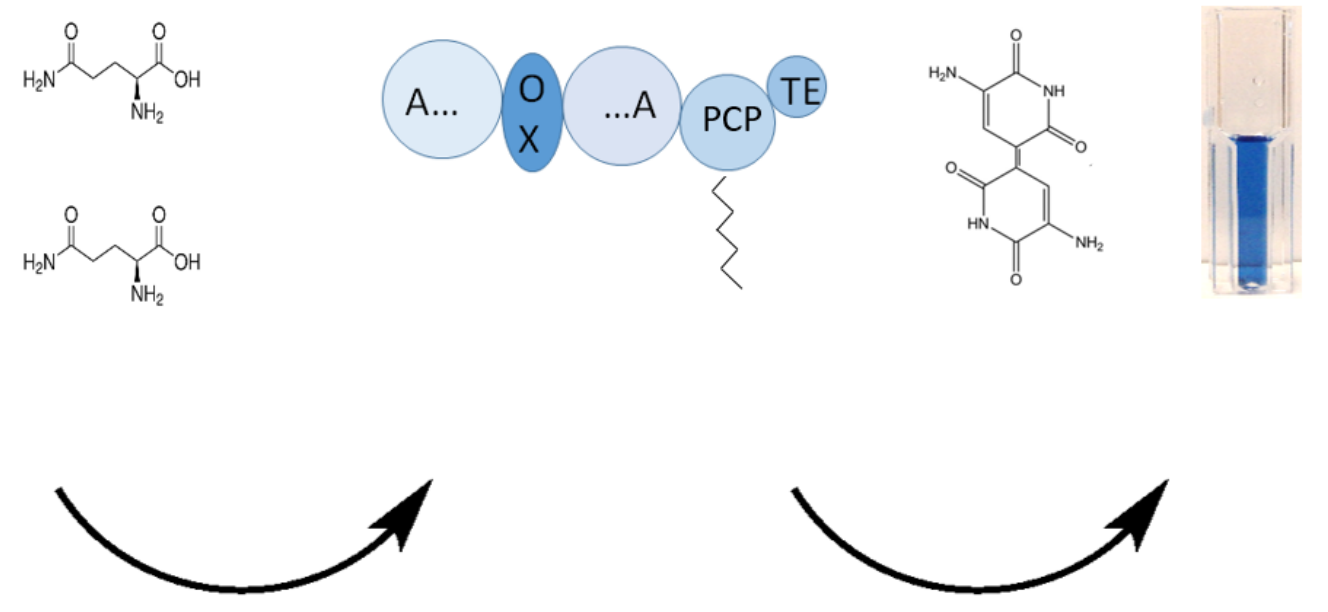

Figure 5.1 Schematic diagram showing two molecules of L-glutamine being converted into one molecule of the blue pigment indigoidine by holo BpsA: Two molecules of L-glutamine are converted into a single molecule of the bright blue pigment indigoidine in an ATP powered reaction by holo BpsA.

\subsection{Aims of the research in this chapter}

- To develop a method to reliably express and purify holo-BpsA

- To develop a method to create reproducible and accurate standard curves of Lglutamine.

- To accurately measure L-glutamine concentrations in a variety of media types and clinically relevant fluids.

\subsection{Expression and purification of holo-BpsA}

Like all NRPS enzymes, in order for BpsA to synthesise its product (i.e., indigoidine) it first needs to be converted from the inactive apo form to an active holo form. This conversion is achieved by the attachment of a Ppant moiety derived from coenzyme $A$ to the peptidyl carrier protein domain (PCP) of BpsA. This reaction is catalysed by a PPTase enzyme. 
Ppant attachment is difficult to achieve in vivo prior to BpsA purification, as the mild anti-bacterial properties of indigoidine inhibit the growth of $E$. coli cells that are producing holo-BpsA (Beer et al., 2014). Therefore, it was necessary to develop a method to activate BpsA post-expression.

\subsection{1 in vitro conversion of BpsA from the apo to the holo form}

Initial work used the method described in Owen et al (2012), with both the activating PPTase and apo-BpsA being purified independently (Owen et al., 2012). A master mix containing apo-BpsA, PcpS, a buffering solution, Co-enzyme $\mathrm{A}$ and $\mathrm{MgCl}_{2}$ was incubated for $20 \mathrm{~min}$ at $30^{\circ} \mathrm{C}$ to bring about the conversion to the holo form. This system was impractical for several reasons. Firstly, it was both expensive and time consuming. A significant excess of Co-enzyme A was essential (the protocol called for $100 \mu \mathrm{M}$ CoA to activate $3.4 \mu \mathrm{M}$ of BpsA). Both the PPTase and BpsA had to be purified independently increasing the time taken for preparation of holo BpsA. The method also required significant amounts of nickel affinity resin to be used along with large volumes of culture (typically $800 \mathrm{ml}$ for the PPTase and $400 \mathrm{ml}$ for BpsA). The level of phosphopantetheinylation activity was also variable as the PPTases are unstable once purified from the native cell lysate. In order to overcome the time and cost associated with this approach a new method was developed.

\subsubsection{In-lysate conversion of BpsA from the apo to the holo form}

To avoid production of holo-BpsA in vivo, BpsA was expressed as a $6 \mathrm{His}$-tagged protein in a strain of E. coli BL21(DE3) that had the endogenous non-essential PPTase gene entD knocked out as previously described (Owen et al., 2012). Prior to purification, conversion of 6 His-tagged apo-BpsA to the holo form was achieved by mixing the soluble fraction of cell lysate with lysate from E. coli BL21 cells that were over-expressing a 6His-tagged PcpS, together with excess coenzyme $A$ as a source of PPT. Initially both PcpS and BpsA were expressed in vectors containing a $6 \mathrm{His}$ tag. To remove the PcpS from the lysate once it had attached the PPT moiety to BpsA I sought to take advantage of its inherent instability. The combined lysates were incubated at $25^{\circ} \mathrm{C}$ for two hours and then pelleted at 26,000 $\mathrm{g}$ for $20 \mathrm{~min}$ to remove the aggregated PcpS. While this method worked reasonably well and resulted in purified holo-BpsA the purification process took longer as the $100 \mathrm{kDa}$ cut off column was getting clogged by PcpS. Instead, to ensure 
that PcpS had been completely removed during the purification process a vector was developed which did not contain a 6 His tag.

\subsubsection{Construction and testing of pNOHISPET}

pET-28a (+) is a common bacterial expression vector developed by Novagen ${ }^{\circledast}$. It contains both an $\mathrm{N}$-terminus $6 \mathrm{His}$ tag and a $\mathrm{C}$-terminus $6 \mathrm{His}$ tag. There is an Ncol restriction site upstream of the $\mathrm{N}$-terminus $6 \mathrm{His}$ tag which allows genes to be cloned in without attaching the $6 \mathrm{His}$ tag. However, it was not possible to take this approach with $p c p S$ as the gene contains an internal $\mathrm{Ncol}$ site. Instead, a novel vector without an $\mathrm{N}$-terminus 6 His tag based on pET28a was developed which would facilitate the expression of proteins without a $6 \mathrm{His}$ tag.

pNOHISPET was created by digesting pET-28a(+) with Ncol and Sall, followed by bluntending of the digested plasmid using T4 polymerase and circularisation using T4 DNA ligase. The construct was then sequence verified to ensure cloned genes would remain in frame. NOHISPET still had the majority of the multiple cloning site present, to enable cloning of $p c p S$ and expression of PcpS without a $6 \mathrm{His}$ tag. The $p c p S$ gene was then cloned into the construct using the HindIII and Xhol restriction sites.

Lysates containing apo-BpsA and PcpS without a $6 \mathrm{His}$ tag were mixed and incubated for two hours with additional CoA added. The mixed lysate was then purified via nickel affinity chromatography as described in Section 2.5 , and activity was confirmed by monitoring indigoidine production in the presence of L-glutamine and ATP (Fig. 5.2A). To confirm that the majority of the BpsA had been converted to the active holo form during the co-incubation with PcpS, BpsA was re-incubated with purified PcpS and the reaction velocity was measured as described in Section 2.10. Further incubation of this enzyme with 6His-tagged PcpS that had been purified separately did not lead to a statistically significant increase in the rate of indigoidine synthesis (Fig. 5.2B). Although 100\% conversion to the holo form is not essential for an assay system where all samples are tested using the same enzyme preparation, this observation nevertheless suggests that near-complete conversion of BpsA to the holo form had been achieved during the mixed lysate incubation step. 

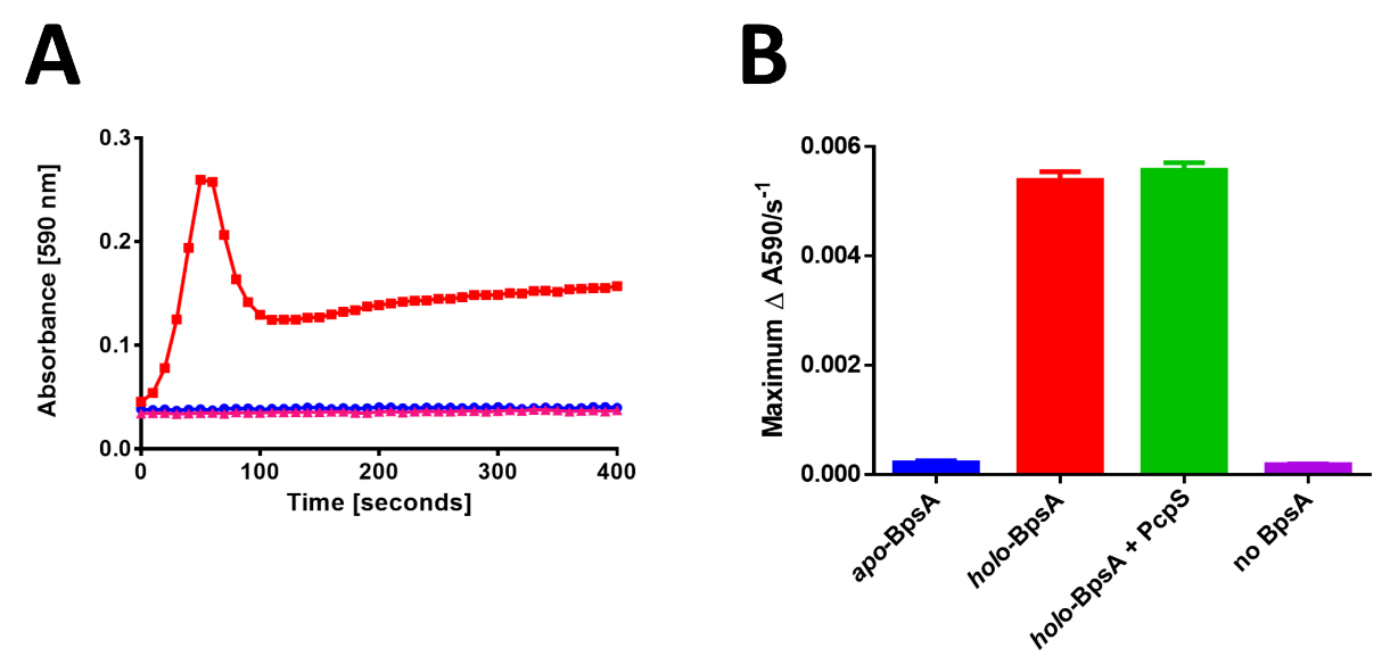

Figure 5.2 Conversion of BpsA to the holo form using PcpS: (A) Holo-BpsA (red circles) is able to rapidly synthesise indigoidine, while apo-BpsA (blue) is unable to synthesise indigoidine and has the same absorbance readings as the no BpsA (purple) negative control. Data are the mean the values from two independent experiments each comprising of three technical replicates and error bars indicate \pm SEM. (B) Incubation of holo-BpsA with purified PcpS and Coenzyme $A$ does not increase the reaction speed. Data are the mean value of three technical replicates and error bars indicate \pm SEM.

\subsection{Development of an assay to quantify indigoidine production}

As noted in Section 1.11.4 indigoidine synthesis in an aqueous solution does not yield an asymptotic curve of absorbance over time. Instead of being a smooth curve that levels off as a maximum $A_{590}$ value is approached, a typical indigoidine synthesis curve rapidly reaches a first maximum, after which the $A_{590}$ declines equally rapidly before slowly increasing once again (Fig. 1.3, Fig. 5.2A). Although greater starting concentrations of Lglutamine in a test solution result in higher initial $A_{590}$ maxima (Fig. 5.3A), I found that plotting these maxima from samples containing a range of known L-glutamine concentrations yielded a curved rather than linear series of points (Fig. 5.3B). Plotting the initial maximal rates of indigoidine synthesis (i.e., the steepest slope of each individual curve) was sometimes capable of yielding a linear set of values suitable for generation of a standard curve, however this outcome was not reproducible at different holo-BpsA concentrations, and I considered that the kinetic nature of the assay was more prone to variability than an end-point assay would be. 
A

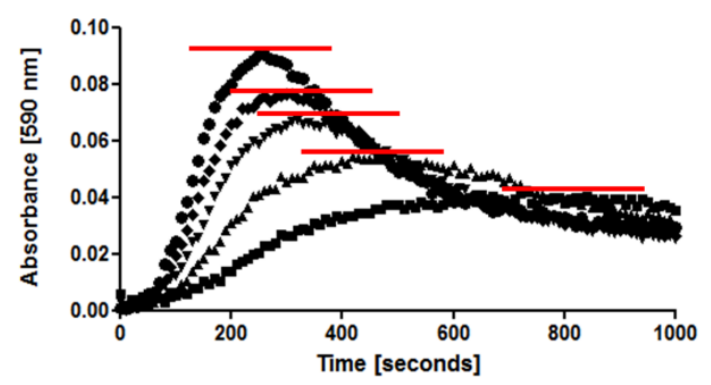

B

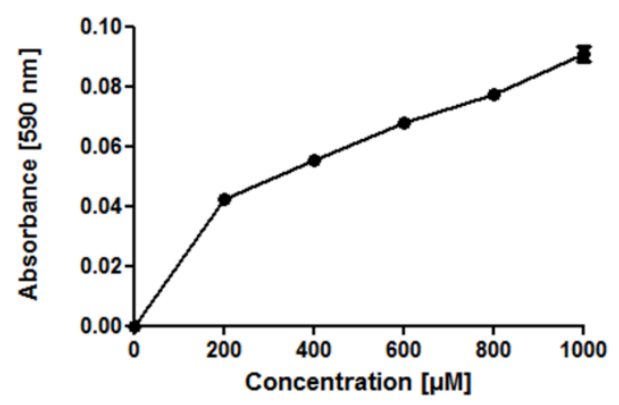

Figure 5.3 Direct monitoring of indigoidine synthesis does not yield a standard curve: (A) To initiate the reaction, 10 $\mu \mathrm{L}$ of L-glutamine stock solutions were added to the following concentrations: $1000 \mu \mathrm{M}(\bullet), 800 \mu \mathrm{M}(\bullet), 600 \mu \mathrm{M}(\boldsymbol{\nabla})$, $400 \mu \mathrm{M}(\mathbf{\Delta}), 200 \mu \mathrm{M}(\mathbf{\square})$ or $0 \mu \mathrm{M} \mathrm{L-glutamine} \mathrm{(omitted} \mathrm{from} \mathrm{the} \mathrm{graph),} \mathrm{and} \mathrm{A}_{590}$ values were recorded for each well every ten seconds. The data has been normalised to zero for the average $0 \mu \mathrm{M} \mathrm{L-glutamine} \mathrm{standard} \mathrm{value.} \mathrm{Each} \mathrm{data}$ point is the average of three technical replicates. The red lines mark the peak absorbance value observed for each Lglutamine concentration. (B) A standard curve was generated from the peak absorbance values recorded for each Lglutamine concentration. The data has been normalised to the zero for the $0 \mu \mathrm{M} \mathrm{L-glutamine} \mathrm{standard.} \mathrm{Data} \mathrm{points}$ are the mean values of three technical replicates, and error bars indicate \pm SEM.

Several different methods were trialled to improve the kinetic profile of indigoidine synthesis to make it more like a typical Michaelis-Menten curve. This included increasing the DMSO concentration in an attempt to improve the solubility of indigoidine during synthesis, changing the $\mathrm{pH}$ of the reaction medium, and using ascorbic acid to protect indigoidine from being reduced to the colourless leuco form (the role of ascorbic acid in modifying indigoidine is discussed in detail in Chapter Six). None of these methods gave a significant improvement on the optimised protocol described in Section 2.10.2. Due to the lower level of reproducibility and accuracy when using kinetic measurements, alternative measurement techniques were investigated.

\subsection{Development of a two-step resolubilisation protocol}

I considered that the conversion to the colourless leuco form might be one contributing factor to the characteristic absorbance profile of indigoidine production over time. However, visual examination of the wells after prolonged synthesis of indigoidine 
indicated the dominant factor was more likely that indigoidine was dropping out of solution, evidenced by formation of a slight blue precipitate. It has previously been shown that indigoidine is soluble in a limited range of organic solvents (including DMSO, THF, NMP, and DMF) (Novakova et al., 2010). DMSO was selected for use here, owing to its minimal toxicity and general availability in the laboratory setting.

To test whether indigoidine could be effectively resolublised in DMSO under our assay conditions, indigoidine was synthesised in $40 \mu \mathrm{L}$ replicates of reaction mix. Seven hundred and $60 \mu \mathrm{L}$ of various ratios of $\mathrm{ddH}_{2} \mathrm{O}$ and DMSO were then added to individual replicates to bring the total volume up to $800 \mu \mathrm{L}$, after which resolubilisation was attempted by shaking at $2,000 \mathrm{rev} / \mathrm{min}$ for $20 \mathrm{~min}$. I found that indigoidine became fully soluble at final concentrations of $80 \%$ DMSO (v/v) and above (Fig. 5.4A). Resolubilisation of indigoidine also showed that spontaneous conversion to a colourless leuco form was not substantially impairing my ability to accurately estimate L-glutamine concentrations within the timeframe of the assay. To test this, the absorbance of $200 \mu \mathrm{L}$ of solubilised indigoidine in 95\% DMSO, prepared as per Fig. 5.4A, was monitored at $590 \mathrm{~nm}$ for $10 \mathrm{~h}$ at $25^{\circ} \mathrm{C}$ (Fig. 5.4B). While the absorbance readings did decrease slowly over time, the rate of decrease was insufficient to interfere with accurate measurement of L-glutamine within the timeframe of the assay. 
A

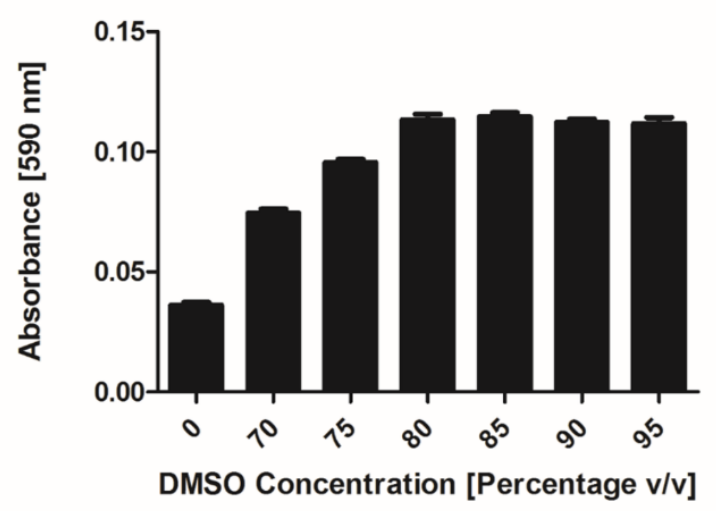

B

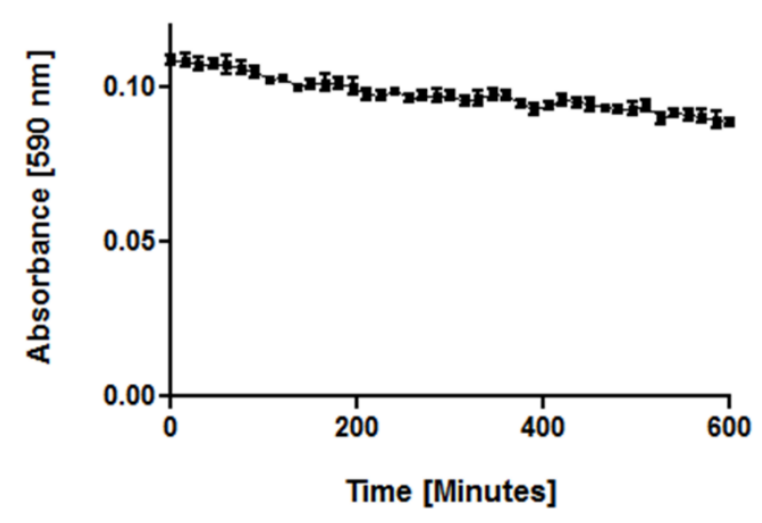

Figure 5.4: Resolubilisation of indigoidine in DMSO. (A). Replicate reaction mixes containing $50 \mathrm{mM}$ Tris- $\mathrm{Cl}, 20 \mathrm{mM}$ $\mathrm{MgCl}_{2}, 12 \mathrm{mM}$ ATP, $3 \mu \mathrm{M}$ holo-BpsA, $5 \mathrm{mM}$ L-glutamine and $\mathrm{ddH}_{2} \mathrm{O}$ with a total volume of $40 \mu \mathrm{L}$ were incubated for $1 \mathrm{~h}$ at $25{ }^{\circ} \mathrm{C}$. Addition of DMSO to final concentrations of $80 \%$ or higher were found to fully resolubilise the indigoidine present in an aqueous solution, enabling more accurate quantification via measurement of absorbance at $590 \mathrm{~nm}$. (B). The $A_{590}$ of a fully solubilised $200 \mu \mathrm{L}$ solution of indigoidine in $95 \%$ DMSO was found to diminish slightly over a tenhour period, consistent with indigoidine undergoing a gradual conversion into the colourless leuco isoform. Data points are the mean values from two independent experiments, each comprising three technical replicates and error bars indicate \pm SEM.

\subsubsection{Optimisation and miniaturisation of indigoidine solubilisation protocol}

To accurately quantify the amount of L-glutamine present in a sample using BpsA it is necessary to catalyse its complete conversion to indigoidine, followed by the complete solubilisation of the indigoidine formed. It was therefore essential to employ a reaction volume that would still allow DMSO to be added to a final concentration of at least $80 \%$ $(\mathrm{v} / \mathrm{v})$ prior to $\mathrm{A}_{590}$ measurement. Using $30 \mu \mathrm{L}$ of reaction mix and $10 \mu \mathrm{L}$ of sample was sufficient to reliably achieve this in a standard 96 well flat bottomed microplate with a well volume of $360 \mu \mathrm{L}$. After the conversion of L-glutamine to indigoidine was completed, $200 \mu \mathrm{L}$ of anhydrous DMSO was added to the original $40 \mu \mathrm{L}$ reaction volume, resulting in a final concentration of $83 \%$ DMSO. 
To identify a suitable reaction time to allow all of the L-glutamine in the reaction mix to be converted to indigoidine prior to addition of DMSO replicate $10 \mu \mathrm{L}$ samples of $1 \mathrm{mM}$ L-glutamine in $\mathrm{dd}_{2} \mathrm{O}$ were individually added to $30 \mu \mathrm{L}$ aliquots of reaction mix (comprising $50 \mathrm{mM}$ Tris-Cl pH 8.5, $10 \mathrm{mM} \mathrm{MgCl}$, $5 \mathrm{mM} \mathrm{ATP,} 3 \mu \mathrm{M}$ holo-BpsA). These were incubated at $25^{\circ} \mathrm{C}$ with shaking at $200 \mathrm{rev} / \mathrm{min}$. At $10 \mathrm{~min}$ intervals, groups of three replicates were stopped by the addition of $200 \mu \mathrm{L}$ DMSO. It was found that under these conditions complete conversion of L-glutamine to indigoidine had occurred within $50 \mathrm{~min}$ (Fig. 5.5A). It was also important to ensure complete solubilisation of the indigoidine, to enable accurate $A_{590}$ readings. To identify a suitable incubation time for resolubilisation of indigoidine in a final concentration of $83 \%$ DMSO $(v / v)$, replicate reaction mixes as detailed above were incubated at $25^{\circ} \mathrm{C}$ and $2,000 \mathrm{rev} / \mathrm{min}$, with $A_{590}$ readings taken every $5 \mathrm{~min}$. After $15 \mathrm{~min}$ the indigoidine was completely solubilised (Fig. $5.5 B)$.

A

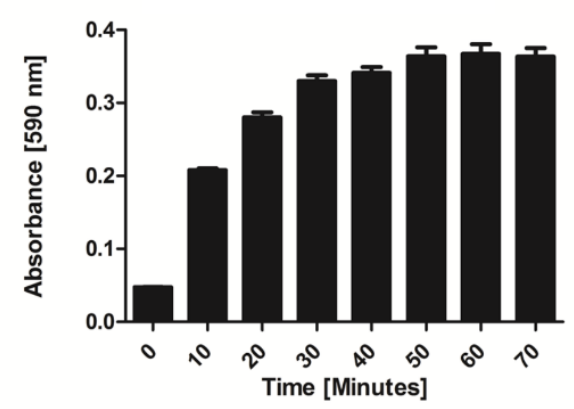

B

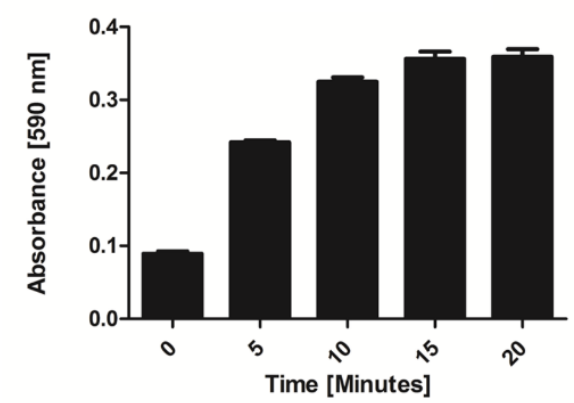

Figure 5.5. Optimisation of assay incubation steps. (A). For a reaction mix comprising $1000 \mu \mathrm{M} \mathrm{L-glutamine,} 50 \mathrm{mM}$ Tris- $\mathrm{Cl} \mathrm{pH} \mathrm{8.5,} 10 \mathrm{mM} \mathrm{MgCl}_{2}, 5 \mathrm{mM}$ ATP and $3 \mu \mathrm{M}$ holo-BpsA, increasing the reaction time to 50 min resulted in higher final $A_{590}$ values, indicating greater conversion of L-glutamine to indigoidine. Beyond $50 \mathrm{~min}$, no additional indigoidine production was measurable. Data are the mean values from two independent experiments, each comprising three technical replicates and error bars indicate \pm SEM. (B). Increasing the incubation time to 15 min post-addition of $83 \%$ $(\mathrm{v} / \mathrm{v})$ DMSO was found to increase the $A_{590}$ signal generated due to solubilisation of indigoidine. After $15 \mathrm{~min}$ no further indigoidine solubilisation was observed. Data are the mean values from two independent experiments, each comprising three technical replicates, and error bars indicate \pm SEM.

Using these optimised reaction conditions ( $1 \mathrm{~h}$ reaction incubation, with a $30 \mu \mathrm{L}$ reaction mix comprising $50 \mathrm{mM}$ Tris- $\mathrm{Cl}$ pH 8.5, $10 \mathrm{mM} \mathrm{MgCl}$, 5 mM ATP, $3 \mu \mathrm{M}$ holo-BpsA, followed by resolubilisation in $200 \mu \mathrm{L}$ DMSO and a further incubation with shaking at $2,000 \mathrm{rev} / \mathrm{min}$ for $20 \mathrm{~min}$ at $25^{\circ} \mathrm{C}$ ). To test the accuracy of the assay in measuring a 
range of L-glutamine concentrations in $10 \mu \mathrm{L}$ samples. Using standards of a $10 \mathrm{mM}$ stock solution of L-glutamine diluted to give a range of $0-1000 \mu \mathrm{M}$ in $\mathrm{ddH}_{2} \mathrm{O}$, highly reproducible linear standard curves with excellent $r^{2}$ values (e.g., 0.9992; Fig. 5.6A, B) could now be generated. Empirical testing revealed that the reaction was linear to approximately $2000 \mu \mathrm{M}$ L-glutamine, provided the incubation times for indigoidine formation and re-solubilisation were also increased (Fig 5.6D). A similar curve using $10 \mu \mathrm{L}$ standards of L-glutamine with a lower concentration range (0-100 $\mu \mathrm{M}$ in $\left.\mathrm{ddH}_{2} \mathrm{O}\right)$ had a lower $r^{2}$ value (0.9769; Fig. 5.6C), indicating greater variability within this range, albeit still a high level of accuracy. No difference in signal was observed between the $0 \mu \mathrm{M}$ and $20 \mu \mathrm{M}$ standards, indicating that $20 \mu \mathrm{M}$ L-glutamine is the detection limit of the assay using these parameters. 
A

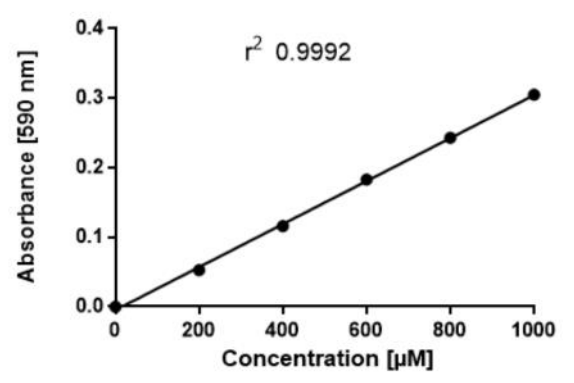

C

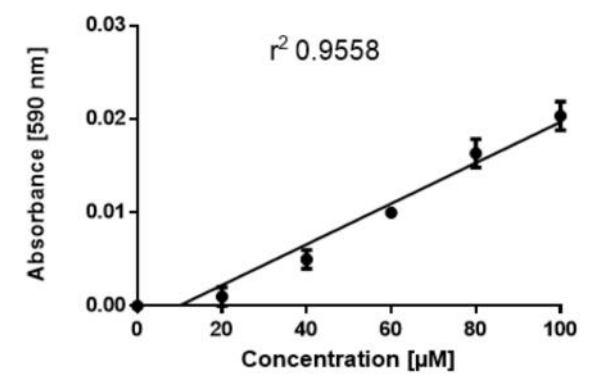

B

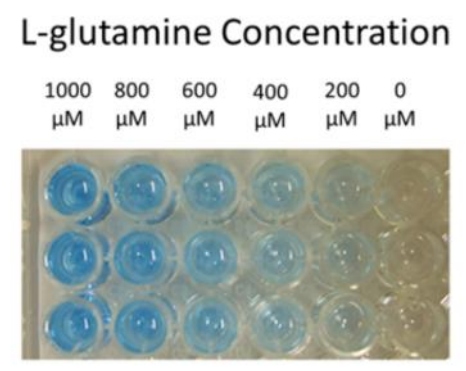

D

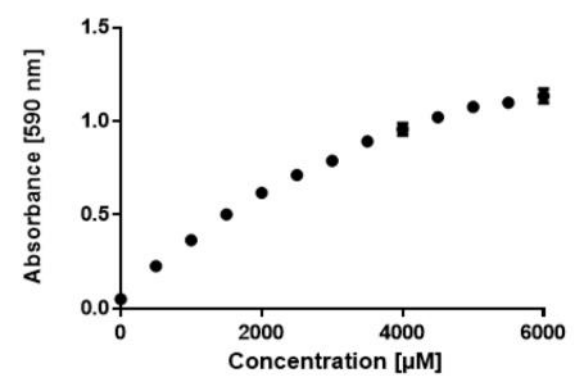

Figure 5.6 Generation of a standard curve using the optimised protocol: (A) A linear standard curve was generated by incubating $10 \mu \mathrm{L} \mathrm{L-glutamine} \mathrm{standards} \mathrm{at} \mathrm{a} \mathrm{range} \mathrm{of} \mathrm{concentrations} \mathrm{(} 0$ to $1000 \mu \mathrm{M}$ ) with $30 \mu \mathrm{L}$ of reaction mix (50 $\mathrm{mM}$ Tris-Cl pH 8.5, $10 \mathrm{mM} \mathrm{MgCl} 2,5 \mathrm{mM}$ ATP, $3 \mu \mathrm{M}$ holo-BpsA in $\mathrm{ddH}_{2} \mathrm{O}$ ) for $1 \mathrm{~h}$ at $25^{\circ} \mathrm{C}$. This was followed by resolubilisation in $200 \mu \mathrm{L}$ DMSO and a further incubation with shaking at $2000 \mathrm{rev} / \mathrm{min}$ for $20 \mathrm{~min}$ at $25^{\circ} \mathrm{C}$. Data are the mean of three technical replicates and error bars indicate \pm SEM. The data was normalised to zero for the $0 \mu \mathrm{M} \mathrm{L-}$ glutamine standard, and the $r^{2}$ value was calculated using Graphpad Prism. (B) An image of a standard curve showing the pigment intensity proportionate to the starting levels of L-glutamine present in each sample. Three replicates are shown. (C) A linear standard curve established as per panel A, only using $10 \mu \mathrm{L}$ of L-glutamine standards with a concentration range of $0-100 \mu \mathrm{M}$. No signal was detectable below $20 \mu \mathrm{M} \mathrm{L-glutamine.} \mathrm{(D)} \mathrm{Increasing} \mathrm{the}$ concentration of L-glutamine past $2000 \mu \mathrm{M}$ reduces the linear nature of the standard curve. The reactions were generated as per panel $\mathrm{A}$ with a concentration range between 0 and $1000 \mu \mathrm{M}$.

\subsection{Assay performance in common laboratory growth media}

Possible applications of a glutamine quantification assay include measuring levels of the essential but unstable additive L-glutamine in mammalian cell culture media, and measuring levels of L-glutamine yield from an industrial bacterial producer strain in bacterial culture media. To test whether our BpsA assay could accurately quantify Lglutamine levels in diverse culture media, samples of Lysogeny Broth (LB) and two mammalian cell culture media, DMEM (Dulbecco's Modified Eagle Medium) and MCDB (Molecular, Cellular, and Developmental Biology) medium were each spiked with $400 \mu \mathrm{M} \mathrm{L-glutamine}$ and compared against non-spiked controls. The values for the non- 
spiked media did not show any intrinsic variation when compared to water, indicating that there was no L-glutamine present in the un-amended media and that the colour of each medium did not fundamentally interfere with the detection of indigoidine (Fig.

5.7A). Importantly, the levels of indigoidine measured in each L-glutamine supplemented medium were reproducible and accorded with the $400 \mu \mathrm{M}$ spiking level (Table 5.1). LB was observed to have a slightly lower estimated level than the other media; this is potentially due to a component of LB either interfering with the synthesis of indigoidine or reducing indigoidine into the colourless leuco form. This phenomenon is investigated in more detail in Section 6.4.

A
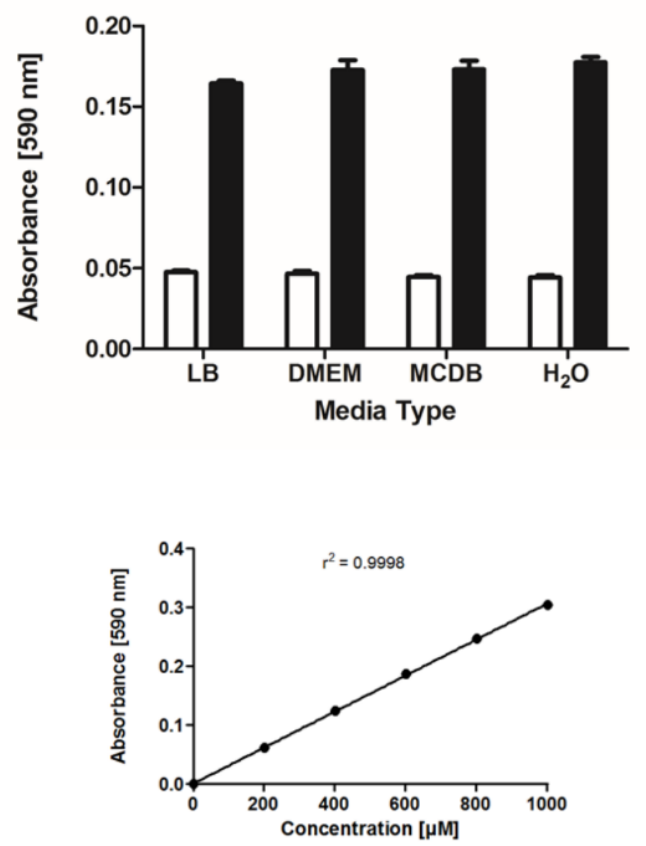

B
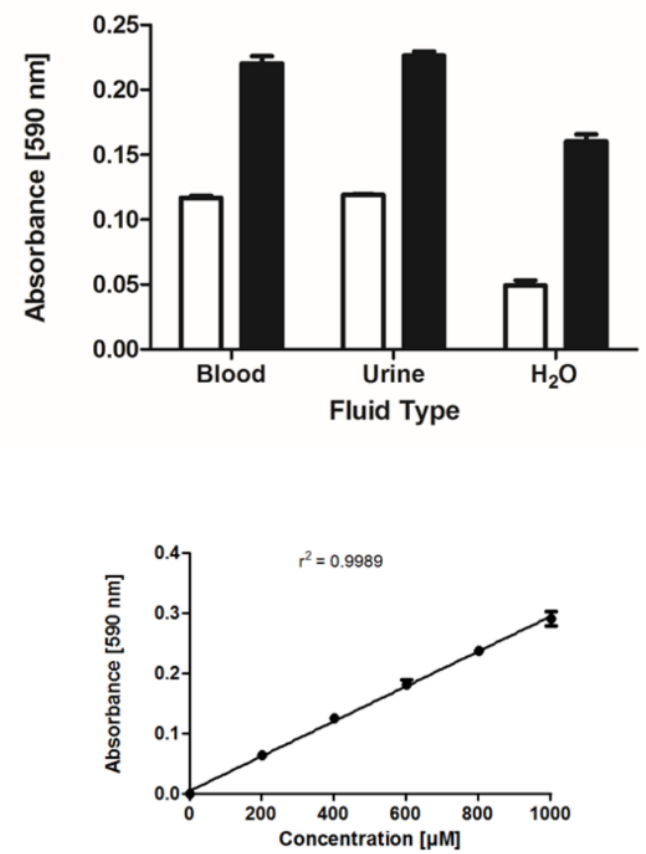

Figure 5.7 Measurement of L-glutamine concentrations in laboratory media and clinical relevant samples. (A) Spiked samples of L-glutamine are measurable in a range of common growth media. Thirty $\mu \mathrm{L}$ of reaction mix were added to each well. Test samples consisting of either $10 \mu \mathrm{L}$ un-amended media (white bars) or $10 \mu \mathrm{L}$ media to which had been added $400 \mu \mathrm{M} \mathrm{L-glutamine} \mathrm{(black} \mathrm{bars),} \mathrm{were} \mathrm{added} \mathrm{to} \mathrm{each} \mathrm{well.} \mathrm{The} \mathrm{reactions} \mathrm{were} \mathrm{then} \mathrm{incubated} \mathrm{for} 1 \mathrm{~h}$ at $25^{\circ} \mathrm{C}$ to fully convert the L-glutamine into indigoidine, after which samples were resolublised by addition of DMSO. Data are the means of three replicates and error bars indicate \pm SEM. (B) Biological fluids were assayed for Lglutamine, using spiked samples in the same manner as A. Test samples consisted of either urine or de-proteinated plasma, with $\mathrm{dd}_{2} \mathrm{O}$ added at a 1:1 ratio. The standard curves are displayed below Figure $A$ and Figure $B$ were used to calculate the values displayed in Table 5.1. The standard curves were prepared in the same manner as described in Fig. 5.6 The $\mathrm{r}^{2}$ values were calculated using Graphpad Prism. Data are the means of three replicates and error bars indicate \pm SEM. 


\subsection{Assay performance in clinically relevant biological fluids}

I next sought to determine whether the BpsA assay could also be used to measure Lglutamine concentrations in biological fluids such as blood plasma and urine. Although urine is known to vary in $\mathrm{pH}$ depending on factors such as fluid and food intake, it has previously been shown that holo BpsA is functional across a wide $\mathrm{pH}$ range (Owen et al., 2012), offering promise that the assay would be effective with urine samples (in contrast with the standard metabolite profiling technique of gas chromatography mass spectrometry, which is unreliable for quantification of L-glutamine in urine (Bouatra et al., 2013)). To test this, a fresh urine sample was first diluted $1: 1(\mathrm{v} / \mathrm{v})$ in $\mathrm{dd}_{2} \mathrm{O}$ (due to the reported concentrations of L-glutamine in blood plasma and urine being above $500 \mu \mathrm{M}$, which might cause the concentration of a spiked sample to be above $1 \mathrm{mM}$ (Preuss et al., 1966)). The diluted sample was then assayed in parallel with a sample that had been spiked with $400 \mu \mathrm{M}$ additional L-glutamine. The resulting $\mathrm{A}_{590}$ values showed a consistent difference between spiked and un-spiked samples (Fig. 5.7B, Table 5.1) indicating that the holo-BpsA was effective at measuring L-glutamine concentrations in urine.

In contrast, initial attempts to measure the concentration of L-glutamine in blood plasma failed. The addition of DMSO to the reaction mix containing $10 \mu \mathrm{L}$ of blood plasma sample caused the mix to become cloudy and prevented accurate $A_{590}$ readings from being obtained. I hypothesised this was due to the DMSO reacting with the proteins and cell debris present in the blood plasma, on the basis of empirical tests that revealed indigoidine could readily be generated and measured in commercially sourced adult bovine serum (data not shown). To remove these confounding constituents, the blood plasma was pre-treated by passing it through a column with $3 \mathrm{kDa}$ retention cutoff. Following this step, it was now possible to accurately measure the L-glutamine present in the serum. Samples consisting of a 1:1 (v/v) mixture of $d_{d H_{2}} \mathrm{O}$ and deproteinated blood were assayed and directly compared to replicate samples that had been spiked with an additional $400 \mu \mathrm{M}$ of L-glutamine. The resulting absorbance values clearly showed an increase in absorbance with the spiked blood plasma sample, corresponding to the predicted $400 \mu \mathrm{M}$ increase in L-glutamine content (Fig. 5.7B, Table 5.1). 


\begin{tabular}{|c|c|c|}
\hline Sample & Calculated value ( $\mu \mathrm{M})$ & A590 value \\
\hline LB + $400 \mu \mathrm{M}$ L-glutamine & $382.7 \pm 6.7$ & $0.164 \pm 0.002$ \\
\hline $\begin{array}{l}\text { DMEM + } 400 \mu \mathrm{M} \mathrm{L-} \\
\text { glutamine }\end{array}$ & $410.9 \pm 14.1$ & $0.173 \pm 0.006$ \\
\hline $\begin{array}{l}\text { MCDB + } 400 \mu \mathrm{M} \text { L- } \\
\text { glutamine }\end{array}$ & $411.6 \pm 12.5$ & $0.173 \pm 0.001$ \\
\hline $\mathrm{H}_{2} \mathrm{O}+400 \mu \mathrm{M}$ L-glutamine & $420.4 \pm 7.3$ & $0.181 \pm 0.003$ \\
\hline Blood plasma & $240.1 \pm 6.0$ & $0.117 \pm 0.002$ \\
\hline $\begin{array}{c}\text { Blood plasma }+400 \mu \mathrm{M} \text { L- } \\
\text { glutamine }\end{array}$ & $610.3 \pm 20.3$ & $0.220 \pm 0.006$ \\
\hline Urine & $248.4 \pm 2.1$ & $0.119 \pm 0.001$ \\
\hline $\begin{array}{l}\text { Urine }+400 \mu \mathrm{M} \text { L- } \\
\text { glutamine }\end{array}$ & $631.7 \pm 11.4$ & $0.226 \pm 0.003$ \\
\hline $\mathrm{H}_{2} \mathrm{O}$ & $-0.4 \pm 13.7$ & $0.049 \pm 0.004$ \\
\hline $\mathrm{H}_{2} \mathrm{O}+400 \mu \mathrm{M}$ L-glutamine & $396.0 \pm 19.1$ & $0.160 \pm 0.005$ \\
\hline
\end{tabular}

Table 5.1. Measurement of L-glutamine in spiked laboratory media and biological samples: Data is the mean of three replicates, with error bars \pm SD.

It is possible that the sample background might exert subtle effects on assay variability, as although our measurements of L-glutamine in the different culture media and biological fluids were consistent with the spiked levels, the errors associated with these measurements were typically higher than for the L-glutamine standards in $\mathrm{dd}_{2} \mathrm{O}$ (e.g., Fig. 5.7A). Nevertheless, our results show that the BpsA assay is generally robust for use in these different applications.

\subsection{Comparison with commercially available kits}

Current commercially available enzymatic based methods for the measurement of Lglutamine are generally based on a combination of the enzymes glutaminase and glutamate dehydrogenase, and require two consecutive deamination reactions to take place. Not only do these methods add an additional reaction step, it also importantly means that if a sample might contain both glutamine and glutamate then both "before" 
and "after" measurements need to be taken. The additional conversion and measurement steps add complexity, additional reagents and longer processing times.

For example, the GLN1 kit marketed by Sigma Aldrich ${ }^{\circledR}$ requires eight different reagents and multiple incubation steps, and moreover is difficult to miniaturise and rather expensive. A direct comparison between the two kits also shows that both kits are able to generate standard curves with similar levels of accuracy $\left(r^{2}=0.9991\right.$ for the Sigma Aldrich kit vs $r^{2}=0.9994$ for the glutamine biosensor). Our method only requires one enzyme, a buffering solution containing $\mathrm{MgCl}_{2}$, Tris- $\mathrm{Cl}$ and ATP, and DMSO as a stop solution. The requirement for only a single reaction to convert L-glutamine into indigoidine, followed by a simple re-solubilisation step, also improves assay speed. Moreover, should greater assay speed be required, the incubation times reported here can be greatly reduced by increasing the amount of holo-BpsA enzyme added to each reaction. Finally, the high-level stability of the enzyme is amenable to storage in a "kit" format, and the linear range of our assay $(20-1500 \mu \mathrm{M})$ is well suited for direct measurement of L-glutamine concentrations in blood which is reported to be between $700 \mu \mathrm{M}$ and $900 \mu \mathrm{M}$ (Lee et al., 2016; Trinh et al., 2003) and urine (Bouatra et al., 2013). However, some cell culture media might have to be diluted 2-4 fold prior to measurement to enable their initial L-glutamine concentrations to fall within this range (Vriezen et al., 1997).

\subsection{Alternative methods for the detection of L-glutamine}

In parallel, I considered whether conversion of indigoidine to its leuco form using a protocol previously established by Müller et al (2012) might provide an alternative means of accurately measuring L-glutamine concentrations. To test this, I first generated an indigoidine standard curve as for Fig. 5.6A, using $10 \mu \mathrm{L}$ samples of L-glutamine standards from 0-1000 $\mu \mathrm{M}$ (Fig. 5.8A). Two point three $\mu \mathrm{L}$ of the reducing agent sodium dithionite ( $0.15 \mathrm{~g}$ of sodium dithionate in $10 \mathrm{ml}$ of $1 \mathrm{M} \mathrm{NaOH}$ ) (Müller et al., 2012) was then added to each well, resulting in a complete conversion to the colourless leuco form. Subsequently, a standard curve was generated by monitoring the fluorescence of each well (ex $415 \mathrm{~nm} /$ em $520 \mathrm{~nm}$; Fig. 5.8B). The $\mathrm{r}^{2}$ value for the leuco fluorescence standard curve (0.4597) was far lower than the colorimetric indigoidine standard curve (0.9938), 
indicating that the former was a less accurate method for quantifying L-glutamine. At much higher concentrations of indigoidine it is possible that this method may be more useful. However, at the concentrations often found in physiological samples it is much more accurate to measure the indigoidine at $590 \mathrm{~nm}$ in the non-leuco form.

A

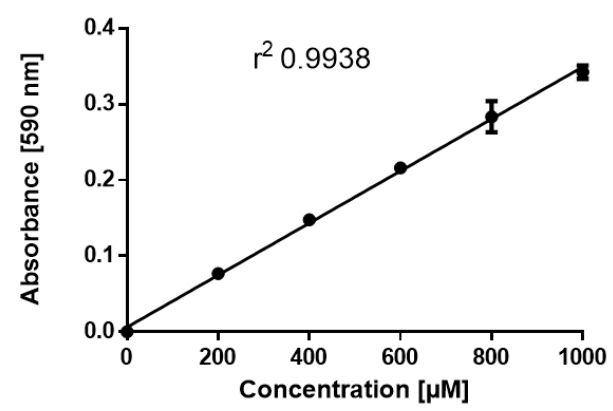

B

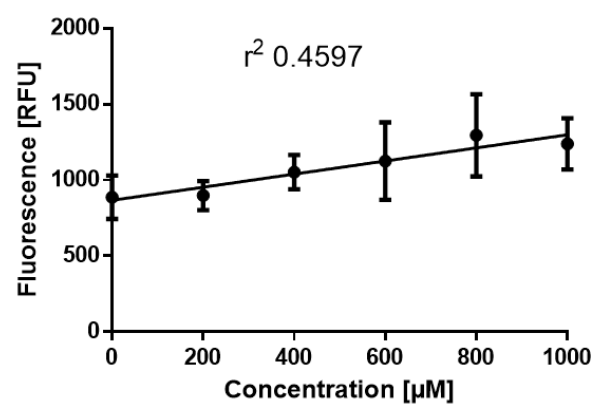

Figure 5.8 Converting indigoidine to its colourless leuco form does not provide a more accurate means of measuring L-glutamine. (A) A linear standard curve was generated by incubating $10 \mu \mathrm{L}$ of L-glutamine standards across a range of concentrations $(0-1000 \mu \mathrm{M})$ with $30 \mu \mathrm{L}$ of a reaction mix Data are the means of three replicates and error bars indicate \pm SEM. (B) After the standard curve was recorded, $2.3 \mu \mathrm{L}$ of a reducing agent $(0.15 \mathrm{~g}$ of sodium dithionate in $10 \mathrm{ml}$ of $1 \mathrm{M} \mathrm{NaOH}$ ) was added to each well to convert all indigoidine into the colourless leuco form. Following $2 \mathrm{~min}$ incubation at 2,000 rev/min, fluorescence measurements (ex $415 \mathrm{~nm} / \mathrm{em} 520 \mathrm{~nm}$ ) were used to generate a standard curve. The low $\mathrm{r}^{2}$ value indicates that this is not a reliable means of measuring L-glutamine. Data are the means of three replicates and error bars indicate \pm SEM.

\subsection{Long term stability of BpsA}

For BpsA to be broadly useful it is necessary that the enzyme retain stability and activity for extended periods of time, so that it need not be purified anew prior to each assay. To evaluate the shelf life of the enzyme, a preparation of holo-BpsA was generated and its maximal rate of indigoidine synthesis measured as $(7.48 \pm 0.03) \times 10^{-4} \Delta \mathrm{A}_{590} \mathrm{~s}^{-1}$ in triplicate assays using a master mix that included $1000 \mu \mathrm{M} \mathrm{L-glutamine.} \mathrm{The} \mathrm{holo-BpsA}$ was then stored at $-20^{\circ} \mathrm{C}$ in storage buffer $(50 \mathrm{mM}$ sodium phosphate buffer $\mathrm{pH} 7.8$ containing $40 \%(\mathrm{v} / \mathrm{v})$ glycerol). When this activity assay was repeated 11 months later, the mean maximal rate of indigoidine synthesis was measured as $7.18 \pm 0.17 \times 10^{-4} \Delta \mathrm{A}_{590}$ $\mathrm{s}^{-1}$, indicating that BpsA retains $>95 \%$ activity during long term storage at $-20^{\circ} \mathrm{C}$ (Fig. $5.9 A)$. 
It would also be beneficial if the enzyme were at least moderately stable at $4{ }^{\circ} \mathrm{C}$ and $25^{\circ} \mathrm{C}$, so that it does not lose activity during routine handling. To test this, BpsA was prepared and stored (in storage buffer, as above) in the apo form and was converted into the holo-form immediately prior to kinetic measurements. Following storage for 24 weeks at $4{ }^{\circ} \mathrm{C}$ it was found that nearly full activity was retained $(95.7 \pm 1.8 \%)$ compared to the sample stored at $-20^{\circ} \mathrm{C}$ (Fig. 5.9B). In contrast, the sample stored at $25^{\circ} \mathrm{C}$ had retained only $9.1 \pm 1.9 \%$ of the starting level of activity after 24 weeks.

Collectively, these data indicate that BpsA is stable for at least 11 months and its activity is unlikely to be significantly impaired by routine handling. The enzyme can be stored effectively in either a freezer or refrigerator, however the optimal storage temperature is $-20^{\circ} \mathrm{C}$.

A

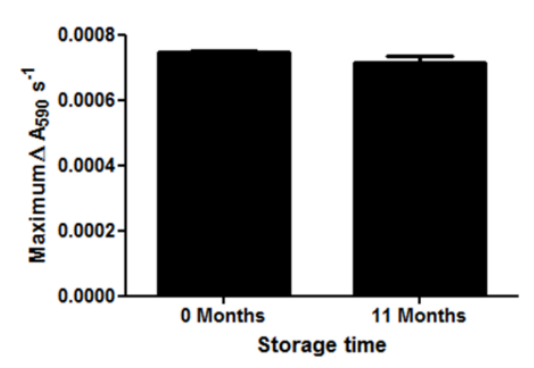

B

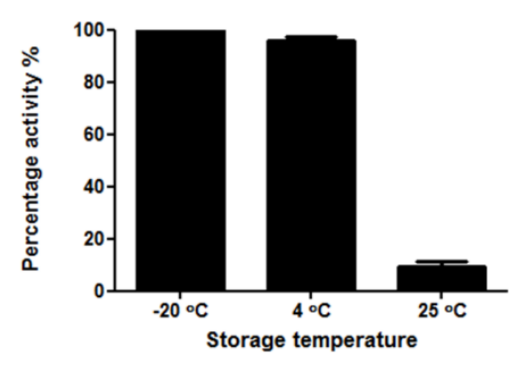

Figure 5.9 Effect of long term storage on BpsA activity. (A) Holo-BpsA was assayed for activity (maximal reaction velocity) before and after storage at $-20^{\circ} \mathrm{C}$ for 11 months. In each case a master mix containing $2 \mu \mathrm{M}$ holo-BpsA, 50 $\mathrm{mM}$ Tris- $\mathrm{Cl} \mathrm{pH} \mathrm{8.5,} 20 \mathrm{mM} \mathrm{MgCl}, 5 \mathrm{mM}$ ATP and ddH2O to a final volume of $90 \mu \mathrm{L}$ was dispensed into individual wells of a 96 well plate. The reaction was initiated by the addition of $10 \mu \mathrm{L} 1000 \mu \mathrm{M} \mathrm{L}$-glutamine in ddH2O. The 96 well plate was shaken at $1000 \mathrm{rev} / \mathrm{min}$ for $10 \mathrm{~s}$ and the $A_{590}$ values were recorded every $10 \mathrm{~s}$ for $1 \mathrm{~h}$. The maximal velocity of the reaction was calculated by finding the maximum slope value as previously described. Data are the means of three replicates and error bars indicate \pm SEM. (B) BpsA was assayed for activity (maximal reaction velocity) before and after storage at either $-20^{\circ} \mathrm{C}, 4^{\circ} \mathrm{C}$ or $25^{\circ} \mathrm{C}$ for 24 weeks. To convert the apo-BpsA to holo-BpsA prior to activity assays the method in Section 2.7.1 was used. Indigoidine synthesis was initiated by the addition of $25 \mu \mathrm{L}$ of $5 \mathrm{mM}$ ATP and $2 \mathrm{mM} \mathrm{L-glutamine} \mathrm{in} \mathrm{ddH}_{2} \mathrm{O}$ (concentrations are per final $100 \mu \mathrm{L}$ reaction volume). The 96 well plate was then incubated at $25^{\circ} \mathrm{C}$ with shaking at $1000 \mathrm{rev} / \mathrm{min}$ for $10 \mathrm{~s}$ and the A590 values were recorded every $10 \mathrm{~s}$ for $1 \mathrm{~h}$. The velocity of the reaction was calculated by finding the maximum slope value as above, and percentage activity was calculated for each sample relative to the pre-storage level of activity. The graph bars are the mean of three replicates and the error bars indicate \pm SEM.

\subsection{Chapter discussion}

This chapter outlines the development of a novel method to detect L-glutamine in a range of biologically and clinically relevant conditions. BpsA is well suited for use in quantitative in vitro assays. Compared to other NRPSs, it is a small and simple enzyme. Following its conversion to the holo form by a PPTase partner, it is fully autonomous in 
its ability to generate a pigmented product from L-glutamine and ATP. Also, unusual for an NRPS enzyme, it is easily purified in a soluble form via common purification methods such as nickel affinity chromatography. Other important advantages of BpsA in this context include its tolerance to a relatively wide $\mathrm{pH}$ range, its sensitivity and ability to function in diverse media compositions, and its lengthy shelf life during refrigerated storage. One minor limitation however is that due to the mild antimicrobial properties of indigoidine it is difficult to express BpsA in E. coli in its active holo form. To circumvent this, I instead expressed BpsA in the inactive apo form and added the Ppant prosthetic group during the purification process. By mixing the lysate of an E. coli culture expressing $6 \mathrm{His}$ tagged apo-BpsA with that from an E. coli culture expressing non-tagged PcpS, large amounts of holo-BpsA could be quickly generated in a in a cost-effective manner. Mixing crude lysates also allowed us to take advantage of the coenzyme $A$ naturally present in those lysates, likely reducing the amount of exogenous coenzyme $A$ required to be added during the activation process.

Initial attempts to develop BpsA as a biosensor were frustrated by the insolubility of indigoidine in aqueous media, the characteristic rapid rise and then drop in $A_{590}$ values during synthesis making it difficult to obtain accurate measurements. While there was some correspondence between the glutamine concentration present in a starting sample and the maximal rate of indigoidine synthesis or the initial maximum $A_{590}$ value reached, it was not possible to consistently generate a reliable linear standard curve from either of these parameters. The addition of a DMSO solubilisation step not only resolved these issues, but also facilitated miniaturisation of the assay, and meant a single end-point reading could be taken rather than requiring continuous monitoring of the reaction. It is also possible that the mild oxidant properties of DMSO had the added effect of inhibiting conversion to the colourless leuco form of indigoidine, enhancing the robustness of our assay.

One exceptional scenario was encountered when attempting to measure the glutamine concentration in blood plasma, where the addition of DMSO caused the solution to become opaque, interfering with $A_{590}$ measurement in a standard plate reader. To test whether the opaque effect was being caused by DMSO-mediated aggregation of proteins and other cell debris present in the blood plasma, the red blood cells were 
removed by centrifugation then purified the supernatant by passing it through a $3 \mathrm{kDa}$ cut-off column. These processing steps caused the blood plasma to turn from a pale orange/pink colour to a clear fluid, which was then amenable to BpsA-mediated glutamine quantification.

In addition to the L-glutamine assay described here, BpsA has previously been used as a biosensor in several applications including in bacterial species (Müller et al., 2012). It might also have utility as a reporter for strains of bacteria that are used to produce commercial quantities of L-glutamine in an industrial setting, possibly even enabling directed strain improvement experiments to increase output. Our discovery that DMSO may be added to increase the accuracy of the measurements taken may improve assay accuracy and sensitivity in many of these other applications. 


\section{Chapter 6-Additional applications of BpsA}

\subsection{Manuscript in preparation}

Optimisation of the NRPS BpsA in a single-enzyme colorimetric assay for quantification of ATP and its application in evaluating the substrate specificity of other NRPS adenylation domains

Alistair S. Brown, Mark J. Calcott and David F. Ackerley

\subsection{Summary}

This chapter describes additional applications of $\mathrm{BpsA}$, beginning with development of a biosensor to detect and quantify ATP using a variation of the BpsA method presented in Chapter Five. As with L-glutamine, BpsA requires two molecules of ATP to synthesise one molecule of indigoidine, thus if ATP is limiting there will be a direct correlation between the amount of ATP in a sample and the quantity of indigoidine synthesised. The BpsA biosensor was optimised for the detection of ATP and then used to quantify the concentration of ATP in a variety of samples including blood and cell culture media.

In addition, the performance of the biosensor in LB was analysed and methods were explored to further refine the assay. These included assessing the effect of establishing standard curves in the media being tested, and use of the antioxidant L-ascorbic acid to try and stabilise indigoidine. Proof of concept studies and assay optimisation were undertaken to investigate whether this new ATP assay might have utility in evaluating the substrate specificity of purified NRPS A domains.

The ability of BpsA to convert the important glutamine analogue L-Alanyl-L-Glutamine into an indigoidine-like compound was also investigated. The compound synthesised from alanyl-glutamine had different solubility properties and absorbed at a higher wavelength than indigoidine. Analysis using HPLC showed that it had different retention times when compared to indigoidine, providing strong evidence that a new compound 
was being synthesised. Finally, some initial exploratory work was undertaken to determine if an enzyme present in an indigoidine producing gene cluster was responsible for the conversion of indigoidine to the colourless leuco form.

\subsection{Introduction}

\subsubsection{Detection and quantification of ATP}

ATP is a vital fuel source for cells that is responsible for driving numerous biological reactions that are crucial for life. In addition, it is an important signalling molecule (Taylor et al., 1998). Due to its ubiquitous nature in biological systems it is important to be able to accurately quantify ATP concentrations in complex biological conditions. Detecting ATP has a wide range of uses including as a surrogate for measuring bacterial contamination of products and surfaces (Costa et al., 2006), measuring the viability of human cells (Petty et al., 1995), or measuring the activity of enzymes (Lundin, 2000). Several different assays that can accurate quantify ATP have been developed.

The most sensitive method of ATP quantification uses the enzyme firefly luciferase. Firefly luciferase converts luciferin in a two-step oxidation reaction to oxylucifernin, which results in the emission of light at $560 \mathrm{~nm}$. The emission of light is directly proportional to the starting concentration of ATP in a sample (Lundin et al., 1976). The luciferase assay was first developed in the 1970s and allows the detection of ATP down to a picomolar range (Kimmich et al., 1975). Since then, improvements to both the assay and detection equipment has made it possible to measure ATP in the attomolar range (Okanojo et al., 2017). This level of sensitivity means that the detection of ATP can potentially be used to identify single bacterial cells.

Despite the accuracy and sensitivity of the firefly luciferase system, many laboratories do not have access to luminometers. In order to fill this gap in the market, several different colourimetric methods have been developed. These assays lack the sensitivity of the firefly luciferase system, but are compatible with common laboratory spectrophotometers. The most common of these assays is based on the phosphorylation of glycerol by glycerol kinase. The glycerol-3-phosphate is then converted in subsequent reactions to a coloured product that is detectable at $570 \mathrm{~nm}$. 
Kits relying on this method are available from a range of suppliers, including SigmaAldrich, Genway Biotech, and ApexBio Technology. These kits report having a detection limit of approximately $1 \mu \mathrm{M}$. While this reaction is an effective method to determine the concentrations of ATP it does have a few flaws, in that multiple steps are required to produce a coloured product, and it relies on the test sample not containing any glycerol3-phosphate. Other assays that have been reported for the detection of ATP have used aptamers (Liu et al., 2011; Zeng et al., 2012) or a range of different compounds (Sancenón et al., 2001), however to the best of my knowledge none of these other assays are commercially available.

In an attempt to address the limitations of the glycerol kinase kit and to expand the usefulness of the BpsA biosensor, I tested the ability of BpsA to accurately measure ATP. By replacing L-glutamine with ATP as the concentration-limiting co-substrate I reasoned that the amount of indigoidine synthesised would be directly proportional to the starting concentration of ATP in a sample (Fig. 6.1). I also sought to test whether this method might have utility in monitoring the adenylation reaction catalysed by A domains by following the consumption of ATP during the reaction.

\section{Excess of}

Holo-BpsA

Indigoidine

\section{L-glutamine}<smiles>NC(=O)CCC(N)C(=O)O</smiles>

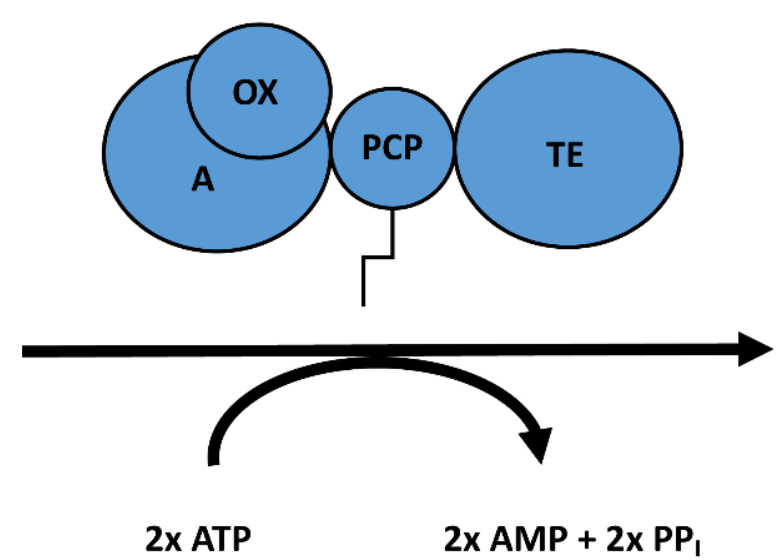<smiles>NC(=O)CCC(N)C(=O)O</smiles>

2x ATP

$2 \times A M P+2 x P P_{1}$

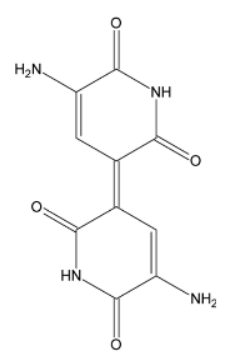

Figure 6.1 Schematic diagram illustrating the synthesis of indigoidine by BpsA: Two molecules of L-glutamine are converted into the blue pigment indigoidine by the NRPS BpsA. The synthesis of indigoidine is an ATP dependant reaction. Thus, for every one molecule of indigoidine produced, two molecules of ATP are consumed. 


\subsubsection{Detection of adenylation by A domains}

Another use for the ATP biosensor is the detection of adenylation reactions by monitoring the consumption of ATP. For NRPS modules of unknown monomer substrate specificity it is useful to be able to detect if adenylation is occurring in order to define the specificity of $A$ domains. Being able to experimentally determine the specificity of an A domain is important; although prediction software based on the Stachelhaus code (Stachelhaus et al., 1999) can provide some information towards the specificity of an A domain it is far from definitive, and is particularly inaccurate for eukaryotic NRPSs or A domains that accept unusual substrates (Lee et al., 2010). As such, it is essential to be able to experimentally confirm the substrate specificity of previously uncharacterised $A$ domains.

A domains catalyse the conversion of ATP and an amino acid to aminoacyl adenylate and pyrophosphate (PPi) in an equilibrium reaction (Mootz and Marahiel, 1997). However, detecting adenylation is not always simple, and a range of different assays have been developed to quantify the amount of PPi released. One of the more commonly used assays is the PPi-ATP exchange assay (Otten et al., 2007). This assay works by adding an excess of PPi, a proportion of which is radiolabelled ( $\left.{ }^{32} \mathrm{PPi}\right)$, which shifts the reversible equilibrium to the formation of radiolabelled ATP, which is then absorbed by activated charcoal. ATP can then be eluted from the charcoal in an aqueous medium and the extent of radiolabelling can then be measured and is directly proportional to the level of activity the A domain has with a particular amino acid substrate (Otten et al., 2007).

Another common method to measure adenylation is the malachite green assay, a colourimetric assay (McQuade et al., 2009). The malachite green assay uses pyrophosphatase to cleave the PPi resulting from ATP hydrolysis into two molecules of phosphate, which then form a complex with malachite green and molybdate, causing a colour change. Since the amount of PPi released is proportional to the level of adenylation occurring it is possible to quantify activity with a candidate substrate by measuring the formation of coloured complex (McQuade et al., 2009).

Both of these assays, despite their common usage, do have some drawbacks. The PPiATP exchange assay requires radioactive material and specialised equipment to detect 
the amount of radiation being produced. While the assay has been adapted for 96 well plate format it is still quite labour intensive (McQuade et al., 2009). The malachite green assay requires inexpensive reagents and it has been adapted for use in both 384 well plates and 96 well plates. However, the product formed is not always stable and it is sensitive to contaminating phosphate species (McQuade et al., 2009).

\subsection{Development of an assay to detect and quantify ATP}

I first sought to detect whether BpsA could be used to generate a linear standard curve across a range of ATP concentrations in $\mathrm{dd}_{2} \mathrm{O}$, and to confirm that there was a 1:1 stoichiometry between the amount of ATP consumed and the amount L-glutamine consumed during indigoidine synthesis. A possible reason for this not being the case might be that the adenylation reaction of BpsA (an equilibrium reaction) could occasionally be futile, resulting in the consumption of ATP without the adenylated Lglutamine being converted into indigoidine.

To confirm that a linear standard curve could be established for a range of ATP concentrations the following protocol optimised in chapter five and described fully in Section 2.10.6 was used; $10 \mu \mathrm{L}$ ATP standards in $\mathrm{dd}_{2} \mathrm{O}$ with a range of concentrations from 0 to $1000 \mu \mathrm{M}$ were incubated along with $30 \mu \mathrm{L}$ of reaction mix (50 mM Tris-Cl pH 8.5, $10 \mathrm{mM} \mathrm{MgCl} 2,5 \mathrm{mM} \mathrm{L-glutamine,} 3 \mu \mathrm{M}$ holo-BpsA in $\mathrm{ddH}_{2} \mathrm{O}$ ) for $1 \mathrm{~h}$ at $25^{\circ} \mathrm{C} 200$ $\mathrm{rev} / \mathrm{min}$ in a 96 well plate. The synthesised indigoidine was then resolublised by the addition of $200 \mu \mathrm{L}$ of DMSO with the 96 well plate being mixed at $2000 \mathrm{rev} / \mathrm{min}$ for 15 $\min$. The absorbance was measured at $590 \mathrm{~nm}$ and a standard curve generated using GraphPad Prism ${ }^{\circledR}$ as illustrated in Fig. 6.2A. The standard curve in this figure had an excellent $r^{2}$ value (0.9939), which was typical for the method and confirmed that a linear standard curve could be generated up to $1 \mathrm{mM}$ ATP.

To determine the minimum detection level for ATP, a standard curve with a concentration range of 0 to $100 \mu \mathrm{M}$ was established (Fig. 6.2B) using the protocol described in Section 2.10.6. The concentration of L-glutamine were the A590 value is significantly different from $0 \mu \mathrm{M}$ is $40 \mu \mathrm{M}(\mathrm{P}=0.0047)$, which is similar to that of $\mathrm{L}-$ glutamine. The standard curve for L-glutamine at a range of $0-100 \mu \mathrm{M}$ (Fig. 5.6C) had 
slightly lower $A_{590}$ values compared to the ATP curve, which could be caused by slight differences in the actual concentration of each standard. This could be due to ATP being purified as a salt (disodium trihydrate) resulting in inaccurate reported masses, or slight inaccuracies in the measurement of each standard.

One benefit of our assay over other colourimetric assays is the comparatively rapid nature of the assay. By increasing the concentration of BpsA the incubation time needed for accurate L-glutamine measurement could be reduced (Section 5.6.1). I found that increasing the concentration of BpsA to $12 \mu \mathrm{M}$ reduced the incubation time to 15 min for a $10 \mu \mathrm{L}$ standard of ATP at a concentration of $1500 \mu \mathrm{M}$ (Fig 6.2C). The assay was set up as described in Section 2.10.6 and the synthesis of indigoidine was stopped at each time point by the addition of $200 \mu \mathrm{L}$ of DMSO to the reaction mix. After $1 \mathrm{~h}$, the 96 well plate was shaken at $2000 \mathrm{rev} / \mathrm{min}$ and the $A_{590}$ values recorded. The reduction in incubation time resulted in a potential trade-off between time and cost due to the substantially higher concentrations of BpsA needed for the reaction to consume all of the ATP in the shorter incubation period. Due to the increased amount of BpsA required, the remainder of the assays described in this section were performed with the standard $3 \mu \mathrm{M}$ concentration. If reducing the assay running time is essential, increasing the concentration of BpsA is a viable option to achieve this. 
A

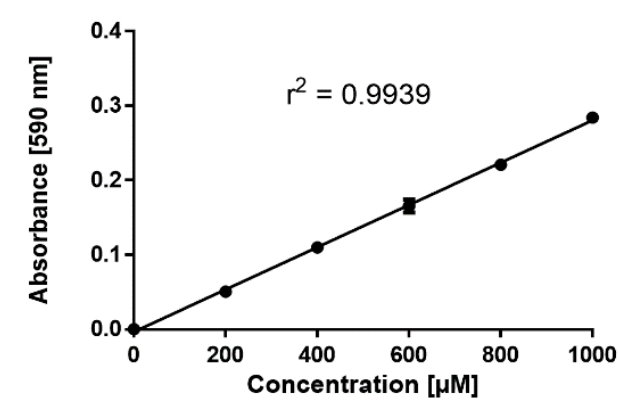

C

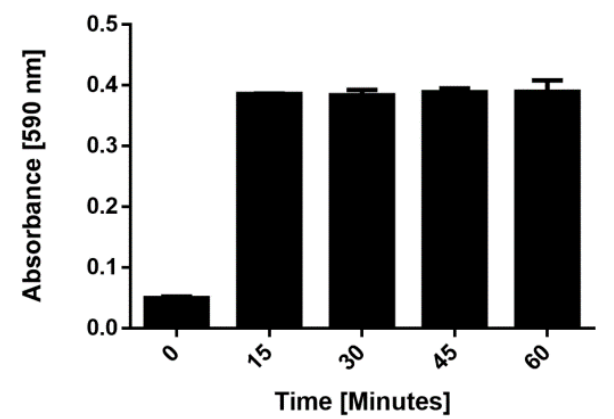

B

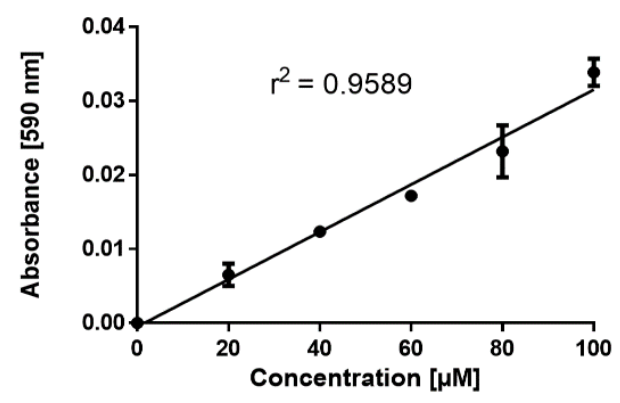

Figure 6.2 Optimisation and determination of reaction parameters for the ATP assay: (A) A standard curve was established using the optimised protocol described in Section 2.10.2. Graphpad Prism ${ }^{\circledast}$ was used to calculate the $r^{2}$ value. Each data point represents the average of three replicates with the error bars representing \pm SEM. (B) The minimum detection range of the assay is between 20 and $40 \mu \mathrm{M}$. Each data point represents the average of two biological replicates comprising of three technical replicates. Error bars represent \pm SEM. (C) The $A_{590}$ value of the reaction samples stopped every $15 \mathrm{~min}$ by the addition of $200 \mu \mathrm{L}$ of DMSO was measured. Each data point represents the average of three replicates with the error bars representing \pm SEM.

\subsection{Quantification of ATP levels in different cell culture media}

While the assay can quantify ATP accurately in $\mathrm{dd}_{2} \mathrm{O}$ it is unlikely that samples would always be present in such a simple vehicle. To determine if the assay could function effectively in a variety of different conditions, ATP concentrations were quantified in the laboratory media Lysogeny Broth (LB), used for the culturing of a range of bacterial species, and Roswell Park Memorial Institute Medium (RPMI), a common mammalian cell culture media. 


\subsubsection{Detection of ATP in LB}

A standard curve using ATP standards in $\mathrm{dd}_{2} \mathrm{O}$ with a concentration range from 0 to $1000 \mu \mathrm{M}$ was established using the optimised protocol as described in Section 6.3. The standard curve was then used to estimate the concentration of LB and RPMI supplemented with ATP at a final concentration of $500 \mu \mathrm{M}$. The predicted mean of the concentrations of ATP in LB was $423 \pm 34 \mu \mathrm{M}$ which was $\sim 15 \%$ lower than anticipated over two biological replicates each containing three technical replicates (errors represent +/- the SD).

This could be due to a compound or compounds within the sample degrading the ATP or indigoidine, or inhibiting BpsA, causing a reduction in the $A_{590}$ value and thereby resulting in a lower estimated concentration. To try and improve the accuracy of the assay in LB and to pinpoint the reason for the underestimation of the concentration of ATP in this medium, a series of experiments were performed to probe the effects of LB on indigoidine synthesis.

\subsubsection{Establishment of standard curves using the media being tested as a vehicle}

If a compound in the LB was interfering with indigoidine, for example reducing it to the colourless leuco form, this effect should be more pronounced at lower concentrations resulting in a less linear standard curve. To test if the media had an effect on the linearity of the standard curves an assay was set up as described in methods Section 2.10.6 except the media being tested was used as a vehicle for the ATP standards rather than $\mathrm{ddH}_{2} \mathrm{O}$. The standard curves are graphed in Fig. 6.3. When LB was compared to RPMI and $d \mathrm{dH}_{2} \mathrm{O}$, at each concentration of ATP the $\mathrm{A}_{590}$ for the LB sample was slightly lower. The $r^{2}$ value of 0.9875 for LB was also lower than the RPMI $r^{2}$ value of 0.9987 , which was similar to the $r^{2}$ value to the $\mathrm{ddH}_{2} \mathrm{O}\left(r^{2}=0.9939 ;\right.$ Fig 6.2A).

By establishing the standard curves for each condition in the respective media, the accuracy of the assay was improved. Using an LB-based standard curve the measured concentration of ATP in LB supplemented with $500 \mu \mathrm{M}$ of ATP was $487.6 \pm 3.7 \mu \mathrm{M}$, while for a similar approach for RPM1 yielded a value of $501.5 \pm 18.5 \mu \mathrm{M}$ (error values are 
reported as the standard deviation of two biological replicates, each comprised of three technical replicates, and derived from the standard curves pictured in Fig. 6.3.

Establishing standard curves in the media to be tested proved to be more accurate than establishing the standard curves in $\mathrm{dd}_{2} \mathrm{O}$ and was adopted as the preferred method going forwards.

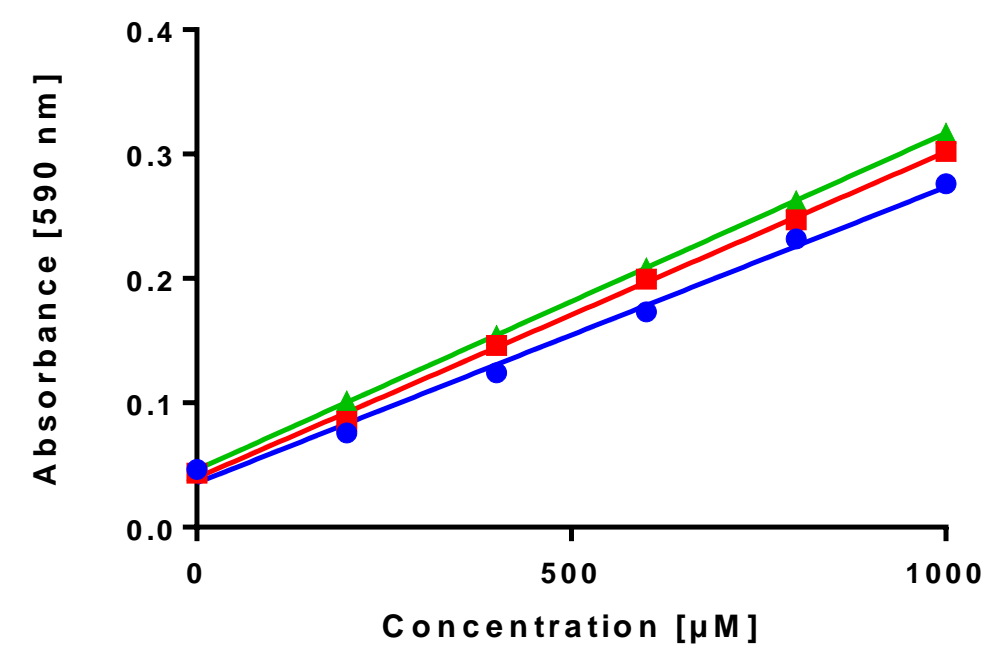

Figure 6.3 Comparison of standard curves between $\mathrm{ddH}_{2} \mathrm{O}, \mathrm{RPMI}$ and LB: The standard curves were established using the optimised protocol described in methods Section 2.10.6. GraphPad Prism ${ }^{\circledR}$ was used to calculate the $r^{2}$ values for each line. RPMI (red line) was slightly more linear and reached a slightly higher $A_{590}$ compared to LB (blue line).

Standards set up in $\mathrm{ddH}_{2} \mathrm{O}$ (green line) are also displayed. Data is the average of three replicates.

The underlying reason for the assay not performing as well in $\mathrm{LB}$ as $d d \mathrm{H}_{2} \mathrm{O}$ was not elucidated despite the improvement in assay performance. As similar effects were seen with measurement of L-glutamine depicted in Figure 5.7 it is unlikely a component of the LB is interfering directly with the compound being measured. Additionally, it can be seen from Figure 6.3 that all of the data points in the standard curve for LB were consistently lower than the data points for either the $\mathrm{ddH}_{2} \mathrm{O}$ or RPMI media. As such, it did not seem likely that LB was slowing the reaction velocity down enough to stop all of the ATP being consumed, as if this had been the case it would be expected that the higher concentrations would be more affected than the low concentrations. To rule out LB causing the reaction to have a different $\mathrm{pH}$, the $\mathrm{pH}$ of the reaction was tested $(25 \% \mathrm{v} / \mathrm{v}$ 
LB with $75 \% \mathrm{v} / \mathrm{v}$ buffered reaction $\mathrm{mix}$ ). The $\mathrm{pH}$ was determined to be 8.5 , indicating that the reduction in $\mathrm{A}_{590}$ values was not $\mathrm{pH}$ related.

The change in $\mathrm{A}_{590}$ values over time was measured in both $\mathrm{dd}_{2} \mathrm{O}$ and $\mathrm{LB}$ using the same protocol as described in methods Section 2.10.2 with two modifications, that the concentration of BpsA was reduced to $0.5 \mu \mathrm{M}$, decreasing the reaction speed to allow for a more accurate examination of the curve and L-glutamine was added to the reaction mix while ATP was removed. To initiate the reaction, ATP at a concentration of $4 \mathrm{mM}$ in $25 \mu \mathrm{L}$ of either LB or $\mathrm{ddH}_{2} \mathrm{O}$ was added to $75 \mu \mathrm{L}$ of reaction mix. This was done to mirror the same ratio of LB to reaction mix as used in the DMSO stop assay described in Section 6.3. As illustrated in Fig 6.4 , the $V_{\max }$ was slower for the samples containing $25 \%$ LB than $\mathrm{dd}_{2} \mathrm{O}$. The characteristic indigoidine precipitation curve also appears to be less pronounced. This phenomenon has been seen previously for samples exhibiting a much lower overall rate of indigoidine synthesis, for example as per the kinetic analysis of different L-glutamine levels in Section 5.5. However, the more rapid increase in initial $A_{590}$ values has not been observed before. These data may indicate that a component in LB was interfering with the synthesis of indigoidine.

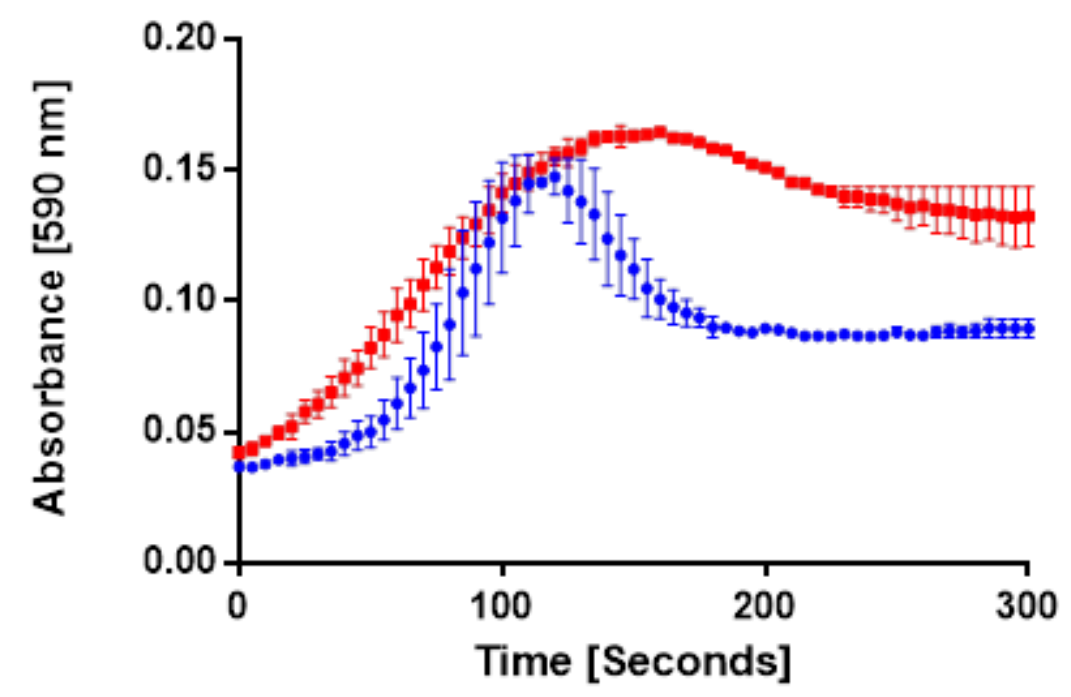

Figure 6.4 Comparison of reaction velocity of a sample supplemented with $\mathbf{2 5} \%$ LB vs no supplementation: The reaction was initiated by the addition of $25 \mu \mathrm{L}$ of $4 \mathrm{mM}$ ATP either in LB (red squares) or dd $\mathrm{H}_{2} \mathrm{O}$ (blue circles) to $75 \mu \mathrm{L}$ of reaction mix with BpsA at a concentration of $0.5 \mu \mathrm{M}$. The $\mathrm{V}_{\max }$ of the reaction is higher for $\mathrm{ddH}_{2} \mathrm{O}$. Data points are the average of two biological replicates each comprising of three technical replicates. Error bars represent \pm SEM.

To determine whether any primary component in the LB was interfering with the reaction velocity, the three un-autoclaved constitutive components were also tested 
individually (i.e., $0.5 \% \mathrm{w} / \mathrm{v} \mathrm{NaCl}, 0.5 \% \mathrm{w} / \mathrm{v}$ yeast extract and $1.0 \% \mathrm{w} / \mathrm{v}$ tryptone). The assay was set up in the same manner as previously described in Fig 6.4 with each component being tested supplemented with $4 \mathrm{mM}$ ATP to initiate the reaction. As illustrated in Fig 6.5A none of the individual components had a substantial effect on the reaction velocity relative to the $\mathrm{ddH}_{2} \mathrm{O}$ control. When the $\mathrm{A}_{590}$ values were plotted for each LB component in the same manner as in Fig 6.4, the yeast extract had a curve that was more similar to the $\mathrm{LB}$ when compared to the $\mathrm{NaCl}, \mathrm{ddH}_{2} \mathrm{O}$ and tryptone samples (data not shown). It is possible that it could be a synergistic effect between different compounds causing the reduced reaction velocity in LB. Moreover, while the reagents were all the same brand, the exact method for synthesising the LB could be different, resulting in different curves.

Another difference between the $\mathrm{NaCl}$, yeast extract and tryptone and the premade LB was that the LB was autoclaved. This could potentially modify the chemical constituents and cause it to have a different chemical composition to the unautoclaved components. To determine if this was the case un-autoclaved LB was compared to autoclaved LB in the same manner as Fig 6.4. As illustrated in figure 6.5B, non-autoclaved media had a slightly higher $V_{\max }$ than autoclaved media and the shape of the curve was similar to that of $\mathrm{ddH}_{2} \mathrm{O}$. This suggests that a chemical change occurs during the autoclaving process, which has a detrimental effect on the synthesis of indigoidine. 
A

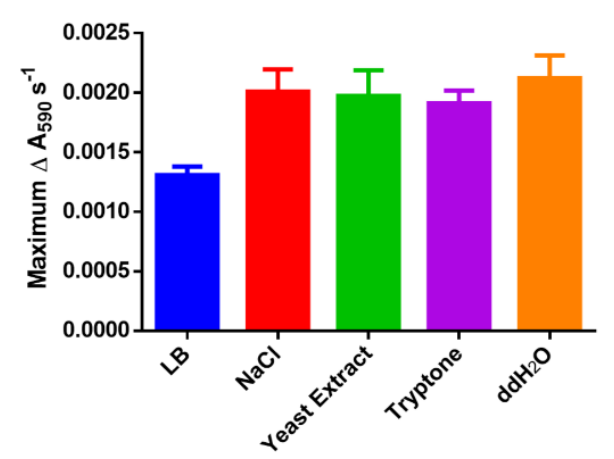

B

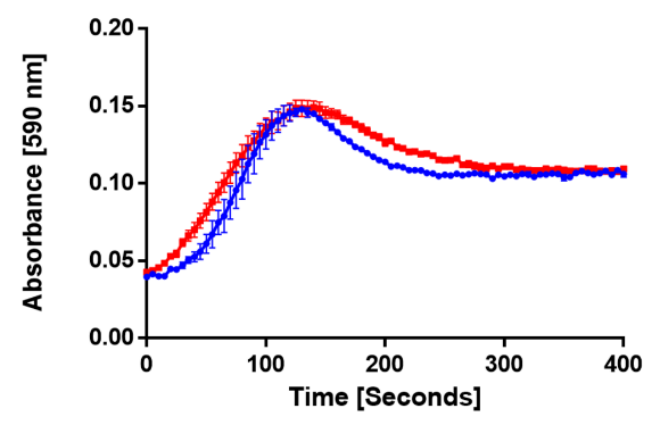

Figure 6.5 Maximum reaction velocity for LB components and the effect of autoclaved vs non-autoclaved LB on reaction velocity: (A) The reaction was initiated by the addition of $25 \mu \mathrm{L}$ of $4 \mathrm{mM}$ ATP present in either autoclaved LB, unautoclaved $\mathrm{NaCl}$, unautoclaved Yeast extract, unautoclaved Tryptone or $\mathrm{dd}_{2} \mathrm{O}$ condition. The $\mathrm{A}_{590}$ value was monitored every five seconds and the Excel ${ }^{\circledR}$ slope function was used to derive the slope every five data points. The maximum slope for each reaction was then identified. Data is the average of two biological replicates each comprising of three technical replicates. Error bars represent \pm SEM (B). The reaction was initiated by the addition of $25 \mu \mathrm{L}$ of 4 mM ATP either in autoclaved LB (red squares) or non-autoclaved LB (blue circles) to $75 \mu \mathrm{L}$ of reaction mix with BpsA at a concentration of $0.5 \mu \mathrm{M}$. Data points are the average of two biological replicates each comprising of three technical replicates. Error bars represent \pm SEM.

The slower reaction velocity still does not explain why the lower ATP concentration samples also had lower $A_{590}$ readings since the hour incubation should still be sufficient to convert all of ATP into indigoidine. If the reaction velocity was the cause of the reduced $A_{590}$ values we would expect to see more of curve rather than a linear line with the standards in LB as all of the ATP at higher concentrations would not have been consumed.

With changes in $\mathrm{pH}$ and reaction velocity unlikely to be the sole cause of the reduced $A_{590}$ values another source could be a component in the LB reducing the indigoidine. Previous work by Muller et al has shown that the addition of a stronger antioxidant such as ascorbic acid to the DMSO could prevent the indigoidine from converting in the colourless leuco form (Müller et al., 2012). Therefore, the protective action of ascorbic acid could potentially stop the degradation of indigoidine. However, when ascorbic acid was added to either the reaction mix or the DMSO used to resolubilise the indigoidine no improvement in assay performance was observed (Fig. 6.6). 


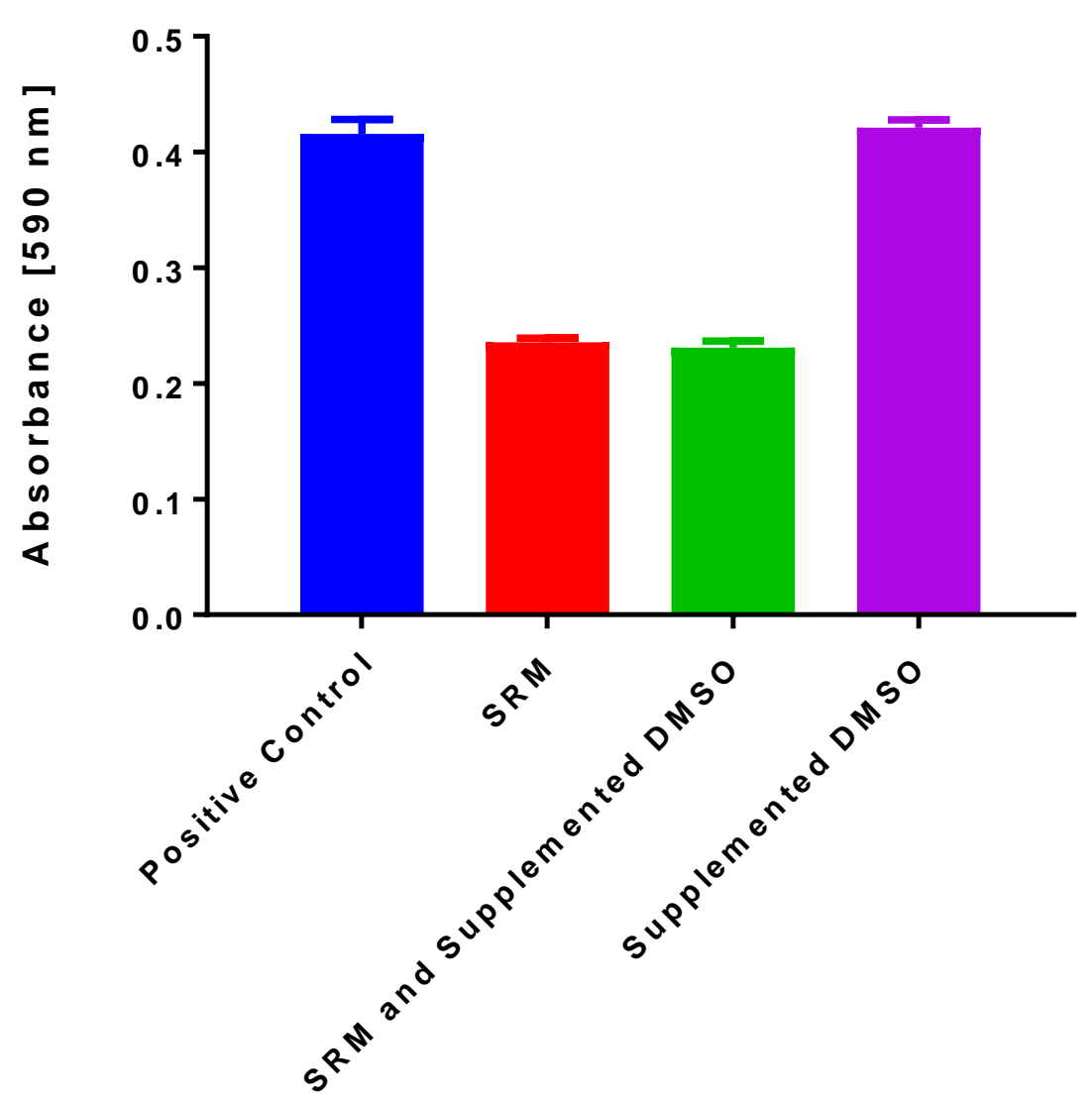

Figure 6.6 The effect of the addition of $5 \mathrm{mM}$ ascorbic acid on total indigoidine production: The addition of $5 \mathrm{mM}$ ascorbic acid to the reaction mix ( red bar, labelled SRM) has a detrimental effect on the production of indigoidine. The addition of DMSO supplemented with ascorbic acid (green bar) does not increase the $A_{590}$ value. While the addition of $5 \mathrm{mM}$ ascorbic acid to the DMSO (purple bar, labelled supplemented DMSO) used to resolubilise the indigoidine has no effect. Data is the average of three replicates. Error bars represent \pm SEM.

\subsection{Detection of adenylation by an NRPS A domain}

\subsubsection{Design of an NRPS substrate specificity assay}

The assay to detect adenylation using BpsA is comprised of two steps. Firstly, the purified NRPS or A domain construct is incubated with the amino acids to be assayed for activity along with $\mathrm{MgCl}_{2}$, ATP and buffer at $30^{\circ} \mathrm{C}$ for $30 \mathrm{~min}$ to allow adenylation to occur after which a mix containing the reagents necessary for BpsA to synthesise ATP is added. The reaction is then incubated for a further half an hour to allow indigoidine synthesis to occur and the total amount of indigoidine produced is measured. Since Lglutamine is in excess, indigoidine synthesis is dependent on the fixed amount of ATP present in the sample, therefore if adenylation has occurred there will be a reduction in the amount of ATP present resulting in a reduced $A_{590}$ value for that sample. 


\subsubsection{Detection of adenylation using the EntF A domain}

To determine if it was possible to measure adenylation by monitoring the consumption of ATP the A domain from EntF, a NRPS in the enterobactin biosynthesis pathway in $E$. coli. The EntF A domain was purified using the standard purification protocol described in Section 2.5.2 with the following modifications; the growth media was supplemented with both betaine and sorbitol. Dr Owen found that if the media wasn't supplemented in this manner the A domains often purified out in a soluble but slightly misfolded form, which resulted in off target adenylation. The EntF A domain was stored in a Tris based buffer as storage in a sodium phosphate buffer would give interfere with the malachite green assay. An SDS-PAGE gel was then used to confirm the purity and solubility of the protein. High yields were obtained with approximately $30 \mathrm{mg}$ of protein from $400 \mathrm{ml}$ of protein culture (Fig. 6.7A).

A modified version of the malachite green assay was then used to determine if the purified EntF was selective for L-serine, its native substrate, which would confirm it had been correctly folded. Six reactions where set up using the method described in Section 2.11.1 The EntF A domain was strongly selective for L-serine and had virtually no activity with either L-glutamine or L-tryptophan when compared to the addition of no amino acid. (Fig. 6.7B). This indicated that the EntF A domain was correctly folded and was therefore a good candidate to determine if the ATP assay was a viable method to detect adenylation. 
A

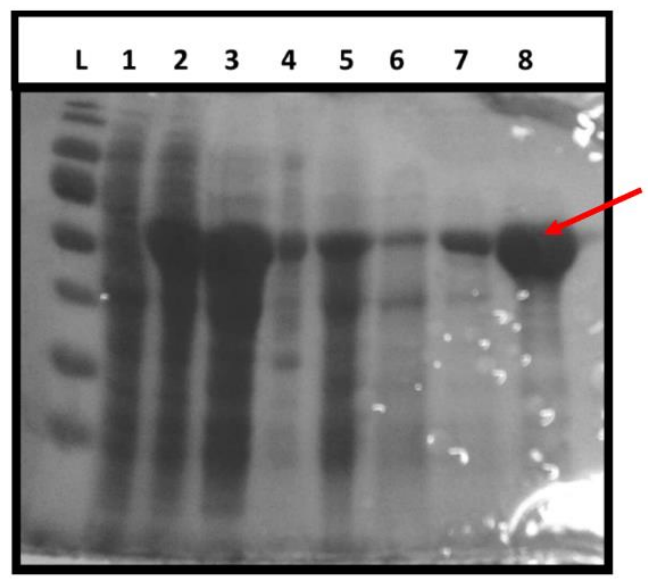

B

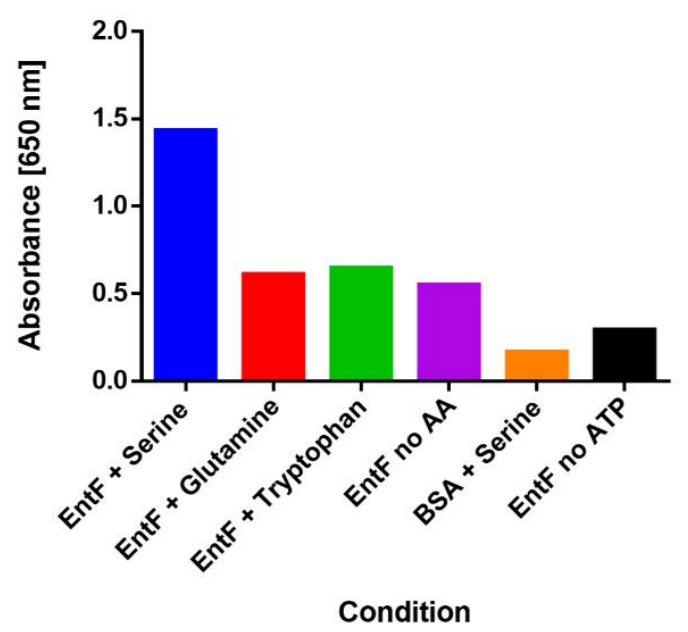

Figure 6.7 Determination of the EntF A domain construct solubility and activity: (A) A $12 \%$ SDS-PAGE gel showing the expression and purification of soluble EntF A domain. 1) before induction sample 2) $24 \mathrm{~h}$ post induction sample, showing a clear band at approximately $55 \mathrm{kDa}$. 3) Soluble fraction following lysis 4) Insoluble fraction following lysis 5) Elution collected after sample applied to the column 6) Elution collected after bind buffer was applied to the column 7) Elution collected after wash buffer was applied to the column 8) Elution collected after elution buffer was applied to the column. The red arrow indicates the EntF A domain in the eluted fraction. Twelve $\mu \mathrm{L}$ of sample, resuspended in $3 X$ SDS loading buffer was added to each lane. (B) Malachite green assay illustrating that the EntF A domain is correctly folded and capable of adenylating serine, its native substrate. Bars represent one replicate with $40 \mathrm{\mu g}$ of EntF A domain added to the reaction.

If adenylation was occurring in the assay, a reduction in the amount of ATP present should occur, resulting in a reduced $A_{590}$ signal. In order to facilitate adenylation, $40 \mu \mathrm{g}$ of EntF A domain was incubated at $30^{\circ} \mathrm{C}$ for $30 \mathrm{~min}$ at $200 \mathrm{rev} / \mathrm{min}$ using the protocol described in methods Section 2.11.2 with the inorganic pyrophosphatase removed. The ATP concentration was fixed at $500 \mu \mathrm{M}$. At this concentration ATP provides a strong signal that is detectable within the linear range of the assay, but should still be a rate limiting step in the adenylation reaction. After the incubation step a master mix containing $3 \mu \mathrm{M}$ holo-BpsA, $50 \mathrm{mM}$ Tris- $\mathrm{Cl} \mathrm{pH} \mathrm{8.5,} 10 \mathrm{mM} \mathrm{MgCl}_{2}$ and $4 \mathrm{mM} \mathrm{L-Gln}$ in a final volume of $20 \mu \mathrm{L}$ was added to each well to enable the synthesis of indigoidine. The plate was then incubated for a further $1 \mathrm{~h}$ at $25^{\circ} \mathrm{C}$. The indigoidine was then resolublised by the addition of $200 \mu \mathrm{L}$ DMSO and the $A_{590}$ value was recorded. As illustrated in Fig. 6.8 no difference in indigoidine production was detected between the three amino acids ( $P$ value $>0.05$ using a two tailed $t$ test). The Bovine Serum Albumin (BSA) control had more indigoidine produced compared to the EntF samples and controls. This phenomenon was also observed using the malachite green assay, 
indicating that the EntF A domain consumes a low level of ATP without needing any amino acid.

To try and improve the sensitivity of the assay, the amount of EntF was increased up to $100 \mu \mathrm{g}$ and the incubation time to $1 \mathrm{~h}$, to ensure that it was not an issue with the reaction proceeding too slowly. Despite the increase in enzyme and incubation time no detectable difference in signal was observed (data not shown).

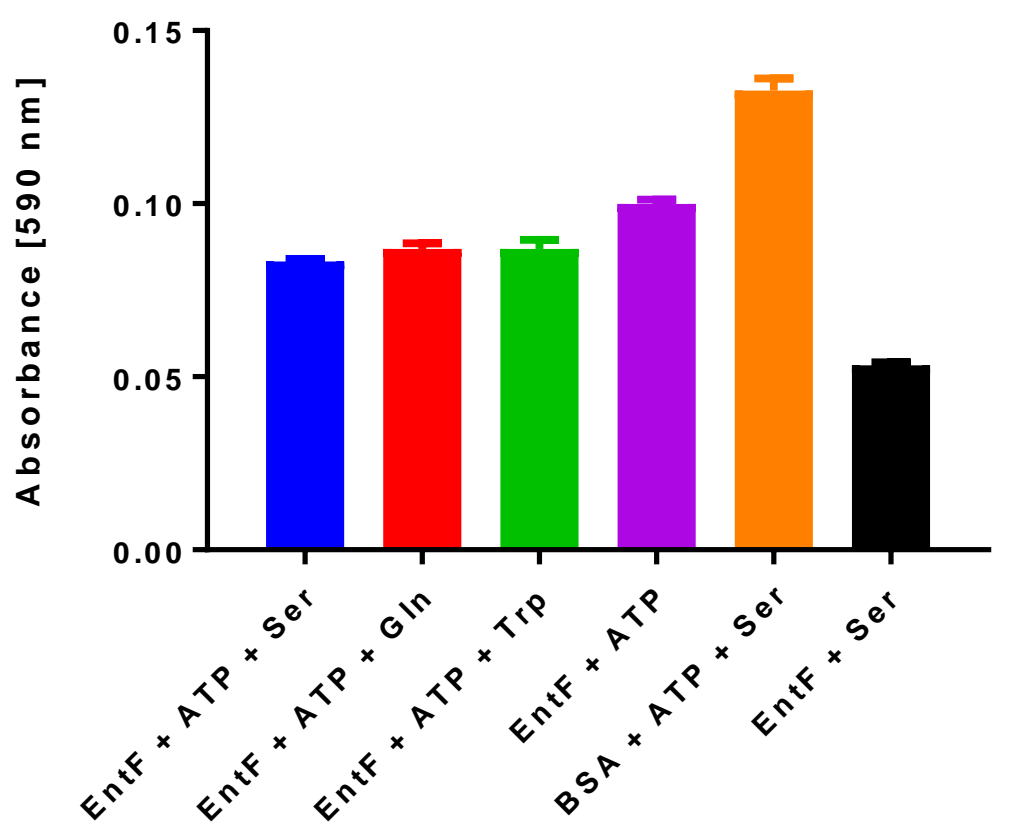

Figure 6.8 First attempt to measure adenylation by monitoring the consumption of ATP: A reaction mix containing $40 \mu \mathrm{g}$ of enzyme, $\mathrm{MgCl}_{2}$, Tris- $\mathrm{Cl} \mathrm{pH} \mathrm{7.8,} \mathrm{amino} \mathrm{acid} \mathrm{(either} \mathrm{serine} \mathrm{(blue} \mathrm{bar),} \mathrm{glutamine(} \mathrm{red} \mathrm{bar)} \mathrm{or} \mathrm{tryptophan} \mathrm{(green}$ bar) and ATP was incubated at $30{ }^{\circ} \mathrm{C}$ for 30 min to bring about the consumption of ATP through the adenylation reaction. A second reaction mix containing $\mathrm{BpsA}, \mathrm{MgCl}_{2}$, Tris- $\mathrm{Cl} \mathrm{pH} 7.8$ and L-glutamine was then added. The total amount of indigoidine synthesised was then determined. There was no statistical significant difference between the substrate of EntF, serine and other amino acids indicating adenylation was not being detected. Data is the average of three replicates and error bars represent \pm SEM.

One reason for the EntF A domain not consuming a detectable amount of ATP when it was exposed to serine compared to the other amino acids could be due to the nature of the adenylation reaction. Since the adenylation reaction is an equilibrium reaction, the reaction products need to be removed from the reaction in order to drive it towards the consumption of ATP. In the assay's current form, the adenylated amino acid or the PP is not being removed or converted into another product. Depending on where the equilibrium of the reaction sits this could result in only a small amount of ATP being consumed before equilibrium is reached. 
To avoid the equilibrium issue the malachite green assay uses the enzyme pyrophosphatase to convert the $\mathrm{PP}_{\mathrm{I}}$ to $\mathrm{P}_{\mathrm{i}}$ resulting in the adenylation reaction not reaching equilibrium and $\mathrm{PP}_{\mathrm{i}}$ continually being produced. I reasoned that pyrophosphatase could also be used in this assay to perform the same role, and that the removal of $\mathrm{PP}_{\mathrm{i}}$ from the reaction might drive the consumption of ATP.

To determine if this was the case, pyrophosphatase at a concentration of $0.03 \mathrm{U}$ was added to the $30 \mu \mathrm{L}$ of reaction mix as described in Section 2.13.2. As illustrated in Fig. 6.9 the pyrophosphatase pushed the equilibrium towards the consumption of ATP, which allowed enough ATP to be consumed to be detected by the assay. After $30 \mathrm{~min}$, the EntF A domain + serine + ATP sample had all of the ATP present consumed and there was no statistically significant difference in the amount of end-product (indigoidine) as the no ATP control $(P>0.05)$. Interestingly the same pattern of higher ATP consumption with the EntF A domain compared to the BSA control was preserved, indicating that nonspecific ATP consumption happens with this A domain. Both the tryptophan and glutamine controls did not have significantly different $(P>0.05)$ values to the no amino acid control, indicating that the EntF A domain has no selectivity for either of these amino acids. This is in agreement with the literature (Owen, 2010) and the results from Fig. 6.7. 


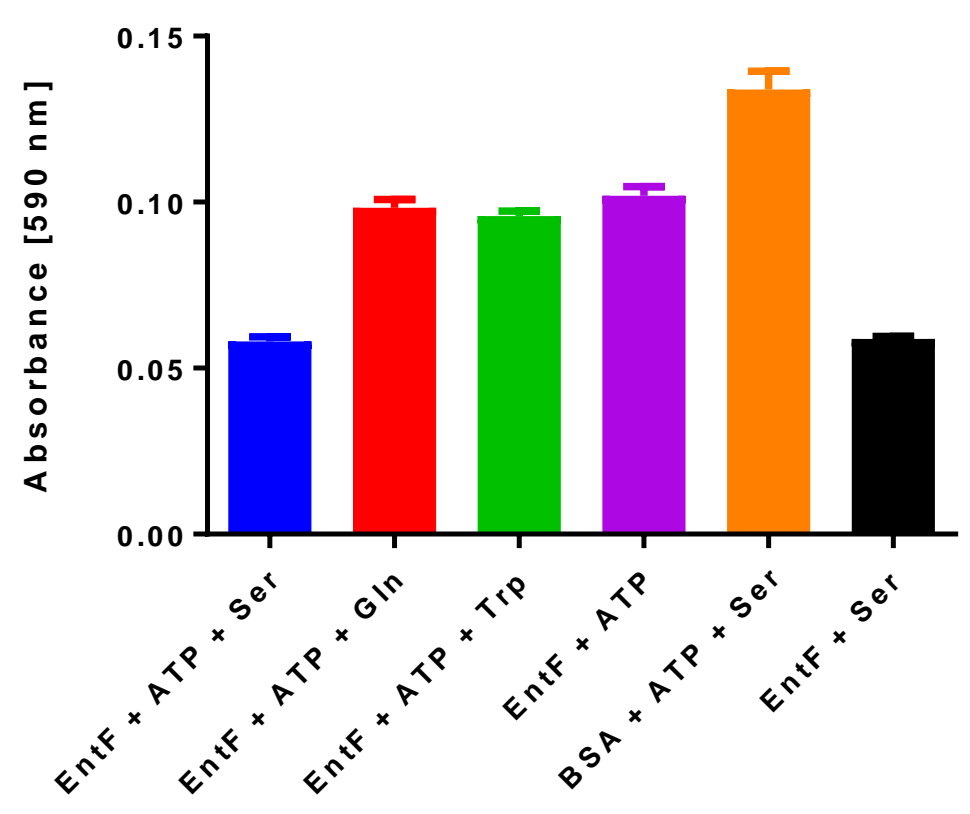

Figure 6.9 The addition of pyrophosphatase results in detectable adenylation: A reaction mix containing $100 \mu \mathrm{g}$ of enzyme, $\mathrm{MgCl}_{2}$, Tris- $\mathrm{Cl} \mathrm{pH} \mathrm{7.8,} \mathrm{amino} \mathrm{acid} \mathrm{(either} \mathrm{serine} \mathrm{(blue} \mathrm{bar),} \mathrm{glutamine(} \mathrm{red} \mathrm{bar)} \mathrm{or} \mathrm{tryptophan} \mathrm{(green} \mathrm{bar),} 0.03$ $\mathrm{U}$ pyrophosphatase and ATP was incubated at $30^{\circ} \mathrm{C}$ for $30 \mathrm{~min}$ to bring about the consumption of ATP through the adenylation reaction. A second reaction mix containing $\mathrm{BpsA}, \mathrm{MgCl}_{2}$, Tris- $\mathrm{Cl} \mathrm{pH} 7.8$ and L-glutamine was then added. The total amount of indigoidine synthesised was then determined. Data is the average of three biological replicates and error bars represent \pm SEM.

This assay represents a novel approach to determining A domain substrate specificity by monitoring ATP consumption rather than the production of $\mathrm{PP}_{\mathrm{i}}$. It also further illustrates the flexible and elegant nature of BpsA and its ability to be adapted to wide variety of uses as a reporter.

\subsection{Synthesis and detection of a novel indigoidine analogue}

In addition to the native substrate of BpsA, L-glutamine, Dr Ackerley and I postulated that glutamine analogues may also be accepted by the adenylation domain and potentially be converted into an indigoidine like compound. Glutamine analogues are commonly used in biotechnology, for example the dipeptide alanyl-glutamine is a common cell culture reagent due to its improved solubility and stability compared to glutamine (Imamoto et al., 2013) and for the same reasons alanyl-glutamine is also commonly used in wound-healing applications (Déchelotte et al., 2006).

Being able to detect and measure alanyl-glutamine using BpsA might be useful, as current methods are cumbersome and there does not appear to be a colorimetric kit 
available on the market (Krömer et al., 2011). Indigoidine also has mild anti-microbial properties (Cude et al., 2012), so it was reasoned that there is the potential for indigoidine analogues to have improved antimicrobial properties or pharmacokinetic profiles. Testing the ability of BpsA to process different glutamine analogues might also provide a method for evaluating the flexibility of the A domain active site and its tolerance for bulky substrates, and insight into the tolerances of the PCP and TE domain. Finally, there has been some research into using indigoidine as an industrial dye. It is possible that indigoidine analogues may have improved solubility and/or stability, increasing their appeal as commercial dyes (Xu et al., 2015).

Work by Villiers et al (2009) has shown that the A domain of the NRPS TycA can accept and adenylate a variety of different substrates, including the dipeptide Gly-L-Phe. The relative catalytic efficiency with Gly-L-Phe was substantially less $\left(6.8 \times 10^{-5}\right)$ than the native amino acid L-Phe (Villiers and Hollfelder, 2009). Substrate acceptance by the TycA A domain appears to be controlled by a variety of conditions including hydrophobic effects, shape complementarity and steric exclusion (Villiers and Hollfelder, 2009). Based on the findings of Villiers et al it was hoped that the BpsA A domain would display a level of promiscuity and the downstream catalytic domains would be accepting of the analogue.

\subsubsection{Synthesis of an indigoidine analogue from alanyl-glutamine}

The empirical formula of alanyl glutamine is $\mathrm{C}_{8} \mathrm{H}_{15} \mathrm{~N}_{3} \mathrm{O}_{4}$ and has a molecular weight of 217.22 (see structure in Fig 6.10A). It was reasoned that the position of an associated amino acid such as alanine at the $\mathrm{N}$-terminal position of the dipeptide should still allow the synthesis of an indigoidine like structure (from here onwards identified as alaindigoidine), as illustrated in Fig. 6.10C. To determine if ala-indigoidine could be produced, a reaction was set up as described in Section 2.12.1 using $1 \mu \mathrm{M}$ of holo BpsA and $4 \mathrm{mM}$ of alanyl-glutamine. However, after a $1 \mathrm{~h}$ incubation period, no detectable pigment production was observed. 
A<smiles>NC(=O)CCC(N)C(=O)O</smiles><smiles>C[C@H](N)NC(CCC(N)=O)C(=O)O</smiles>

B<smiles>NC1=CC(=C2C=C(N)C(=O)NC2=O)C(=O)NC1=O</smiles>

C<smiles>COC(=O)NC1=CC(=C2C=C(NC(=O)O)C(=O)NC2=O)C(=O)NC1=O</smiles>

Figure 6.10: Structures of glutamine, alanyl-glutamine, Indigoidine and proposed indigoidine analogue: (A) Glutamine (top) and alanyl-glutamine (bottom) are adapted from the Sigma Aldrich website. (B) The structure of indigoidine, Chemdraw ${ }^{\circledR}$ was used to illustrate and clean the structure. (C) The predicted structure of the alanylglutamine analogue (ala-indigoidine). Chemdraw ${ }^{\circledR}$ was used to illustrate and clean the structure.

Since the catalytic efficiency of the TycA A domain with its dipeptide (Villiers and Hollfelder, 2009) was substantially slower than with the native phenylalanine substrate, I reasoned that this might also be the case with BpsA and alanyl-glutamine. I therefore increased the concentration of substrate to $10 \mathrm{mM}$, that of BpsA to $12 \mu \mathrm{M}$, and the incubation time to $16 \mathrm{~h}$ at $25^{\circ} \mathrm{C}$. This resulted in the synthesis of a visible blue pigment from the sample containing alanyl-glutamine.

I first considered that this pigment might just be indigoidine, formed from glutamine as a low-level contaminant or breakdown product of the alanyl-glutamine. However, the pigment appeared to be more soluble in the buffered reaction mix than indigoidine synthesised from L-glutamine (Fig 6.11A). An absorbance spectrum scan of the aqueous fraction from each of the two samples had slightly different peaks. The alanyl-glutamine derived sample had a maximum absorbance of 0.843 at a peak of $608.5 \mathrm{~nm}$, whereas the L-glutamine derived sample had a maximum absorbance of 0.387 at a peak of $590 \mathrm{~nm}$ when measured in a Shimadzu UV-1800 spectrophotometer between the wavelengths of 400 and $800 \mathrm{~nm}$ with $0.5 \mathrm{~nm}$ intervals.

It is known that the maximum absorbance of indigoidine changes depending on the solvent. In DMSO, the solvent in which indigoidine has the highest solubility, it has a maximum absorbance at $613 \mathrm{~nm}$ (Takahashi et al., 2007). It is possible that alaindigoidine has a higher extinction coefficient than indigoidine, alternatively the maximal absorbance at a higher wavelength suggests that the ala-indigoidine might be 
more soluble in water than indigoidine. A further possibility, albeit unlikely given the level of pigment produced, is that the presence of unreacted alanyl-glutamine might simply be altering the solvent conditions and thereby affecting the absorbance spectrum of normal indigoidine formed from an L-glutamine contaminant or breakdown product. In isolation, the formation of a blue pigment is not conclusive evidence that a different species of indigoidine is being formed. Sigma Aldrich ${ }^{\circledR}$ lot analysis of the alanylglutamine reports it being $>98 \%$ pure but the contaminating species are not described. If L-glutamine is present as a low-level contaminant, or if alanyl-glutamine is able to hydrolyse overnight to release L-glutamine, then this could lead to production of regular indigoidine by BpsA. Moreover, in this scenario it is possible that alanyl-glutamine might be competing for the A domain active site but being unable to be synthesised into indigoidine, which could explain why the reaction velocity for the low levels of Lglutamine present was greatly reduced.

To determine if low levels of L-glutamine produced a similar absorbance reading and had similar properties to the sample containing alanyl-glutamine, the $\mathrm{A}_{590}$ value of $80 \mu \mathrm{M} \mathrm{L-}$ glutamine (the equivalent of $2 \%$ of $4 \mathrm{mM}$ alanyl-glutamine) was monitored at $590 \mathrm{~nm}$ over 15 hours and directly compared to a parallel reaction containing $4 \mathrm{mM}$ alanylglutamine. The $80 \mu \mathrm{M}$ L-glutamine was found to exhibit the characteristic indigoidine curve with a rapid increase in $A_{590}$ values then a decrease as indigoidine dropped out of solution. In contrast, the $4 \mathrm{mM}$ alanyl-glutamine sample had a much more gradual increase in absorbance. It also did not have the same characteristic decline indicating that it was dropping out of solution (Fig. 6.11B).

The different maximal absorbance wavelength and the differences in the $A_{590}$ value over time strongly suggest that a different compound is being synthesised by BpsA from alanyl-glutamine. In order to provide further supporting evidence, indigoidine and alaindigoidine were analysed by HPLC to compare their retention times. 
A

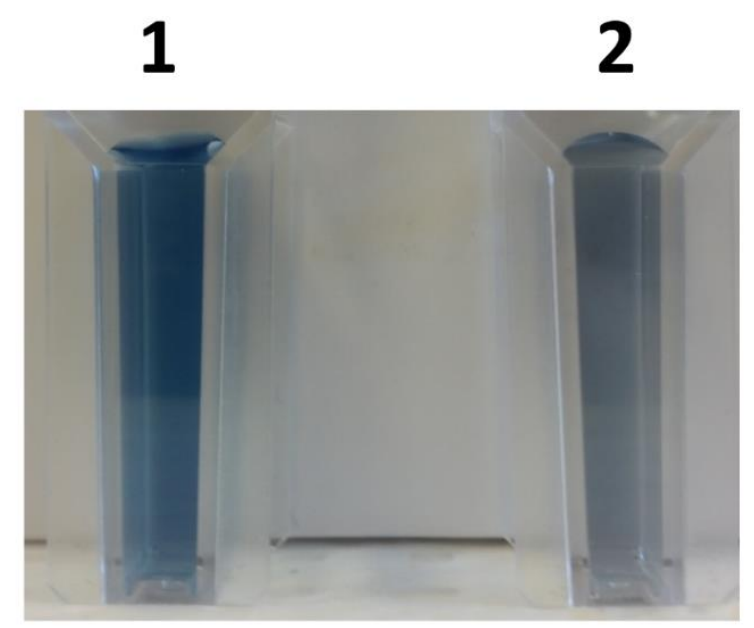

B

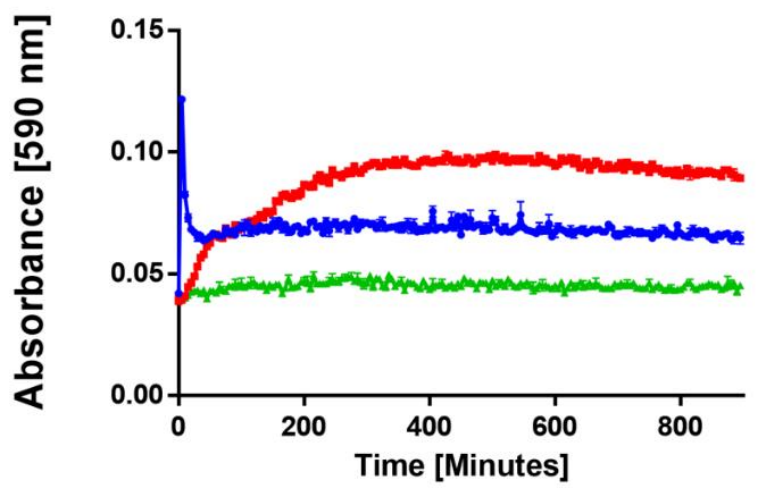

Figure 6.11 different in the synthesis patterns of indigoidine synthesised from glutamine and alanyl-glutamine: (A) ala-indigoidine synthesised from alanyl-glutamine (left tube labelled 1) appeared to be more soluble than indigoidine synthesised from L-glutamine (right tube labelled 2) in the reaction mix containing dd $\mathrm{H}_{2} \mathrm{O}, 50 \mathrm{mM} \mathrm{Tris-Cl} \mathrm{pH} \mathrm{8.5,}$ $\mathrm{MgCl}_{2}$, ATP and $4 \mu \mathrm{M}$ BpsA. (B) The absorbance was recorded at $590 \mathrm{~nm}$ every 5 min over $15 \mathrm{~h} .80 \mu \mathrm{M}$ L-Glutamine (blue squares) shows the characteristic indigoidine curve. $4 \mathrm{mM}$ alanyl-glutamine (red circles) slowly increases in absorbance before levelling off. The no glutamine negative control (green line) did not exhibit an increase in $A_{590}$. Data is the average of three replicates and error bars represent \pm SEM.

\subsubsection{Detection of the indigoidine analogue using HPLC}

An Agilent HPLC was used with a $\mathrm{C}_{18}$ column. $800 \mu \mathrm{L}$ of the master mix described in Section 2.12.1 was incubated for 16 hours at $25^{\circ} \mathrm{C}$ to bring about the synthesis of indigoidine and ala-indigoidine. The indigoidine and ala-indigoidine was then resuspended in $200 \mu \mathrm{L}$ of $d_{d H_{2}} \mathrm{O}$ and $20 \mu \mathrm{L}$ of the sample was then loaded onto the 
column with the method described in Section 2.12.2. Detection was set up at $590 \mathrm{~nm}$ and two different retention peaks were observed indicating that two different compounds were being produced as illustrated in Fig. 6.12

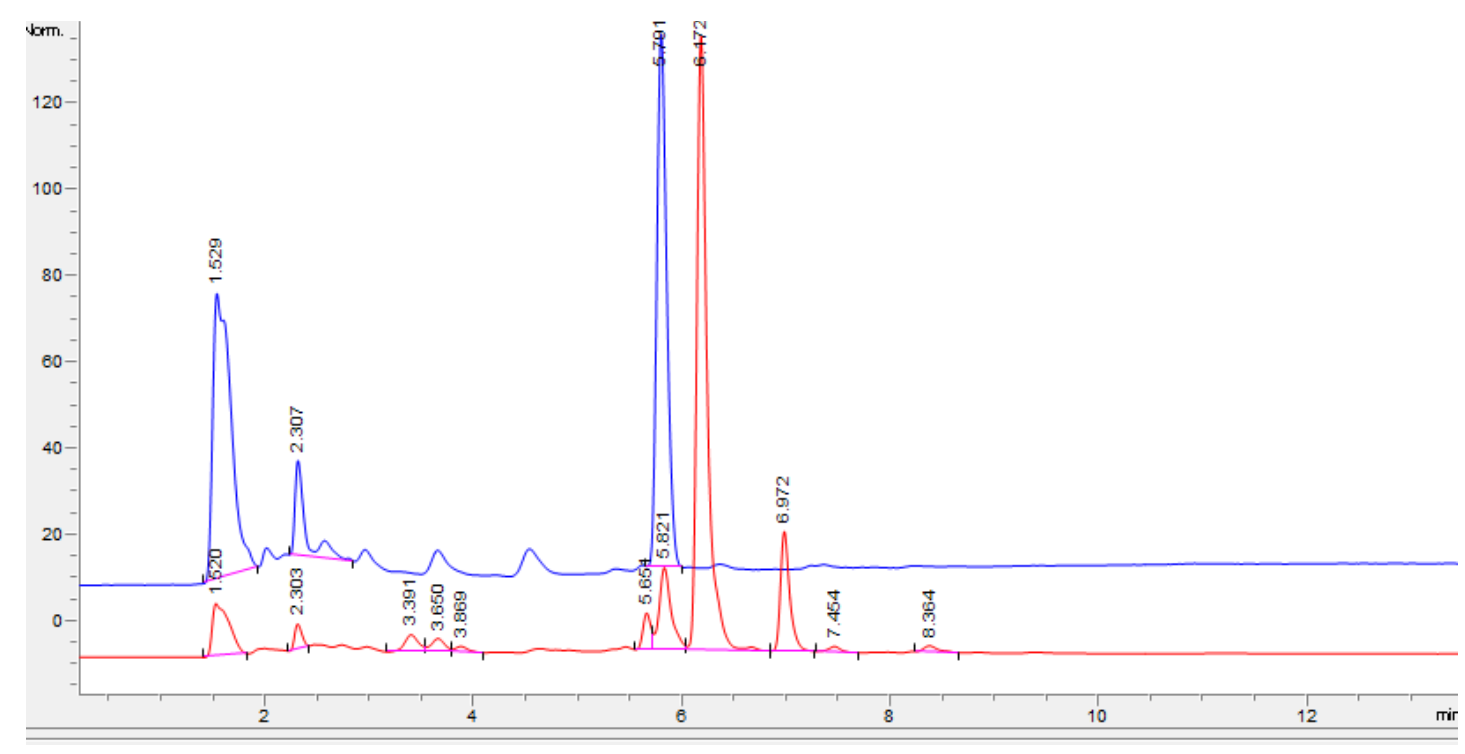

Figure 6.12 HPLC trace of indigoidine and ala-indigoidine: Indigoidine (blue) has an elution peak of 5.791 min while ala-indigoidine (red) has an elution peak of $6.172 \mathrm{~min}$. Detection is at $590 \mathrm{~nm}$. A second smaller peak is also present at $6.972 \mathrm{~min}$, indicating that a second species may also be synthesised.

While the HPLC traces provided strong evidence that two different compounds were being produced, the exact chemical structure of the putative ala-indigoidine compound remains unconfirmed. Even if derived at least in part from alanyl-glutamine, there was the potential for it to be a hybrid molecule with one part of the dimer being synthesised from glutamine and the other half being synthesised from alanyl-glutamine. This might explain why there are two peaks present in the ala-indigoidine sample in Fig 6.12. In order to try and confirm the structure of ala-indigoidine I attempted to measure molecular weight using LC-MS. However, I could not detect ala-indigoidine, the reasons for this is discussed further in Section 6.8.3. 


\subsection{Investigation of 4' oxalocrotonate tautomerase mediated conversion of indigoidine to the colourless leuco form}

The anti-microbial activity of indigoidine has been reported by two papers (Cude et al., 2012; Gromek et al., 2016). Despite this reported effect, indigoidine appears to be a poor antimicrobial compound due to its poor solubility in water. Cude et al (2012) proposed that the colourless leuco form was in fact the antimicrobial form, and indicated that a 4' oxalocrotonate tautomerase encoded by the igiF gene (RBY4I_618) present in the Roseobacterium Phaeobacter sp. Strain Y4I could potentially catalyse the conversion of indigoidine into colourless leuco-indigoidine (Fig 6.13A).

Cude et al (2012) showed that the in vivo production of indigoidine by Phaeobacter sp. Strain Y4I caused competitive surface colonization against a variety of species including a range of Vibrio species (Cude et al., 2012). This same anti-microbial activity was shown by Gromek et al (2016), in species of Leisingera. Cude et al and Gromek et al were unable to test the in vitro activity of the leuco indigoidine, due in part to the need for the maintained presence of a strong reducing agent that they reasoned might mask the antimicrobial properties. Whether or not Igif can convert indigoidine into leuco-indigoidine in vitro has not yet been published in the literature.

The 4' oxalocrotonate tautomerases are a family of proteins that catalyse the isomerisation of unsaturated ketones. The functional enzyme is a homo-hexamer comprised of a trimer of dimers, with six active sites (Whitman, 2002). A tautomerase gene homologous to igiF was identified in the S. lavendulae bioactive gene cluster by Li et al, with the accession number AHW58014.1 (Li et al., 2015). The S. lavendulae homologue of the igiF gene, which encodes a protein that shares $40.3 \%$ amino acid identity with IgiF from Phaeobacter, was used in this study.

\subsubsection{Cloning, expression and purification of IgIF}

The nucleotide sequence for S. lavendulae igiF was codon optimised for $E$. coli expression using the codon optimisation tool available on the IDT ${ }^{\circledR}$ website. It was then expressed and purified with a $6 \mathrm{His} \mathrm{N}$-terminus tag using the purification protocol described in Section 2.5.5.3 with the following modifications: Due to the relatively 
complex quaternary structure of the protein the wash step was excluded and the column was washed with $30 \mathrm{ml}$ of bind buffer. The protein was then desalted using a 10 kDa cut off column and stored in a pH 7.2 sodium phosphate buffer supplemented with $40 \% \mathrm{v} / \mathrm{v}$ glycerol at $-20^{\circ} \mathrm{C}$. Solubility and purity was confirmed by running the protein on a $17 \%$ SDS-PAGE gel. The final yield was $0.5 \mathrm{mg}$ from $400 \mathrm{ml}$ of culture.

\subsubsection{Testing the activity of IgiF}

To determine if S. lavendulae IgiF (hereafter referred to as IgiF) was capable of converting indigoidine into the colourless leuco-indigoidine form, a reaction was set up as described in Section 2.12.1, with the following modification: the $\mathrm{pH}$ of the reaction was adjusted to 7.8 , as other kinetic work with 4-oxalocrotonate tautomerases had used lower pH values compared to BpsA (Zandvoort et al., 2012), and the concentration of holo BpsA was reduced down to $0.5 \mu \mathrm{M}$ and negative controls supplemented with BSA in $40 \%(\mathrm{v} / \mathrm{v})$ glycerol to ensure the glycerol or protein content was not having an effect on the rate of indigoidine synthesis. As illustrated in figure 6.13B, supplementation with IgiF at a concentration of 4 or $16 \mu \mathrm{M}$ produced no difference in $A_{590}$ values, indicating that in vitro Igif does not have an effect on the conversion of indigoidine to leuco-indigoidine. 
A<smiles>CC1=CC(C)C=C(C2C=C(N)C(=O)NC2=O)C1=O</smiles>

B

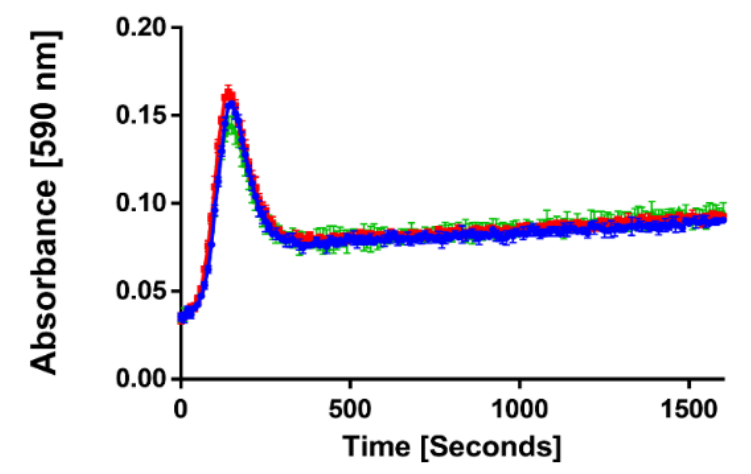

Figure 6.13 Conversion of indigoidine to leuco-indigoidine is potentially mediated by IgiF: (A) The proposed mechanism of conversion of indigoidine to leuco-indigoidine by Cude et al. (B) IgiF at a concentration of $4 \mu \mathrm{M}$ (blue line) and $16 \mu \mathrm{M}$ (red line) had no effect on the absorbance values of indigoidine when compared to $\mathrm{ddH}_{2} \mathrm{O}$ (green line). Data is the average of three replicates with error bars \pm SEM.

\subsection{Chapter discussion}

\subsubsection{Quantification of ATP}

The work described in this chapter sought to further illustrate the flexible nature of the BpsA biosensor. Building on the work in chapter five it repurposed the optimised assay to detect ATP in common bacterial and mammalian cell culture mediums. The effect of using the assay in different growth media was also probed, with a particular focus on the slight detrimental effect LB has on the assay. It was found that the effect LB has on the accuracy of the biosensor can be minimised by establishing the ATP standard curve in the media being tested, which is akin to "blanking" the assay. The effect of ascorbic acid on the assay was also investigated as it had been used in a previous paper to help 
stabilise the reaction. While ascorbic acid had a detrimental effect when added to the reaction mix it did not affect the reaction if added to the resolubilising DMSO.

A novel method for the detection of adenylation by A domains was also developed, which uses the consumption of ATP to determine if adenylation is occurring. The EntF A domain was purified and then tested for adenylation by using BpsA to report on the amount of ATP present in the incubated solution. Initial attempts failed due to an equilibrium state being reached and no more ATP being consumed. To overcome this, pyrophosphatase was added to the reaction mix which converted $\mathrm{PP}_{\mathrm{i}}$ into $\mathrm{P}_{\mathrm{i}}$, stopping the reaction from reaching equilibrium and enabling the $A$ domain to continue to adenylate the amino acid and consume ATP. The depletion of ATP could then be detected using the BpsA biosensor.

Being able to rapidly and accurately determine the concentration of ATP in variety of sample conditions is important for several different areas of research. While current kits on the market do an excellent job quantifying ATP, additional methods provide further flexibility to the user. This chapter focuses on the development of a biosensor to detect ATP in a range of different biologically relevant conditions. The quantification of ATP is very similar to that of L-glutamine in chapter five, which is to be expected as the key difference is essentially just swapping which substrate is in excess. The total time taken to run the assay was decreased from chapter five by increasing the BpsA concentration. This provides a point of difference to the colourimetric kits currently available on the market, and with some further optimisation it could be possible to run the entire assay in $20 \mathrm{~min}$.

The reason for the assay not performing as well in LB compared to water and RPMI media was also probed and it appears that autoclaving the yeast extract, which is by far the most complex of the components, produces a compound or compounds that interfere with the cyclised glutamine or indigoidine itself. If the measurement of Lglutamine was essential in LB, either establishing the standard curve in LB or sterilising the LB in another manner, for example by filter sterilising, may be applicable. The current ratio of reaction mix $(75 \% \mathrm{v} / \mathrm{v})$ to sample $(25 \% \mathrm{v} / \mathrm{v})$ is not fixed, so if reaction components are interfering with the assay it could be possible to change this ratio and 
still detect either ATP or L-glutamine. The only limit on the reaction size is the minimum percentage of DMSO needed to resolubilise the indigoidine.

\subsubsection{Quantification of adenylation}

A novel method for measuring adenylation was developed. This method used the consumption of ATP as a surrogate measurement for adenylation and could successfully detect the amino acid substrate specific of EntF A domain. By measuring the consumption of ATP rather than the production of $\mathrm{P}_{\mathrm{i}}$ or radiolabelled ATP we present another useful method. Initial attempts to measure the substrate specificity of the EntF A domain failed, due to the reaction dynamics. After a period of optimisation (attempts including modifying the enzyme concentration, ATP concentration and reaction time and temperature) it was determined that the reaction dynamics were responsible for the undetectable signal. By adding pyrophosphatase we were able to drive the reaction forwards and consume all of the ATP present. The method developed in this chapter for detecting substrate specificity is cheap and amendable to high throughput screening. However, it does still require a pyrophosphatase to convert $\mathrm{PP}_{\mathrm{i}}$ in to $\mathrm{P}_{\mathrm{i}}$. Further optimisation could improve the sensitivity and accuracy of this assay. This could include increasing the concentration of ATP causing more ATP to be consumed, resulting in a bigger signal difference. The concentration of the A domain, and pyrophosphatase could also be increased which would speed up the reaction and increase the level of ATP consumption.

\subsubsection{Synthesis of a novel indigoidine analogue}

Strong evidence was provided indicating that BpsA could synthesise a novel form of indigoidine from the glutamine analogue L-alanyl-L-glutamine, which I have named alaindigoidine. The compound had different solubility properties and had a maximal absorbance at a higher wavelength compared to indigoidine, and an increased retention time on a HPLC - $\mathrm{C}^{18}$ column was also different. Although not described in the main text, substantial time was spent attempting to use mass spectrometry to measure both indigoidine and ala-indigoidine. While other papers have successfully detected indigoidine using LC-MS (Gromek et al., 2016; Takahashi et al., 2007) we were hampered by needing to synthesise it in vitro, which resulted in substantially less 
indigoidine being formed compared to the in vivo synthesis and then purification method used in other studies. This small amount of indigoidine present coupled with the low level of solubility indigoidine possesses means that a reliable signal could not be detect for indigoidine. In future studies, it may be necessary to develop a large scale in vitro synthesis and then purification method to gain enough indigoidine and alaindigoidine to detect and quantify exactly what product is being synthesised.

The ability to generate ala-indigoidine could be useful for several different reasons, e.g. if the catalytic efficiency of the conversion of alanyl-glutamine into ala-indigoidine could be improved then it might form the basis for a novel biosensor to detect and quantify alanyl-glutamine in cell media. Currently, to the best of our knowledge, an enzymatic method to measure alanyl-glutamine is not available.

At least two other analogues of indigoidine have been identified in different bacterial strains. One has been purified from the deep-sea bacterium Shewanella violacea DSS12. This violet $3,3^{\prime}-$ bipyridyl pigment contains the same chromophore as indigoidine. The structure is an alkylated form of indigoidine having the chemical structure 5,5'didodecylamino-4,4'-dihydroxy-3,3'-diazadiphenoquinone-(2,2') (Fig. 6.14A) (Kobayashi et al., 2007). The authors noted in this paper that an indigoidine synthesising enzyme had not been identified in the $S$. violacea genome sequencing project which was completed in 2010. The genome sequence was published two years later (accession no: NC_014012.1) by Aono et al (2010), who did not report on a bioactive gene cluster capable of producing the indigoidine like pigment (Aono et al., 2010).

Using the protein BLAST tool on the NCBI site to probe the $S$. violacea genome with the A domain sequence of BpsA from S. lavendulae I identified a candidate biosynthetic gene (accession no: WP_013053246) that could be responsible for the alkylated form of indigoidine. Analysis using the NCBI conserved domain database shows WP_013053246 encodes a predicted hybrid NRPS-like protein with a modular structure of fatty acyl-Amp ligase-PCP domain-Reductase domain-A domain-PCP domain-TE domain. Exploring the function of WP_013053246 could represent a novel method for the attachment of a range of different prosthetic groups to indigoidine, potentially increasing the functionality of this structure. 
A second indigodine like compound was recently identified in the the cold aquatic enviroments of Andean Patagonia in Argentina (Day et al., 2017). This indigoidine like compound, synthesised by the the bacterium Vogesella sp. EB, was described by the researchers as cryoindigoidine (Fig. 6.14B) due to the researcher's hypothesis that it may help protect the bacterium against cryo-shock. The genome of the bacterium was also sequenced and a homolog to bpsA was identified along with several other genes, including an additional hypothetical NRPS protein that is not present in other indigoidine gene clusters and that is similar to the E. coli enterobactin synthase EntF (Day et al., 2017).

A

\section{B}

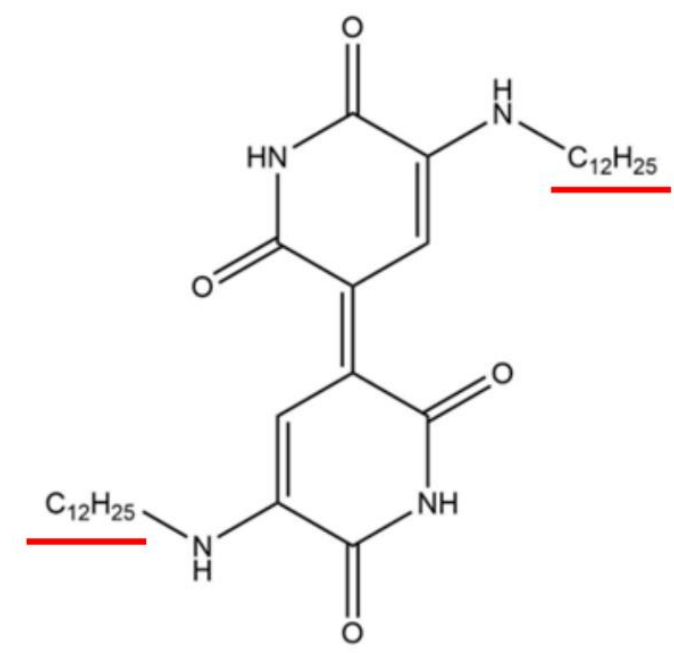<smiles>CC1=CC(=C2C=C(O)C(=O)NC2=O)C(=O)NC1=O</smiles>

Figure 6.14: Analogues of indigoidine identified in different bacterial strains: (A) The violet 3,3' - bipyridyl from Shewanella violacea DSS12 is an alkylated form of indigoidine, with a structure as determined by (Kobayashi et al., 2007). (B) An indigoidine analogue named "cryoindigoidine" from a species of Vogesella. Structure is adapted from (Day et al., 2017). The red bars represent the structural changes from indigoidine.

The identification of novel indigoidine compounds and the biosynthetic genes that produce them could be useful to guide the engineering of BpsA to synthesise forms of indigoidine having improved properties, e.g. as an industrial dye or redox sensor, or as a potential anti-microbial agent. The ability of associated genes or modules to modify indigoidine could also be investigated further as a method to modify the indigoidine scaffold. 
The A domain of BpsA appears to exercise a degree of flexibility in respect to the substrates that it recognises. There is a diverse range of commercially available glutamine analogues that have a diverse range of chemical structures. It might be commercially useful to be able to simply quantify several of these analogues including the cell culture additive glycyl-L-glutamine, which is a heat stable form of glutamine. Further investigation will be needed before we can confirm if novel analogues are definitely being synthesised from them.

\subsubsection{Investigation into the function of IgiF}

The purification of IgiF resulted in reasonable yields of soluble protein, which was somewhat unexpected given the complex quaternary structure of the protein. Different enzymes in the 4' oxalocrotonate tautomerase class have been purified in a variety of studies, with the majority of papers using either HPLC or a phenyl-sepharose column (Zandvoort et al., 2011) There is the potential that the 6His tags are interfering with the catalytic efficiency of the enzyme, resulting in a soluble but non-functional enzyme. One commercial supplier of enzymes does however provide 4' oxalocrotonate tautomerase from $B$. subtilis online as a $6 \mathrm{His}$ tagged protein (albeit at a price prohibitive to the current study), indicating that at least in that instance activity is not impaired. In future experiments, it might be beneficial to purify the $S$. lavendulae enzyme without a tag to confirm that it was not interfering with the function of the protein.

While the decision to use the $S$. lavendulae homologue was made, in case this precise variant was needed to interact effectively with BpsA, it could be the case that this enzyme does not in fact play this role in S. lavendulae. Consistent with this, it appears that the primary purpose of indigoidine in the bacterium is to protect it against oxidative stress rather than as an anti-microbial agent. Thus, IgiF from S. lavendulae might not possess any bioactivity. It could therefore be a worthwhile endeavour to synthesise the igiF described by Cude et al (2012).

The exact role of IgiF was never confirmed in Cude et al (2012) paper. In an effort to probe the antagonistic effects of the Phaeobacter species against Vibrio fischeri species, a transposon mutagenesis library was used to interrogate the relationship between indigoidine production and inhibition of $V$. fischeri on agar plates. Out of a library of 
6,048 mini-Tn5 transposon mutants, 45 were unable to inhibit $V$. fischeri. Several of the transposon inserts in these mutants were clustered in the indigoidine biosynthetic gene cluster. Interestingly, however none of the transposons were inserted into the igif gene (Cude et al., 2012). This could indicate that it is not important in the toxicity of indigoidine towards $V$. fischeri or that the relatively small size of the gene resulted in it, by chance, not being knocked out. It would be an important test to knock out igiF to determine if it was responsible for the conversion of indigoidine into the anti-microbial leuco-indigoidine form. We also cannot rule out the possibility that the conversion of indigoidine to lecuo-indigoidine might be non-enzymatically mediated or even whether leuco-indigoidine is responsible for the antimicrobial properties. 


\section{Chapter 7-Summary, conclusions and future directions}

\subsection{Research motivation}

Microorganisms are by far the most populous form of life on Earth. In nearly every environment on earth, bacteria have evolved a wealth of protein machinery in order to survive and prosper. One key mechanism that bacteria use to survive is the diversity of secondary metabolites they produce to give them a competitive advantage. Many of these secondary metabolites are produced by non-ribosomal peptide synthetases. These complex machines assemble bioactive peptides using modular assembly lines, which are capable of catalysing a range of chemical reactions.

The large and complex nature of NPRSs means that they can be difficult to study in vitro. This thesis focused on the development of novel bioassays using the single module NRPS BpsA. In addition to possessing a relatively simple domain structure, BpsA synthesises an easy to detect blue pigment called indigoidine from two molecules of L-glutamine in an ATP powered reaction. Indigoidine synthesis can be detected both in vivo and in vitro. The synthesis of indigoidine allows use of BpsA as a reporter for a variety of different conditions. Previous studies from the Ackerley lab have used BpsA in a functional screen for bioactive gene clusters, as a tool to characterise PPTases and probe PCP domain specificity. In addition, BpsA has been used as a model for PCP domain interactions. The work described in this thesis sought to expand on the uses already developed for BpsA and add additional tools to the BpsA toolkit.

Current antibiotics are increasingly losing effectiveness as bacteria evolve resistance to them. This means there is a crucial need for new drugs and drug targets to be developed. One potential drug target in bacteria is the PPTase super family. Previous work by Owen et al (2011) has shown that BpsA can be used to report on PPTase inhibition, and this study sought to optimise, expand and implement this assay for HTS. A primary focus was given to PPTases from $M$. tuberculosis and $P$. aeruginosa, as both of 
these pathogens represent a pressing health issue, with TB still having a large health footprint and $P$. aeruginosa increasingly becoming more resistant and difficult to treat. In addition to measuring the activity of PPTases and CP domains, this research looked at expanding the usefulness of BpsA as an in vitro biosensor for glutamine. This was further expanded into detection of ATP and the adenylation reaction. Finally, attention was given to the ability of BpsA to synthesis a variety of indigoidine analogues from different substrates, including the biotechnologically important alanyl-glutamine.

\subsection{Key findings}

\subsubsection{Development of an assay to detect PPTase inhibition}

Both PptT and PcpS can readily post-translationally modify BpsA. With a period of optimisation, it was possible to develop a HTS assay to detect the inhibition of these PPTases. It was then shown how the assay can be applied to commercially available drug libraries to facilitate the identification of PPTase inhibitors.

PptT is a relatively unstable PPTase which has low soluble yield when it is expressed in $E$. coli. This can be improved by the addition of $\mathrm{CoA}$ and $\mathrm{MgCl}_{2}$ during the lysis step. These co-factors increase the stability of PptT and allow it be used effectively in in vitro assays. After an optimisation campaign it was possible to detect the inhibition of PptT. This is an important step forward as other screens have focused on the prototypical PPTase Sfp, which is not from a major pathogen, and evidence has pointed towards PptT not binding the fluorescent CoA analogues that other published screens for Sfp have used. If this is the case then the use of native CoA in my screen should be a significant advantage. Chapter 3 describes the first published HTS screens for both PcpS and PptT.

Most PPTase inhibitors displayed generic Type II PPTase inhibition. Several novel inhibitors were reported for both PcpS and PptT, although in most cases these had higher $\mathrm{IC}_{50}$ values than the top inhibitors. The top PcpS inhibitor, $\mathrm{SCH}-202676$, was tested to see if it could inhibit $P$. aeruginosa growth. While very little anti-microbial activity was detected, a decrease in the secondary metabolite pyoverdine was apparent at the highest concentration tested $(250 \mu \mathrm{M})$. This suggested that while the PPTase 
inhibition did not have a substantial effect on the PA01 growth rate, there was nevertheless a reduction in PPTase activity. Monitoring pyoverdine production is a new and useful approach to detect the in vivo inhibition of PcpS, and confirms that only low levels of PPTase activity are required in vivo to support cell viability.

\subsubsection{Evolution of the PCP domain of BpsA to recognise Type I PPTases}

Previous work has shown the PCP domain of BpsA can be modified to recognise nonnative Type II PPTases (Owen et al., 2016). Work by Mofid et al and Finking et al had also shown that it was possible to modify the second alpha helix of PCP domains to be recognised by Type I PPTases. The research described in Chapter 4 combined these two approaches in order to try and develop a modified form of BpsA that was capable of being used as a reporter for Type I PPTases.

Two rational constructs were designed, employing the second alpha helices from $M$. tuberculosis AcpM and FAS-1 respectively. Testing showed that the substitution of the BpsA alpha helix had destroyed the recombinant enzymes' abilities to synthesise indigoidine when these were co-expressed with both PptT and EC-AcpS. A directed evolution campaign was then undertaken to improve the activity of the modified BpsA variants. Three pilot libraries were created based on wtBpsA, acpBpsA and facpBpsA. After one round of evolution, only the acpBpsA pilot library yielded variants capable of synthesising detectable levels of indigoidine in vivo. This is highly likely due to acpBpsA being a substrate of EC-AcpS. Two more rounds of evolution were then completed, resulting in mutants capable of producing detectable levels of indigoidine after 8-12 hours in vivo. This was a significant improvement over wtBpsA and acpBpsA, which were incapable of producing detectable indigoidine. Despite the mutants being activated by EC-AcpS, this result showed that directed evolution could be used to restore activity to a non-functional mutant.

The top mutants from each round were expressed and purified, and assayed for indigoidine production in vitro. The two top mutants from round three (RD3_Mut_11 and RD3_Mut_35) both displayed the characteristic indigoidine curve when assayed, without requiring in vitro activation by a PPTase. This confirmed they were being activated in vivo by EC-AcpS. The top mutants were then tested to see whether 
heightened levels of activity could be achieved following in vitro incubation with either TB-AcpS, PptT or EC-AcpS. None of the mutants synthesised increased levels of indigoidine when compared to the no PPTase controls. This suggested that nearcomplete conversion to the holo form had already occurred in vivo, and - if this was indeed the case - that the overall catalytic efficiency of the enzyme had been reduced substantially relative to wild type, despite the three rounds of directed evolution. Analysis of the amino acid sequence of the top mutants identified in the directed evolution campaign identified several residues as being key for gain of indigoidine synthesis. Interestingly two residue changes which occurred outside of the second alpha helix $(\mathrm{E} 11 \mathrm{~K}$ and $\mathrm{Q} 32 \mathrm{H})$ were also identified in a separate Ackerley lab directed evolution project that focused on evolving BpsA to recognise Type II PPTases after an second alpha helix substitution event. It may be that advance rational alteration of these two residues when modifying the second alpha helix of the PCP domain could reduce the number mutational changes needed to improve activity, and thereby help develop a generic PCP domain scaffold for recognising second alpha helix substitutions. Several other important mutations were identified, for example all the mutants that were sequenced from the second and third round of evolution contained the T52I substitution indicating that at this position an isoleucine (or other hydrophobic residue) may be critical for functionality. This will be an important consideration if another directed evolution campaign is attempted in the future.

\subsubsection{Development of a biosensor to quantify L-glutamine}

The development of an assay to quantify glutamine is an important milestone in the adaption of NPRSs for different biotechnological functions, as most NRPS research has either been focused on the fundamental understanding of NRPS function or attempting engineer them to synthesise different products. By taking advantage of the direct ratio between the amount of indigoidine synthesised and the amount of glutamine present in a test sample, I was able to develop an assay that was able to accurately quantify glutamine levels.

A key issue with using BpsA is that it needs to be expressed in its inactive apo state. By developing a protocol to post-translationally modify BpsA to the active holo state prior 
to purification, I was able to purify active BpsA in high yields. This method also took advantage of the endogenous CoA present in bacterial cells, which helped to reduce the amount of CoA required and means that holo-BpsA could readily be purified on a commercial scale.

Initial attempts to measure glutamine concentrations using either the velocity of the reaction or the peak indigoidine absorbance achieved during synthesis proved inaccurate due to indigoidine being insoluble in water and dropping out of solution. Instead, a two-step method was developed: First, the indigoidine accrued until all of the glutamine in a sample had been consumed; and then DMSO was added to resolubilise the indigoidine and an end-point measurement was taken. The DMSO stop method provided a simple, accurate and reproducible way to quantify L-glutamine in a range of biologically and commercially relevant conditions. One exceptional circumstance was encountered when I initially tried to resolubilise indigoidine synthesised from glutamine in blood plasma sample. The DMSO and proteins present in the plasma interacted with each other causing signal interference. This was remedied by deproteinating the plasma, after which accurate quantification of glutamine in plasma samples was easily achievable. The commercial applicability of this assay is illustrated by the patent and support VicLink (the commercialisation arm of VUW) has provided to support development of this work.

\subsubsection{Additional uses of BpsA}

After the success with the L-glutamine sensor I applied the same methodology to detect ATP. BpsA proved capable of measuring ATP concentrations in a range of samples. While there are more accurate methods for the detection of ATP already available, this system may be useful, e.g. if a component of the commercially available colourimetric assays is incompatible with the sample being measured. I also showed that this system could readily be adapted for the detection of adenylation by a purified NRPS A domain. By measuring the consumption of ATP, it was possible to detect the substrate specificity of the A domain from EntF. This method provides an alternative method for measuring adenylation which could be used if traditional substrate identification assays such as the malachite green assay are unavailable. 
The ability of BpsA to synthesise indigoidine-like compounds from glutamine analogues was also explored. It was shown that BpsA can synthesise a coloured compound from the glutamine analogue alanyl-glutamine, yielding a product I have called alaindigoidine. Synthesis of this product occurs much more slowly than wildtype indigoidine. Ala-indigoidine appears to be more soluble than indigoidine in water and has a longer retention time on an HPLC column, providing strong evidence that a novel compound is indeed being synthesised. Alanyl-glutamine is an important cell culture additive and it would be useful to develop a biosensor to detect it. This research shows that BpsA has at least low-level ability to synthesise ala-indigoidine from alanylglutamine and, with further development, this could form the basis of a biosensor.

\subsection{Critical evaluation of this work}

\subsubsection{Development of HTS to assay to detect PPTase inhibition}

The PPTase inhibitor screen was used to successfully detect novel inhibitors of both PcpS and PptT. While BpsA can be used to interrogate the kinetic properties of PPTases effectively, it does have some drawbacks as a HTS tool. The biggest issues are that it is limited to 96 well plates, and due to the assay set up it requires the manual dispensing of reagents. This means that while it can be used to screen commercial drug libraries such as LOPAC ${ }^{1280}$ and the NIH Diversity Set II, it ca not be expanded into the same level of HTS screening as the Burkart Laboratory achieved with their Sfp FP assay, in their screen of over 300,000 compounds (Foley et al., 2010). In addition to this, the purification of PptT in the presence of exogenous CoA may have reduced the sensitivity of the assay, with previous work by Owen et al having shown that increasing CoA concentrations also increased the $I_{50}$ of various inhibitors (Owen et al., 2011). This, coupled with the increased PptT concentration required relative to PcpS, could have been a reason for fewer inhibitors being identified. For example, in screening the $\mathrm{NIH}$ Diversity Set II, far fewer PptT inhibitors were detected compared to PcpS.

The pilot in vivo study of SCH-202676 showed that PcpS inhibition could potentially be detected in vivo, however this proof-of-principle study did not result in a substantial antimicrobial effect. Part of the reason for this could be due to the competent efflux 
system that $P$. aeruginosa possesses. It could be that $\mathrm{SCH}-202676$ can potentially inhibit the growth of $P$. aeruginosa but it is just being pumped out of the cell before it has an effect. Further work needs to be undertaken to determine whether this is the case, for example using a strain of $P$. aeruginosa with RND efflux systems (Poole, 2001) knocked out, or assessing the effect of $\mathrm{SCH}-202676$ being co-administered with the efflux inhibitor phenylalanine-arginine beta-naphthylamide (Pages et al., 2005).

\subsubsection{Evolution of the PCP domain of BpsA to accept type I PPTases}

Critical evaluation of this work was covered in detail in Chapter four.

\subsubsection{Development of a biosensor to quantify L-glutamine}

The BpsA glutamine biosensor is a commercially viable assay that is comparable to or better than current colourimetric assays on the market sold by leading companies such as Sigma Aldrich. In some situations however this assay is not suitable. For example, when assaying blood, the DMSO in the assay reacts with proteins or other components (although this issue is surmountable by using a cut off column to deproteinate the serum). Moreover, it is possible that some solutions may render the assay unusable, e.g. if there is a metal chelator such as EDTA present, or a strong reactive species that may degrade the indigoidine.

\subsubsection{Development of a biosensor to quantify ATP}

While I demonstrated that the BpsA biosensor is accurate across a concentration of 20 $\mu \mathrm{M}$ to $2000 \mu \mathrm{M}$ ATP, it is significantly less sensitive than current detection methods such as the two-step oxidation of luciferin which can detect ATP concentrations down to the attomolar range. It is also less sensitive than other colourimetric kits currently available such as the ATP Colorimetric Assay Kit produced by Sigma Aldrich ${ }^{\circledR}$, which has a stated detection limit of $2 \mu \mathrm{M}$. However, if the anticipated ATP concentration is within our detection range, our method provides an alternative that may be faster and more economical.

The novel method for the detection of NRPS-mediated substrate adenylation that was developed here, is unlikely to be useful for anyone outside of a laboratory that already 
works with BpsA or a homologue thereof. There are also other potential limitations. The most significant issue is that the assay relies on a negative result for detection, which means that false positives are much more likely. For example, not adding a reagent into the assay would give the same result as an A domain consuming all of the ATP during adenylation. This issue is surmountable with good experimental design and ensuring that adequate positive controls are used.

The assay is less accurate than the standard malachite green assay. It is difficult to more precisely determine the minimal detection range as an A domain with low levels of activity will consume less ATP than an A domain with high levels of activity. Despite these drawbacks, the method does provide a new method for the detection of adenylation that could provide useful under certain circumstances.

\subsubsection{Synthesis of indigoidine analogues}

BpsA appears to be capable of the synthesis of indigoidine analogues, with alaindigoidine synthesised from alanyl-glutamine having a different absorbance spectrum and a different HPLC retention time to regular indigoidine. However, we were unable to confirm the exact makeup of the indigoidine analogue due to being unable to detect it using LC-MS. Other papers that have detected indigoidine have used a large amount of purified compound and an in vivo method for preparation of this compound (Cude et al., 2012; Gromek et al., 2016; Takahashi et al., 2007). This method was not available for us as endogenous cellular L-glutamine would easily outcompete the alanyl-glutamine and give rise to high levels of contaminating indigoidine product.

\subsection{Future directions}

\subsubsection{In vitro detection of PPTase inhibition}

The modified screening protocol discussed in Section 3.7 should allow more rapid screening for inhibitors of PptT and PcpS. It may also allow the miniaturisation of the assay so that it can be performed in 384 well plates. This will reduce the amount of enzyme and drug necessary to detect inhibition of PPTases. It should now be possible to use liquid handling robots, as available in the Victoria University Chemical Genetics 
Laboratory, to conduct all steps in the assay. This would greatly facilitate the screening of larger chemical libraries. In addition to the two PPTases that were screened, it should be possible to adapt the assay for the detection of a range of different PPTases that could be of interest, including fungal PPTases. It has been shown that several fungal species appear to be substantially more sensitive than $P$. aeruginosa to PPTase inhibitors (Johns et al., 2017).

Several compounds that were identified as inhibitors also have analogues available from commercial suppliers and the NIH Developmental Therapeutics Programme. These analogues are worth exploring and could have improved pharmokinetic activities or inhibitory activity. Our collaborators at the University of Auckland have successfully crystallised PptT (Jung et al., 2014). If novel inhibitors are identified it may be possible to conduct structure activity relationship studies and develop new PptT inhibitors guided by this structural information.

\subsubsection{In vivo PPTase inhibition}

Despite the strong efflux systems that are characteristic of Gram-negative bacteria, PcpS may be a better antibiotic target that other type II PPTases such as Sfp or PptT, due to it being the sole PPTase in P. aeruginosa. Unlike PptT no real characterisation of PcpS as a potential drug target has been carried out other than it being essential. Before further work is completed it will be important to characterise the effect of PcpS inhibition on the growth of $P$. aeruginosa. The Ackerley lab has a strain of $P$. aeruginosa that has PcpS knocked out and complemented by a chromosomally integrated version of E. coli acpS. This strain is incapable of growing on iron limited media. Overexpression and knockdown of PcpS in this strain, with concomitant monitoring of pyoverdine production levels, may help to characterise the level of inhibition required to be bactericidal. Foley et al found that the main resistance mechanism for ML-267 was efflux. In vivo antimicrobial studies with efflux inhibitors such as $\mathrm{Pa} \beta \mathrm{N}$ or using efflux pump knockout strains may yield improved results for initial testing of PcpS inhibitors

PptT inhibitors could also be tested in M. smegmatis, as the PptT homolog has also been shown to be essential (Chalut et al., 2006). Thus PptT inhibitors might reasonably be tested for in vivo activity against the faster growing $M$. smegmatis strain. 
Additionally, M. smegmatis could be transformed with the bpsA gene, which might then act as a reporter for in vivo PPTase inhibition.

\subsubsection{Evolution of the BpsA PCP domain for activity with type I PPTases}

An alternative approach could also be attempted to the evolution method described in Chapter Four to generate improved helix-substituted variants. Previous work by Zhou et al and has shown that phage display is an effective method for improving the recognition between PPTases and the second alpha helix in CP domains (Zhou et al., 2007). Modified forms of the BPsA PCP domain could be designed with minimal changes to the native amino acid sequence and then selected for using phage display. This would allow the screening of much larger libraries, as it could be done without the need for a functional selection step. Once several templates had been enriched for they could then be cloned into the BpsA swapping vector and directed evolution could be used to improve catalytic function of the PCP domain. This method would also hopefully remove the interference with E. coli AcpS.

\subsubsection{Synthesis of indigoidine analogues}

Indigoidine and indigoidine like compounds appear to have a range of uses in nature, including as an antioxidant, a cryoprotectant and as an anti-microbial compound. Metagenomic analysis and mining may uncover novel forms of indigoidine having useful biotechnological properties. Blast searches through published genomes might provide a means to unearth modified forms of BpsA or tailoring enzymes within the bioactive cluster that promote the synthesis of various useful indigoidine analogues.

The exact mechanism of indigoidine synthesis and how its antimicrobial properties function is unknown. Several genes in the bioactive clusters that govern synthesis of indigoidine have an unknown function. It would be interesting to express a range of these genes within E. coli and try and determine their overall function. For example, taking the entire bioactive gene cluster from Roseobacterium and try and determine what genes confer the antimicrobial properties to indigoidine. 


\subsubsection{Engineering BpsA for novel uses}

The (relatively) simple single module nature of BpsA means that it is an ideal candidate for engineering efforts to probe NRPS function and develop new biochemical assays.

\subsubsection{Engineering the A domain}

Several different modifications could be made to the A domain in order to facilitate a range of different reactions. For example, in this work multiple analogues of glutamine were tested for activity with BpsA, and alanyl-glutamine produced detectable pigment. By engineering the $A$ domain, it may be possible to improve the activity between BpsA and analogues such as alanyl-glutamine. A different directed evolution strategy would have to be attempted however, as for in vivo evolution endogenous cellular glutamine would out-compete alanyl glutamine. Cell free synthesis (Carlson et al., 2012) could potentially be explored or the A domain could be tested in isolation using an assay that measures adenylation.

Though not discussed in this thesis, considerable time was spent trying to develop a modified form of BpsA capable of synthesising indigoidine from D-glutamine. This ultimately proved to be unsuccessful, potentially due to an incomplete range of residues targeted for mutagenesis. By changing the residues selected for mutagenesis it might be possible to develop a variant of BpsA capable of synthesising indigoidine from Dglutamine. This would be a useful step forward for two reasons. Firstly, it would represent the first time an $A$ domain had been engineered to accept the $D$ form of an amino acid; and secondly it could act as a reporter for D-glutamine synthesis. This form of BpsA then could be used as a reporter for evolving racemases and epimerisation domains to be able to have improved activity in the conversion of $L$ to $D$ glutamine, $a$ commercially expensive molecule.

\subsubsection{Engineering the oxidation domain}

The BpsA oxidation domain also has not been studied in great detail. Work by Takahashi et al showed that it could be inactivated by the modification of key residues within the domain which stopped it being able to bind FMN. Intriguingly, they mentioned in the paper that BpsA with a non-functional oxidation domain (-oxBpsA) could have its 
functionality restored by the co-expression of the BpsA oxidation domain in trans. This might provide a means to probe oxidation domain function using BpsA.

An initial experiment would be necessary, but if -oxBpsA had functionality restored by oxidation domains from different NRPSs then this could provide a novel method to screen metagenomic libraries for oxidation domains and enzymes capable of catalysing the oxidation of the indigoidine intermediates. That is, metagenomic fragments could be co-expressed with -oxBpsA and an activating PPTase, and if the colonies turned blue they would likely have gained a novel oxidation domain. Additionally, not much is known about the substrate specificity of NRPS oxidation domains. BpsA could be used as a reporter for assessing oxidation domain specificity.

\subsubsection{Engineering the TE domain}

Some papers have shown that TE domains or modified $\mathrm{C}$ domain are mobile and that these can be functional in situ (Cai et al., 2017). BpsA could be modified to have the TE domain removed and then used to screen for TE domains in metagenomes or other different functions.

\subsection{Concluding remarks}

BpsA has been a workhorse in our laboratory. Its flexible and elegant nature means that it can be adapted for a diverse range of uses. In previous studies it has been used to probe PPTase function and CP interactions, mine metagenomic libraries for promising new bioactive gene clusters and probe domain function and interactions. BpsAs simplistic, single module structure and the relative ease in which it can be manipulated in vivo and in vitro has led to a range of novel uses being developed. This thesis has used BpsA as a reporter for PPTase inhibition, PCP domain function and the accurate detection and quantification of glutamine and ATP. BpsA was also used to synthesise a novel analogue of indigoidine. I hope that in the future more "blue skies" research is undertaken as it seems like new uses for it are yet to be discovered. 


\section{Bibliography}

Acker, M.G., and Auld, D.S. (2014). Considerations for the design and reporting of enzyme assays in high-throughput screening applications. Perspectives in Science 1, 5673.

Ackerley, D. F. (2016). Cracking the non-ribosomal code. Cell Chemical Biology 23, 535537.

Ackerley, D. F., Brown, A. S., \& Robins, K. J. (2015). Methods of detecting and measuring glutamine and analogues thereof, and methods related thereto. PCT patent filing WO/2015/084189, published $11^{\text {th }}$ June 2015 .

Agostini, F., and Biolo, G. (2010). Effect of physical activity on glutamine metabolism. Current Opinion in Clinical Nutrition and Metabolic care 13, 58-64.

Alberts, A.W., and Vagelos, P.R. (1966). Studies of acyl carrier protein and coenzyme A in Escherichia coli pantothenate or betaalanine auxotrophs. The Journal of Biological Chemistry 241, 5201-5204.

Ansari, M.Z., Yadav, G., Gokhale, R.S., and Mohanty, D. (2004). NRPS-PKS: a knowledgebased resource for analysis of NRPS/PKS megasynthases. Nucleic Acids Research 32, W405-W413.

Aono, E., Baba, T., Ara, T., Nishi, T., Nakamichi, T., Inamoto, E., Toyonaga, H., Hasegawa, M., Takai, Y., Okumura, Y., et al. (2010). Complete genome sequence and comparative analysis of Shewanella violacea, a psychrophilic and piezophilic bacterium from deep sea floor sediments. Molecular BioSystems 6, 1216-1226.

Arakawa, T., Ejima, D., Tsumoto, K., Obeyama, N., Tanaka, Y., Kita, Y., and Timasheff, S.N. (2007). Suppression of protein interactions by arginine: a proposed mechanism of the arginine effects. Biophysical Chemistry 127, 1-8.

Baell, J.B., and Holloway, G.A. (2010). New substructure filters for removal of pan assay interference compounds (PAINS) from screening libraries and for their exclusion in bioassays. Journal of Medicinal Chemistry 53, 2719-2740. 
Baltz, R.H. (2010). Streptomyces and Saccharopolyspora hosts for heterologous expression of secondary metabolite gene clusters. Journal of Industrial Microbiology \& Biotechnology 37, 759-772.

Barekzi, N., Joshi, S., Irwin, S., Ontl, T., and Schweizer, H.P. (2004). Genetic characterization of $p c p S$, encoding the multifunctional phosphopantetheinyl transferase of Pseudomonas aeruginosa. Microbiology 150, 795-803.

Beer, R., Herbst, K., Ignatiadis, N., Kats, I., Adlung, L., Meyer, H., Niopek, D., Christiansen, T., Georgi, F., Kurzawa, N., et al. (2014). Creating functional engineered variants of the single-module non-ribosomal peptide synthetase IndC by T domain exchange. Molecular BioSystems 10, 1709-1718.

Beld, J., Sonnenschein, E.C., Vickery, C.R., Noel, J.P., and Burkart, M.D. (2014). The phosphopantetheinyl transferases: catalysis of a post-translational modification crucial for life. Natural Product Reports 31, 61-108.

Belshaw, P.J., Walsh, C.T., and Stachelhaus, T. (1999). Aminoacyl-CoAs as probes of condensation domain selectivity in nonribosomal peptide synthesis. Science $284,486-$ 489.

Bitok, J.K., Lemetre, C., Ternei, M.A., and Brady, S.F. (2017). Identification of biosynthetic gene clusters from metagenomic libraries using PPTase complementation in a Streptomyces host. FEMS Microbiology Letters 364.

Bloudoff, K., and Schmeing, T.M. (2017). Structural and functional aspects of the nonribosomal peptide synthetase condensation domain superfamily: discovery, dissection and diversity. Biochimica et Biophysica Acta (BBA) - Proteins and Proteomics. Bouatra, S., Aziat, F., Mandal, R., Guo, A.C., Wilson, M.R., Knox, C., Bjorndahl, T.C., Krishnamurthy, R., Saleem, F., Liu, P., et al. (2013). The Human Urine Metabolome. PLOS ONE 8, e73076.

Brinster, S., Lamberet, G., Staels, B., Trieu-Cuot, P., Gruss, A., and Poyart, C. (2009). Type II fatty acid synthesis is not a suitable antibiotic target for Gram-positive pathogens. Nature 458, 83-86. 
Brown, A.S., Robins, K.J., and Ackerley, D.F. (2017). A sensitive single-enzyme assay system using the non-ribosomal peptide synthetase BpsA for measurement of Lglutamine in biological samples. Scientific Reports 7, 41745.

Bruner, S.D., Weber, T., Kohli, R.M., Schwarzer, D., Marahiel, M.A., Walsh, C.T., and Stubbs, M.T. (2002). Structural basis for the cyclization of the lipopeptide antibiotic surfactin by the thioesterase Domain SrfTE. Structure 10, 301-310.

Bunkoczi, G., Pasta, S., Joshi, A., Wu, X., Kavanagh, K.L., Smith, S., and Oppermann, U. (2007). Mechanism and substrate recognition of human holo ACP synthase. Chemical Biology 14, 1243-1253.

Caboche, S., Leclère, V., Pupin, M., Kucherov, G., and Jacques, P. (2010). Diversity of Monomers in non-ribosomal peptides: towards the prediction of origin and biological activity. Journal of Bacteriology 192, 5143-5150.

Cai, X., Nowak, S., Wesche, F., Bischoff, I., Kaiser, M., Fürst, R., and Bode, H.B. (2017). Entomopathogenic bacteria use multiple mechanisms for bioactive peptide library design. Nature Chemistry 9, 379-386.

Carlson, E.D., Gan, R., Hodgman, C.E., and Jewett, M.C. (2012). Cell-Free protein synthesis: Applications come of age. Biotechnology Advances 30, 1185-1194.

Chalut, C., Botella, L., de Sousa-D’Auria, C., Houssin, C., and Guilhot, C. (2006). The nonredundant roles of two 4'-phosphopantetheinyl transferases in vital processes of Mycobacteria. Proceedings of the National Academy of Sciences 103, 8511-8516.

Charlop-Powers, Z., Banik, J.J., Owen, J.G., Craig, J.W., and Brady, S.F. (2013). Selective enrichment of environmental DNA libraries for genes encoding non-ribosomal peptides and polyketides by phosphopantetheine transferase-dependent complementation of siderophore biosynthesis. ACS Chemical Biology 8, 138-143.

Chatterjee, J., Rechenmacher, F., and Kessler, H. (2013). N-methylation of peptides and proteins: an important element for modulating biological functions. Angewandte Chemie International Edition 52, 254-269. 
Chen, J., and Herrup, K. (2012). Glutamine acts as a neuroprotectant against DNA damage, beta-amyloid and $\mathrm{H}_{2} \mathrm{O}_{2}$-induced stress. PLOS ONE 7, e33177.

Cheng, Y., Liao, L., Chang, C., Zhou, J., Deng, Y., and Zhang, L.-H. (2016). A Sfp-type phosphopantetheinyl transferase $\mathrm{ZmsO}$ is essential for zeamines production and the virulence of Dickeya zeae. European Journal of Plant Pathology 146, 937-948.

Chu, M., Mierzwa, R., Xu, L., Yang, S.-W., He, L., Patel, M., Stafford, J., Macinga, D., Black, T., Chan, T.-M., et al. (2003). Structure elucidation of SCH 538415, a novel acyl carrier protein synthase inhibitor from a microorganism. Bioorganic \& Medicinal Chemistry Letters 13, 3827-3829.

Chuang, A.J., Killam, K.F., Chuang, R.Y., Rice, W.G., Schaeffer, C.A., Mendeleyev, J., and Kun, E. (1993). Inhibition of the replication of native and 3'-azido-2', $3^{\prime}$-dideoxythymidine (AZT)-resistant simian immunodeficiency virus (SIV) by 3-nitrosobenzamide. FEBS Letters 326, 140-144.

Cole, S.T., Brosch, R., Parkhill, J., Garnier, T., Churcher, C., Harris, D., Gordon, S.V., Eiglmeier, K., Gas, S., Barry, C.E., et al. (1998). Deciphering the biology of Mycobacterium tuberculosis from the complete genome sequence. Nature 393, 537-544.

Conti, E., Stachelhaus, T., Marahiel, M.A., and Brick, P. (1997). Structural basis for the activation of phenylalanine in the non-ribosomal biosynthesis of gramicidin S. The EMBO Journal 16, 4174-4183.

Costa, P.D., Andrade, N.J., Brandão, S.C.C., Passos, F.J.V., and Soares, N.d.F.F. (2006). ATP-bioluminescence assay as an alternative for hygiene-monitoring procedures of stainless steel milk contact surfaces. Brazilian Journal of Microbiology 37, 345-349.

Cude, W.N., Mooney, J., Tavanaei, A.A., Hadden, M.K., Frank, A.M., Gulvik, C.A., May, A.L., and Buchan, A. (2012). Production of the Antimicrobial secondary metabolite indigoidine contributes to competitive surface colonization by the marine Roseobacter Phaeobacter sp. Strain Y4I. Applied and Environmental Microbiology 78, 4771-4780.

Day, P.A., Villalba, M.S., Herrero, O.M., Arancibia, L.A., and Alvarez, H.M. (2017). Formation of indigoidine derived-pigments contributes to the adaptation of Vogesella 
sp. strain EB to cold aquatic iron-oxidizing environments. Antonie van Leeuwenhoek 110, 415-428.

De Crecy-Lagard, V., Marliere, P., and Saurin, W. (1995). Multienzymatic non ribosomal peptide biosynthesis: identification of the functional domains catalysing peptide elongation and epimerisation. Comptes rendus de I'Academie des sciences Serie III, Sciences de la vie 318, 927-936.

Déchelotte, P., Hasselmann, M., Cynober, L., Allaouchiche, B., Coëffier, M., Hecketsweiler, B., Merle, V., Mazerolles, M., Samba, D., Guillou, Y.M., et al. (2006). Lalanyl-L-glutamine dipeptide-supplemented total parenteral nutrition reduces infectious complications and glucose intolerance in critically ill patients: The French controlled, randomized, double-blind, multicenter study. Critical Care Medicine 34, 598-604.

Downham, A., and Collins, P. (2000). Colouring our foods in the last and next millennium. International Journal of Food Science \& Technology 35, 5-22.

Doyle, C., and Butler, M. (1990). The effect of $\mathrm{pH}$ on the toxicity of ammonia to a murine hybridoma. Journal of Biotechnology 15, 91-100.

Drake, E.J., Miller, B.R., Shi, C., Tarrasch, J.T., Sundlov, J.A., Allen, C.L., Skiniotis, G., Aldrich, C.C., and Gulick, A.M. (2016). Structures of two distinct conformations of holonon-ribosomal peptide synthetases. Nature 529, 235-238.

Du, L., Chen, M., Sánchez, C., and Shen, B. (2000). An oxidation domain in the Blmlll nonribosomal peptide synthetase probably catalyzing thiazole formation in the biosynthesis of the anti-tumor drug bleomycin in Streptomyces verticillus ATCC15003. FEMS Microbiology Letters 189, 171-175.

Duckworth, B.P., and Aldrich, C.C. (2010). Development of a high-throughput fluorescence polarization assay for the discovery of phosphopantetheinyl transferase inhibitors. Analytical Biochemistry 403, 13-19.

Dym, O., Albeck, S., Peleg, Y., Schwarz, A., Shakked, Z., Burstein, Y., and Zimhony, O. (2009). Structure-function analysis of the acyl carrier protein synthase (AcpS) from Mycobacterium tuberculosis. Journal of Molecular Biology 393, 937-950. 
Eagle, H., Oyama, V.I., Levy, M., Horton, C.L., and Fleischman, R. (1956). The growth response of mammalian cells in tissue culture to L-glutamine and L-glutamic acid. The Journal of Biological Chemistry 218, 607-616.

Elazari-Volcani, B. (1939). On Pseudomonas indigofera (Voges) Migula and its pigment. Archiv für Mikrobiologie 10, 343-358.

Fernandes, N.D., and Kolattukudy, P.E. (1996). Cloning, sequencing and characterization of a fatty acid synthase-encoding gene from Mycobacterium tuberculosis var. bovis BCG. Gene 170, 95-99.

Finking, R., and Marahiel, M.A. (2004). Biosynthesis of non-ribosomal Peptides. Annual Review of Microbiology 58, 453-488.

Finking, R., Mofid, M.R., and Marahiel, M.A. (2004). Mutational analysis of peptidyl carrier protein and acyl carrier protein synthase unveils residues involved in protein-protein recognition. Biochemistry 43, 8946-8956.

Foley, T.L., Rai, G., Yasgar, A., Attene-Ramos, M.S., Burkart, M.D., Simeonov, A., Jadhav, A., and Maloney, D.J. (2010). Discovery of ML 267 as a Novel inhibitor of pathogenic Sfp phosphopantetheinyl transferase (PPTase). In Probe Reports from the NIH Molecular Libraries Program (Bethesda (MD): National Center for Biotechnology Information (US)).

Foley, T.L., Rai, G., Yasgar, A., Daniel, T., Baker, H.L., Attene-Ramos, M., Kosa, N.M., Leister, W., Burkart, M.D., Jadhav, A., et al. (2014). 4-(3-Chloro-5(trifluoromethyl)pyridin-2-yl)-N-(4-methoxypyridin-2-yl)piperazine-1-carbothioamide (ML267), a Potent inhibitor of bacterial phosphopantetheinyl transferase that attenuates secondary metabolism and thwarts bacterial growth. Journal of Medicinal Chemistry 57, 1063-1078.

Foley, T.L., Young, B.S., and Burkart, M.D. (2009). Phosphopantetheinyl transferase inhibition and secondary metabolism. FEBS Journal 276, 7134-7145.

Gehring, A.M., Lambalot, R.H., Vogel, K.W., Drueckhammer, D.G., and Walsh, C.T. (1997). Ability of Streptomyces spp. aryl carrier proteins and coenzyme A analogs to serve as substrates in vitro for E. coli holo-ACP synthase. Chemistry \& Biology 4, 17-24. 
Gewolb, J. (2002). Working outside the protein-synthesis rules. Science 295, 2205-2207.

Gilbert, A.M., Kirisits, M., Toy, P., Nunn, D.S., Failli, A., Dushin, E.G., Novikova, E., Petersen, P.J., Joseph-McCarthy, D., McFadyen, I., et al. (2004). Anthranilate 4H-oxazol5-ones: novel small molecule antibacterial acyl carrier protein synthase (AcpS) inhibitors. Bioorganic \& Medicinal Chemistry Letters 14, 37-41.

Gokulan, K., Aggarwal, A., Shipman, L., Besra, G.S., and Sacchettini, J.C. (2011). Mycobacterium tuberculosis acyl carrier protein synthase adopts two different $\mathrm{pH}$ dependent structural conformations. Acta Crystallographica Section D 67, 657-669. Gromek, S.M., Suria, A.M., Fullmer, M.S., Garcia, J.L., Gogarten, J.P., Nyholm, S.V., and Balunas, M.J. (2016). Leisingera sp. JC1, a bacterial Isolate from hawaiian bobtail squid eggs, produces indigoidine and differentially inhibits Vibrios. Frontiers in Microbiology 7, 1342.

Gulick, A.M. (2009). Conformational dynamics in the Acyl-CoA synthetases, adenylation domains of non-ribosomal peptide synthetases, and firefly luciferase. ACS Chemical Biology 4, 811-827.

Haslinger, K., Peschke, M., Brieke, C., Maximowitsch, E., and Cryle, M.J. (2015). X-domain of peptide synthetases recruits oxygenases crucial for glycopeptide biosynthesis. Nature 521, 105-109.

Haslinger, K., Redfield, C., and Cryle, M.J. (2015). Structure of the terminal PCP domain of the non-ribosomal peptide synthetase in teicoplanin biosynthesis. Proteins: Structure, Function, and Bioinformatics 83, 711-721.

Hoffmann, K., Schneider-Scherzer, E., Kleinkauf, H., and Zocher, R. (1994). Purification and characterization of eukaryotic alanine racemase acting as key enzyme in cyclosporin biosynthesis. The Journal of Biological Chemistry 269, 12710-12714.

Hoppert, M., Gentzsch, C., and Schörgendorfer, K. (2001). Structure and localization of cyclosporin synthetase, the key enzyme of cyclosporin biosynthesis in Tolypocladium inflatum. Archives of Microbiology 176, 285-293. 
Hur, G.H., Vickery, C.R., and Burkart, M.D. (2012). Explorations of catalytic domains in non-ribosomal peptide synthetase enzymology. Natural Product Reports 29, 1074-1098.

Ikeda, A., Nishiumi, S., Shinohara, M., Yoshie, T., Hatano, N., Okuno, T., Bamba, T., Fukusaki, E., Takenawa, T., Azuma, T., et al. (2012). Serum metabolomics as a novel diagnostic approach for gastrointestinal cancer. Biomedical Chromatography: BMC 26, 548-558.

Imamoto, Y., Tanaka, H., Takahashi, K., Konno, Y., and Suzawa, T. (2013). Advantages of AlaGln as an additive to cell culture medium: use with anti-CD20 chimeric antibodyproducing POTELLIGENT ${ }^{\mathrm{TM}}$ CHO cell lines. Cytotechnology 65, 135-143.

Johansson, P., Mulinacci, B., Koestler, C., Vollrath, R., Oesterhelt, D., and Grininger, M. (2009). multimeric options for the auto-activation of the Saccharomyces cerevisiae FAS Type I Megasynthase. Structure 17, 1063-1074.

Johns, A., Scharf, D.H., Gsaller, F., Schmidt, H., Heinekamp, T., Straßburger, M., Oliver, J.D., Birch, M., Beckmann, N., Dobb, K.S., et al. (2017). A nonredundant phosphopantetheinyl transferase, PptA, Is a novel antifungal target that directs secondary metabolite, siderophore, and lysine biosynthesis in Aspergillus fumigatus and is critical for pathogenicity. mBio 8.

Joseph-McCarthy, D., Parris, K., Huang, A., Failli, A., Quagliato, D., Dushin, E.G., Novikova, E., Severina, E., Tuckman, M., Petersen, P.J., et al. (2005). Use of structure-based drug design approaches to obtain novel anthranilic acid acyl carrier protein synthase inhibitors. Journal of Medicinal Chemistry 48, 7960-7969.

Jung, J., Bashiri, G., Johnston, J.M., Brown, A.S., Ackerley, D.F., and Baker, E.N. (2014). Crystal structure of the essential Mycobacterium tuberculosis phosphopantetheinyl transferase PptT, solved as a fusion protein with maltose binding protein. Journal of Structural Biology 188, 274-278.

Keating, T.A., Ehmann, D.E., Kohli, R.M., Marshall, C.G., Trauger, J.W., and Walsh, C.T. (2001). Chain termination steps in nonribosomal peptide synthetase assembly lines: directed acyl-S-enzyme breakdown in antibiotic and siderophore biosynthesis. Chembiochem: a European Journal of Chemical Biology 2, 99-107. 
Keating, T.A., Marshall, C.G., Walsh, C.T., and Keating, A.E. (2002). The structure of VibH represents non-ribosomal peptide synthetase condensation, cyclization and epimerization domains. Nature Structural Biology 9, 522-526.

Kerr, K.G., and Snelling, A.M. (2009). Pseudomonas aeruginosa: a formidable and everpresent adversary. Journal of Hospital Infection 73, 338-344.

Kimmich, G.A., Randles, J., and Brand, J.S. (1975). Assay of picomole amounts of ATP, ADP, and AMP using the luciferase enzyme system. Analytical Biochemistry 69, 187-206.

Kittilä, T., Mollo, A., Charkoudian, L.K., and Cryle, M.J. (2016). New structural data reveal the motion of carrier proteins in non-ribosomal peptide synthesis. Angewandte Chemie (International Ed in English) 55, 9834-9840.

Kobayashi, H., Nogi, Y., and Horikoshi, K. (2007). New violet 3,3'-bipyridyl pigment purified from deep-sea microorganism Shewanella violacea DSS12. Extremophiles : Life Under Extreme Conditions 11, 245-250.

Konz, D., and Marahiel, M.A. (1999). How do peptide synthetases generate structural diversity? Chemistry \& Biology 6, R39-R48.

Kosa, N.M., Foley, T.L., and Burkart, M.D. (2014). Fluorescent techniques for discovery and characterization of phosphopantetheinyl transferase inhibitors. The Journal of Antibiotics 67, 113-120.

Krömer, J.O., Dietmair, S., Jacob, S.S., and Nielsen, L.K. (2011). Quantification of I-alanylI-glutamine in mammalian cell culture broth: Evaluation of different detectors. Analytical Biochemistry 416, 129-131.

La Clair, J.J., Foley, T.L., Schegg, T.R., Regan, C.M., and Burkart, M.D. (2004). Manipulation of carrier proteins in antibiotic biosynthesis. Chemistry \& Biology 11, 195201.

Lamb, A.L. (2015). Breaking a pathogen's iron will: Inhibiting siderophore production as an antimicrobial strategy. Biochimica et Biophysica Acta (BBA) - Proteins and Proteomics $1854,1054-1070$. 
Lambalot, R.H., Gehring, A.M., Flugel, R.S., Zuber, P., LaCelle, M., Marahiel, M.A., Reid, R., Khosla, C., and Walsh, C.T. (1996). A new enzyme superfamily - the phosphopantetheinyl transferases. Chemistry \& Biology 3, 923-936.

Leblanc, C., Prudhomme, T., Tabouret, G., Ray, A., Burbaud, S., Cabantous, S., Mourey, L., Guilhot, C., and Chalut, C. (2012). 4'-Phosphopantetheinyl transferase PptT, a new drug target required for Mycobacterium tuberculosis growth and persistence in vivo. PLOS Pathogens 8, e1003097.

Lee, B., Diaz, G.A., Rhead, W., Lichter-Konecki, U., Feigenbaum, A., Berry, S.A., Le Mons, C., Bartley, J., Longo, N., Nagamani, S.C., et al. (2016). Glutamine and hyper-ammonemic crises in patients with urea cycle disorders. Molecular Genetics and Metabolism 117, $27-$ 32.

Lee, T.V., Johnson, L.J., Johnson, R.D., Koulman, A., Lane, G.A., Lott, J.S., and Arcus, V.L. (2010). Structure of a eukaryotic non-ribosomal peptide synthetase adenylation domain that activates a large hydroxamate amino acid in siderophore biosynthesis. The Journal of Biological Chemistry 285, 2415-2427.

Lewandowicz, A.M., Vepsäläinen, J., and Laitinen, J.T. (2006). The 'allosteric modulator' SCH-202676 disrupts $\mathrm{G}$ protein-coupled receptor function via sulphydryl-sensitive mechanisms. British Journal of Pharmacology 147, 422-429.

Li, P., Li, J., Guo, Z., Tang, W., Han, J., Meng, X., Hao, T., Zhu, Y., Zhang, L., and Chen, Y. (2015). An efficient blue-white screening based gene inactivation system for Streptomyces. Applied Microbiology Biotechnology 99, 1923-1933.

Liu, F., Zhang, J., Chen, R., Chen, L., and Deng, L. (2011). Highly effective colorimetric and visual detection of ATP by a DNAzyme-aptamer sensor. Chemistry \& Biodiversity 8, 311316.

Lott, J.S., and Lee, T.V. (2017). Revealing the inter-module Interactions of multi-modular Non-ribosomal Peptide Synthetases. Structure 25, 693-695.

Lund, P. (1986). L-glutamine and L-glutamate: UV-method with glutaminase and glutamate dehydrogenase. Methods of Enzymatic Analysis 8, 357-363. 
Lundin, A. (2000). Use of firefly luciferase in ATP-related assays of biomass, enzymes, and metabolites. Methods in Enzymology 305, 346-370.

Lundin, A., Rickardsson, A., and Thore, A. (1976). Continuous monitoring of ATPconverting reactions by purified firefly luciferase. Analytical Biochemistry 75, 611-620.

Luo, L., Kohli, R.M., Onishi, M., Linne, U., Marahiel, M.A., and Walsh, C.T. (2002). Timing of epimerization and condensation reactions in nonribosomal peptide assembly lines: kinetic analysis of phenylalanine activating elongation modules of tyrocidine synthetase B. Biochemistry 41, 9184-9196.

Marahiel, M.A. (2016). A structural model for multi-modular NRPS assembly lines. Natural Product Reports 33, 136-140.

Marahiel, M.A., Stachelhaus, T., and Mootz, H.D. (1997). Modular peptide synthetases involved in non-ribosomal peptide synthesis. Chemical Reviews 97, 2651-2674.

Márquez-Fernández, O., Trigos, Á., Ramos-Balderas, J.L., Viniegra-González, G., Deising, H.B., and Aguirre, J. (2007). Phosphopantetheinyl transferase CfwA/NpgA is required for Aspergillus nidulans secondary metabolism and asexual development. Eukaryotic Cell 6, 710-720.

McKenzie, D.C. (1999). Markers of excessive exercise. Canadian Journal of Applied Physiology 24, 66-73.

McQuade, T.J., Shallop, A.D., Sheoran, A., Delproposto, J.E., Tsodikov, O.V., and Garneau-Tsodikova, S. (2009). A nonradioactive high-throughput assay for screening and characterization of adenylation domains for non-ribosomal peptide combinatorial biosynthesis. Analytical Biochemistry 386, 244-250.

Mercer, A.C., and Burkart, M.D. (2007). The ubiquitous carrier protein-a window to metabolite biosynthesis. Natural Product Reports 24, 750-773.

Mizuhara, T., Oishi, S., Fujii, N., and Ohno, H. (2010). Efficient synthesis of pyrimido[1,2c] $[1,3]$ benzothiazin-6-imines and related tricyclic heterocycles by S(N)Ar-type C-S, C-N, or C-O bond formation with heterocumulenes. The Journal of Organic Chemistry 75, 265268. 
Mofid, M.R., Finking, R., and Marahiel, M.A. (2002). Recognition of hybrid peptidyl carrier proteins/acyl carrier proteins in Non-ribosomal peptide synthetase modules by the 4'-phophopantetheinyl transferases AcpS and Sfp. Journal of Biological Chemistry $277,17023-17031$.

Moore, J.C., Jin, H.-M., Kuchner, O., and Arnold, F.H. (1997). Strategies for the in vitro evolution of protein function: enzyme evolution by random recombination of improved sequences Edited by J. Wells. Journal of Molecular Biology 272, 336-347.

Mootz, H.D., and Marahiel, M.A. (1997). The tyrocidine biosynthesis operon of Bacillus brevis: complete nucleotide sequence and biochemical characterization of functional internal adenylation domains. Journal of Bacteriology 179, 6843-6850.

Mootz, H.D., Schörgendorfer, K., and Marahiel, M.A. (2002). Functional characterization of 4'-phosphopantetheinyl transferase genes of bacterial and fungal origin by complementation of Saccharomyces cerevisiae lys5. FEMS Microbiology Letters 213, 5157.

Müller, M., Ausländer, S., Ausländer, D., Kemmer, C., and Fussenegger, M. (2012). A novel reporter system for bacterial and mammalian cells based on the non-ribosomal peptide indigoidine. Metabolic Engineering 14, 325-335.

Myers, J.A., Curtis, B.S., and Curtis, W.R. (2013). Improving accuracy of cell and chromophore concentration measurements using optical density. BMC Biophysics 6, 4. Newman, D.J., and Cragg, G.M. (2016). Natural products as sources of new drugs from 1981 to 2014. Journal of Natural Products 79, 629-661.

Neyrolles, O., and Guilhot, C. (2011). Recent advances in deciphering the contribution of Mycobacterium tuberculosis lipids to pathogenesis. Tuberculosis 91, 187-195.

Novakova, R., Odnogova, Z., Kutas, P., Feckova, L., and Kormanec, J. (2010). Identification and characterization of an indigoidine-like gene for a blue pigment biosynthesis in Streptomyces aureofaciens CCM 3239. Folia Microbiologica 55, 119-125. 
Oganesyan, N., Ankoudinova, I., Kim, S.H., and Kim, R. (2007). Effect of osmotic stress and heat shock in recombinant protein overexpression and crystallization. Protein Expression and Purification 52, 280-285.

Okanojo, M., Miyashita, N., Tazaki, A., Tada, H., Hamazoto, F., Hisamatsu, M., and Noda, H. (2017). Attomol-level ATP bioluminometer for detecting single bacterium. Luminescence 32, 751-756.

Otten, L.G., Schaffer, M.L., Villiers, B.R., Stachelhaus, T., and Hollfelder, F. (2007). An optimized ATP/PP(i)-exchange assay in 96-well format for screening of adenylation domains for applications in combinatorial biosynthesis. Biotechnology Journal 2, 232240.

Owen, J.G. (2010). Characterisation, manipulation and directed evolution of nonribosomal peptide synthetase enzymes. (Doctoral dissertation) Victoria University of Wellington School of Biological Sciences.

Owen, J. G., Calcott, M. J., Robins, K.e J., and Ackerley, D. F. (2016). Generating Functional recombinant NRPS enzymes in the laboratory setting via peptidyl carrier protein engineering. Cell Chemical Biology 23, 1395-1406.

Owen, J.G., Copp, J.N., and Ackerley, D.F. (2011). Rapid and flexible biochemical assays for evaluating 4'-phosphopantetheinyl transferase activity. The Biochemical Journal 436, 709-717.

Owen, J.G., Robins, K.J., Parachin, N.S., and Ackerley, D.F. (2012). A functional screen for recovery of 4'-phosphopantetheinyl transferase and associated natural product biosynthesis genes from metagenome libraries. Environmental Microbiology 14, 11981209.

Pages, J.M., Masi, M., and Barbe, J. (2005). Inhibitors of efflux pumps in Gram-negative bacteria. Trends in Molecular Medicine 11, 382-389.

Parris, K.D., Lin, L., Tam, A., Mathew, R., Hixon, J., Stahl, M., Fritz, C.C., Seehra, J., and Somers, W.S. (2000). Crystal structures of substrate binding to Bacillus subtilis holo-(acyl carrier protein) synthase reveal a novel trimeric arrangement of molecules resulting in three active sites. Structure 8, 883-895. 
Patel, H.M., and Walsh, C.T. (2001). In vitro reconstitution of the Pseudomonas aeruginosa non-ribosomal peptide synthesis of pyochelin: characterization of backbone tailoring thiazoline reductase and N-methyltransferase activities. Biochemistry 40, 90239031.

Payne, J.A.E., Schoppet, M., Hansen, M.H., and Cryle, M.J. (2017). Diversity of nature's assembly lines - recent discoveries in non-ribosomal peptide synthesis. Molecular BioSystems 13, 9-22.

Petty, R.D., Sutherland, L.A., Hunter, E.M., and Cree, I.A. (1995). Comparison of MTT and ATP-based assays for the measurement of viable cell number. Journal of Bioluminescence and Chemiluminescence 10, 29-34.

Pierce, J.W., Schoenleber, R., Jesmok, G., Best, J., Moore, S.A., Collins, T., and Gerritsen, M.E. (1997). novel inhibitors of cytokine-induced IкB $\alpha$ phosphorylation and endothelial cell adhesion molecule expression show anti-inflammatory effects in Vivo. Journal of Biological Chemistry 272, 21096-21103.

Poole, K. (2001). Multidrug efflux pumps and antimicrobial resistance in Pseudomonas aeruginosa and related organisms. Journal of Molecular Microbiology and Biotechnology 3, 255-264.

Preuss, H.G., Bise, B.B., and Schreiner, G.E. (1966). The determination of glutamine in plasma and urine. Clinical Chemistry 12, 329-337.

Quadri, L.E.N., Sello, J., Keating, T.A., Weinreb, P.H., and Walsh, C.T. (1998). Identification of a Mycobacterium tuberculosis gene cluster encoding the biosynthetic enzymes for assembly of the virulence-conferring siderophore mycobactin. Chemistry \& Biology 5, 631-645.

Reimer, J.M., Aloise, M.N., Harrison, P.M., and Schmeing, T.M. (2016). Synthetic cycle of the initiation module of a formylating nonribosomal peptide synthetase. Nature 529, 239-242.

Reuter, K., Mofid, M.R., Marahiel, M.A., and Ficner, R. (1999). Crystal structure of the surfactin synthetase-activating enzyme Sfp: a prototype of the 4'-phosphopantetheinyl transferase superfamily. The Embo Journal 18, 6823-6831. 
Reverchon, S., Rouanet, C., Expert, D., and Nasser, W. (2002). Characterization of indigoidine biosynthetic genes in Erwinia chrysanthemi and role of this blue pigment in pathogenicity. Journal of Bacteriology 184, 654-665.

Rothmann, M., Kosa, N.M., and Burkart, M.D. (2014). Resin supported acyl carrier protein labeling strategies. RSC Advances 4, 9092-9097.

Rottier, K., Faille, A., Prudhomme, T., Leblanc, C., Chalut, C., Cabantous, S., Guilhot, C., Mourey, L., and Pedelacq, J.-D. (2013). Detection of soluble co-factor dependent protein expression in vivo: Application to the 4'-phosphopantetheinyl transferase PptT from Mycobacterium tuberculosis. Journal of Structural Biology 183, 320-328.

Röttig, M., Medema, M.H., Blin, K., Weber, T., Rausch, C., and Kohlbacher, O. (2011). NRPSpredictor2-a web server for predicting NRPS adenylation domain specificity. Nucleic Acids Research 39, W362-W367.

Samel, S.A., Czodrowski, P., and Essen, L.O. (2014). Structure of the epimerization domain of tyrocidine synthetase A. Acta Crystallographica Section D, Biological Crystallography 70, 1442-1452.

Samel, S.A., Wagner, B., Marahiel, M.A., and Essen, L.-O. (2006). The thioesterase domain of the fengycin biosynthesis cluster: A structural base for the macrocyclization of a non-ribosomal lipopeptide. Journal of Molecular Biology 359, 876-889.

Sancenón, F., Descalzo, A.B., Martínez-Máñez, R., Miranda, M.A., and Soto, J. (2001). A Colorimetric ATP Sensor Based on 1,3,5-Triarylpent-2-en-1,5-diones. Angewandte Chemie International Edition 40, 2640-2643.

Sansom, C. (2001). LPS inhibitors: key to overcoming multidrug-resistant bacteria? Drug Discovery Today 6, 499-500.

Sassetti, C.M., Boyd, D.H., and Rubin, E.J. (2003). Genes required for Mycobacterial growth defined by high density mutagenesis. Molecular Microbiology 48, 77-84.

Sissons, J.A. (2017). Engineering and characterisation of bacterial phosphopantetheinyl transferases and their peptide substrates. (Master's thesis) Victoria University of Wellington, School of Biological Sciences. 
Schalk, I.J., and Guillon, L. (2013). Pyoverdine biosynthesis and secretion in Pseudomonas aeruginosa: implications for metal homeostasis. Environmental Microbiology 15, 1661-1673.

Schauwecker, F., Pfennig, F., Grammel, N., and Keller, U. (2000). Construction and in vitro analysis of a new bi-modular polypeptide synthetase for synthesis of $\mathrm{N}$-methylated acyl peptides. Chemistry \& Biology 7, 287-297.

Schwarzer, D., Finking, R., and Marahiel, M.A. (2003). Non-ribosomal peptides: from genes to products. Natural Product Reports 20, 275-287.

Serrano, M., Ormazábal, A., Vilaseca, M.A., Lambruschini, N., Garcia-Romero, R., Meavilla, S., Perez-Dueñas, B., Pineda, M., Garcia-Cazorla, A., Campistol, J., et al. (2011). Assessment of plasma ammonia and glutamine concentrations in urea cycle disorders. Clinical Biochemistry 44, 742-744.

Simon, C., and Daniel, R. (2011). Metagenomic Analyses: Past and future trends. Applied and Environmental Microbiology 77, 1153-1161.

Sritharan, M. (2016). Iron homeostasis in Mycobacterium tuberculosis: mechanistic insights into siderophore-mediated iron uptake. Journal of Bacteriology 198, 2399-2409. Stachelhaus, T., Mootz, H.D., Bergendahl, V., and Marahiel, M.A. (1998). Peptide bond formation in nonribosomal peptide biosynthesis. Catalytic role of the condensation domain. The Journal of Biological Chemistry 273, 22773-22781.

Stachelhaus, T., Mootz, H.D., and Marahiel, M.A. (1999). The specificity-conferring code of adenylation domains in nonribosomal peptide synthetases. Chemistry \& Biology 6 , 493-505.

Starr, M.P., Cosens, G., and Knackmuss, H.-J. (1966). Formation of the blue pigment indigoidine by Phytopathogenic erwinia. Applied Microbiology 14, 870-872.

Stewart, E.J. (2012). Growing Unculturable Bacteria. Journal of Bacteriology 194, 41514160. 
Sunbul M., Marshall, N.j., Zou Y., Zhang, K., Yin, J. (2009) Catalytic Turnover-Based Phage Selection for Engineering the Substrate Specificity of Sfp Phosphopantetheinyl Transferase Journal of Molecular Biology 387

Süssmuth, R.D., and Mainz, A. (2017). Non-ribosomal peptide synthesis-principles and prospects. Angewandte Chemie International Edition 56, 3770-3821.

Takahashi, H., Kumagai, T., Kitani, K., Mori, M., Matoba, Y., and Sugiyama, M. (2007). Cloning and characterization of a Streptomyces single module type Non-ribosomal peptide synthetase catalyzing a blue pigment synthesis. Journal of Biological Chemistry 282, 9073-9081.

Takayama, K., Wang, C., and Besra, G.S. (2005). Pathway to synthesis and processing of mycolic acids in Mycobacterium tuberculosis. Clinical Microbiology Reviews 18, 81-101. Tan, B., Qiu, Y., Zou, X., Chen, T., Xie, G., Cheng, Y., Dong, T., Zhao, L., Feng, B., Hu, X., et al. (2013). Metabonomics identifies serum metabolite markers of colorectal cancer. Journal of Proteome Research 12, 3000-3009.

Tao, S. (2015). Colorimetric assay for I-glutamine and related assay kit (Google Patents). Tarry, M.J., Haque, A.S., Bui, K.H., and Schmeing, T.M. (2017). X-Ray crystallography and electron microscopy of cross- and multi-module non-ribosomal peptide synthetase proteins reveal a flexible architecture. Structure 25, 783-793.e784.

Taylor, A.L., Kudlow, B.A., Marrs, K.L., Gruenert, D.C., Guggino, W.B., and Schwiebert, E.M. (1998). Bioluminescence detection of ATP release mechanisms in epithelia. American Journal of Physiology - Cell Physiology 275, C1391-C1406.

Trinh, M.U., Blake, J., Harrison, J.R., Gerace, R., Ranieri, E., Fletcher, J.M., and Johnson, D.W. (2003). Quantification of glutamine in dried blood spots and plasma by tandem mass spectrometry for the biochemical diagnosis and monitoring of ornithine transcarbamylase deficiency. Clinical Chemistry 49, 681-684.

Tritsch, G.L., and Moore, G.E. (1962). Spontaneous decomposition of glutamine in cell culture media. Experimental Cell Research 28, 360-364. 
Tsuruoka, M., Hara, J., Hirayama, A., Sugimoto, M., Soga, T., Shankle, W.R., and Tomita, M. (2013). Capillary electrophoresis-mass spectrometry-based metabolome analysis of serum and saliva from neurodegenerative dementia patients. Electrophoresis 34, 28652872.

Uplekar, M., Weil, D., Lonnroth, K., Jaramillo, E., Lienhardt, C., Dias, H.M., Falzon, D., Floyd, K., Gargioni, G., Getahun, H., et al. WHO's new end TB strategy. The Lancet 385, 1799-1801.

Vickery, C.R., Kosa, N.M., Casavant, E.P., Duan, S., Noel, J.P., and Burkart, M.D. (2014). Structure, biochemistry, and inhibition of essential 4'-phosphopantetheinyl transferases from two species of Mycobacteria. ACS Chemical Biology 9, 1939-1944.

Villiers, B.R., and Hollfelder, F. (2009). Mapping the limits of substrate specificity of the adenylation domain of TycA. Chembiochem: A European Journal of Chemical Biology 10, 671-682.

Vriezen, N., Romein, B., Luyben, K.C., and van Dijken, J.P. (1997). Effects of glutamine supply on growth and metabolism of mammalian cells in chemostat culture. Biotechnology and Bioengineering 54, 272-286.

Walsh, C.T., Chen, H., Keating, T.A., Hubbard, B.K., Losey, H.C., Luo, L., Marshall, C.G., Miller, D.A., and Patel, H.M. (2001). Tailoring enzymes that modify non ribosomal peptides during and after chain elongation on NRPS assembly lines. Current Opinion in Chemical Biology 5, 525-534.

Walsh, C. T., O'Brien, R. V. and Khosla, C. (2013), Nonproteinogenic Amino Acid Building Blocks for Nonribosomal Peptide and Hybrid Polyketide Scaffolds. Angew. Chem. Int. Ed., 52: $7098-7124$.

Wang, H., Sivonen, K., and Fewer, D.P. (2015). Genomic insights into the distribution, genetic diversity and evolution of polyketide synthases and nonribosomal peptide synthetases. Current Opinion in Genetics \& Development 35, 79-85.

Weber, G., Schörgendorfer, K., Schneider-Scherzer, E., and Leitner, E. (1994). The peptide synthetase catalyzing cyclosporine production in Tolypocladium niveum is encoded by a giant 45.8-kilobase open reading frame. Current Genetics 26, 120-125. 
Weber, T., Baumgartner, R., Renner, C., Marahiel, M.A., and Holak, T.A. (2000). Solution structure of PCP, a prototype for the peptidyl carrier domains of modular peptide synthetases. Structure 8, 407-418.

Whitman, C.P. (2002). The 4-oxalocrotonate tautomerase family of enzymes: how nature makes new enzymes using a $\beta-\alpha-\beta$ structural motif. Archives of Biochemistry and Biophysics 402, 1-13.

Williams, E.M., Copp, J.N., and Ackerley, D.F. (2014). Site-saturation mutagenesis by overlap extension PCR. Methods in Molecular Biology (Clifton, NJ) 1179, 83-101.

Wise, D.R., and Thompson, C.B. (2010). Glutamine addiction: a new therapeutic target in cancer. Trends in Biochemical Sciences 35, 427-433.

Wong, H.C., Liu, G., Zhang, Y.M., Rock, C.O., and Zheng, J. (2002). The solution structure of acyl carrier protein from Mycobacterium tuberculosis. The Journal of Biological Chemistry 277, 15874-15880.

Xie, Z., Zhang, Z., Cao, Z., Chen, M., Li, P., Liu, W., Qin, H., Zhao, X., Tao, Y., and Chen, Y. (2017). An external substrate-free blue/white screening system in Escherichia coli. Applied Microbiology and Biotechnology 101, 3811-3820.

Xu, F., Gage, D., and Zhan, J. (2015). Efficient production of indigoidine in Escherichia coli. Journal of Industrial Microbiology \& Biotechnology 42, 1149-1155.

Yang, H., lerapetritou, M.G., and Roth, C.M. (2010). Effects of amino acid transport limitations on cultured hepatocytes. Biophys Chem 152, 89-98.

Yasgar, A., Foley, T., Jadhav, A., Inglese, J., Burkart, M., and Simeonov, A. (2010). A strategy to discover inhibitors of Bacillus subtilis surfactin-type phosphopantetheinyl transferase. Molecular BioSystems 6, 365-375.

Yin, J., Straight, P.D., Hrvatin, S., Dorrestein, P.C., Bumpus, S.B., Jao, C., Kelleher, Neil L., Kolter, R., and Walsh, C.T. (2007). Genome-wide high-throughput mining of naturalproduct biosynthetic gene clusters by phage display. Chemistry \& Biology 14, 303-312. Yin, J., Straight, P.D., McLoughlin, S.M., Zhou, Z., Lin, A.J., Golan, D.E., Kelleher, N.L., Kolter, R., and Walsh, C.T. (2005). Genetically encoded short peptide tag for versatile 
protein labeling by Sfp phosphopantetheinyl transferase. Proceedings of the National Academy of Sciences of the United States of America 102, 15815-15820.

Yu, D., Xu, F., Valiente, J., Wang, S., and Zhan, J. (2013). An indigoidine biosynthetic gene cluster from Streptomyces chromofuscus ATCC 49982 contains an unusual IndB homologue. Journal of Industrial Microbiology \& Biotechnology 40, 159-168.

Zandvoort, E., Baas, B.-J., Quax, W.J., and Poelarends, G.J. (2011). Systematic screening for catalytic promiscuity in 4-oxalocrotonate tautomerase: enamine formation and aldolase activity. Chembiochem : A European Journal of Chemical Biology 12, 602-609. Zandvoort, E., Geertsema, E.M., Quax, W.J., and Poelarends, G.J. (2012). Enhancement of the promiscuous aldolase and dehydration activities of 4-Oxalocrotonate tautomerase by protein engineering. Chembiochem : A European Journal of Chemical Biology 13, 1274-1277.

Zeng, X., Zhang, X., Yang, W., Jia, H., and Li, Y. (2012). Fluorescence detection of adenosine triphosphate through an aptamer-molecular beacon multiple probe. Analytical Biochemistry 424, 8-11.

Zhang, K., He, J., Yang, M., Yen, M., and Yin, J. (2009). Identifying natural product biosynthetic genes from a soil metagenome by using T7 phage selection. Chembiochem : A European Journal of Chemical Biology 10, 2599-2606.

Zhou, Z., Cironi, P., Lin, A.J., Xu, Y., Hrvatin, S., Golan, D.E., Silver, P.A., Walsh, C.T., and Yin, J. (2007). genetically encoded short peptide tags for orthogonal protein labeling by Sfp and AcpS phosphopantetheinyl transferases. ACS Chemical Biology 2, 337-346.

Zhou, Z., Koglin, A., Wang, Y., McMahon, A.P., and Walsh, C.T. (2008). An eight residue fragment of an Acyl Carrier Protein suffices for post-translational introduction of fluorescent Pantetheinyl Arms in protein modification in vitro and in vivo. Journal of the American Chemical Society 130, 9925-9930. 
Zimhony, O., Schwarz, A., Raitses-Gurevich, M., Peleg, Y., Dym, O., Albeck, S., Burstein, Y., and Shakked, Z. (2015). AcpM, the meromycolate extension acyl carrier protein of Mycobacterium tuberculosis, is activated by the $4^{\prime}$-phosphopantetheinyl transferase PptT, a potential target of the multistep mycolic acid biosynthesis. Biochemistry 54, 2360-2371. 